\title{
WestVirginiaUniversity
}

THE RESEARCH REPOSITORY @ WVU

Graduate Theses, Dissertations, and Problem Reports

2019

\section{Exploring the Diffuse Neutral Hydrogen in and Around Nearby Galaxies}

Amy Sardone

aesardone@gmail.com

Follow this and additional works at: https://researchrepository.wvu.edu/etd

Part of the External Galaxies Commons

\section{Recommended Citation}

Sardone, Amy, "Exploring the Diffuse Neutral Hydrogen in and Around Nearby Galaxies" (2019). Graduate Theses, Dissertations, and Problem Reports. 7484.

https://researchrepository.wvu.edu/etd/7484

This Dissertation is protected by copyright and/or related rights. It has been brought to you by the The Research Repository @ WVU with permission from the rights-holder(s). You are free to use this Dissertation in any way that is permitted by the copyright and related rights legislation that applies to your use. For other uses you must obtain permission from the rights-holder(s) directly, unless additional rights are indicated by a Creative Commons license in the record and/ or on the work itself. This Dissertation has been accepted for inclusion in WVU Graduate Theses, Dissertations, and Problem Reports collection by an authorized administrator of The Research Repository @ WVU.

For more information, please contact researchrepository@mail.wvu.edu. 


\title{
Exploring the Diffuse Neutral Hydrogen in and Around Nearby Galaxies
}

\author{
Amy Sardone \\ Dissertation submitted \\ to the Eberly College of Arts and Sciences \\ at West Virginia University \\ in partial fulfillment of the requirements for the degree of \\ Doctor of Philosophy \\ in \\ Physics \\ D.J. Pisano, Ph.D., Chair \\ Sarah Burke-Spolaor, Ph.D. \\ Claude Carignan, Ph.D. \\ Maura McLaughlin, Ph.D. \\ Department of Physics and Astronomy \\ Morgantown, West Virginia, USA \\ 2019
}

Keywords: Physics, Astronomy, Galaxies, HI, CGM

Copyright 2019 Amy Sardone 
Abstract

\title{
Exploring the Diffuse Neutral Hydrogen in and Around Nearby Galaxies
}

\author{
Amy Sardone
}

We explore the environment of 36 nearby galaxies in neutral hydrogen (H I) as part of the MeerKAT H I Observations of Nearby Galactic Objects; Observing Southern Emitters (MHONGOOSE) survey with the Robert C. Byrd Green Bank Telescope (GBT), and the Imaging Galaxies Intergalactic and Nearby Environment (IMAGINE) survey with the Parkes Radio Telescope. We obtained deep observations of each of these galaxies, reaching column density detection sensitivities as low as $N_{\mathrm{HI}} \sim 10^{17} \mathrm{~cm}^{-2}$, which allowed us to quantify the amount of diffuse HI in both samples of galaxies. This allows us to search for evidence of gas that could be accreting onto galaxies via filamentary structures from the cosmic web. These structures are predicted to be low column density gas flows, which should be seen within the dark matter halo of a galaxy.

With our sample of 18 MHONGOOSE galaxies, we are able to make comparisons to environmental factors that are associated with cold accretion. Our GBT observations of diffuse H I in these galaxies do reveal trends in properties associated with this accretion, such as higher amounts of diffuse H I in galaxies with baryonic masses below a threshold of $10^{10.3} M_{\odot}$. Our data also revealed trends when looking at the dark matter halo masses and rotation velocities of these MHONGOOSE galaxies.

We take a deep look into one of the IMAGINE galaxies, ESO 214-17, revealing a new low column density $\mathrm{H}$ I bridge connecting the central galaxy to a companion. We also present a look at the first data sample from the IMAGINE survey with the Parkes telescope. These 18 galaxies were mapped to extremely low H I column densities $\left(N_{\mathrm{HI}} \sim 10^{17} \mathrm{~cm}^{-2}\right)$, and extend out to or beyond the virial radius of each galaxy. Encompassing the entire dark matter halo allows us to quantify the amount of HI throughout the halo, and subsequently the circumgalactic medium of each galaxy. Our environmental comparisons with properties associated with cold accretion do not reveal strong trends in the IMAGINE sample, however when combining all 36 galaxies discussed here we do see trends consistent with cold mode accretion. These trends are revealed when we look at baryonic masses, galaxy densities, and to a lesser degree with dark matter halo masses and rotation velocities. 


\section{Acknowledgments}

I would like to express my deepest gratitude to my husband, John. Your support has carried me through graduate school and through this process of writing a dissertation. You not only encouraged me to continue when I wanted to quit, but you gave me everything that you could have to make this process as easy as possible. Thank you for taking wonderful full-time care of our kids while I wrote, for your many pep-talks, for being a great listener, and for cheering me on every step of the way. I could not be more sincere when I say that I could not have done this without you. I love you with all of my heart.

I would like to thank my kids, Stella and Henry, for lighting up every day. Henry, one of my favorite parts of the day is seeing your wide smile when you get home from school and you run to give me a hug. Stella, you are the smartest 7-year-old I know, and I love watching you learn and teaching Henry all about galaxies. I'm doing this for you, sweet girl.

To my advisor, D.J. Pisano, thank you for taking me on as a graduate student when I had little experience. Your faith in me was invaluable. Thank you for sending me literally all over the world. I love to travel, and I am so grateful to have been able to attend conferences and to train on telescopes around the world. I have felt lucky to have you for an advisor. I've yet to ask a question you couldn't answer, which somehow still astonishes me, even after four years. You have been kind, flexible, easygoing, and supportive. Your demand for equity and inclusivity in the department has never gone unnoticed.

I would also like to thank my undergraduate research advisor and committee member, Maura McLaughlin, for stopping me in the hall one day, getting to know me, and offering me a chance to do my first scientific research project. I had never seen anyone code as quickly as you, and I don't think I have since. Thank you for your patience when I was an extreme novice.

Thank you to all of my committee members, D.J., Maura, Sarah Burke-Spolaor, and Claude Carignan, for reading this dissertation and for providing feedback that not only improves the work, but helps me improve as a scientist.

Thank you to the best office mates, Lucas Hunt and Nick Pingel. You have both enriched my life and made being in the office fun, Nick - through our mutual love of WV beers, and Lucas - for always introducing me to great, new music. Thank you for the help you've both given me over the years.

I would also like to thank the astrophysicist whom I met while teaching his son voice lessons. Meeting you sparked a flame I didn't know existed.

Also, thank you to my favorite physics buddies, Nihan Pol and Josh Mascoop, for the fun, the study groups, the commiseration, and most of all for the deep friendship that I cherish. Go team ANJ! 


\section{Table of Contents}

List of Tables $\quad$ vi

List of Figures $\quad$ vii

1 Introduction 1

1.1 Why Have Galaxies Not Run Out of Gas? . . . . . . . . . . . . . . . 1

1.2 A Look At Neutral Hydrogen . . . . . . . . . . . . . . . . . . . 7

1.3 Thesis Outline . . . . . . . . . . . . . . . . 11

2 Evidence of Ongoing Interaction from IMAGINE Galaxy ESO 214-17 13

2.1 Abstract . . . . . . . . . . . . . . . 13

2.2 Introduction . . . . . . . . . . . . . . . . . . . . 14

2.3 Target . . . . . . . . . . . . . . . . . . . . . . . . . . . . . . . .

2.4 Observations and Reduction . . . . . . . . . . . . . . . 22

2.4 .1 Observations . . . . . . . . . . . . . 22

2.4 Data Reduction . . . . . . . . . . . . . . . . 23

2.5 Results ............................. 26

2.5.1 Spectra ...................... 26

2.5.2 Galaxy Properties . . . . . . . . . . . . . . . 26

2.5.3 Other Hi sources in the cube . . . . . . . . . . . . . . . . . . 35

2.6 Discussion . . . . . . . . . . . . . . . . . . . . . . . 37

2.7 Conclusions and Future Work . . . . . . . . . . . . . . . . . . . . . 42

3 Analysis of the Diffuse H I Environment Around 18 MHONGOOSE Galaxies 45

3.1 Abstract . . . . . . . . . . . . . . . . 45

3.2 Introduction . . . . . . . . . . . . . . . . . 46

3.3 MHONGOOSE Galaxy Sample . . . . . . . . . . . . . . . . . . . 48

3.4 Observations and Data Reduction . . . . . . . . . . . . . . 49

3.5 Properties of galaxies . . . . . . . . . . . . . . . . 51

3.5.1 NGC $1744 \ldots \ldots \ldots \ldots$. . . . . . . . . . . . . . . . . . . . . . . . . . . . 51

3.5 .2 NGC $7424 \ldots \ldots \ldots \ldots$

3.5.3 Discussion of the Satellites around NGC 1744 and NGC 7424. 55

3.6 Analysis . . . . . . . . . . . . . . . . . 57

3.6.1 Total integrated H I flux profiles . . . . . . . . . . . . . 57

3.6.2 Derived galaxy properties . . . . . . . . . . . . 58

3.6 .3 GBT beam model . . . . . . . . . . . . . . . . . 60

3.6.4 Integrated intensity images (Moment 0) . . . . . . . . . . 62

3.6.5 Cumulative H I vs. $N_{\mathrm{HI}}$. . . . . . . . . . . . . . . . . . . . 63

3.6.6 Radial $N_{\mathrm{HI}}$..................... . . 65

3.6.7 Radial Flux . . . . . . . . . . . . . . . . . 66

3.7 Results . . . . . . . . . . . . . . . . . . . 67

3.7.1 Bin $1\left(6<\log _{10}\left(\mathrm{M}_{\mathrm{HI}}\right)<8 M_{\odot}\right) \ldots \ldots . \ldots . \ldots 68$

3.7.2 Bin $2\left(8<\log _{10}\left(\mathrm{M}_{\mathrm{HI}}\right)<8.5 M_{\odot}\right) \ldots \ldots . \ldots . \ldots 6$ 
3.7.3 Bin $3\left(8.5<\log _{10}\left(\mathrm{M}_{\mathrm{HI}}\right)<9 M_{\odot}\right) \ldots \ldots \ldots \ldots \ldots \ldots$

3.7.4 Bin $4\left(9<\log _{10}\left(\mathrm{M}_{\mathrm{HI}}\right)<9.5 M_{\odot}\right) \ldots \ldots \ldots \ldots \ldots$

3.7.5 Bin $5\left(9.5<\log _{10}\left(\mathrm{M}_{\mathrm{HI}}\right)<10 M_{\odot}\right) \ldots \ldots \ldots \ldots \ldots . \ldots . \ldots 71$

3.7.6 Bin $6\left(10<\log _{10}\left(\mathrm{M}_{\mathrm{HI}}\right)<11 M_{\odot}\right) \ldots \ldots \ldots \ldots \ldots \ldots$

3.8 Discussion . . . . . . . . . . . . . . . . . . . . . . . . . . . . . . . . . 73

3.8 .1 Extraplanar gas . . . . . . . . . . . . . . . . . . . . 73

3.8.2 Fraction of low $N_{\mathrm{HI}} \ldots \ldots \ldots \ldots \ldots \ldots \ldots \ldots$

3.8.3 Depletion timescales . . . . . . . . . . . . . . . . . 97

3.9 Summary . . . . . . . . . . . . . . . . . . . . . . . . . . . . . 98

4 Probing the Full Virial Volume of IMAGINE Galaxies 101

4.1 Abstract . . . . . . . . . . . . . . . . . . . . . . . . . . 101

4.2 Introduction . . . . . . . . . . . . . . . . . . . . . . 103

4.3 The IMAGINE Survey and Galaxy Sample . . . . . . . . . . . 104

4.4 Observations and Data Reduction . . . . . . . . . . . . . . . . . . 109

4.5 Analysis . . . . . . . . . . . . . . . . . . . . . . . . . . 113

4.5.1 H I Measurements and Galaxy Properties . . . . . . . . . . . 113

4.5 .2 Differences in Analysis . . . . . . . . . . . . . . . . 117

4.6 Results . . . . . . . . . . . . . . . . . . . . . . . . . . . . . . . . . . . 129

4.6.1 Results from radial and cumulative analysis . . . . . . . . 129

4.6 .2 Diffuse H i Fraction . . . . . . . . . . . . . . . . . . . . . . 149

4.7 Discussion . . . . . . . . . . . . . . . . . . . . . . . . . . . . . 154

4.7.1 How much H I mass is in the CGM? . . . . . . . . . . . 154

4.7.2 Is there enough CGM mass to sustain star formation? . . . . 156

4.8 Summary . . . . . . . . . . . . . . . . . . . . . 160

5 Conclusions 162

5.1 Main Conclusions . . . . . . . . . . . . . . . . . . . . . . . 162

5.2 Future Plans . . . . . . . . . . . . . . . . 166 


\section{List of Tables}

2.1 Properties of ESO 214-17/18 . . . . . . . . . . . . . . . 21

2.3 Observing parameters for IMAGINE galaxy ESO 214-17 . . . . . . . 22

2.4 Observing parameters for IMAGINE galaxy ESO 214-17 . . . . . . . 22

2.5 Properties of all detected galaxies in ESO 214-17 datacube . . . . . . 25

3.1 Basic Properties of Sample of MHONGOOSE Galaxies . . . . . . . . 60

3.2 H I Measurements and Derived Properties of MHONGOOSE Galaxies 61

3.3 Depletion timescales of the disk and halo . . . . . . . . . . . . 97

4.1 Basic Properties of Sample of IMAGINE Galaxies . . . . . . . . . . . 106

4.2 Comparison of derived stellar and halo masses . . . . . . . . . . . . . 107

4.3 H I Measurements and Derived Properties of IMAGINE Galaxies . . . 114

4.4 Depletion timescales of the disk and halo . . . . . . . . . . . . 158 


\section{List of Figures}

1.1 Gas flows in a disk galaxy $\ldots \ldots \ldots \ldots \ldots$

2.1 Optical image of ESO $214-17 \ldots \ldots \ldots \ldots$

2.2 Total integrated spectra of ESO 214-17 and ESO 214-18 $\ldots \ldots . .27$

2.3 Position-velocity diagram from ESO $214-17 \ldots \ldots \ldots \ldots$

2.4 ESO 214-17 channel map . . . . . . . . . . . . . . . . . . . . 30

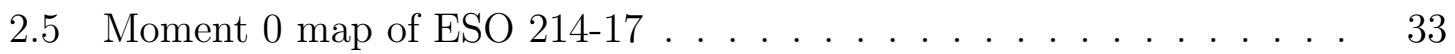

2.6 ESO 214-17 Moment 1 and 2 maps . . . . . . . . . . . . . . . 34

2.7 Spectra and Moment 0 map of ESO 214-15 . . . . . . . . . 35

2.8 Spectra of ESO $264-35 \ldots \ldots \ldots \ldots \ldots \ldots$

3.1 Histogram of number of sources in each H I mass bin $\ldots \ldots \ldots$

3.2 Moment map of NGC $1744 \ldots \ldots \ldots \ldots \ldots \ldots$

3.3 Moment map of NGC $7424 \ldots \ldots \ldots \ldots \ldots \ldots$

3.4 Spectra of NGC1744 and NGC7424 . . . . . . . . . . . 54

3.5 Other detections in NGC1744 and NGC7424 maps . . . . . . . . . 56

3.6 ESO300-G016 . . . . . . . . . . . . . . . . . . . . . . 74

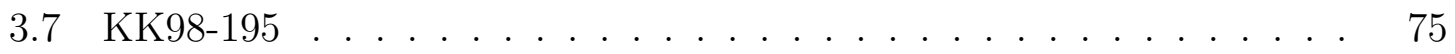

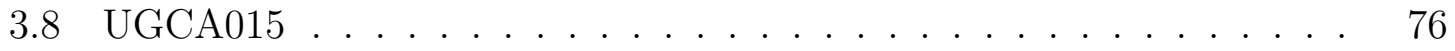

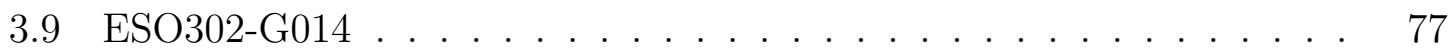

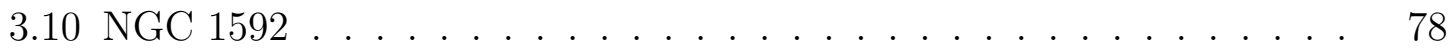

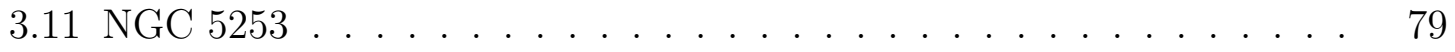

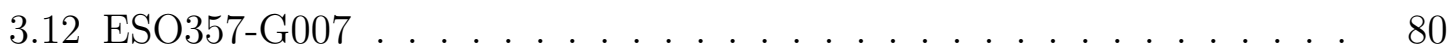

3.13 KKS2000-23 . . . . . . . . . . . . . . . . . . . . . . 81

3.14 UGCA307 . . . . . . . . . . . . . . . . . . . . . . . . . . 82

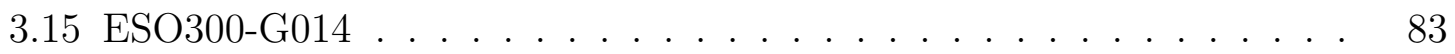

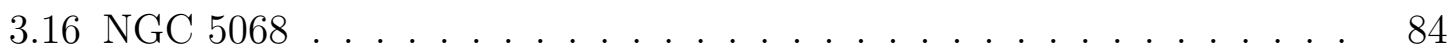

3.17 UGCA320 . . . . . . . . . . . . . . . . . . . . . . . 85

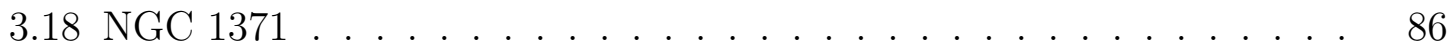

3.19 NGC $1744 \ldots \ldots \ldots \ldots \ldots$

3.20 NGC $3511 \ldots \ldots \ldots \ldots \ldots$

3.21 NGC $5170 \ldots \ldots \ldots \ldots \ldots$

3.22 NGC $7424 \ldots \ldots \ldots \ldots \ldots \ldots$

3.23 UGCA250 . . . . . . . . . . . . . . . . . . . . . . . . 91

$3.24 f_{19}$ vs. environmental properties . . . . . . . . . . . . 93

4.1 Histogram of Parkes-IMAGINE galaxy properties . . . . . . . . 110

4.2 Moment map of ESO138-10 . . . . . . . . . . . . . . . . 118

4.3 Moment maps of ESO138-10 and ESO214-17 . . . . . . . . . . 119

4.4 Moment maps of IC5201 and NGC625 . . . . . . . . . . . . 120

4.5 Moment maps of NGC1433 and NGC1744 . . . . . . . . . . . 121

4.6 Moment maps of NGC1792 and NGC2188 . . . . . . . . . . . 122

4.7 Moment maps of NGC2835 and NGC3137 . . . . . . . . . . 123 
4.8 Moment maps of NGC3175 and NGC7424 . . . . . . . . . . . . . . . 124

4.9 Moment maps of NGC7793 . . . . . . . . . . . . . . . . . . 125

4.10 Moment maps of ESO209-9 and NGC24 . . . . . . . . . . . . . . 126

4.11 Moment maps of NGC45 and NGC1512 . . . . . . . . . . . . . 127

4.12 ESO138-10 . . . . . . . . . . . . . . . . . 130

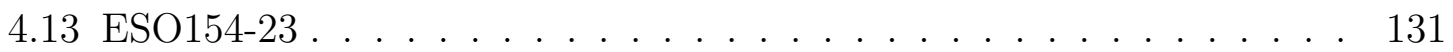

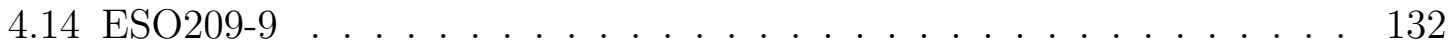

4.15 ESO214-17. . . . . . . . . . . . . . . . . . 133

4.16 IC5201 . . . . . . . . . . . . . . . . . . . . . . . 134

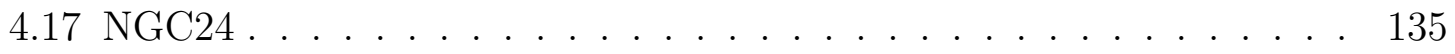

4.18 NGC45 . . . . . . . . . . . . . . . . . . . 136

4.19 NGC625 . . . . . . . . . . . . . . . . . . . . . 137

$4.20 \mathrm{NGC1433} \ldots \ldots \ldots \ldots \ldots$

$4.21 \mathrm{NGC1512} \ldots \ldots \ldots \ldots \ldots \ldots$

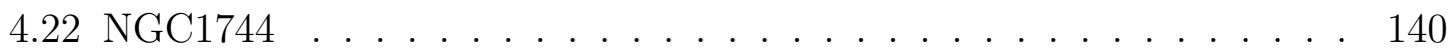

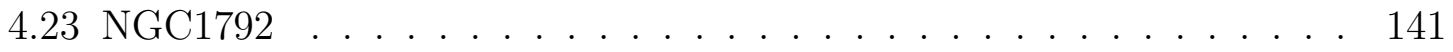

4.24 NGC2188 . . . . . . . . . . . . . . . . . . . . . . . . . . 142

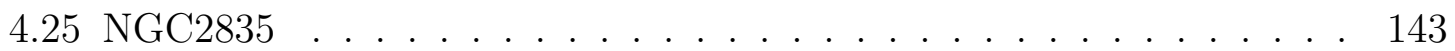

4.26 NGC3137 . . . . . . . . . . . . . . . . . . . . . . . . . . . . . . . . . . . . . . . . . . . . . . .

4.27 NGC3175 . . . . . . . . . . . . . . . . . . . . . 145

4.28 NGC7424 . . . . . . . . . . . . . . . . . . . . . . 146

4.29 NGC7793 . . . . . . . . . . . . . . . . . . . . . 147

$4.30 f_{19}$ vs. environmental properties . . . . . . . . . . . . . . 153

4.31 Halo mass histogram for IMAGINE galaxies . . . . . . . . . . . 155

$4.32 f_{C G M}$ vs. environmental properties . . . . . . . . . . . . 157

5.1 Sensitivities of all large HI surveys . . . . . . . . . . . . . . . 163

$5.2 f_{19}$ environmental analysis with all galaxies . . . . . . . . . 165 


\section{Chapter 1}

\section{Introduction}

The picture of how galaxies form and have evolved over time is still being established. Current models depict dark matter structures which harbor gas at their centers. In some of these dark matter structures, or halos, the gas cools and forms stars, eventually forming galaxies (e.g., Cole et al., 2000). These galaxies continue to form stars and grow larger. Over cosmic time, the rate at which these galaxies formed stars grew until it peaked around a redshift of $z \sim 2$ (e.g., Madau \& Dickinson, 2014), after which it has continued to decline through the present day. Many questions on galaxy formation and evolution remain unanswered. Some of these questions include: what is the mechanism through which gas cools and falls onto a galaxy? How is the gas and the dark matter distributed throughout a galaxy's dark matter halo? How are

galaxies connected to their halos? In this thesis we address these questions through observations of nearby galaxies and their dark matter halos.

\subsection{Why Have Galaxies Not Run Out of Gas?}

We must first set up the picture as we know it so far. Hierarchical galaxy formation models begin with a cosmological model. The model considered to be the current standard is $\Lambda \mathrm{CDM}$, a cold dark matter Universe with cosmological constants. Under these conditions, dark matter halos form and grow through mergers and ac- 
cretion (Peebles, 1982; Davis et al., 1985; White \& Frenk, 1991; Springel et al., 2005; Schaye et al., 2015). Gas inside a dark matter halo can cool and fall into the gravitational potential well to form a galaxy. We call the gas within the halo, excluding the galaxy disk, the circumgalactic medium (CGM). The size of the dark matter halo is determined by the galaxy's virial radius $R_{v i r}$, the radius at which the density is equal to 200 times the density of the Universe. This factor may vary, but 200 has been shown to approximately represent the density below which material has been virialized (White, 2001). Galaxy halos are connected to other galaxy halos via the intergalactic medium (IGM). This IGM is made up of filamentary structures, called the cosmic web (Bond et al., 1996), along which gas and galaxies lie. Now we can look at what the gas is doing once it reaches the halo. Much of this gas is diffuse and ionized, and is therefore difficult to detect (e.g., Joung et al., 2012). However, theories and models of galaxy formation use gas accretion, galaxy mergers, and feedback processes to explain what we see in galaxies today (e.g., Schechter, 1976; Tinsley, 1980; Navarro et al., 1996; Kereš et al., 2005; Leitner \& Kravtsov, 2011; Hirschmann et al., 2012). The cartoon in Figure 1.1 illustrates the current picture of gas flow in the CGM, and includes the main players in one of the key questions in galaxy evolution: how galaxies get their gas. A galaxy that is actively forming stars is, by default, using some kind of fuel to convert into those stars. This fuel is molecular hydrogen, or $H_{2}$. The question of where this fuel comes from, or how it is fed into the galaxy, is yet to be definitively answered. The rate at which galaxies convert their gas into stars can be estimated across redshifts, where the highest star formation rates per unit volume are seen between redshifts of $z \sim 2$ (Madau \& Dickinson, 2014), and have 


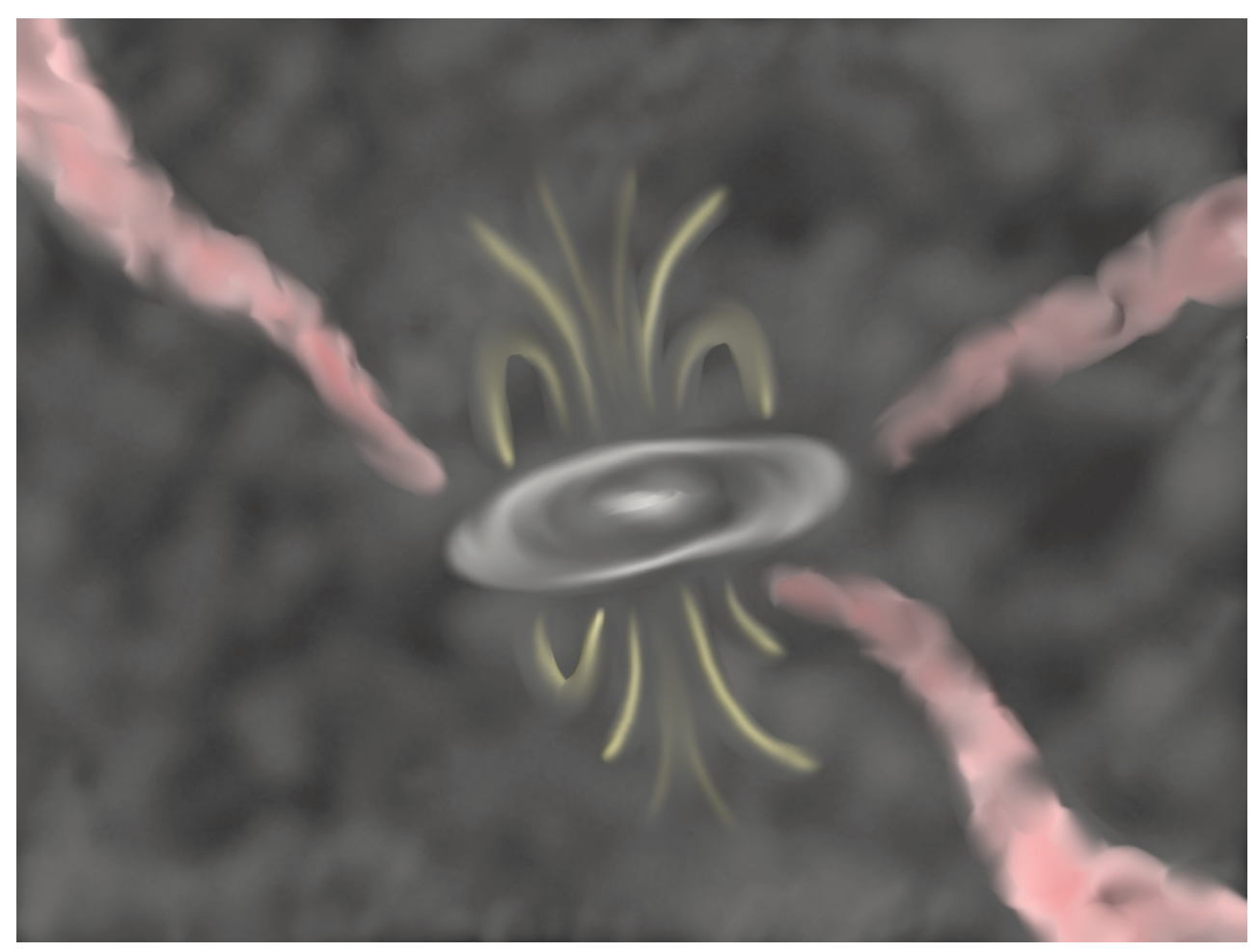

Figure 1.1: The CGM of a disk galaxy displaying filamentary accretion in pink, outflows and recycling of gas in yellow.

been decreasing ever since. These star formation rates can be used to calculate gas depletion timescales, which tell us how quickly the galaxy would run out of gas were it not replenished. Observations show us that galaxies across redshifts have small gas depletion timescales (Kennicutt, 1983) of less than a few Gyr (Leroy et al., 2008; Bigiel et al., 2008; Schiminovich et al., 2010; Genzel et al., 2010; Daddi et al., 2010; Bigiel et al., 2011; Kennicutt \& Evans, 2012; Tacconi et al., 2013; Leroy et al., 2013). The implication here is that if this were the whole picture, most galaxies would be red and dead, so to speak. However, we see enough blue, star-forming galaxies across redshifts to know this is not the case.

So what could be fueling this continued star formation? We know that some star formation occurs from recycled gas returning to the interstellar medium (ISM) of 
a galaxy. This gas can be identified by its metal-enrichment following the deaths of stars (Tumlinson et al., 2011; Werk et al., 2014; Rubin et al., 2014). Star formation can also be induced by gas accreting onto the disk of the galaxy from the IGM (Larson, 1972a,b). This gas would be pristine, or metal-poor, as it would not yet have interacted with the galaxy's stellar population. If a sufficient amount of this gas is accreting onto the disk, it could provide enough fuel to sustain star formation. As gas flows along the filaments in the IGM, it enters the dark matter halo of a galaxy, and follows one of two temperature paths: hot or cold. Simulations from Kereš et al. (2005) show that nearly half of the gas that enters the halo becomes shock heated to the virial temperature of the galaxy. For a Milky Way-type galaxy, this temperature is $T \sim 10^{6} \mathrm{~K}$. It then cools, condenses, and starts forming stars. This is called accretion in the "hot mode" which dominates in galaxies with total baryonic masses $M_{\text {bary }} \gtrsim 10^{10.3} M_{\odot}$ or with dark matter halos $M_{\text {halo }} \gtrsim 10^{11.4} M_{\odot}$. The gas in the hot mode has become almost completely ionized by this point, and the cooling timescales are very short. The other portion of the gas, still mostly ionized with a small percentage neutral, continues through the halo along filaments without ever heating to the virial temperature. This gas flows directly from the IGM via filamentary structures, through the galaxy's CGM before falling onto the disk, and stays below temperatures of $T<10^{5} \mathrm{~K}$. This "cold mode" accretion dominates in lower mass galaxies, and at low redshift is dependent on galaxy environment where low density environments are favored. Ideal parameters to observe this gas at low redshift then, would be to search galaxies with total baryonic masses $M_{\text {bary }} \lesssim 10^{10.3} M_{\odot}$, dark matter halos $M_{\text {halo }} \lesssim 10^{11.4} M_{\odot}$, and galaxy density $n_{\text {gal }} \lesssim 1 \mathrm{Mpc}^{-1}$. 
Gas accreting in this way is much more difficult to observe directly. In the local Universe, we can use the 21-cm H I emission line to trace the pristine gas accreting from the IGM. However, at redshifts greater than $z \sim 0$, we must use absorption lines where emission lines are too weak. Absorption lines provide indirect evidence for accretion through the halo from the IGM, and many have been detected at these higher redshifts, although detections are limited to sightlines associated with background quasars (QSOs). We can use the kinematics from absorption lines in the spectrum of a galaxy to determine if the source is flowing toward or away from the galaxy, by whether the line has been redshifted (inflow) or blueshifted (outflow) with respect to the rest frequency. This is currently the best evidence of gas accretion onto a galaxy, examples of which include a redshifted neutral sodium doublet at $0.11>z>0.54$ from Sato et al. (2009) and in 13 Seyfert galaxies from Krug et al. (2010). Lyman limit systems (LLSs), which are absorption detections of H I with column densities between $10^{17} \lesssim N_{H I}<10^{19} \mathrm{~cm}^{-2}$, also likely describe cold streams of gas in which inflow vs. outflow can be characterized by metallicity, where lower metallicity would presumably indicate inflows that have not yet been enriched by the galaxy (Lehner, 2017). Numerous detections of LLSs and their metallicities include Prochaska (1999); Lehner et al. (2013); Fox et al. (2013). Tumlinson et al. (2013) used the Cosmic Origins Spectrograph on the Hubble Space Telescope to probe the halos of 44 galaxies at $z=0.15-0.35$ using background QSOs. They detected absorption in the halos from HI associated with the galaxies at HI column densities above $10^{16} \mathrm{~cm}^{-2}$. Locally, we can use the emission line to give us kinematic information, and to directly detect extremely diffuse gas associated with a galaxy's CGM. In the nearby Universe, there 
are few examples of H I observations of gas accreting onto galaxies, but some of the strongest cases are M31 and NGC 891, among others. Braun \& Thilker (2004) and later Wolfe et al. $(2013,2016)$ revealed faint H I clouds between M31 and M33, a potential indication of accretion, from their extremely sensitive observations. NGC 891 has been repeatedly observed in H I, revealing a plume or filament of H I gas perpendicular to the plane, which is likely the result of either gas recycling from the disk or accretion from the CGM (e.g., Oosterloo et al., 2007; Pingel et al., 2018).

Fraternali \& Tomassetti (2012) analyzed 21 galaxies from the THINGS (Walter et al., 2008) survey, an H I survey using the Karl G. Jansky Very Large Array radio telescope, to derive gas accretion rates for these galaxies. They found that for most of these galaxies, the gas infall was very low and that the amount of gas already available was sufficient for sustaining star formation, eliminating the need for accretion. Popping et al. (2009) simulate the distribution of H I in a 30 square degree field at $z=0$ with $\mathrm{H}$ I column densities between $N_{\mathrm{HI}}=10^{14}$ and $10^{21} \mathrm{~cm}^{-2}$. Their simulations reveal diffuse substructure around galaxies with $N_{\mathrm{HI}}$ column densities as low as a few times $10^{16} \mathrm{~cm}^{-2}$. Modern radio telescopes, given enough integration time, should be able to reach column density sensitivities comparable to this. To understand why $\mathrm{HI}$ is such an important ingredient in understanding the formation and evolution of galaxies, we must look at what uniquely qualifies H I to characterize properties of galaxies. 


\subsection{A Look At Neutral Hydrogen}

When astronomers refer to $\mathrm{H}$, they are referring to neutral atomic hydrogen, or one electron orbiting one proton, making up the hydrogen atom. Because electrons and protons are Fermions, we know that they each have half-integer spins (Pauli, 1940). When a magnetic field causes these two Fermions to interact, the ground state of the atom is split into two separate states of different energy levels. This is known as the hyperfine splitting of the ground state, first observed by A. A. Michelson in 1881. When this occurs, the electron will be in either the upper energy state or the lower energy state. What defines the higher and lower energies of the atom is the total spin angular momentum, where the two states are set by the spins being parallel or antiparallel. When the spins are aligned, the total spin is $F=1 / 2+1 / 2=1$ and the atom is in the higher energy state. When the spins are anti-aligned, the total spin becomes $F=1 / 2-1 / 2=0$, and the atom is in the lower energy state (Griffiths, 1982). The difference in energy between these two states is very small, at $\Delta E=5.84 \times 10^{-6} \mathrm{eV}$. For astronomers, the magic happens when the electron spin flips from parallel to antiparallel, dropping from the higher energy state to the lower one, releasing a photon in the process. This photon has a frequency of $\nu=\Delta E / h=1420.4 \mathrm{MHz}$, where $h$ is the Planck constant. As this frequency lies in the radio regime, astronomers can use radio telescopes to detect $\mathrm{H}$ I. In this thesis, we use the $\mathrm{H}$ I emission line to inform us about the properties of galaxies in the nearby Universe.

Astronomers assume $\mathrm{H}$ I to be optically thin, meaning that the photons emitted in the spin-flip transition do not get absorbed on their way to our detectors. This 
is particularly useful because if we see all the emitted photons, then we can use this to measure the H I mass of the galaxy. We measure the amount of photons using the equation for column density, which gives the number of H I atoms within a cross sectional area of $1 \mathrm{~cm}^{2}$ along the line of sight. The column density is dependent on the brightness temperature of the source, $T_{\mathrm{B}}$, and so we calculate the amount of $\mathrm{HI}$ atoms along the line of sight (l.o.s.) in a galaxy using,

$$
N_{\mathrm{HI}}=1.822 \times 10^{18} \int_{\text {l.o.s. }}\left(\frac{T_{\mathrm{B}}}{\mathrm{K}}\right)\left(\frac{d v}{\mathrm{~km} \mathrm{~s}^{-1}}\right) \mathrm{cm}^{-2},
$$

where $d v$ is the velocity bin over which the temperature is measured. It is worth noting that brightness temperature is not a physical temperature. It is defined as the temperature required for a blackbody to produce the same intensity as the source that is observed. To get to the brightness temperature, we need to take a few steps back to discuss how a radio telescope converts photons to an observable. The feed on the telescope converts photons into electrical currents. The antenna temperature, $T_{A}$, is the temperature of a resistor, which converts power from photons into heat, that would generate the same power per unit bandwidth as the output of the antenna. This antenna temperature is related to the brightness temperature by the ratio of the solid angles of the source being observed $\Omega_{s}$, and the beamwidth of the telescope $\Omega_{b}$,

$$
T_{B}=T_{A}\left(\frac{\Omega_{b}}{\Omega_{s}}\right) .
$$

Now we can measure the mass of a galaxy with the information at hand, using the general relation $M_{H I}=m_{H} \cdot N$. For our purposes, we want to measure $M$ in solar 
masses $M_{\odot}$, so we will convert the mass of the hydrogen atom $m_{H}$ to solar masses. The total number of $\mathrm{H}$ I atoms within the beam size is then $N=\bar{N}_{H I} \cdot A$, where the area of a Gaussian beam at a distance $D$ over a solid angle $\Omega$ is $A=D^{2} \cdot \Omega$. We can rewrite the total mass within the beam size as:

$$
M_{H I}=m_{H} \bar{N}_{H I} D^{2} \Omega
$$

From here, we can convert the brightness temperature in $N_{\mathrm{HI}}$ to flux density, $S_{\nu}$, in units of Janskys using this relation:

$$
T_{B}=\frac{\lambda^{2} S_{\nu}}{2 k \Omega}
$$

Here, $k$ is the Boltzmann constant in units of erg/K. This also allows us to derive the mass independent of the solid angle. After filling in constants and conversions, we have the total mass of the source as,

$$
M_{\mathrm{HI}}=2.36 \times 10^{5}\left(\frac{D^{2}}{\mathrm{Mpc}}\right) \int_{\text {source }}\left(\frac{S_{\nu}}{\mathrm{Jy}}\right)\left(\frac{d v}{\mathrm{~km} \mathrm{~s}^{-1}}\right) \mathrm{M}_{\odot} .
$$

We can use the wavelength of the detected H I to measure the recession velocities and the rotation velocities of galaxies. Hubble's Law, $v=H_{0} D$, tells us the velocity with which the galaxy is moving away from us due to the expansion of the Universe. When we measure the H I emission from a galaxy, we can convert the frequency at the line center to velocity to get recession, or systemic, velocity of the galaxy. Often, the frequencies are Doppler shifted when viewing a galaxy that is at least partially 
inclined. When a rotating galaxy is at some inclination with respect to the viewer, some of the gas is moving toward the observer, and some of the gas is moving away. As this H I moves toward or away relative to the systemic velocity, the emission is Doppler shifted, often creating two peaks from flat rotation where the maximum amount of emission is moving either toward or away from us.

H I can clearly tell us many things about a galaxy, but it can also give us information about the gas within the dark matter halo of a galaxy. We can use the integrated HI line from the disk of a galaxy to determine the mass expected from the kinematics. This is one way in which we can measure the amount of dark matter within a galaxy halo, or the amount of mass that it would take to maintain the rotation that we see in a galaxy. This mass should be similar to the mass of a virialized galaxy. The mass of a dark matter halo that is stable, self-gravitating, and spherical, can be defined by the virial mass,

$$
M_{v i r}=\frac{4 \pi}{3} 200 \rho_{\text {crit }} R_{v i r}^{3}
$$

The virial mass, $M_{v i r}$, is defined at the virial radius $R_{v i r}$, at which the density is some multiple, commonly 200, of the critical density of the Universe, $\rho_{\text {crit }}$, where at $\mathrm{z}=0, \rho_{\text {crit }}=3 H_{0}^{2} / 8 \pi G$. Here, $H_{0}$ is the Hubble constant and $G$ is the gravitational constant. In this thesis, we will calculate both the dynamical masses and virial masses and compare them to better understand their differences, if they exist. 


\subsection{Thesis Outline}

In this thesis, we discuss our observations with single dish telescopes with the purpose of detecting diffuse H I accreting onto a galaxy. Single dish telescopes provide the best brightness sensitivities for which to observe faint gas in the halo of galaxies. Detections of a gaseous halo around a galaxy will bring us closer to understanding the mechanisms which guide galaxy formation and evolution.

In Chapter 2, we present a detailed study of one galaxy as part of the Imaging Galaxies Intergalactic and Nearby Environment (IMAGINE) survey. This observation reached a sensitivity limit of $N_{\mathrm{HI}}=10^{16.16} \mathrm{~cm}^{-2}$ over a $2.6 \mathrm{~km} \mathrm{~s}^{-1}$ channel, the native resolution of the data, making this the deepest map of a nearby galaxy outside of our Local Group, and revealing new structure in the distribution of H I around the galaxy.

In Chapter 3, we will be discussing the observations of 18 MeerKAT H I Observations of Nearby Galactic Objects; Observing Southern Emitters (MHONGOOSE) galaxies with the Green Bank Telescope. These observations reached column density sensitivities of $N_{\mathrm{HI}}=10^{16.75} \mathrm{~cm}^{-2}$ over $6.4 \mathrm{~km} \mathrm{~s}^{-1}$ channels. We also discuss diffuse gas detected in these galaxies.

In Chapter 4, we present an early data release of the IMAGINE survey, with a sub-sample of 18 of the 28 IMAGINE galaxies. These observations are extremely deep, and extend out to or beyond the virial radius of each galaxy. With this information, we are able to investigate the $\mathrm{H}$ in the circumgalactic medium of these galaxies.

We conclude this thesis with Chapter 5, where we review our findings from the 
previous chapters, and discuss the implications of this work. We also present our plans for future work in this field. 


\section{Chapter 2}

\section{Evidence of Ongoing Interaction from IMAGINE Galaxy ESO 214-17}

\subsection{Abstract}

We present the first results from the Imaging Galaxies Intergalactic and Nearby Environment (IMAGINE) survey with the Parkes Radio Telescope. We observed the spiral galaxy, ESO 214-17, as one of a sample of 28 galaxies in the survey. Our observations resulted in the deepest map in H I of a nearby galaxy outside of the Local Group, with a $1 \sigma$ rms noise level of $\sigma_{r m s}=2.8 \mathrm{mK}$, or $4.5 \mathrm{mJy}$, corresponding to a column density of $N_{\mathrm{HI}}=1.3 \times 10^{16} \mathrm{~cm}^{-2}$ over a $2.6 \mathrm{~km} \mathrm{~s}^{-1}$ channel. We surveyed the entire virial volume of ESO 214-17, obtaining a complete census of the H I within its dark matter halo. As a result, we detected one new source in $\mathrm{HI}$, the edge-on galaxy ESO 214-15, which was without previous velocity measurements. We also present evidence of a connection between ESO 214-17 and another member in its group, ESO 214-18. While this is as yet unseen in other wavelengths, we detect a distinct, continuous connection between these two galaxies spatially in velocity, and we believe this is an indication of a bridge between the two galaxies, a possible result of interaction. 


\subsection{Introduction}

How galaxies form and evolve remain one of the most fascinating and fastgrowing areas in astronomy. Specifically, how is gas in the intergalactic medium (IGM) transferred to galaxies, subsequently fuelling star formation, and in what way is this gas accreting onto the galaxies once it enters these galaxies' circumgalactic medium (CGM)? Models of galaxy formation and evolution help us to understand these processes better. We now understand galaxies to be connected through a series of web-like filaments of gas, some of which is shock heated to temperatures of $10^{5}-$ $10^{7} \mathrm{~K}$ through gravitational collapse of the warm-hot intergalactic medium (WHIM) (Davé et al., 1999; Cen \& Ostriker, 1999; Davé et al., 2001; Popping et al., 2015).

Smoothed particle hydrodynamics simulations from Kereš et al. (2005) demonstrate that roughly half of the diffuse gas from the IGM never becomes shock heated to the virial temperature of the galaxy halo. Instead, it radiates its gravitational energy at temperatures below $T \sim 10^{5} \mathrm{~K}$. This is called cold-mode accretion, while the shocked heated gas is accreted via the hot-mode $\left(T \gtrsim 10^{5} \mathrm{~K}\right)$. The trajectory of this gas depends strongly on the mass of the galaxy, where cold-mode accretion

dominates in low mass galaxies (baryonic mass of the galaxy $M_{\text {gal }} \lesssim 10^{10.3} M_{\odot}$ ), and hot-mode accretion dominates in high mass galaxies. The mode of accretion is also shown to be dependent on environment. At low redshift $(z \sim 0)$, there is a clear trend toward cold-mode accretion in low-density environments and hot-mode accretion in high-density environments.

While these theoretical models provide us with a scenario of galaxy formation 
and evolution, observational evidence of these processes is lacking. There has been progress detecting cold-mode accretion via the $\mathrm{Ly} \alpha$ line and other metal lines seen in the spectra of background QSOs (Qu et al., 2019; Cantalupo et al., 2014; Ribaudo et al., 2011; Stocke et al., 2010). These detections give us only one-dimensional information with redshifts, but no information about the morphology or kinematics of the gas in the CGM. To find this information, we must probe the low column density gas in emission through the 21-cm neutral hydrogen line (H I). Popping et al. (2009) show the distribution of H I column densities $\left(N_{\mathrm{HI}}\right)$ necessary to trace accretion processes in galaxy formation. Their simulation probes column densities from $N_{\mathrm{HI}} \sim 10^{14}-10^{21} \mathrm{~cm}^{-2}$, and demonstrate the need to reach at least levels of a few times $10^{18} \mathrm{~cm}^{-2}$ and that the probablility of detection is significantly larger at column densities near $N_{\mathrm{HI}} \sim 10^{17} \mathrm{~cm}^{-2}$. To date, there have been many large surveys mapping the H I around galaxies, however, limitations due to expensive survey time and telescope sensitivity have prevented them from reaching these extremely deep column density sensitivities in galaxies outside of the Local Group, where limitations in galaxy diversity prevent us from making statistical claims on the properties of galaxies. One exception to this is the $5^{\circ} \times 5^{\circ}$ region mapped around M81 $(3.63 \mathrm{Mpc})$ in H I down to $N_{\mathrm{HI}}=1 \times 10^{18} \mathrm{~cm}^{-2}$ (Sorgho et al., 2019a).

Previous single-dish observations of nearby galaxies typically obtain column density detection limits of $N_{\mathrm{HI}}=10^{18-19} \mathrm{~cm}^{-2}$ (Westmeier et al., 2017; Pingel et al., 2018; Sorgho et al., 2019b). Large surveys, such as HIPASS (Meyer et al., 2004), ALFALFA (Haynes et al., 2011), and EBHIS (Winkel et al., 2016), cover a large area of sky, but at high column densities and coarse velocity resolution, whereas 
high-resolution surveys like The HI Nearby Galaxy Survey (THINGS; Walter et al., 2008) mapped the structure of nearby galaxies using the Very Large Array, but with the focus on high spatial resolution, only reached $N_{\mathrm{HI}}$ sensitivities of a few times $10^{20} \mathrm{~cm}^{-2}$. On a case-by-case basis, only the nearest galaxies are mapped down to lower column densities. Within the Local Group of galaxies, single dish telescopes provide not only excellent sensitivity, but also good spatial resolution. Our closest neighbors, M31 and M33, have been mapped to a column density of a few times $10^{17} \mathrm{~cm}^{-2}$, revealing a bridge between the two galaxies (Braun \& Thilker, 2004). Wolfe et al. (2013) revealed this bridge to be made up of clumpy clouds of H I, rather than a smooth stream. Outside of the Local Group we can begin to quantify the gas in galaxies on a statistical basis. Individual galaxies have been targeted and mapped to these same very low column densities, such as Mihos et al. (2012), mapping M101 in H I with a $1 \sigma$ column density sensitivity of $N_{\mathrm{HI}}=6.3 \times 10^{16} \mathrm{~cm}^{-2}$ over a $5.2 \mathrm{~km} \mathrm{~s}-1$ channel, but there have been no large surveys providing a statistical picture of galaxies by probing both deep in column density and in the volume of the CGM around these galaxies.

In this paper, we present the results of our observations which are both extremely deep and extend out to the virial radius of the central galaxy, probing the entire volume of the dark matter halo of the spiral galaxy ESO 214-17. These observations are part of a larger survey which expands to a sample of 28 nearby spiral galaxies, obtaining both single-dish and interferometric data for each. The Imaging Galaxies Intergalactic and Nearby Environment (IMAGINE ${ }^{1}$ ) survey is a 2261 hour legacy survey of 28 spiral galaxies using the Australia Telescope Compact Array 
(ATCA) in all compact configurations. At 96 hours per galaxy, mapping the area around each galaxy out to $\lesssim 1$ square degree $(\mathrm{r} \leq 150 \mathrm{kpc}$ ), this legacy survey will reach $1 \sigma$ column density sensitivities of $\sim 2.5 \times 10^{17} \mathrm{~cm}^{-2}$ over a $20 \mathrm{~km} \mathrm{~s}^{-1}$ linewidth, an unprecedented sensitivity level for a large survey. The sample of galaxies chosen provide us with a range of galaxy properties, enabling us to make statistical claims about spiral galaxies. Galaxy distances in the sample range from 1.8 - 17.7 Mpc. Each galaxy is actively star-forming, containing HI masses between $M_{\mathrm{HI}} \sim 10^{8-10}$ $M_{\odot}$, and resides in a range of environments from isolated to groups. The goals of this project are to quantify the mass, structure, and column density distribution in the CGM of each galaxy, to determine how the H I properties of the gas depend on the environment, and measure the neutral gas infall rate at $z=0$ as a function of galaxy properties.

For the single dish component of IMAGINE, we are using the 64-meter Parkes Radio Telescope to measure the total H I content of the galaxy and the dark matter halo. In order to do this, we are going beyond the physical limits of previous H I surveys to conduct a focused study on the entire volume within the virial radius of each galaxy in the sample, obtaining the total H I gas content within the dark matter halo of each galaxy. This will enable us to connect H I features seen close to the galaxy disk with ATCA to the IGM. Additionally, for each galaxy in our large sample, we are mapping down to column densities of $N_{\mathrm{HI}} \sim 10^{17} \mathrm{~cm}^{-2}$ for a $5 \sigma$ detection over a $20 \mathrm{~km} \mathrm{~s}^{-1}$ line width, nearly an order of magnitude deeper than any previous survey. This sensitivity will enable us to discover new morphological

\footnotetext{
${ }^{1}$ http://www.imagine-survey.org/
} 
features in $\mathrm{H}$ I and quantify the kinematics of any H I gas in the CGM of these galaxies, with the potential of identifying gas falling onto the galaxy via cold-mode accretion. Moreover, we will get a complete picture of the $\mathrm{HI}$ at low $N_{\mathrm{HI}}$ around galaxies by filling in the missing short-spacing information from extended emission missed by an interferometer. This low $N_{\mathrm{HI}}$ gas is potentially connecting galaxies to their satellites, but has mostly remained undetected due to the sensitivity limits of other surveys. With our highly sensitive observations of a large sample of galaxies, we will be able to quantify or set a limit on the amount of gas possibly connecting these structures. To date, Parkes-IMAGINE has obtained 218 of our requested 395 hours of data, completing 21 out of 28 galaxies in our sample.

Our central target, ESO 214-17, is described in Section 2 of this paper. Details of our observations and how we reduced our data are laid out in Section 3. Our results are presented in Section 4, and we discuss implications of these results in Section 5. Conclusions and our future work are discussed in Section 6.

\subsection{Target}

Our target, ESO 214-17 was chosen as part of the IMAGINE sample of spiral galaxies in the nearby universe. We assumed a distance of $13.1 \mathrm{Mpc}$ (Tully, 1988), a dark matter halo mass of $M_{\text {halo }}=10^{11.1} M_{\odot}$ estimated using the Moster et al. (2010) stellar-halo mass relation, virial radius of $128 \mathrm{kpc}$, and galaxy volume density of 0.10 


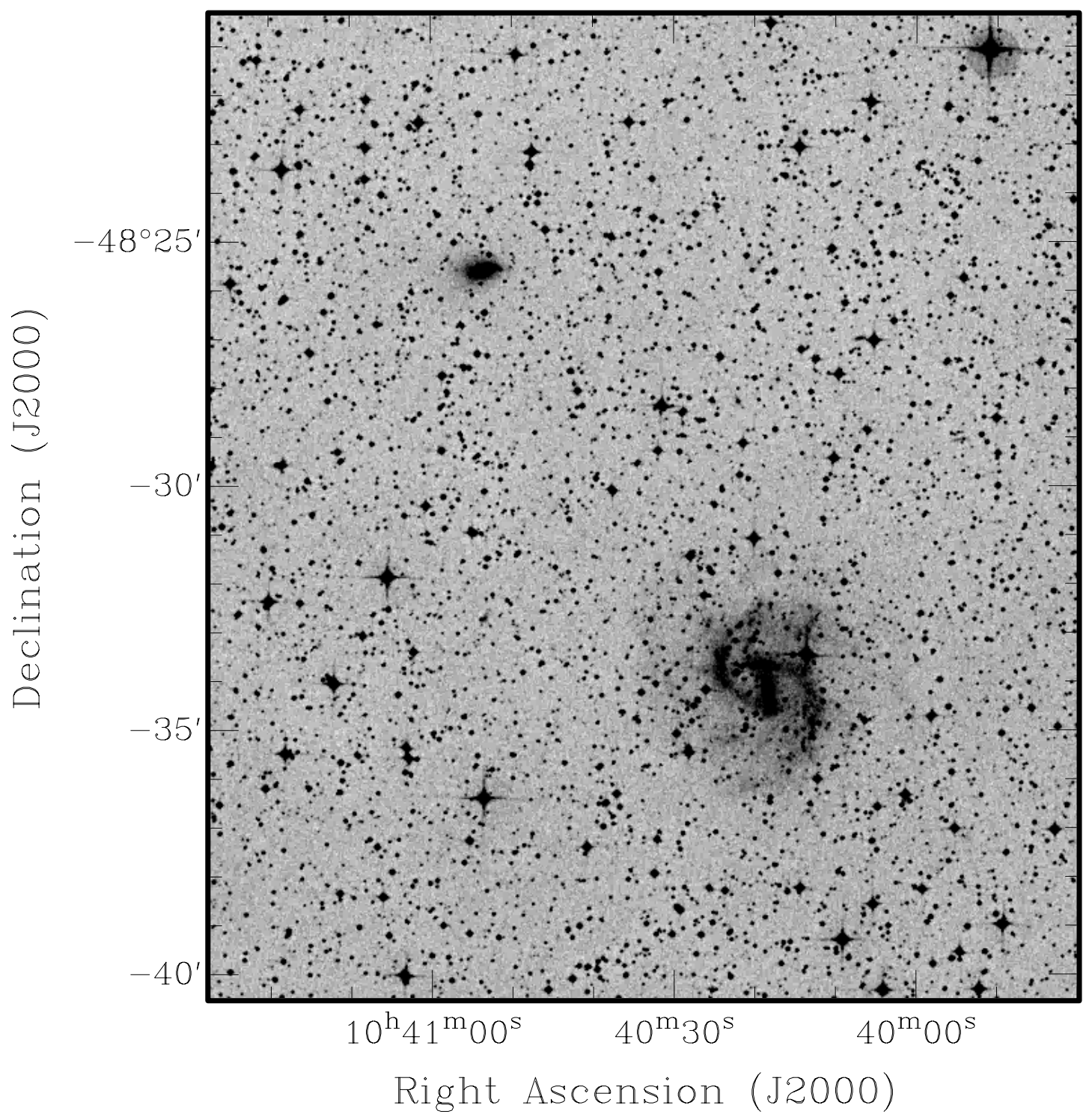

Figure 2.1: Optical image in $\mathrm{B}_{\mathrm{j}}$ band from the Digital Sky Survey II (DSS2). ESO 21417 is the face-on spiral galaxy in the lower right. Companion galaxy, ESO 214-18, to the northeast of ESO 214-17, where east is to the left. There is no indication in the optical image that the two galaxies are connected. 
$\mathrm{Mpc}^{-3}$ (Nearby Galaxy Catalog: Tully (1988)). We calculated the virial radius using,

$$
R_{v i r}=\left(\frac{3 M_{v i r}}{4 \pi 200 \rho_{c}}\right)^{1 / 3} .
$$

We define $R_{v i r}$ as the radius enclosing an overdensity of 200, as suggested by simulations, times the critical density of the universe, where $M_{v i r}$ is the mass of the dark matter halo, and $\rho_{c}$ is the critical density of the universe. In relation to the full sample of IMAGINE galaxies, ESO 214-17 is among the lowest in halo mass and density environment, making it a prime candidate for searching for diffuse gas flowing into the galaxy via cold-mode accretion. It is classified as an $\mathrm{SBcd}_{\text {galaxy }}{ }^{2}$, is seen at an inclination of $36^{\circ}$, and displays no known interaction at optical wavelengths, as can be seen in Figure 2.1.

The group environment is not particularly well agreed upon for ESO 214-17. Kourkchi \& Tully (2017) catalogue ESO 214-17 as a group galaxy in a group with ESO 264-35 (an angular separation of 1.04 degrees, or $237 \mathrm{kpc}$ at a distance of 13.1 Mpc). According to the ESO/Uppsala survey of the ESO(B) atlas (Lauberts, 1982) and the NASA/IPAC Extragalactic Database $\left(\mathrm{NED}^{3}\right)$, it is the largest member of a group containing only itself and the galaxy ESO 214-18. It is also listed in the Nearby Galaxy Catalog Tully (1988) with a local galaxy density of $0.10 \mathrm{Mpc}^{-3}$. For this paper, we put an additional focus on ESO 214-18, an Sbc galaxy with an inclination of $67.6^{\circ}$, and a projected distance from ESO 214-17 of $38.6 \mathrm{kpc}$ using a distance of $13.1 \mathrm{Mpc}$ and an angular separation of 0.17 degrees. These values are listed in Table 2.1. With

\footnotetext{
${ }^{2} \mathrm{http}: / /$ leda.univ-lyon1.fr/

${ }^{3}$ http://ned.ipac.caltech.edu/
} 
Table 2.1: Target and Companion

\begin{tabular}{lcc}
\hline \hline & & \\
ESO 214-17: & & \\
Type & SBcd & \\
Inclination & 36 & $\mathrm{deg}$ \\
Galaxy density & 0.10 & $\mathrm{Mpc}^{-3}$ \\
$D_{25}$ & 5.2 & $\operatorname{arcmin}$ \\
& & \\
\hline & & \\
ESO 214-18: & & \\
Type & $\mathrm{Sbc}$ & \\
Inclination & 67.6 & $\mathrm{deg}$ \\
$D_{25}$ & 1.3 & $\operatorname{arcmin}$ \\
& & \\
\hline \hline
\end{tabular}

Table 2.2: Notes. Galaxy density from Nearby Galaxy Catalog Tully (1988). Inclination angle from face-on, morphological type, and the optical diameter, $D_{25}$, measured at the $25^{\text {th }}$ mag $\operatorname{arcsec}^{-2}$ isophote in the B-band values taken from http://leda.univlyon1.fr/

our ultra-deep observations, we will obtain a more complete census of the H I within the virial radius of the galaxy, which will provide us with a clearer picture of the group environment of ESO 214-17.

Neither ESO 214-17 nor ESO 214-18 have previously been the focus of any individual studies, but have both been detected in several wavelengths across many surveys including H I from HI Parkes All Sky Survey (HIPASS) (Koribalski et al., 2004), near-IR from the Two-Micron All Sky Survey (2MASS) (Skrutskie et al., 2006), and in many optical surveys such as the Digital Sky Survey (DSS) (Lasker et al., 1990), which can be seen in Figure 2.1. Neither galaxy have been deemed remarkable in other surveys, but were both flagged as confused sources in HIPASS. Not surprisingly, our focused study of ESO 214-17 has revealed new, interesting details about the galaxy. 
Table 2.3: Observing Parameters

\begin{tabular}{lcc}
\hline \hline Parameter & Value & Units \\
$\Delta v^{1}$ & 2.6 & $\mathrm{~km} / \mathrm{s}$ \\
Bandwidth & 200 & $\mathrm{MHz}$ \\
Aperture efficiency & 0.7 & \\
Gain & 1.1 & $\mathrm{Jy} / \mathrm{K}$ \\
Beam FWHM & 14.4 & $\operatorname{arcmin}$ \\
$\mathrm{B}_{\text {maj }}{ }^{2}$ & 864 & $\operatorname{arcsec}$ \\
$\mathrm{B}_{\text {min }}{ }^{3}$ & 864 & $\operatorname{arcsec}$ \\
Mapsize & $2 \times 2$ & $\mathrm{deg}$ \\
& $227 \times 227$ & $\mathrm{kpc}$ \\
Total integration time & 52 & $\mathrm{hrs}$ \\
Integration time & 4 & $\mathrm{hrs} / \mathrm{beam}$ \\
$\sigma_{\text {rms }}{ }^{1 \sigma N_{\mathrm{HI}}}{ }^{5}$ & 4.5 & $\mathrm{mJy}$ \\
\hline \hline
\end{tabular}

Table 2.4: ${ }^{1}$ Velocity resolution. ${ }^{2}$ Major axis of the beam. ${ }^{3}$ Minor axis of the beam. ${ }^{4}$ Measured rms noise. ${ }^{5}$ Column density sensitivity per $2.6 \mathrm{~km} \mathrm{~s}^{-1}$ channel

\subsection{Observations and Reduction}

\subsubsection{Observations}

We collected our data using the 20-cm Parkes multibeam receiver (StaveleySmith et al., 1996), which contains 13 feed horns laid into a hexagonal shape, greatly reducing our survey time. Each of the 13 beams has a full width at half-power angular resolution of $14.4^{\prime}$ at $1.42 \mathrm{GHz}$. Our native velocity resolution is $2.6 \mathrm{~km} \mathrm{~s}^{-1}$ from 16384 channels over a $200 \mathrm{MHz}$ bandwidth. In order to avoid solar interference, our observations were only taken at night.

We scanned over our selected region in right ascension and declination, creating a basket-weave pattern in order to fill in gaps and reduce scanning artifacts. We integrated every 2 seconds, with a total integration time of 4 hours, completing a 
$2^{\circ} \times 2^{\circ}$ map of ESO 214-17 past the virial radius of the galaxy. The map was completed in one night on 25 July 2017, using the flux calibration source PKS 1934638, which has a well characterized flux model giving a flux density of $14.9 \mathrm{Jy}$ at $1420 \mathrm{MHz}$. The total integration time resulted in an average $1 \sigma \mathrm{rms}$ noise of $4.5 \mathrm{mJy}$, corresponding to $2.8 \mathrm{mK}$ at a velocity resolution of $2.6 \mathrm{~km} \mathrm{~s}^{-1}$. This $1 \sigma$ per channel level corresponds to a column density of $N_{\mathrm{HI}}=1.35 \times 10^{16} \mathrm{~cm}^{-2}$ and $M_{\mathrm{HI}}=6.7 \times 10^{5}$ $M_{\odot}$. Our observation parameters are listed in Table 2.3.

\subsubsection{Data Reduction}

The data from all observations has been reduced using a reduction pipeline we created utilizing LIVEDATA $^{4}$, GRIDZILla ${ }^{4}$, MIRIAD $^{4}$, Python, and our own shell scripts. Our data processing was performed in LIVEDATA, where we masked out Galactic emission before fitting the baselines with a robust first-order polynomial. We iterated once over this first-order robust polynomial, with a deviation of three, discarding points outside this number of median deviations. We followed this with bandpass-removal, and application of a Hanning filter to remove Gibbs ringing from Galactic emission.

Each scan was combined and gridded into a data cube with the gridding program GRIDZILLA. This program assigns each spectrum to an appropriate pixel, using a particular weighting scheme while discarding spectra with corrupt data. Once this is done, it calculates the value contained in each pixel. We set our cutoff radius, which determines the number of spectra contributing to the calculation of each pixel

\footnotetext{
${ }^{4}$ https://www.atnf.csiro.au/computing/software/
} 
value, to be $6^{\prime}$ and pixel sizes in each image to be $4^{\prime} \times 4^{\prime}$ in order to maximize image resolution while minimizing image noise, as per Barnes et al. (2001). We used the weighted median-gridding algorithm for its robustness in removing corrupted data, while discarding $1 \%$ of the data during the smoothing operation. We selected a gridding kernel function of a top-hat with a FWHM of $12^{\prime}$. Our resulting data cube contains two spatial axes in RA and Dec., with the z-axis in frequency/velocity. The cube spans a velocity range of $-500-2000 \mathrm{~km} \mathrm{~s}^{-1}$, extracted from our total bandwidth of $200 \mathrm{MHz}$, which covers all emission associated with galaxies in the IMAGINE sample.

A visual inspection by multiple co-authors was performed on the data in order to search for other sources of H I emission. Any potential sources were either confirmed and identified using the NASA/IPAC Extragalactic Database (NED) and through catalogue searches with VizieR ${ }^{5}$, or were discarded after a number of tests could not confirm their astrophysical nature. One of the ways we tested this was to compare the central velocity of each potential source with the central velocity of the bright central galaxy. If these velocities lined up perfectly, without any offset, we determined those to be artificial in nature and a likely result of the reduction process. Four sources were discarded this way, and three additional sources were determined to be real detections.

\footnotetext{
${ }^{5}$ http://vizier.u-strasbg.fr/
} 
Table 2.5: Properties of galaxies detected in H I

\begin{tabular}{|c|c|c|c|c|c|c|c|c|c|c|c|}
\hline \multirow[t]{2}{*}{ Source } & R.A. (2000.0) & Decl. (2000.0) & $\mathrm{D}$ & $\mathrm{V}_{\mathrm{sys}}$ & $\mathrm{W}_{20}$ & $\mathrm{~W}_{50}$ & $\mathrm{~S}_{\mathrm{HI}}$ & $\log _{10} \mathrm{M}_{\mathrm{HI}}$ & $\mathrm{S} / \mathrm{N}$ & $\sigma_{r m s}$ & $\log _{10} \mathrm{M}_{\mathrm{dyn}}$ \\
\hline & hh:mm:ss.s & dd:mm:ss.s & $\mathrm{Mpc}$ & $\mathrm{km} \mathrm{s}^{-1}$ & $\mathrm{~km} \mathrm{~s}^{-1}$ & $\mathrm{~km} \mathrm{~s}^{-1}$ & $\mathrm{Jy} \mathrm{km} \mathrm{s}^{-1}$ & $M_{\odot}$ & & mJy & $M_{\odot}$ \\
\hline$(1)$ & $(2)$ & $(3)$ & $(4)$ & $(5)$ & $(6)$ & $(7)$ & $(8)$ & $(9)$ & $(10)$ & $(11)$ & $(12)$ \\
\hline ESO 214-17 & 10:40:18.73 & $-48: 34: 10.9$ & 14.7 & 1071 & 46 & 30 & 76.99 & 9.49 & 536 & 4.4 & 9.78 \\
\hline ESO 214-18 & $10: 40: 54.09$ & $-48: 25: 38.3$ & 13.6 & 998 & 93 & 61 & 8.04 & 8.51 & 80.9 & 4.3 & 9.40 \\
\hline ESO 264-35 & $10: 42: 50.49$ & $-47: 37: 03.5$ & 15.2 & 1109 & 153 & 133 & 7.18 & 8.59 & 13.5 & 6.4 & 9.13 \\
\hline ESO 214-15 & $10: 36: 51.93$ & $-47: 47: 59.8$ & 2.7 & 248 & 57 & 44 & 75.03 & 8.11 & 27.8 & 10.8 & 8.12 \\
\hline
\end{tabular}

Table 2.5: Columns 2 and 3 taken from NED. Column 4 distances derived from Hubble's law using $H_{0}=73 \mathrm{~km} \mathrm{~s}^{-1} \mathrm{Mpc}^{-1}$, with the exception of ESO 214-15, which we calculate using the Masters et al. (2014) K-band Tully-Fisher relation. Column 5 is the systemic velocity of the galaxy obtained from our robust moment 1 (velocity) measurement. Columns 6 and 7 are the $20 \%$ and $50 \%$ maximum linewidths from the spectral fit. Column 8 is our total integrated flux. Column 9 is our total H I mass. Column 10 is the peak signal-to-noise ratio of the detection. Column 11 is the $1 \sigma \mathrm{rms}$ noise per $2.6 \mathrm{kms}^{-1}$ channel. Column 12 is the dynamical mass calculated using the $20 \%$ linewidth, corrected for inclination, and the H I diameter as described in Section 4. ESO214-18 properties were derived from our Gaussian fit of the spectra. 


\subsection{Results}

\subsubsection{Spectra}

We used the MIRIAD task MBSPECT to generate integrated spectra for each source. In order to generate these spectra, a first-order polynomial was fitted to emission-free regions of the baseline with the purpose of removing baseline variations from each spectrum. This task also performs a spectral fit to the integrated spectra, producing total integrated flux values. The total integrated H I profiles of ESO 21417 and ESO 214-18 are shown in Figure 2.2 with regions integrated over marked by vertical lines. Since the two galaxies, ESO 214-17 and ESO 214-18, are connected in velocity space, we chose to use a velocity of $1040 \mathrm{~km} \mathrm{~s}^{-1}$ as the velocity cutoff for each galaxy, where velocities below this cutoff were assumed to be associated with ESO 214-18, and velocities above this cutoff associated with ESO 214-17. ESO 21417 displays a clear Gaussian profile which can be easily measured with MBSPECT, however, it was not able to make these measurements on the spectrum of ESO 21418. Instead, we fit two separate Gaussians to the total integrated spectrum of the two galaxies and obtained H I masses for each, and $20 \%$ and 50\% maximum points for both Gaussian profiles. These are listed in Table 2.5.

\subsubsection{Galaxy Properties}

We calculate our values of column density, $N_{\mathrm{HI}}$ using 
ESO214-17

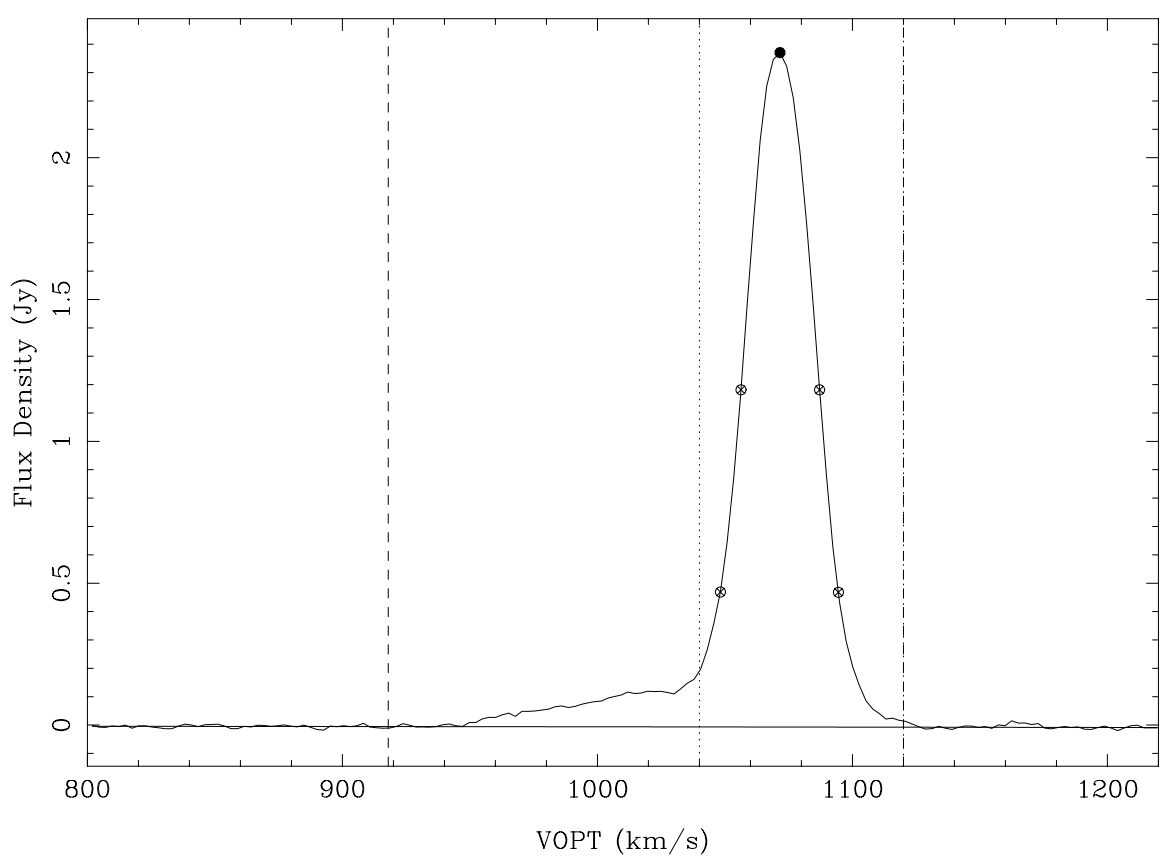

ESO214-18

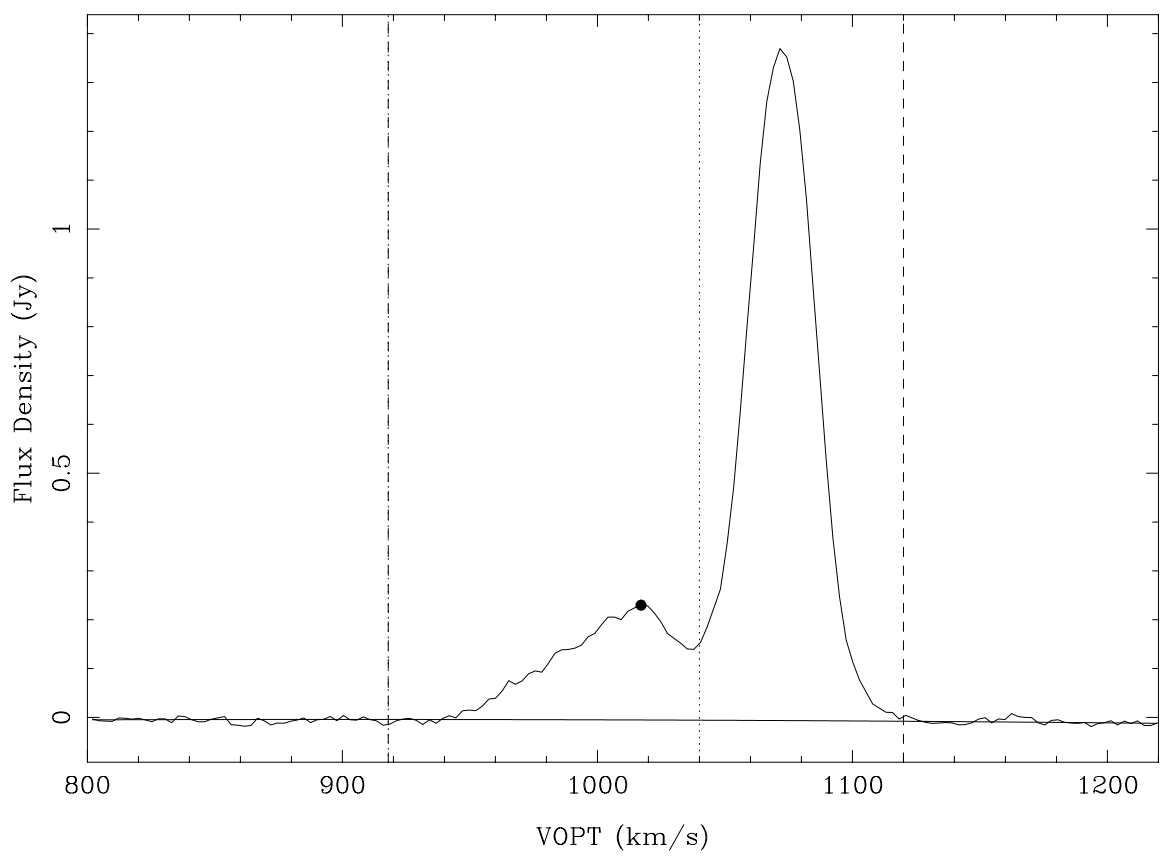

Figure 2.2: Global integrated H I profiles of ESO 214-17 and ESO 214-18 from the Parkes data. Emission from ESO 214-17 (upper panel) and ESO 214-18 (lower panel) is measured over the regions between the dashed and dotted vertical lines. The open circles and $\times$ 's indicate the width-maximized (measured from outside the profile to inside) and minimized (measured from the peak, out) $20 \%$ and $50 \%$ velocity widths, respectively. The filled circle represents the peak value of the specified profile, which is measured between the vertical lines where masking of the alternating galaxy is indicated by the dotted line. 


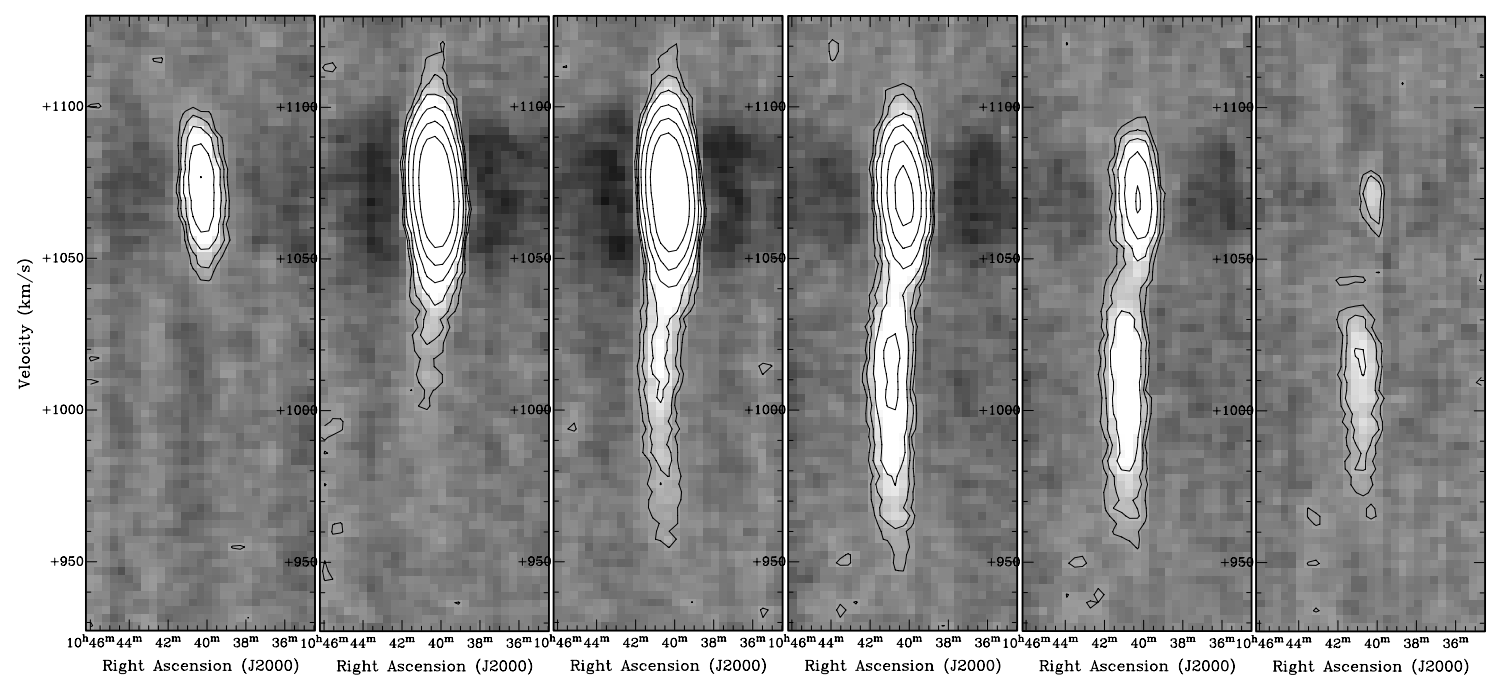

Figure 2.3: H I position-velocity slice of ESO 214-17 (around $\sim 1070 \mathrm{~km} \mathrm{~s}^{-1}$ ) as it smoothly connects to ESO 214-18 (around $\sim 1010-1020 \mathrm{~km} \mathrm{~s}^{-1}$ ). There is a continuous bridge between throughout several panels, where the contours of the bridge are the 5, 10, and 20 times the rms noise of $0.0045 \mathrm{Jy} /$ beam or $2.8 \mathrm{mK}$. Contours throughout are shown at 5, 10, 20, 40, 80, and $160 \sigma$, correlating to column density levels of $N_{\mathrm{HI}}=0.68,1.4,2.7,5.4,10.8$, and $21.7 \times 10^{17} \mathrm{~cm}^{-2}$.

$$
N_{\mathrm{HI}}=1.822 \times 10^{18}\left(\frac{T_{\mathrm{B}}}{\mathrm{K}}\right)\left(\frac{d v}{\mathrm{~km} \mathrm{~s}^{-1}}\right) \mathrm{cm}^{-2} .
$$

Here, the brightness temperature, $T_{\mathrm{B}}$, is derived assuming a gain of $1.1 \mathrm{Jy} \mathrm{K}^{-1}$ with an aperture efficiency of 0.7 at $1420 \mathrm{MHz}^{7}$. We use one of two $d v$ values when calculating our column densities. We use the resolution of our data, $2.6 \mathrm{~km} \mathrm{~s}^{-1}$, to calculate the $1 \sigma N_{\mathrm{HI}}$ value per channel, and a linewidth of $20 \mathrm{~km} \mathrm{~s}^{-1}$, the minimum linewidth we expect to see an $\mathrm{H}$ I detection, to calculate the $5 \sigma N_{\mathrm{HI}}$ detection level.

The projected distance between the two sources is $39 \mathrm{kpc}$ from the centers of

\footnotetext{
${ }^{7}$ Parkes Users Guide:

https://www.parkes.atnf.csiro.au/observing/documentation/
} 
each galaxy. A visual inspection of the optical DSS images of the two galaxies do not reveal perturbations of either galaxy, however the H I reveals a distinct overlap in velocity space, indicating a possible connection or gas interaction between the two galaxies. This can be best seen in Figure 2.3. This position-velocity diagram highlights the smooth connection of ESO 214-17 to the companion, ESO 214-18. The companion appears slightly offset in RA position, while remaining connected to the central galaxy in velocity. Contours on the bridge between the two galaxies are at 5,10 , and $20 \sigma$, demonstrating the high confidence of this continuous gas distribution. While it is possible that some of the diffuse emission we are seeing could be coming from stray-radiation, we do not believe it could realistically account for the amount of $\mathrm{H}$ I that we see in these diffuse regions because of the high significance of the detections.

The H I envelope around the two galaxies can be seen in the channel map of Figure 2.4 showing the gas in slices through velocity. Central points of both ESO 21417 and ESO 214-18 are indicated with the cross hair markers. The gas in the lower velocity channels smoothly transitions from ESO 214-18 to ESO 214-17 in the higher velocity channels, with no distinct break from one galaxy to the next.

We derive the H I mass of the two galaxies individually and as a unit, using the total integrated flux measurement from the spectra shown in Figure 2.2. Assuming the $\mathrm{HI}$ is optically thin, we calculated the $\mathrm{H}$ I mass using,

$$
M_{\mathrm{HI}}=2.36 \times 10^{5} D^{2} \int_{v_{1}}^{v_{2}} S(v) d v \mathrm{M}_{\odot},
$$



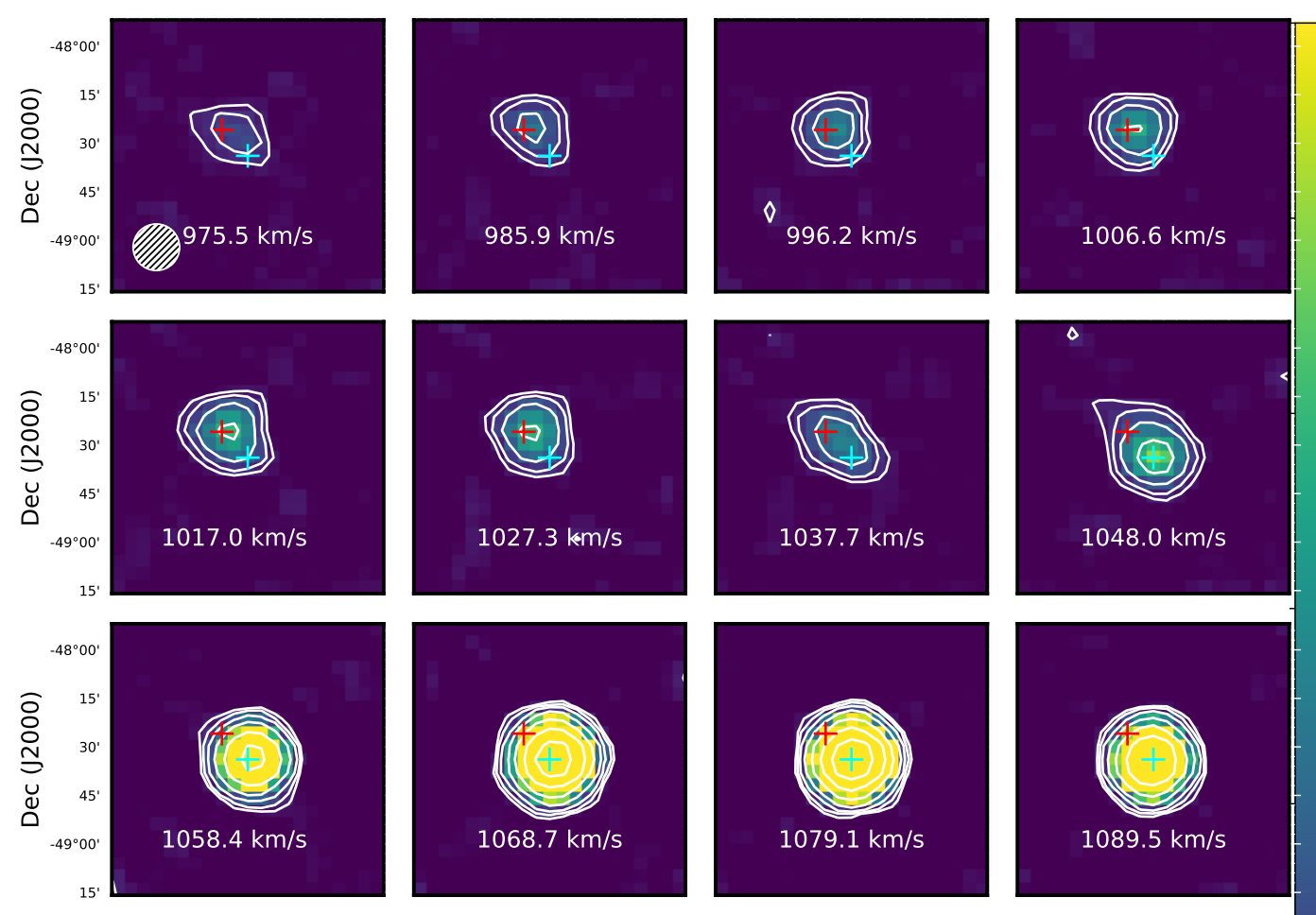

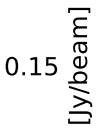
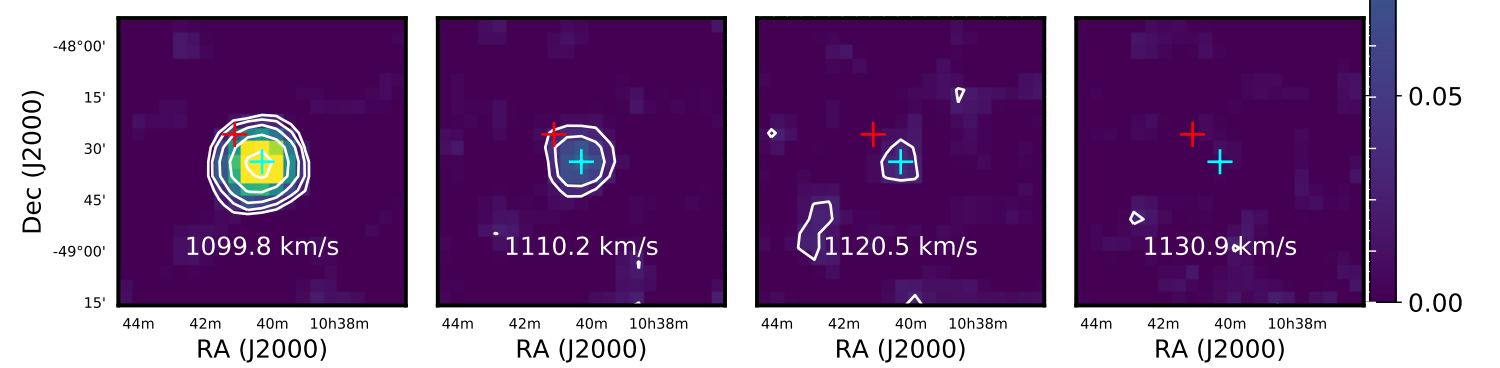

Figure 2.4: Channel maps of the central portion of the Parkes data cube, encompassing both ESO 214-17 and ESO 214-18, at 14.3' spatial resolution, with each channel from $975.5 \mathrm{~km} \mathrm{~s}^{-1}$ to $1130.9 \mathrm{~km} \mathrm{~s}^{-1}$ separated by $10.4 \mathrm{~km} \mathrm{~s}^{-1}$. The Parkes beam size is shown in the top left panel as the hatched circle. The measured rms noise in the cube is $\sigma_{r m s}=4.5 \mathrm{mJy}(2.8 \mathrm{mK})$. The barycentric recessional velocity is shown in each panel. The red cross marks the center of ESO214-18, which appears north-east, where east is to the left, of ESO 214-17, indicated in cyan. Contours are shown at 5, 10, 20, 40, 80, 160, and $320 \sigma$, correlating to column density levels of $N_{\mathrm{HI}}=0.68,1.4,2.7,5.4,10.8,21.7$, and $43.4 \times 10^{17} \mathrm{~cm}^{-2}$. 
where $D$ is the distance to the source in Mpc and $\int_{v_{1}}^{v_{2}} S(v) d v$ is the integrated H I flux over the source velocities, $v_{1}$ to $v_{2}$, with units of $\mathrm{Jy} \mathrm{km} \mathrm{s}^{-1}$ (Giovanelli \& Haynes, 1988). The HI masses of ESO 214-17 and its companion, ESO 214-18, are $M_{\mathrm{HI}}=$ $3.12 \times 10^{9} \quad M_{\odot}$ and $=3.26 \times 10^{8} \quad M_{\odot}$, respectively. Using the total integrated flux from the fit of their combined spectra, we derive an $M_{\mathrm{HI}}=3.45 \times 10^{9} M_{\odot}$. These values, and other derived galaxy properties are listed in Table 2.5.

We use the H I integrated profile linewidths to calculate the total dynamical mass inside the galaxy. We estimate the total dynamical masses inside of radius, r, for each galaxy using

$$
M_{\mathrm{dyn}}=2.3 \times 10^{5}\left(\frac{v_{\text {rot }} / \sin (i)}{\mathrm{km} \mathrm{s}^{-1}}\right)^{2}\left(\frac{r}{\mathrm{kpc}}\right) \mathrm{M}_{\odot}
$$

where $v_{\text {rot }}=\mathrm{W}_{20} / 2$, and $i$ is the inclination of the galaxy in degrees. We calculated the radius using the Broeils \& Rhee (1997) scaling relation of the H I diameter $\left(D_{\mathrm{HI}}\right)$ :

$$
\log D_{\mathrm{HI}}=(1.00 \pm 0.03) \log D_{25}+(0.23 \pm 0.04)
$$

with the optical diameter $\left(D_{25}^{6}\right)$ measured at the $25^{\text {th }}$ mag $\operatorname{arcsec}^{-2}$ isophote in the B-band.

Using these HI mass estimates, we can calculate both the neutral gas fraction $\left(M_{\mathrm{HI}} / M_{d y n}\right)$ and the mass ratio of the satellite galaxy to the main galaxy: $\mathrm{M}_{\mathrm{H} \text { I,sat }} / \mathrm{M}_{\mathrm{HI} \text {,main }}=0.10$. This mass ratio of the main to the satellite, $9.6: 1$, would typically be defined as a minor merger as they are not comparable in mass, and can

\footnotetext{
${ }^{6}$ http://leda.univ-lyon1.fr/
} 
be used later to inform us about enhancement of star formation in the satellite galaxy, which along with the gas fraction could in turn inform us about the time-scale of a potential merger (Bell et al., 2006; Lotz et al., 2010).

The panels in Figures 2.5 and 2.6 show the contours from the integrated H I (moment 0) map of both ESO 214-17 and ESO 214-18 overlaid on an optical DSS image of the two galaxies, along with the corresponding velocity (moment 1) and velocity dispersion (moment 2) maps for both galaxies. Because we are looking for diffuse emission, we used a blanking threshold of $1.5 \sigma$ to create the moment 0 map. Blanking pixels at a higher cutoff point would potentially cut out diffuse emission from the galaxy. For each galaxy, ESO 214-17 and ESO 214-18, we created moment 0 maps by integrating pixel values in the data cube over the channel range containing emission from the galaxies, to illustrate the emission coming from ESO 214-18. Contours from both galaxies' moment 0 maps were overlaid onto an optical DSS2 image in Figure 2.5, and onto the velocity and dispersion maps in Figure 2.6. The offset in velocity distribution seen in the top panel of Figure 2.6 is likely due to the difference in inclination between the nearly face-on ESO 214-17 and the $36^{\circ}$ inclination of ESO 21418. It could also be an indication of the H I between the two galaxies interacting. This offset is exaggerated in the velocity dispersion map in the bottom panel, where again, most of the movement is seen from the inclined companion. 


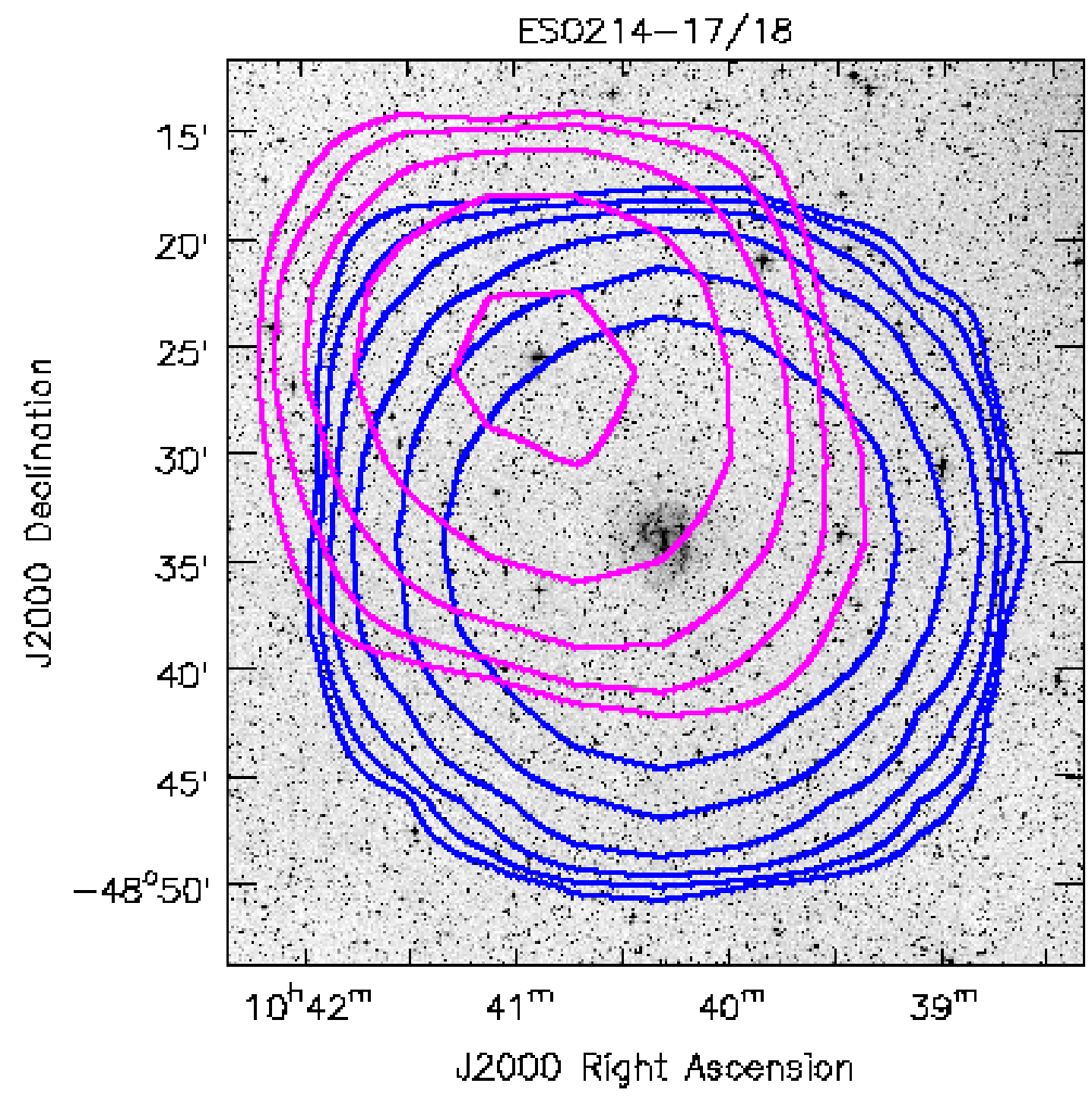

Figure 2.5: Integrated intensity map (moment 0) of both ESO 214-17 and ESO 214-18 overlaid onto an optical DSS2 Blue image. Contours for ESO 214-18 are shown in magenta. Contours for ESO 214-17 are shown in blue. The dashed line is the lowest contour taken from the combined moment 0 map of ESO 214-17 and ESO 214-18. All contours are at levels of 10, 20, 40, 80, 160, and 320 $\sigma$, where the integrated rms noise value we used was $0.06 \mathrm{Jy} /$ beam, as described in Section 4.2. 

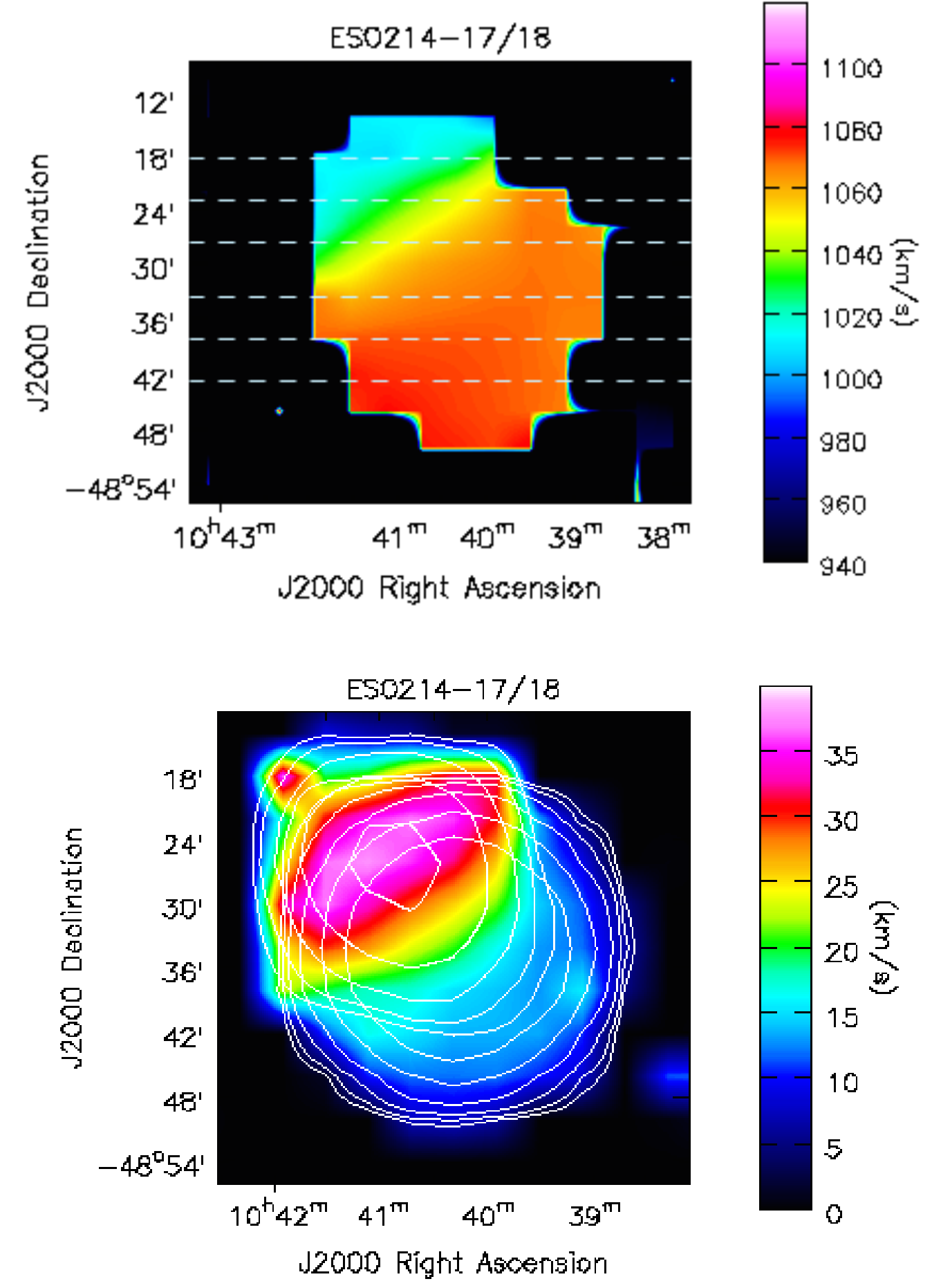

Figure 2.6: Top: Velocity (moment 1) map of ESO214-17 with ESO214-18. Dashed horizontal lines indicate the declination position of the velocity slices shown in Figure 2.3. Bottom: Velocity dispersion (moment 2) map. Contours are shown at 10, 20, 40, 80, 160, and $320 \sigma$. This image shows a distinct difference in the kinematic distribution of the two connecting galaxies. 

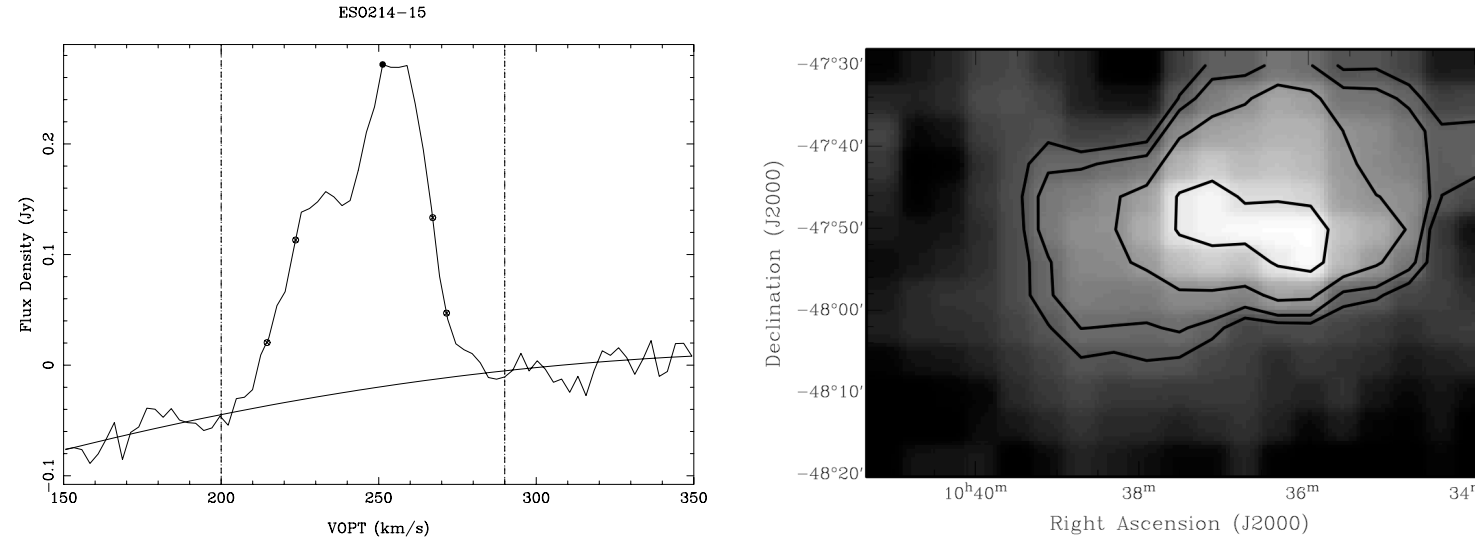

Figure 2.7: Upper: Same as Figure 2.2, but for total integrated spectra of ESO 21415. Lower: Integrated intensity map of ESO 214-15 with contours at 10, 20, 30, and $40 \sigma$.

\subsubsection{Other H i sources in the cube}

We performed a visual inspection of the cube, detecting three sources apart from ESO 214-17, one of which did not have previous H I detections. We identified and confirmed our second source emitting in H I, ESO 214-18, revealing a connection to ESO 214-17 in velocity space. This emission spans at least 40 channels, over a velocity region of $918 \mathrm{~km} \mathrm{~s}^{-1}$ to $\sim 1040 \mathrm{~km} \mathrm{~s}^{-1}$, where the emission begins to overlap with ESO214-17 ( $1040 \mathrm{~km} \mathrm{~s}^{-1}$ to $1120 \mathrm{kms}^{-1}$ ) (see Figure 2.2). We also detected the spiral galaxy ESO 264-35 at a $13.8 \sigma$ level, which can be seen in Figure 2.8. We identified both sources using their positions with a search of the NASA/IPAC Extragalactic Database.

A fourth galaxy, which we believe to be spiral edge-on galaxy ESO 214-15 (Figure 2.8), was identified with a velocity of $249 \mathrm{~km} \mathrm{~s}^{-1}$. This is the first known 


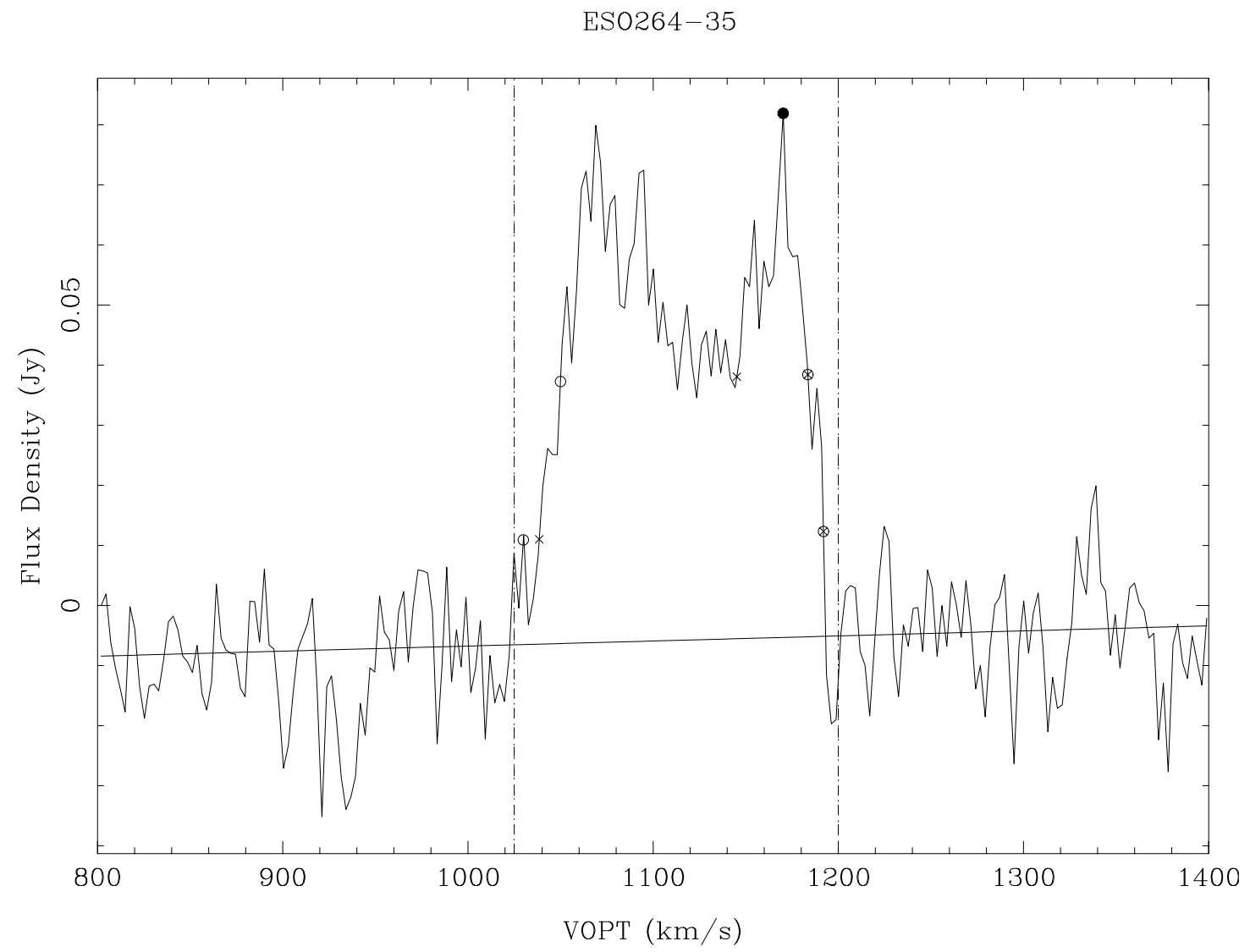

Figure 2.8: Same as Figure 2.2, but for total integrated spectra of ESO 264-35. 
velocity measurement of this galaxy. There is currently no known distance or redshift information for ESO 214-15. The spectrum shows the double horn feature expected of spiral galaxies viewed at a nearly edge-on inclination. We believe it is possible that the high velocity cloud (HVC), HVC $280.8+09.2+223$, identified in the HIPASS HVC catalogue (Putman et al., 2002) which has a velocity resolution of $26 \mathrm{~km} \mathrm{~s}^{-1}$, could instead be emission from this edge-on galaxy, ESO 214-15. The position of HVC $280.8+09.2+223$ is only $2^{\prime}$ from the center of ESO $214-15$, well within the beam size of Parkes, and is listed with a local standard of rest velocity of $V_{L S R}=223 \mathrm{~km} \mathrm{~s}^{-1}$ with a full width at half-maximum (FWHM) of $48 \mathrm{~km} \mathrm{~s}^{-1}$, comparable to our converted $V_{L S R}=240 \mathrm{~km} \mathrm{~s}^{-1}$ and our linewidth value at $50 \%$ maximum of $W_{50}=42 \mathrm{~km} \mathrm{~s}^{-1}$. The similarities in the velocities and linewidths, along with the nearly identical position, and optical alignment with the peak of the H I, lead us to believe that this is emission from ESO 214-15, rather than an HVC. We used the Masters et al. (2014) K band Tully-Fisher relation to calculate a distance of $2.7 \mathrm{Mpc}$, well outside the ESO 214-17 group, with an absolute K-band magnitude of -14.9, which enabled us to derive other H I parameters listed in Table 2.5.

Positions, along with measured and derived galaxy properties for all confirmed H I sources found in our data cube are listed in Table 2.5.

\subsection{Discussion}

Here we will consider the possibilities for the origin and future of the low column density bridge between ESO 214-17 and ESO 214-18. 
The bridge between these two galaxies is indicative of a larger picture of interaction between the two galaxies. In order to understand what is happening in this system, we can look at the morphology of the central host galaxy, ESO 214-17. The spiral arms of ESO 214-17 lie at a large pitch angle, a possible suggestion about the origin of this galaxy. A grand-design spiral galaxy with loose spiral arms such as this one suggests either an orbit of a companion or multiple passages by that companion (D'Onghia et al., 2016; Dobbs et al., 2010; Oh et al., 2015). As such, the bridge we are seeing may be a relic of one of these passages. However, since we do not detect a tail which is a common result of past interaction, we could potentially be seeing the beginnings of interaction between these galaxies.

Additionally, if this bridge indicates that the galaxy is undergoing current or recent interaction, enhanced star formation should be detected in the companion, ESO 214-18, and the host galaxy (Salo \& Laurikainen, 1993; Bernard et al., 2012; Semczuk et al., 2018). Merging galaxies have been shown to undergo star formation rate enhancement, and simulations have shown that roughly half of the star formation activity in the local Universe is triggered by minor-mergers (Larson \& Tinsley, 1978; Kaviraj, 2014). To search for this interaction-enhanced star formation, a combination of $\mathrm{UV}+\mathrm{IR}$ observations would be ideal to trace the star formation of young stars as UV traces star formation on short timescales, while IR traces the dust emission where UV becomes obscured by dust.

We can look to the gas fraction and the mass ratio to tell us about the past and future of this system. The gas fraction and mass ratio of the two galaxies can also have an effect on the interaction history and potential merger morphology (Lotz 
et al., 2010). Specifically, high baryonic gas fractions (they use $f_{\text {gas }}>53 \%$ in the simulation) produced more disc star formation, and remained asymmetric for $\sim 1-1.4$ Gyr, making it equally likely to be detected if it were either a major or minor merger. For comparison, the neutral gas fraction of ESO 214-17 is estimated to be $f_{H I}=51 \%$, and would be solidly classified as a minor merger. Scudder et al. (2012) show that star formation enhancement effected by the mass ratio can be seen out to at least 80 kpc in projected distance. The projected distance of ESO 214-18 from ESO 214-17 is $38.6 \mathrm{kpc}$, well within this limit so we would expect to see star formation enhancement in this system.

As this is a minor merger, we expect a low interaction strength, which can be quantified with the Dahari parameter, $Q$. This parameter estimates the gravitational interaction strength by measuring the tidal strength a galaxy experiences by a companion galaxy. Strong interactions are defined above a $Q$ value of one (Dahari, 1984), with

$$
Q=\frac{M_{\text {Comp }}}{M_{\text {Central }}} \times\left(\frac{R_{\text {Sep }}}{R_{\text {Central }}}\right)^{-3}
$$

Here, $M_{C o m p}$ is the dynamical mass of the companion galaxy, and $M_{\text {Central }}$ is the dynamical mass of the central galaxy, both in units of $M_{\odot} \cdot R_{\text {Sep }}$ is the physical separation between the centers of the two galaxies, and $R_{\text {Central }}$ is the radius of the central galaxy, both in kpc. This gives of a $Q$ value of 0.036 , indicating some minor interaction.

We also calculated the maximum extent of the central/companion system, or 
the Jacobi radius, as

$$
r_{\mathrm{J}}=R_{\text {Sep }}\left(\frac{M_{\text {Comp }}}{3 M_{\text {Central }}}\right)^{1 / 3}
$$

The estimation for this radius $\left(r_{\mathrm{J}} \approx 20 \mathrm{kpc}\right)$ is smaller than the projected separation of the two galaxies, indicating that the interaction is weak, or that the system is in the beginning stages of interaction.

If we test the stability of the orbit, we can determine the time-scale in which the system will merge. Once a satellite enters the dark matter halo of a galaxy, it experiences a dynamical friction force, pulling it toward the center of the galaxy until it merges after some time. The time-scale from when the satellite enters the halo until merger is called the dynamical friction time-scale (Binney \& Tremaine, 2008), which is calculated as

$$
t_{\text {fric }}=\frac{1.17 v_{c} R_{S e p}^{2}}{\ln (\Lambda) G M_{C o m p}}
$$

The dynamical friction time-scale equation uses the circular velocity $v_{c}$, the physical separation between the two galaxies $R_{\text {Sep }}$, the gravitational constant $G$, and the dynamical mass of the companion galaxy $M_{C o m p}$. The Coulomb logarithm, $\ln (\Lambda)$, which describes the effectiveness of an encounter given the impact parameters, is given as

$$
\ln (\Lambda)=\ln \left(\frac{R_{S e p} v_{c}^{2}}{G M_{C o m p}}\right)
$$

In the ESO 214-17/18 system, we do not know the circular velocity so instead, we take the difference in systemic velocities of the two galaxies, similar to Pisano \& 
Wilcots $(2000)$. Our resulting $t_{\text {fric }} \approx 4$ Gyr tells us that this companion will merge with ESO 214-17 within this relatively short timescale.

Since we do have a map of the entire virial volume of ESO 214-17, we can compare the number of satellite galaxies we detected within this volume with predictions of the number of satellites expected to be found within the virial radius of a galaxy of a certain stellar mass. Although we are only looking at the HI and not other wavelengths, we will compare our results with those of Sales et al. (2013) and Guo et al. (2011). Sales et al. (2013) determine the average number of satellites per host as a function of the difference in r-band absolute magnitudes $\left(\Delta M=M_{r}^{s a t}-M_{r}^{p r i}\right)$, where $\Delta M$ is plotted between 0 and 5 . These satellite numbers depend on the stellar mass of the primary, $M_{*}^{\text {pri }}$, and of note is the lack of dependence on stellar masses below $M_{*}^{\text {pri }}=10^{10} M_{\odot}$. With a stellar mass of $M_{*}=10^{8.92} M_{\odot}$, derived from the Bell \& de Jong (2001) relation, ESO 214-17 solidly falls into this category. For absolute magnitude differences between 0 and 5, the prediction of the number of satellites would be between 0.002 and 0.3 . We were not able to obtain r-band magnitudes for both ESO 214-17 and ESO 214-18, so we cannot say for certain where along those values the number of satellites should fall. However, since we only need a difference in the absolute magnitudes, we substitute r-band for K-band magnitudes ${ }^{8}$ as this is the information available for these two galaxies. We use the same distance of $13.1 \mathrm{Mpc}$ for both, and we get absolute K-band magnitudes of $M_{K}^{\text {sat }}=-17.46$ and $M_{K}^{p r i}=-19.37$. With $\Delta M=1.9$, the predicted number of satellites at this absolute magnitude is $0.04-0.05$ satellites.

Guo et al. (2011) use the ratio of satellite stellar mass to primary stellar mass 
to determine the average number of satellites around a primary galaxy of a certain stellar mass. They too found a lack of dependence on stellar mass below $M_{*}^{\text {pri }}=$ $10^{10} M_{\odot}$. The range of satellite counts per host for a primary galaxy of stellar mass $M_{*}=10^{8.92} M_{\odot}$ is shown to be between 0.006 and 1 . Once again, we do not have a stellar mass for ESO 214-18, but if we assume that the ratio of satellite to primary stellar masses would be similar to the ratio of satellite to primary H I masses, we can simply substitute stellar masses for H I masses and take the ratio. If $M_{*}^{\text {sat }} / M_{*}^{\text {pri }} \propto$ $M_{\mathrm{HI}}^{\text {sat }} / M_{\mathrm{HI}}^{\text {pri }}$, then this ratio becomes 0.1 . Using this ratio and our primary stellar mass of $M_{*}=10^{8.92} M_{\odot}$, the number of satellites predicted is also between $0.04-0.05$, in good agreement with Sales et al. (2013). While we do not have the exact data to perfectly estimate the number of predicted satellite galaxies around a primary galaxy, using the tools at hand, we can compare what we detect with these predictions. In the case of primary galaxy, ESO 214-17, we obviously detect exactly one satellite, ESO 214-18. Predictions of the number of satellites around a galaxy like ESO 214-17 is less than one, therefore these models are underpredicting the number of satellites within the primary galaxy's virial volume.

\subsection{Conclusions and Future Work}

We present the first results from the IMAGINE survey with the Parkes Radio Telescope. We have detected the 21-cm line of neutral hydrogen connecting two nearby spiral galaxies, ESO 214-17 and ESO 214-18, in velocity space, revealing both

\footnotetext{
${ }^{8}$ http://simbad.u-strasbg.fr/simbad/ lists K-band magnitudes for ESO 214-17 as 11.212, and ESO $214-18$ as 13.128
} 
a kinematic and a likely spatial interaction. These observations go deeper than other H I surveys of nearby galaxies outside of the Local Group, and reach column density sensitivities of $N_{\mathrm{HI}}=1.3 \times 10^{16} \mathrm{~cm}^{-2}$ per $2.6 \mathrm{~km} \mathrm{~s}^{-1}$ channel. We were able to reach these sensitivities throughout the viral volume of ESO 214-17, detecting several other sources in H I. In reaching these column density sensitivities, the Parkes-IMAGINE survey will quantify the amount of $\mathrm{HI}$ in the CGM of nearby spiral galaxies. A summary of our results are given here:

1. Our deep observations of the H I within the virial volume of ESO 214-17 revealed a continuous connection to a companion galaxy, ESO 214-18. This bridge peaks at a $20 \sigma$ level of $N_{\mathrm{HI}}=2.7 \times 10^{17} \mathrm{~cm}^{-2}$ per $2.6 \mathrm{~km} \mathrm{~s}^{-1}$ channel.

2. In addition to the central galaxy, we detected the spiral galaxies ESO214-18, ESO 264-35, and made a detection with possibly the first velocity information for ESO 214-15.

3. We calculated the dynamical friction timescale, the time it takes a satellite to merge with the primary once it has entered the dark matter halo. This timescale, $t_{\text {fric }} \approx 4 \mathrm{Gyr}$, is relatively short and the satellite will therefore merge with the primary in this amount of time.

4. We compared the number of detected satellite galaxies within the virial radius of ESO 214-17 with model predictions. We concluded that in this one example, the models are underpredicting the number of satellites that should be within the virial volume of our primary galaxy. 
We will continue our investigation of the neutral atomic gas of the remainder of the Parkes-IMAGINE sample of 28 nearby galaxies in Chapter 4, where we will be able to describe the statistical and global properties of the CGM surrounding these galaxies as seen through our deep, sensitive observations. With the full Parkes-IMAGINE sample, we will be able to state the amount of low column density H I seen in spiral galaxies in the nearby universe among a range of masses and environments. We will also be following up with the ATCA observations of ESO 214-17 to look for spatially resolved H I between ESO 214-17 and ESO 214-18. The higher resolution data from ATCA-IMAGINE will provide us with column density thresholds for smaller regions of the galaxy which could then be compared to star forming regions, where high $\mathrm{H} \mathrm{I}$ column densities are generally associated with regions of star formation. With this information, we will be able to look for recent enhancement of star formation, which would be another indication of interaction between ESO 214-17 and ESO 214-18. 


\section{Chapter 3}

\section{Analysis of the Diffuse H I Environment Around 18 MHONGOOSE}

\section{Galaxies}

\subsection{Abstract}

We present the analysis of the diffuse, low column density HI environment of 18 MHONGOOSE galaxies. We obtained deep observations with the Robert C. Byrd Green Bank Telescope, and reached $1 \sigma$ column density sensitivities of $N_{\mathrm{HI}}=$ $5.6 \times 10^{16} \mathrm{~cm}^{-2}$ per $\sim 6 \mathrm{~km} \mathrm{~s}^{-1}$ channel. We analyze the environment around these galaxies, with a focus on H I gas that reaches column densities below $N_{\mathrm{HI}}=10^{19} \mathrm{~cm}^{-2}$. Our environmental analysis allows us to calculate the total amount of H I gas around the galaxies revealing that six of these galaxies contained excess H I outside of their disks. We quantify the diffuse amount of gas in the galaxy with a diffuse neutral fraction, $f_{19}$, which is the ratio of $\mathrm{HI}$ mass below $10^{19} \mathrm{~cm}^{-2}$ and the total measured $\mathrm{H} \mathrm{I}$ mass of the galaxy. We found the range of diffuse neutral gas percentages fell between $5-93 \%$. We also compare this diffuse fraction, $f_{19}$, with a variety of environmental properties, and find a correlation with several properties supported by cold mode accretion models, in particular with baryonic mass, galaxy density, and rotational velocity. 


\subsection{Introduction}

The question of how galaxies get their gas and how they use this gas to continue to form stars remains among the main unanswered questions in astronomy. We know that as the star formation rate density over cosmic time has decreased since a redshift of $\mathrm{z} \sim 2$ (Madau \& Dickinson, 2014), the neutral hydrogen gas density has remained nearly constant since z $\sim 4$ (Rhee et al., 2018). Di Teodoro \& Fraternali (2014) show that mergers of dwarf galaxies with spiral galaxies do not provide sufficient extra mass to sustain star formation in those galaxies. Even so, if star formation in spiral galaxies is not sustained by mergers alone, then they must be accreting the gas from the intergalactic medium (IGM).

Gas accreting from the IGM flows into the galaxy via cold, diffuse filamentary structures (Birnboim \& Dekel, 2003; Katz et al., 2003; Kereš et al., 2005). The gas travels from the cosmic web (Bond et al., 1996) through these filaments, and into the circumgalactic medium (CGM) of galaxies before falling onto the galaxy disk. The process of accretion described here is termed cold-mode accretion (Kereš et al., 2005). Observations of the CGM and disks of galaxies will provide insight into cold accretion and potentially direct detections of this gas falling onto galaxies.

Deep HI surveys provide the observational link to direct detections of the effects of cold mode accretion from the IGM. The MeerKAT H I Observations of Nearby Galactic Objects; Observing Southern Emitters (MHONGOOSE; de Blok et al. (2016)) survey will provide high spatial resolution, high column density sensitivity maps of 30 nearby disk and dwarf galaxies. Most other H I surveys achieve 
one or the other, but not both, with the exception of IMAGINE with ATCA, which still has a much lower angular resolution than MHONGOOSE. HIPASS (Koribalski et al., 2004) and ALFALFA (Haynes et al., 2011) surveys provide an extremely large number of HI detections, although neither at high resolution or with column density sensitivities matching ours. High resolution surveys such as HALOGAS (Heald et al., 2011) or THINGS (Walter et al., 2008) achieve column density sensitivities of $\sim 10^{19} \mathrm{~cm}^{-2}$ and $\sim 10^{20} \mathrm{~cm}^{-2}$, which is too high to detect diffuse gas from the IGM. The MHONGOOSE survey will use the South African MeerKAT radio telescope, a 64-dish precursor to the Square Kilometre Array (SKA), to map these 30 galaxies to a $3 \sigma$ column density detection limit of $7.5 \times 10^{18} \mathrm{~cm}^{-2}$ over a $16 \mathrm{~km} \mathrm{~s}^{-1}$ linewidth at an angular resolution of $30^{\prime \prime}$. At their poorest angular resolution, 90", this column density limit goes down to $5.5 \times 10^{17} \mathrm{~cm}^{-2}$. The high resolution maps produced by MHONGOOSE will further our understanding of how galaxies get their gas, how galaxies sustain star formation, and how matter that we can detect relates to the dark matter associated with galaxies, influencing galactic evolution. Specifically, this survey will help us to understand how gas flows in or out of galaxies, the conditions that allow the fuelling of star formation, accretion from the IGM, and ultimately its connection to the cosmic web.

Early observations of a subset of MHONGOOSE galaxies were presented in Sorgho et al. (2019c, hereafter S19), using MeerKAT, KAT-7, the seven-dish MeerKAT precursor array, and the Robert C. Byrd Green Bank Telescope (GBT). The subset of galaxies mapped with the GBT are presented here, with the description of the MHONGOOSE sample in Section 3.3. We describe our GBT observations and data 
reduction process in Section 3.4. Two galaxies not presented in S19 are presented in Section 3.5. The analysis we performed on all of these galaxies is described in Section 3.6. Our results are presented in Section 3.7. We discuss these results in Section 3.8 and summarize our findings in Section 3.9.

\subsection{MHONGOOSE Galaxy Sample}

The MHONGOOSE sample of galaxies was chosen from galaxies having been previously detected in multiple wavelengths (H I from HIPASS, $\mathrm{H} \alpha$, optical, infrared,

and ultraviolet from the SINGG and SUNGG (Meurer et al., 2006) surveys). An equal number of galaxies were selected for each of six mass bins within a range of $6<\log _{10}\left(\mathrm{M}_{\mathrm{HI}}\right)<11 M_{\odot}$ for a total of 30 galaxies with inclinations of edge-on, faceon, and between $50^{\circ}-60^{\circ}$, and a wide range of morphologies from dwarf irregulars to grand spirals. The sample of galaxies chosen for this set of observations coincides with the galaxies from the MHONGOOSE sample $\left(\delta<10^{\circ}\right)$ which can be seen by the GBT, which has a lower limit of declinations $\delta>-46^{\circ}$. This narrows the sample to 18 galaxies. GBT integrated intensity (moment 0) maps and global H I profiles for 16 of these sources were presented in S19, with two others, NGC 1744 and NGC 7424, having been observed separately and introduced here.

Each galaxy in the sample presented here, as well as the full MHONGOOSE sample, has a Galactic latitude of $|b|>30^{\circ}$, peak H I fluxes, as detected in HIPASS, of greater than $50 \mathrm{mJy}$, and Galactic standard of rest velocities $>200 \mathrm{~km} \mathrm{~s}^{-1}$. Additionally, each source is below a declination of $\delta<10^{\circ}$ and is within a distance of $30 \mathrm{Mpc}$. 


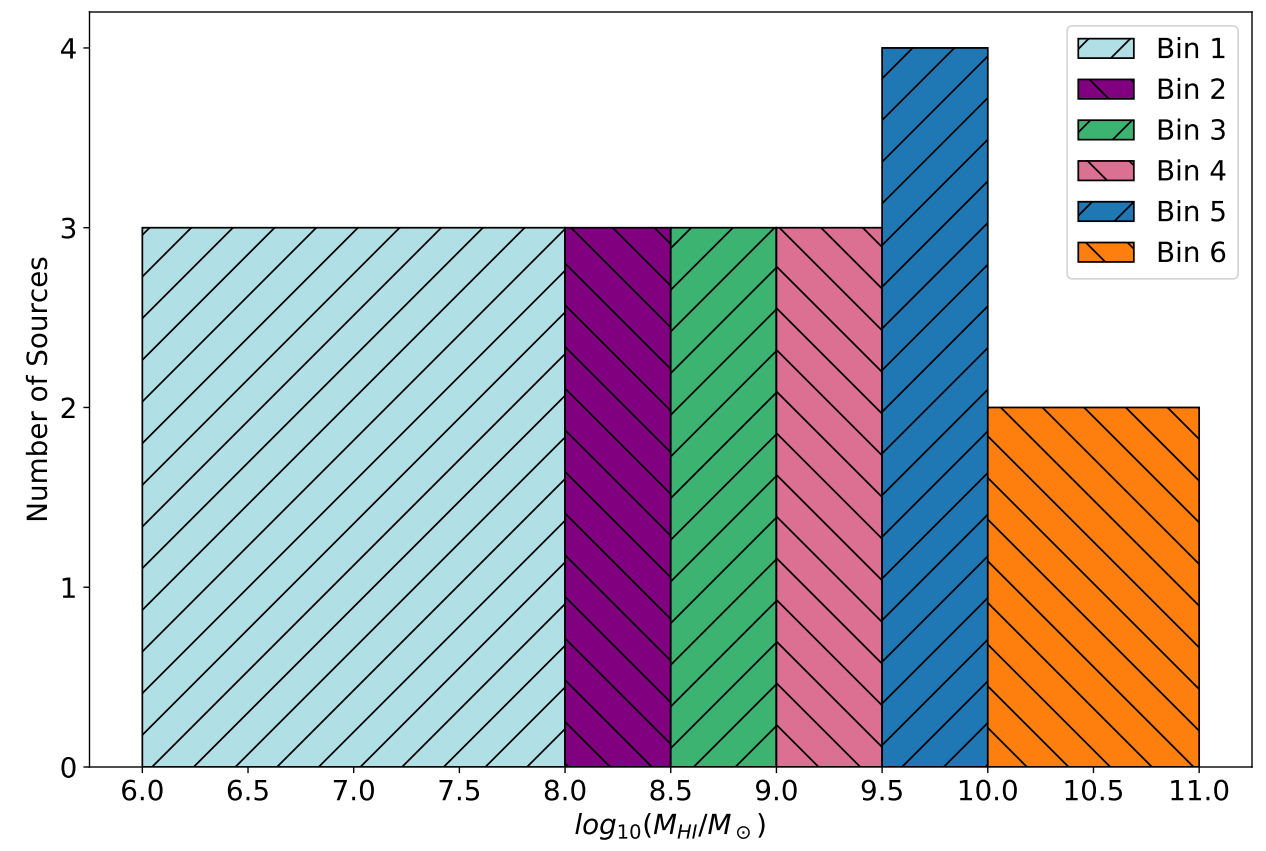

Figure 3.1: Histogram of H I masses in our MHONGOOSE sample. Each bin is separated by color and hatch marking. This histogram demonstrates our fairly uniform coverage of the H I mass range in the full MHONGOOSE sample.

The MHONGOOSE sample was chosen to uniformly cover the range of $M_{\mathrm{HI}}$ listed above, and our GBT sample covers that range nearly uniformly as well. Coverage of the H I mass range in each bin can be seen in Figure 3.1.

\subsection{Observations and Data Reduction}

Observations for the 16 galaxies presented in S19 were carried out between August 2016 and January 2017 for project GBT16B-212. Each of these galaxies were observed for 10 hours each, with a total of 160 hours, with a theoretical noise sensitivity of $15 \mathrm{mK}$ over $5.2 \mathrm{~km} \mathrm{~s}^{-1}$ channels. This noise sensitivity corresponds to a column density sensitivity of $N_{\mathrm{HI}} \sim 10^{18} \mathrm{~cm}^{-2}$ over the same channel width. The 
two additional galaxies presented here (NGC 1744 and NGC 7424) were observed over the course of three GBT observing semesters (GBT15B-346, GBT16B-408, and GBT17A-478) from 2015 to 2017, for a total of 20 hours on NGC 1744 and 14 hours on NGC 7424. We observed these two galaxies over a bandwidth of $23.4 \mathrm{MHz}$, with a frequency resolution of $0.715 \mathrm{kHz}$. We used the sources $3 \mathrm{C} 48$ and $3 \mathrm{C} 147$, which have stable, well-understood fluxes, over the course of 12 observing nights to calibrate our data. We smoothed the data using a boxcar function with a final smoothed velocity resolution of $5.1 \mathrm{~km} \mathrm{~s}^{-1}$, or $24.3 \mathrm{kHz}$. The data was fitted with either a $3^{\text {rd }}$ or $4^{\text {th }}$ order polynomial after baseline subtraction. We flagged all of the data manually, and gridded all of the data into data cubes with two spatial axes (RA and Dec.) and one velocity axis.

For each of our 18 galaxies, we used the GBT's VErsatile GBT Astronomical Spectrometer (VEGAS) backend in L-band (1.15-1.73 GHz), where the FWHM beamwidth is $9.1^{\prime}$, to map $2^{\circ} \times 2^{\circ}$ regions around each source. Data cubes were made for all 18 sources with pixel sizes of $1.75^{\prime}$. The 16 sources from S19 were smoothed to a velocity resolution of $6.4 \mathrm{~km} \mathrm{~s}^{-1}$. Further details regarding the data reduction techniques of these 16 galaxies are given in S19.

We reached rms noise levels of 3.2 to 10.4 mJy in the cubes, corresponding to a $1 \sigma$ column density sensitivity of $N_{\mathrm{HI}}=1.02 \times 10^{17} \mathrm{~cm}^{-2}$ per $5.1 \mathrm{~km} \mathrm{~s}^{-1}$ channel. At the noise level reached in this cube, a detectable signal at the $5 \sigma$ level over a $20 \mathrm{~km} \mathrm{~s}^{-1}$ linewidth is $N_{\mathrm{HI}}=2 \times 10^{18} \mathrm{~cm}^{-2}$. Our $1 \sigma$ mass sensitivities extend down to $1.6 \times 10^{5}$ $M_{\odot}$ 


\subsection{Properties of galaxies}

Data cubes and integrated intensity (moment 0 ) maps for the 16 sources mentioned above were inspected and searched for anomalous H I in S19. In this section we will present the two additional sources, NGC 1744 and NGC 7424, and discuss their properties. These properties are listed in Table 3.2.

\subsubsection{NGC 1744}

NGC 1744 is an inclined $\left(69.9^{\circ}\right) \mathrm{SBcd}^{1}$ galaxy, or a patchy-armed barred spiral without a bulge. It is one of the more massive galaxies in our sample, which we measured the the total HI mass to be $7.6 \times 10^{9} M_{\odot}\left( \pm 2.1 \times 10^{8}\right)$. We measured a total integrated flux of $174.7 \mathrm{Jy} \mathrm{km} / \mathrm{s}$ with an rms noise of $\sigma_{r m s}=3.2 \mathrm{mJy}$. We measured the full width at $20 \%$ maximum of the velocity profile to be $207.2 \mathrm{~km} / \mathrm{s}$ with a systemic velocity of $743 \mathrm{~km} / \mathrm{s}$, so we calculated the dynamical mass of NGC 1744 as $3.6 \times 10^{10} M_{\odot}$. These and other properties of NGC 1744 were tabulated in Table 3.2 .

We detected one additional source in the NGC 1744 data cube. Sbc ${ }^{1}$ galaxy, ESO 486-G021 (Figure 3.5), is detected with an integrated H I flux density of 24.5 Jy $\mathrm{km} \mathrm{s}^{-1}$. The systemic velocities measured from the two galaxies differ by $\sim$ $100 \mathrm{~km} \mathrm{~s}^{-1}$, and while this is similar enough to suspect interaction, their angular separation is $58^{\prime}$. At a distance of $12 \mathrm{Mpc}$, that becomes a physical separation of $\sim 200 \mathrm{kpc}$, and with a calculated Jacobi radius (Eq. 2.7) of $\sim 79 \mathrm{kpc}$ with the dy-

\footnotetext{
${ }^{1}$ http://leda.univ-lyon1.fr
} 


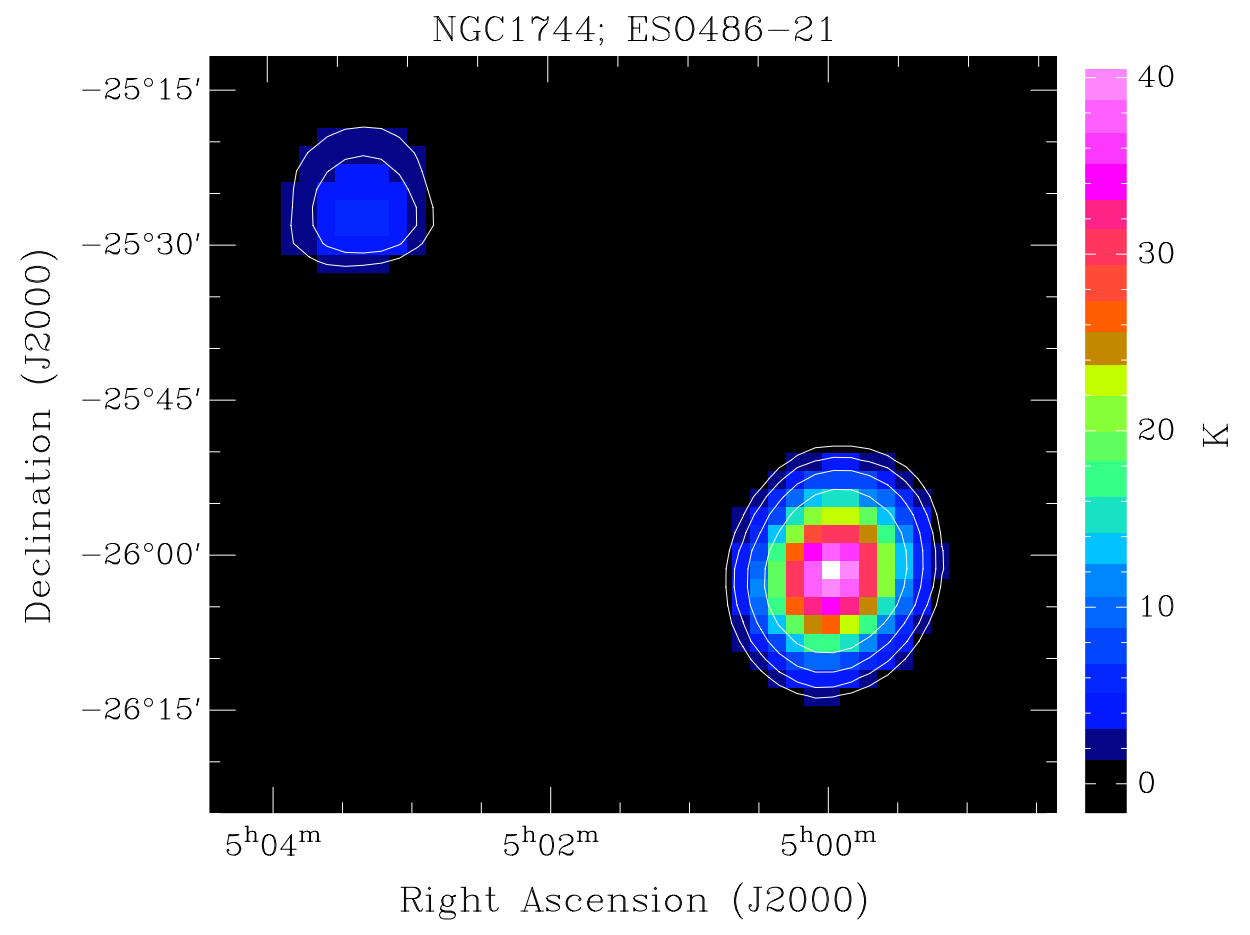

Figure 3.2: Integrated intensity (moment 0) map of NGC 1744 with companion, ESO486-21 in upper left. Contours at 10, 20, and 40 times the integrated rms noise of $0.15 \mathrm{~K}$. The column density equivalent to these contour levels is $N_{\mathrm{HI}}=0.69,1.3$, 2.7 , and $5.5 \times 10^{19} \mathrm{~cm}^{-2}$.

namical mass of the companion at $2.2 \times 10^{9} M_{\odot}$, this becomes a distance too large to consider plausible interaction. Further, we do not detect any extraplanar or anomalous H I around NGC 1744 at these levels. A total H I intensity map including both NGC 1744 and ESO 486-G021 can be seen in Figure 3.2. Spectra of NGC 1744 can be seen in Figure 3.4. At a mass sensitivity in this cube of $7.2 \times 10^{5} M_{\odot}$, and because we did not detect any anomalous H I, we can say that there are no additional sources with $3 \sigma$ H I mass greater than $7.2 \times 10^{5} M_{\odot}$, apart from ESO 486-G021. 


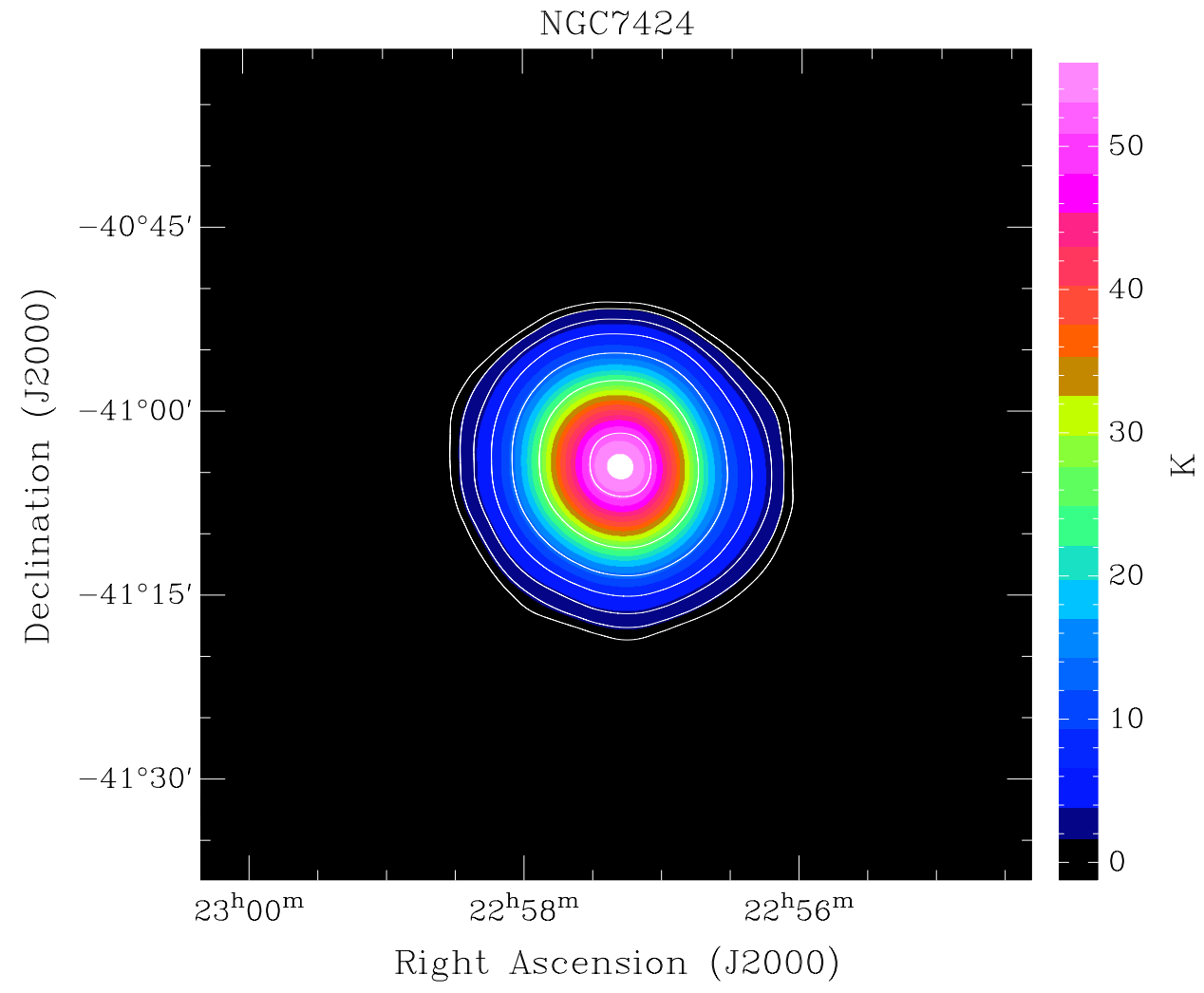

Figure 3.3: Integrated intensity (moment 0) map of NGC 7424. Contours at 5, 10, 20, 40, 80, and 160 times the integrated rms noise of $0.16 \mathrm{~K}$. The column density equivalent to these contour levels is $N_{\mathrm{HI}}=0.7,1.48,2.9,5.9$, and $11.9 \times 10^{19} \mathrm{~cm}^{-2}$. 

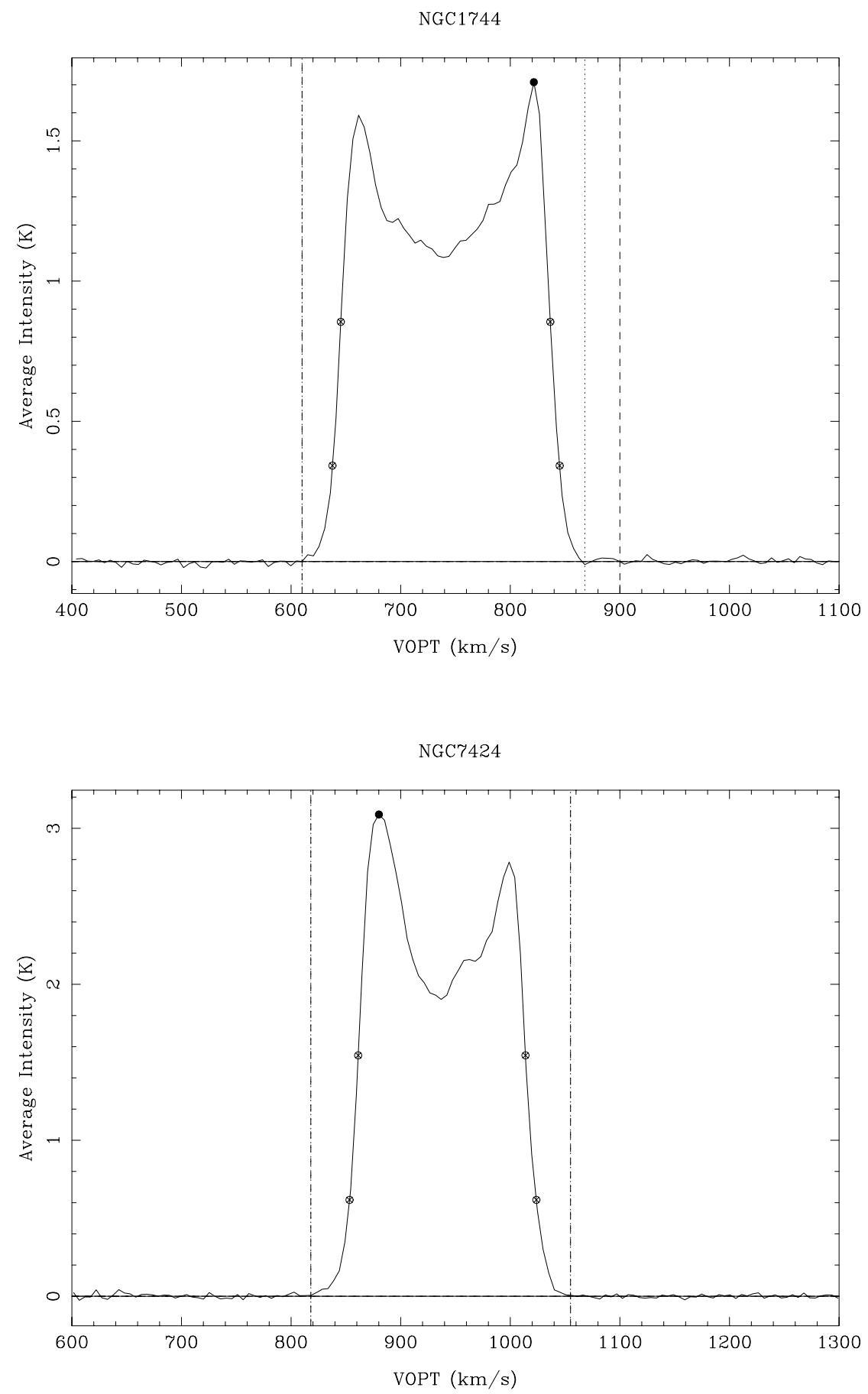

Figure 3.4: Total integrated H I profiles of NGC 1744 (upper panel) and NGC 7424. Measurements are taken between the dashed and dotted vertical lines. The open circles and $\times$ 's indicate the $20 \%$ and $50 \%$ FWHM velocity widths. The filled circle represents the peak value of the specified profile. Masking is applied between the dotted lines. 


\subsubsection{NGC 7424}

NGC 7424 is a mostly face-on SABcd (intermediate strength barred spiral, patchy arms, lacking a bulge) galaxy (de Vaucouleurs et al., 1991), and one of the most H I massive sources in the survey. It falls into the mass bin: $10<\log _{10}\left(\mathrm{M}_{\mathrm{HI}}\right)<11$ $M_{\odot}$, one of the more unexplored mass regimes in low column density H I studies. Measurements of these massive galaxies are crucial as this mass regime contains galaxies with a large enough gravitational potential to pull gas from the intrafilament medium, utilizing cold mode accretion and replenishing their supply of cold gas (Kleiner et al., 2017). We measured a total H I mass of $2.4 \times 10^{10} M_{\odot}\left( \pm 6.2 \times 10^{8}\right)$ in NGC 7424 , where the mass sensitivity in the cube is $2.3 \times 10^{6} M_{\odot}$ corresponding to a $1 \sigma$ column density sensitivity of $N_{\mathrm{HI}}=9.7 \times 10^{16} \mathrm{~cm}^{-2}$ per $5.1 \mathrm{~km} \mathrm{~s}^{-1}$ channel. NGC 7424's global H I profile and moment map can be seen in Figure 3.4.

We detected two additional galaxies within our map of NGC 7424: the Sd galaxy ESO 346-G018 with an angular separation of 58', and the SBbc galaxy NGC 7462 with an angular separation from NGC 7424 of $64^{\prime}$. However, due to their positions at the edges of our cube, we were not able to confidently make measurements of the H I. Nevertheless, the total integrated profiles were created of each and can be seen in Figure 3.5. No other significant detections of H I were seen throughout the cube.

\subsubsection{Discussion of the Satellites around NGC 1744 and NGC 7424}

The lack of satellites detected within the virial radius of these galaxies is potentially consistent with the 'Missing Satellites Problem' (Klypin et al., 1999; Moore 

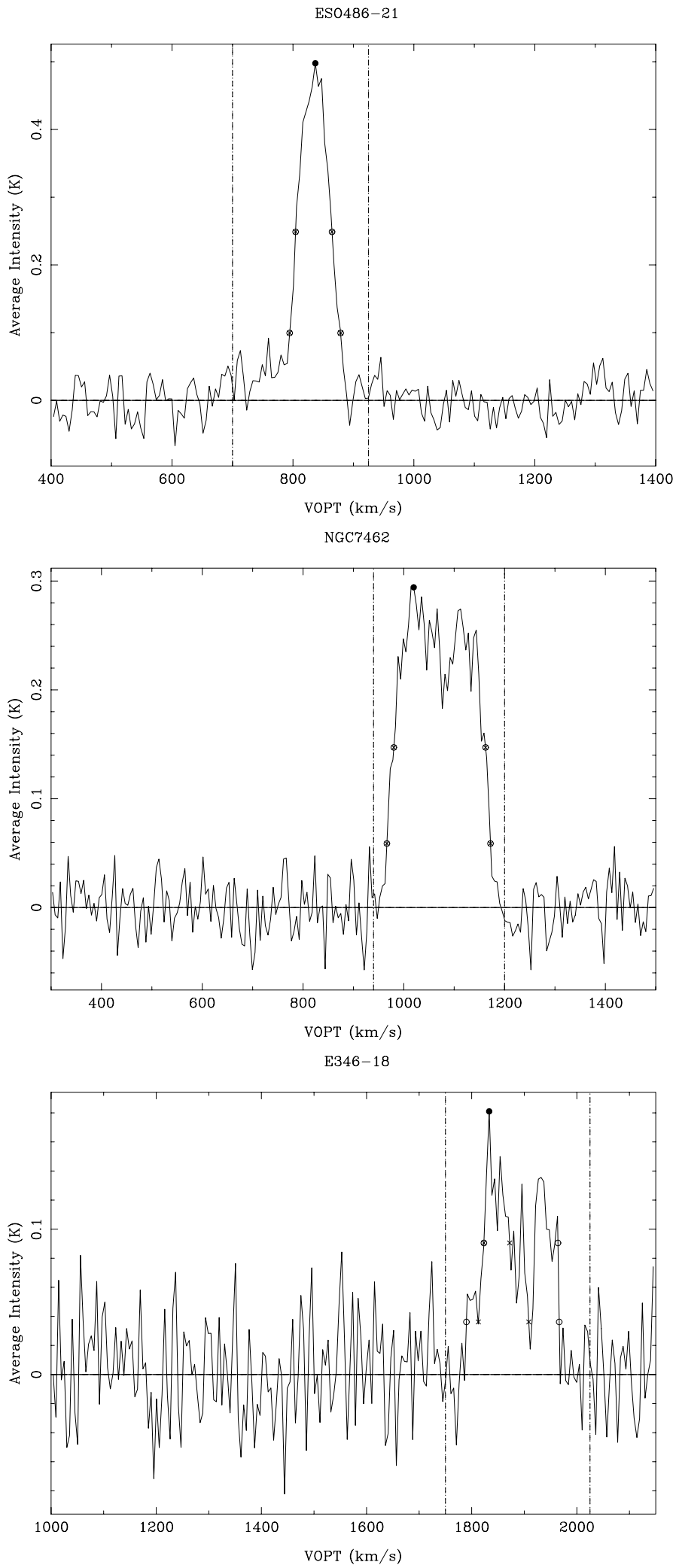

Figure 3.5: Same as Figure 3.4, but for ESO486-21 (top), NGC7462 (center), and ESO346-18 (lower). 
et al., 1999) in which more dark matter halos are predicted to exist around galaxies such as the Milky Way than have been detected through observations, with the caveat that when looking at $\mathrm{H}$ I we would almost certainly miss satellites that lack H I, such as dwarf spheroidal galaxies. Also potentially consistent with what we have found is the solution to this problem from Kim et al. (2017). They demonstrate that applying completeness corrections to the Sloan Digital Sky Survey, with a detection efficiency of satellites with luminosities $L>340 L_{\odot}$, gives the observed number of satellites predicted by a cold dark matter model. Finding 1-2 luminous satellites around galaxies the sizes of NGC 1744 and NGC 7424 is on the order of the findings by Kim et al. (2017).

\subsection{Analysis}

Each source in this survey was analyzed by extracting information from the data cubes and deriving individual galaxy properties, followed by creating both masked and unmasked total integrated intensity (moment 0) maps, and measuring the global properties of the galaxy environments from these images. Moment 0 maps for each source can be found in S19. In doing this, we can identify the statistical properties over a wide population of galaxies.

\subsubsection{Total integrated $\mathrm{H}$ i flux profiles}

We created total integrated H I flux profiles for each galaxy using the MIRIAD task MBSPECT. MBSPECT makes a measurement of the data cube over a wide velocity 
range, where channels including emission are masked, and a first-order polynomial is fitted to the baseline, removing residual baseline variation. We obtain the $1 \sigma \mathrm{rms}$ noise over emission-free channel ranges, as well as the total integrated flux, and the linewidths of each source at 20\% (W20) and 50\% (W50) maximum. These measured values are listed in Table 3.2. Each galaxy's global line profile can be seen in Figures 3.15 through 3.17 .

\subsubsection{Derived galaxy properties}

For each data cube, we calculate the per channel $1 \sigma$ column density sensitivity. We use measurements from MBSPECT to derive physical properties of each galaxy in the sample, such as the total H I masses, total dynamical masses, and neutral gas fractions.

Column densities are calculated with

$$
N_{\mathrm{HI}}=1.822 \times 10^{18}\left(\frac{T_{\mathrm{B}}}{\mathrm{K}}\right)\left(\frac{d v}{\mathrm{~km} \mathrm{~s}^{-1}}\right) \mathrm{cm}^{-2},
$$

where $T_{\mathrm{B}}$ is the brightness temperature in units of Kelvin, and $d v$ is the resolution of our data in $\mathrm{km} \mathrm{s}^{-1}$. When estimating the detectable column density level in the cube, we use a $5 \sigma$ limit and a minimum linewidth of a typical H I detection $\left(20 \mathrm{~km} \mathrm{~s}^{-1}\right)$.

We integrate over the total flux values to estimate the H I mass of each source, assuming the $\mathrm{H} \mathrm{I}$ is optically thin, using:

$$
M_{\mathrm{HI}}=2.36 \times 10^{5} D^{2} \int_{v_{1}}^{v_{2}} S(v) d v \mathrm{M}_{\odot} .
$$


Here, the distance, $D$, is in $\mathrm{Mpc}$, and $\int_{v_{1}}^{v_{2}} S(v) d v$ is the total integrated flux over velocities enclosing the $\mathrm{H}$ I profile $v_{1}$ to $v_{2}$, and is in units of $\mathrm{Jy}_{\mathrm{km} \mathrm{s}}{ }^{-1}$. The brightness temperatures from each cube were divided by the $1.86 \mathrm{~K} \mathrm{Jy}^{-1}$ gain of the GBT to obtain units of Jy. We calculated this gain using an aperture efficiency of $\sim 0.65$ (Boothroyd et al., 2011) for the GBT at $1420 \mathrm{MHz}$.

In order to calculate the total dynamical mass of each galaxy, we first calculated the physical H I diameter, $D_{\mathrm{HI}}$, using the Broeils \& Rhee (1997) scaling relation (Eq. $2.5)$, which is dependent on the optical diameter of the galaxy, $D_{25}$, measured at the $25^{\text {th }}$ mag $\operatorname{arcsec}^{-2}$ isophote in the B-band. We used values for $D_{25}$ from the LyonMeudon Extragalactic Database (LEDA). We use the dynamical mass equation:

$$
M_{\mathrm{dyn}}=2.3 \times 10^{5}\left(\frac{v_{\text {rot }} / \sin (i)}{\mathrm{km} \mathrm{s}^{-1}}\right)^{2}\left(\frac{r}{\mathrm{kpc}}\right) \mathrm{M}_{\odot}
$$

where $v_{\text {rot }}$ is the rotation velocity in $\mathrm{km} \mathrm{s}^{-1}$ corrected for inclination, $i$, also taken from LEDA. These properties obtained fron NED and LEDA can be seen in Table 3.1. We use our linewidth at $20 \%$ maximum to estimate $v_{\text {rot }}$ as $v_{\text {rot }}=W_{20} / 2$. Dynamical masses are calculated inside the $\mathrm{H}$ I radius, $r$ in kpc, calculated as $D_{\mathrm{HI}} / 2$.

The last property we derive using the information at hand, is the neutral H I fraction. This is defined as the fraction of H I mass in the galaxy and is calculated with $f_{\mathrm{HI}}=M_{\mathrm{HI}} / M_{\mathrm{dyn}}$. Each of these derived properties are listed in Table 3.2 for every galaxy in the sample. 
Table 3.1: Sample of MHONGOOSE Galaxies

\begin{tabular}{|c|c|c|c|c|c|}
\hline Source & $\begin{array}{c}\text { R.A. }(2000.0) \\
\text { [deg] } \\
(2)\end{array}$ & $\begin{array}{c}\text { Decl. }(2000.0) \\
\text { [deg] } \\
(3)\end{array}$ & $\begin{array}{c}\mathrm{D} \\
{[\mathrm{Mpc}]} \\
(4)\end{array}$ & $\begin{array}{c}\text { incl. } \\
{[\mathrm{deg}]} \\
(5)\end{array}$ & $\begin{array}{r}D_{25} \\
{\left[{ }^{\prime}\right]} \\
(6)\end{array}$ \\
\hline ESO300G014 & 47.4077 & -41.0304 & 12.9 & 61.2 & 4.47 \\
\hline ESO300G016 & 47.5436 & -40.0029 & 9.3 & 35.6 & 0.78 \\
\hline ESO302G014 & 57.9204 & -38.4522 & 11.7 & 27.6 & 1.35 \\
\hline ESO357-G007 & 47.6014 & -33.1561 & 17.8 & 72.0 & 1.29 \\
\hline KK98-195 & 200.2841 & -31.5291 & 5.2 & 55.7 & 0.45 \\
\hline KKS2000-23 & 166.55 & -14.4071 & 12.7 & 90.0 & 0.6 \\
\hline NGC1371 & 53.7555 & -24.9332 & 20.4 & 47.5 & 4.9 \\
\hline NGC1592 & 67.4172 & -27.4085 & 13.0 & 64.4 & 1.02 \\
\hline NGC1744 & 74.9908 & -26.0222 & 10.0 & 69.9 & 5.25 \\
\hline NGC3511 & 165.8490 & -23.0867 & 14.2 & 72.6 & 6.03 \\
\hline NGC5068 & 199.7283 & -21.0391 & 6.9 & 30.1 & 7.41 \\
\hline NGC5170 & 202.4532 & -17.9664 & 28.0 & 90.0 & 7.94 \\
\hline NGC5253 & 204.9831 & -31.6401 & 3.0 & 70.1 & 5.01 \\
\hline NGC7424 & 344.3265 & -41.0705 & 13.5 & 32.4 & 5.01 \\
\hline UGCA015 & 12.455 & -21.015 & 3.3 & 67.4 & 1.62 \\
\hline UGCA250 & 178.3502 & -28.5531 & 24.4 & 90.0 & 3.63 \\
\hline UGCA307 & 193.4887 & -12.1058 & 8.6 & 62.0 & 1.82 \\
\hline UGCA320 & 195.8197 & -17.4230 & 7.7 & 90.0 & 6.76 \\
\hline
\end{tabular}

Table 3.1: Positions listed in Columns 2-3 and distances in 4 gathered from NED. Inclination angles from face-on in columns 5 and the optical diameter, $D_{25}$, measured at the $25^{\text {th }}$ mag $\operatorname{arcsec}^{-2}$ isophote in the B-band values taken from HyperLEDA: http://leda.univ-lyon1.fr.

\subsubsection{GBT beam model}

One of the main science goals of this survey is to detect low column density H I around our sample of galaxies. In order to distinguish between low column density $\mathrm{H}$ I from an extragalactic source and the low level radiation entering the sidelobes of 
Table 3.2: H I Measurements and Derived Properties of MHONGOOSE Galaxies

\begin{tabular}{|c|c|c|c|c|c|c|c|c|c|c|c|}
\hline Source & $\begin{array}{c}\sigma_{r m s} \\
{[\mathrm{Jy}]} \\
(2)\end{array}$ & $\begin{array}{c}S_{\mathrm{H} \mathrm{I}} \\
{\left[\mathrm{Jy} \mathrm{km} \mathrm{s}^{-1}\right]} \\
(3)\end{array}$ & $\begin{array}{c}\mathrm{V}_{\mathrm{sys}} \\
{\left[\mathrm{km} \mathrm{s}^{-1}\right]} \\
(4)\end{array}$ & $\begin{array}{c}\mathrm{W}_{50} \\
{\left[\mathrm{~km} \mathrm{~s}^{-1}\right]} \\
(5)\end{array}$ & $\begin{array}{c}\mathrm{W}_{20} \\
{\left[\mathrm{~km} \mathrm{~s}^{-1}\right]} \\
(6)\end{array}$ & $\begin{array}{c}\log _{10} N_{\text {HI } 1 \sigma} \\
{\left[\mathrm{cm}^{-2}\right]} \\
(7)\end{array}$ & $\begin{array}{c}\log _{10} N_{\text {HI } 5 \sigma} \\
{\left[\mathrm{cm}^{-2}\right]} \\
(8)\end{array}$ & $\begin{array}{c}\log _{10} \mathrm{M}_{1 \sigma} \\
{\left[M_{\odot}\right]} \\
(9)\end{array}$ & $\begin{array}{c}\log _{10} \mathrm{M}_{\mathrm{H} \mathrm{I}} \\
{\left[M_{\odot}\right]} \\
(10)\end{array}$ & $\begin{array}{c}\log _{10} \mathrm{M}_{\mathrm{dyn}} \\
{\left[M_{\odot}\right]} \\
(11)\end{array}$ & (12) \\
\hline E300G014 & 0.0104 & 33.6 & 955.0 & 129.2 & 145.2 & 17.35 & 18.33 & 6.69 & 9.39 & 10.36 & 0.108 \\
\hline E302G014 & 0.0096 & 13.7 & 869.3 & 65.5 & 87.1 & 17.31 & 18.29 & 6.56 & 8.92 & 9.91 & 0.102 \\
\hline ESO357G007 & 0.0049 & 15.0 & 1118.3 & 118.4 & 148.5 & 17.02 & 18.0 & 6.64 & 9.32 & 9.91 & 0.256 \\
\hline KK98-195 & 0.0059 & 8.1 & 570.6 & 27.1 & 42.2 & 17.1 & 18.08 & 5.65 & 7.98 & 7.95 & 1.073 \\
\hline N1371 & 0.0076 & 90.9 & 1454.3 & 386.4 & 403.8 & 17.21 & 18.19 & 6.94 & 10.22 & 11.63 & 0.039 \\
\hline N1592 & 0.0048 & 5.8 & 942.9 & 55.1 & 96.2 & 17.01 & 17.99 & 6.35 & 8.63 & 9.34 & 0.194 \\
\hline N1744 & 0.0032 & 174.7 & 743.4 & 191.1 & 207.2 & 16.75 & 17.82 & 5.86 & 9.88 & 10.56 & 0.209 \\
\hline N3511 & 0.0083 & 74.6 & 1130.8 & 272.4 & 308.7 & 17.25 & 18.23 & 6.67 & 9.82 & 11.11 & 0.051 \\
\hline N5068 & 0.0048 & 191.5 & 667.7 & 67.9 & 108.0 & 17.01 & 17.99 & 5.8 & 9.6 & 10.53 & 0.118 \\
\hline N5170 & 0.0062 & 106.2 & 1546.7 & 504.0 & 523.0 & 17.13 & 18.1 & 7.14 & 10.56 & 11.94 & 0.042 \\
\hline UGCA307 & 0.005 & 32.0 & 822.9 & 68.5 & 95.2 & 17.03 & 18.01 & 6.01 & 9.02 & 9.42 & 0.393 \\
\hline UGCA320 & 0.0078 & 133.9 & 749.1 & 106.3 & 126.9 & 17.23 & 18.2 & 6.11 & 9.54 & 10.08 & 0.29 \\
\hline
\end{tabular}

Table 3.2: (1) Source name. (2) measured rms noise. (3) Total integrated flux. (4) Systemic velocity. (5) Linewidth at 50\% maximum. (6) Linewidth at $20 \%$ maximum. (7) $1 \sigma_{r m s}$ column density sensitivity per channel. (8) $3 \sigma_{r m s}$ column density level over a $20 \mathrm{~km} \mathrm{~s}^{-1}$ linewidth. (9) H I mass sensitivity. (10) H I mass. (11) Dynamical mass. (12) Neutral gas fraction. 
the GBT's main beam, we use a beam model which measures these sidelobe levels precisely, modeling the beam response of an unresolved point source. We adopt the beam model used by Pingel et al. (2018). Without this model, emission entering the nearest sidelobes from the main beam could be confused for low column density emission from our sources. For each source in our sample, we used the GBT beam model as a template to regrid each data cube to have $4^{\prime \prime}$ pixels with size $1024 \times 1024$ using the MIRIAD tasks IMGEN and REGRID.

\subsubsection{Integrated intensity images (Moment 0)}

We created integrated intensity images, or Moment 0 images, for each source in our sample. An unmasked image was made by integrating pixel values over a channel range in the data cube containing emission from the central source. We also created masked Moment 0 images with the intention of separating signal from noise when searching for low column density HI. In order to do this, we set to zero the pixels with values below three times the noise in that cube, and again integrate over the same channel range. After integration over the channel range we selected, we expect the $\sigma_{r m s}$ noise in each map (masked and unmasked) to increase by a factor of:

$$
\sigma_{N}=\sqrt{N} \sigma_{r m s}
$$

where $N$ is the number of channels integrated over, and $\sigma_{r m s}$ is the root-mean-squared noise per channel in the data cube. We calculated a $1 \sigma$ noise map for both the masked and unmasked images this way. 
However, because the noise varies by position over the image, we wanted to calculate a more reliable $3 \sigma$ noise threshold. We created a map containing only values above the $3 \sigma$ threshold as sigma varies across the map. We do this in the same way as Pingel et al. (2018) by creating a signal-to-noise (S/N) map for both the masked and unmasked images. The $\mathrm{S} / \mathrm{N}$ map for the unmasked image is made by dividing the unmasked integrated image by the unmasked $1 \sigma$ noise map, while the masked $\mathrm{S} / \mathrm{N}$ map is created by dividing the image where pixels below $3 \sigma_{r m s}$ were set to zero by the masked $1 \sigma$ noise image. From each of these $\mathrm{S} / \mathrm{N}$ maps, we calculated a $3 \sigma$ threshold by taking the mean value of the pixels with $\mathrm{S} / \mathrm{N}$ values falling between 2.75 and 3.25 . We then use this new $3 \sigma$ value to mask, or set to zero, the pixels in our masked integrated image that fall below this value. In doing this, we are confident in our characterization of the noise in our maps.

\subsubsection{Cumulative H I vs. $N_{\mathrm{HI}}$}

We want to find out how much H I mass is contained at different column density levels in each galaxy. In doing this, we will be able to identify the amount of low column density $\mathrm{H}$ I in the galaxy as a percentage of the total H I mass. Accordingly, in the way described in Pingel et al. (2018), we bin the column densities above the $3 \sigma$ level in our unmasked image and calculate the percentage of H I mass that falls into each $N_{\mathrm{HI}}$ bin by converting each pixel in the image to a column density level, and subsequently into an H I mass. We determine the percentage of H I mass at or above a given column density bin, and can therefore quantify the percentage of H I mass 
below some column density threshold. Each galaxy is normalized to that galaxy's maximum H I mass in the cumulative H I vs. $N_{\mathrm{HI}}$ plots in Figures 3.15 through 3.17.

In addition to the data from each cube, we use the beam models described above to determine whether each source can be reliably analyzed. Each beam model for each galaxy is scaled to the maximum column density of the associated GBT image from the data and analyze it in the same way, calculating the cumulative H I mass in each $N_{\mathrm{HI}}$ bin. In doing this, we can determine if the source is following the response of the GBT beam or deviating from it. If the source follows the beam, we can determine that the source is unresolved, and therefore does not satisfactorily fill the beam, causing the H I column density to spread out over the beam area appearing as if low column density H I was detected. To this end, we would omit these unresolved sources from our full statistical analysis. We distinguish which sources are unresolved by looking at the cumulative H I mass ratio of the data versus the model. If the data falls below the beam response of the model, we determine that source to be unresolved. Data above the model is classified as resolved, and if the data is significantly above, we know it is very well resolved.

We can infer additional information about the amount of low column density $\mathrm{HI}$ in the galaxy by looking at the shape of the profile in relation to the GBT beam model. If a source contains an atypical amount of diffuse H I, we will see a deviation in the data profile at low column densities as compared to the beam model. Several of the resolved sources follow the shape of the beam model, and they flatten out at or above the $3 \sigma$ column density threshold described in Section 3.6.3, which is indicative of the scarcity of low column density H I in that galaxy. If, instead, we see an upturn 
at low column densities as compared to the beam model, we are detecting a larger percentage of low column density $\mathrm{H}$ I in that source. This could be the result of a number of scenarios including detection of a low column density companion, extended H I clouds, the presence of a $>3 \sigma$ noise artefact, or the detection of accretion of low column density H I from the CGM. For each image showing a higher percentage of low column density HI, we search for $>3 \sigma$ artefacts, attempting to rule out that possibility, and look for higher $\mathrm{S} / \mathrm{N}$ regions in both the cubes and the integrated images.

\subsubsection{Radial $N_{\mathrm{HI}}$}

We also want to determine how the $N_{\mathrm{HI}}$ behaves as a function of the distance from the galaxy. The optimal way of identifying abundances in low column density $\mathrm{HI}$ is to take the average of the H I column density levels in annuli around the galaxy. This allows us to characterize the azimuthally averaged $N_{\mathrm{HI}}$ at various physical galaxy radii. We define the annuli for each galaxy using the masked rather than unmasked images so as to reduce the effect of quantifying low level noise as signal. Each image is regridded to have $1024 \times 1024$ pixels corresponding to $4^{\prime \prime}$ per pixel for the purpose of precision when quantities inside each annulus are calculated. The GBT beam's FWHM of $9.1^{\prime}$ sets a lower limit to the radius of the smallest annulus to be $4.5^{\prime}$, or $\sim 68$ pixels. The maximum radius of the largest annulus is calculated as the largest multiple of the 68 pixel radius that lies within the image size. For most of our sources, we calculate this to be eight annuli, and for images in which we use a smaller region 
around the galaxy, we end up with five annuli. The $N_{\mathrm{HI}}$ within each annulus is then averaged.

Once again, we want to be able to compare the data against the GBT beam model to look for deviation from the response of the beam. Each model is still scaled to the maximum $N_{\mathrm{HI}}$ value in the image, and drops off at larger radii. The galaxies in our sample follow the response of the beam at low radii, and begin to flatten out around the $3 \sigma$ threshold. Some of the galaxies flatten out above this threshold, which could indicate a smooth extent of the galaxy rather than an ionization edge at a particular column density level around that galaxy. The $1 \sigma$ and $5 \sigma$ thresholds are indicated for each galaxy as the dashed horizontal lines in Figures 3.15 through 3.17.

We can also infer details about the environments of these galaxies by looking at the shape of the radial $N_{\mathrm{HI}}$ profile. If the shape flattens outs due to the $1 \sigma$ noise level of our data, we will not be able to make any substantial claims about the low column density H I at large radii. However, several of the galaxies show an increase in average $N_{\mathrm{HI}}$ at large radii. This could be due to detection of a companion, a higher $\sigma$ noise spike, an extended $\mathrm{HI}$ cloud, or the accretion of $\mathrm{HI}$ onto the galaxy from the CGM. Again, each source is investigated in order to narrow down the source of the increase in average $N_{\mathrm{HI}}$.

\subsubsection{Radial Flux}

We can trace the flux in each galaxy out to the same physical radii that we explored in the radial $N_{\mathrm{HI}}$ analysis using the same method to calculate each annuli. 
We use the unmasked images for this analysis as this is the best way to characterize the total flux of the galaxy at various radii, since our unmasked images contain all flux detected from the source.

Since we are measuring the cumulative flux at physical radii, we should see the profile continue to increase linearly as the area inside each annulus increases linearly if the average $N_{\mathrm{HI}}$ described above is flat. If the radial flux profile rises greater than linearly, we know to look for an increase in low column density gas around that galaxy. A dip in the profile is likely indicative of a negative feature in the image, causing the cumulative flux to decrease at that radius.

\subsection{Results}

Most of the galaxies in our sample are moderately to well-resolved in the GBT beam, and give us reliable statistical properties. A resolved source is defined by a positive offset of the data from the GBT beam model in the cumulative H I mass vs. $N_{\mathrm{HI}}$ plots and in the radially averaged $N_{\mathrm{HI}}$ vs. physical radius plots. The plots in Figures 3.15 through 3.17 show, clockwise from top left, the total integrated H I profile vs. velocity, the cumulative H I mass vs. $N_{\mathrm{HI}}$, the cumulative flux vs. physical radius, and the azimuthally averaged $N_{\mathrm{HI}}$ levels vs. physical radius. The analysis of each galaxy can be seen in Figures 3.15 through 3.17. These results from all of our sources will be described below, and they have been grouped by H I mass bins shown in Figure 3.1. 


\subsubsection{Bin $1\left(6<\log _{10}\left(\mathrm{M}_{\mathrm{HI}}\right)<8 M_{\odot}\right)$}

Figure 3.6 displays a very slight positive offset of ESO 300-16 from the GBT model in the cumulative HI mass vs. column density, where the lowest column density levels deviate in the positive direction. At the smallest radii seen in the radially average $N_{\mathrm{HI}}$ plot, we can see that this source is not well resolved until we move beyond the disk, where the average column density sits around $3 \sigma$.

We see typical responses in the plots of KK98-195 (Figure 3.7). We see a very slight positive deviation of the cumulative H I mass as compared to the GBT beam model at the lowest $N_{\mathrm{HI}}$ level, demonstrating a small fraction of the H I mass in the galaxy to be at the lowest column densities. The average $N_{\mathrm{HI}}$ flattens around $4 \sigma$ in the radially averaged $N_{\mathrm{HI}}$ plot, and the flux increases in the cumulative flux plot.

UGCA015 is $\sim 40^{\prime}$ in angular distance from the large galaxy, NGC 247, which shows up in our data cube at overlapping velocities. For this reason, we analyzed a smaller region around UGCA015 in order to exclude emission from NGC 247. Even still, the cumulative flux increases at large radii, as seen in Figure 3.8.

\subsubsection{Bin $2\left(8<\log _{10}\left(\mathrm{M}_{\mathrm{HI}}\right)<8.5 M_{\odot}\right)$}

Figure 3.9 displays similar behavior to ESO 300-14, where we see an increase at the lowest column densities in the cumulative H I mass plot, flattening around $3 \sigma$ in the radially averaged $N_{\mathrm{HI}}$ plot, and increasing flux levels in the cumulative flux plot.

NGC 1592 has a peak column density level of just over $N_{\mathrm{HI}}=10^{19} \mathrm{~cm}^{-2}$. At the lowest physical radii of this galaxy, it appears to be unresolved in the GBT beam 
until it reaches $\sim 40 \mathrm{kpc}$.

The properties shown in Figure 3.11 are generally what we would expect, with the exception of the slight increase in average column density levels around $25 \mathrm{kpc}$, which is associated with one bright region in the moment 0 image. This bright region has no associated optical counterpart, and is considered a candidate for accretion.

\subsubsection{Bin $3\left(8.5<\log _{10}\left(\mathrm{M}_{\mathrm{HI}}\right)<9 M_{\odot}\right)$}

ESO 357-007 follows the GBT beam more closely at higher column densities, with increasing amounts of diffuse $\mathrm{H}$ I beginning around $N_{\mathrm{HI}}=10^{18.8} \mathrm{~cm}^{-2}$. We can see in the azimuthally averaged $N_{\mathrm{HI}}$ plot that the galaxy does not appear to be resolved in the GBT beam at low physical radii of the galaxy, and flattens around the $5 \sigma$ level. The cumulative flux does show a non-linear increase at larger physical radii. In the absence of evidence of a companion galaxy, or any bright artefact in the data cube causing the cumulative flux to increase, we are led to believe that this could be evidence for neutral hydrogen at large radii accreting from the CGM onto ESO 357007.

KKS2000-23 appears to be moderately resolved in the GBT beam, and shows a significant portion of its H I mass to be made up of low column density gas, as can be seen in the cumulative H I mass plot in Figure 3.13. However, inspection of the data cube and the moment map reveal artefacts, making it difficult to determine if the low column density gas comes from real emission or artefacts of the reduction. This particular issue could be alleviated with additional observations to verify the 
presence or absence of low $N_{\mathrm{HI}}$ gas.

UGCA307 also shows an increase in low column density gas in Figure 3.14. However, the azimuthally averaged $N_{\mathrm{HI}}$ vs. physical radius displays a steep increase between $55 \mathrm{kpc}$ and $65 \mathrm{kpc}$. We inspected the data cube and discovered a small companion galaxy, LCRS B125208.8-112329 residing $72 \mathrm{kpc}$ away in projected distance. This can also be seen in the cumulative flux vs. physical radius plot, where the flux increases around the same physical distance. This too, would be the first redshift information available for this galaxy.

\subsubsection{Bin $4\left(9<\log _{10}\left(\mathrm{M}_{\mathrm{HI}}\right)<9.5 M_{\odot}\right)$}

ESO 300-14 is an example of a typical source in this sample. The total integrated flux of ESO 300-14 is shown in Figure 3.15, where we see the expected double horn feature for an inclined, rotating galaxy. It is resolved in the GBT beam, where the cumulative flux follows the shape of the GBT beam model of a point source, but is positively offset in relation to that model. The azimuthally averaged column densities vs. physical radius show that at the lowest radii, this source follows the model, but flattens out around the $3 \sigma-5 \sigma$ threshold, and the cumulative flux at various physical radii increases as expected.

Similarly, the face-on galaxy, NGC 5068, exhibits typical behavior when comparing these same properties. The cumulative H I mass plot in Figure 3.16 shows that $\sim 99 \%$ of the mass in the galaxy is seen in column densities above $N_{\mathrm{HI}}=10^{19} \mathrm{~cm}^{-2}$. This is substantiated in both the average $N_{\mathrm{HI}}$ and cumulative flux vs. physical radii 
plots, where there is no evidence of an increase in $\mathrm{H}$ I at large radii.

We detected two companions in the region close to UGCA320: UGCA319 at $\sim 41 \mathrm{kpc}$, and a second detection around $\sim 61 \mathrm{kpc}$ projected distances. The closer companion, UGCA319 can be seen as a slight bump in the averaged $N_{\mathrm{HI}}$ around $\sim 45$ kpc in Figure 3.17. The second H I detection lacks a known counterpart, but may represent a detection of LEDA 886203, the only potential galaxy at this position. These two companions are likely what is causing the gradual increase in cumulative H I flux at radii larger than $40 \mathrm{kpc}$. This would also be the first redshift information available on LEDA 886203.

\subsubsection{Bin $5\left(9.5<\log _{10}\left(\mathrm{M}_{\mathrm{HI}}\right)<10 M_{\odot}\right)$}

NGC 1371 is well-resolved in the beam, as seen in the cumulative H I mass plot in Figure 3.18. In most of these plots, NGC 1371 behaves as expected, with the exception of an artefact at $\sim 150 \mathrm{kpc}$ as seen in the radially averaged $N_{\mathrm{HI}}$ plot.

One of our more massive galaxies, NGC 1744, shows no signs of excess amounts of low column density gas as seen in the cumulative H I mass plot in Figure 3.19. We can see in the radially averaged $N_{\mathrm{HI}}$ plot that this source is well-resolved, and shows an unusually high average $N_{\mathrm{HI}}$ at radii beyond the disk. This could be indicative of accreting H I or a higher noise floor than we had estimated.

NGC 3511 shows large deviation at low column density levels, where the cumulative amount of HI increases almost linearly through $N_{\mathrm{HI}}=10^{18.6} \mathrm{~cm}^{-2}$. It appears to be resolve in the GBT beam in both the cumulative mass plot and the averaged 
$N_{\mathrm{HI}}$ plot.

NGC 5170, in contrast with NGC 1744 which resides in the same mass bin, does show an increase towards lower column density H I mass. Figure 3.21 reveals a sharp change in the slope of the cumulative HI mass at column densities lower than $\sim 10^{19.4} \mathrm{~cm}^{-2}$. The azimuthally averaged $N_{\mathrm{HI}}$ plot does show an increase in average $N_{\mathrm{HI}}$ around $175 \mathrm{kpc}$. Inspection of the moment 0 image reveals some bright regions at the same physical distance, which could be accreting H I gas. Without any indication of nearby companions associated with those bright regions which appear to lack optical counterparts, we consider this a candidate for accretion from the CGM.

3.7.6 Bin $6\left(10<\log _{10}\left(\mathrm{M}_{\mathrm{HI}}\right)<11 M_{\odot}\right)$

NGC 7424, one of the most H I massive galaxies in our sample, displays expected behavior in all plots in Figure 3.22, with the exception of slightly higher average $N_{\mathrm{HI}}$ values seen in the averaged $N_{\mathrm{HI}}$ vs. physical radius plot. This is likely due to a bright companion detected in the data cube.

One of our largest sources, UGCA250, occupies our highest mass bin: $10<$ $\log _{10}\left(\mathrm{M}_{\mathrm{HI}}\right)<11 M_{\odot}$. The cumulative H I mass increases at low column densities, and the cumulative flux continues to grow as the radius increases. Inspection of the data cube revealed a bright, positive stripe across the top of UGCA250, at the same declination as another bright source on the edge of our cube, UGCA247. Within that stripe is a possible new $\mathrm{HI}$ detection which is coincident with the only catalogued galaxy within the size of the GBT beam at this position, FLASH J115508.00-282045.1 
at a radius of $\sim 185 \mathrm{kpc}$.

\subsection{Discussion}

\subsubsection{Extraplanar gas}

Our analysis of the cumulative $\mathrm{HI}$ mass allowed us to quantify the total H I mass in the moment maps. Our maps were made at a constant angular size of $2^{\circ} \times 2^{\circ}$, so we do not have a consistent physical region around each mapped source where map sizes range from $105-977 \mathrm{kpc}$ on one side, however we can subtract off the H I mass of the disk using the mass derived from the spectra of each galaxy. The total H I mass less the disk mass gives us some value for the amount of mass outside the disk. Out of our 18 galaxies, only six contained an excess amount of gas outside the disk, ranging from $2 \%$ to $45 \%$ more H I. Interestingly, five of these are some of our lowest $M_{\mathrm{HI}}$ galaxies (ESO 300-16, ESO 302-14, KK98-195, KKS2000-23, NGC 1592), and one is our highest (NGC 5170).

\subsubsection{Fraction of low $N_{\mathrm{HI}}$}

We define a characteristic column density as low $N_{\mathrm{HI}}$ if it is below a level of $10^{19} \mathrm{~cm}^{-2}$. This value is taken from the prediction that below $\sim 10^{19} \mathrm{~cm}^{-2}$ the amount of hydrogen in the neutral phase is truncated, and the gas transitions from mostly neutral to mostly ionized, making detections much below this level difficult, although not impossible. This sharp drop is largely due to photoionization of the neutral

hydrogen by the extragalactic radiation field and represents one way of defining the 

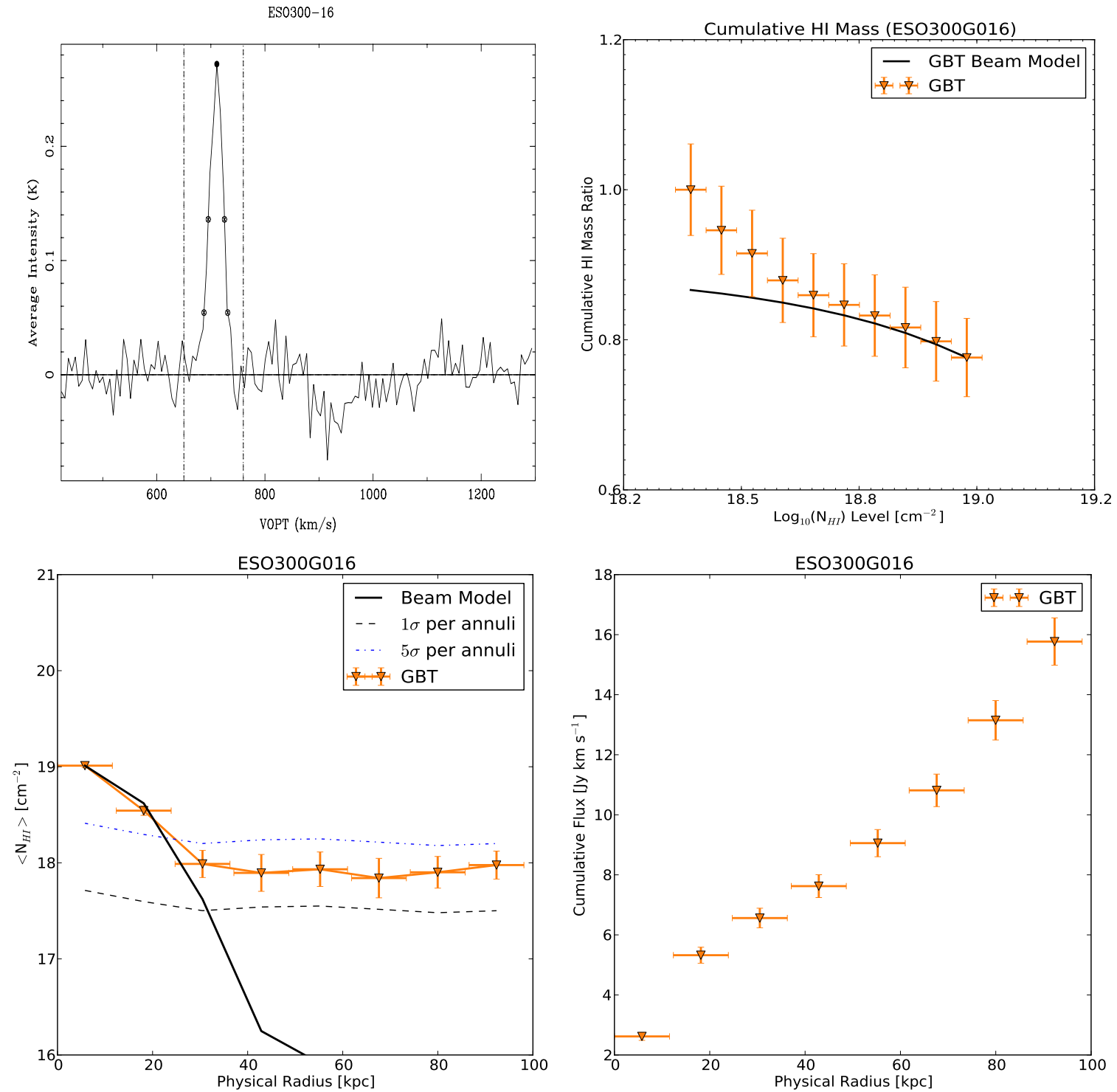

Figure 3.6: Top left: Total integrated H I profile measured between the dashed and dotted vertical lines. Open circles and $\times$ 's indicate $20 \%$ and $50 \%$ maximum velocity widths. Filled circles represent the mean velocity. Top right: Cumulative H I mass. The total H I mass in the moment 0 map is plotted by the fraction of gas in column density bins, and compared to the GBT beam model, scaled to the peak column density. Lower left: Azimuthally averaged $N_{\mathrm{HI}}$. Column density averaged over annuli extending radially from the center of the galaxy and compared to the GBT beam model. The black dashed line characterizes the $1 \sigma$ noise in each annulus, and the blue dot-dash line represents the $5 \sigma$ noise in each annulus. Lower right: Cumulative flux. Flux in each of those annuli are summed to obtain a measure of the total flux out to the edge of each map. 

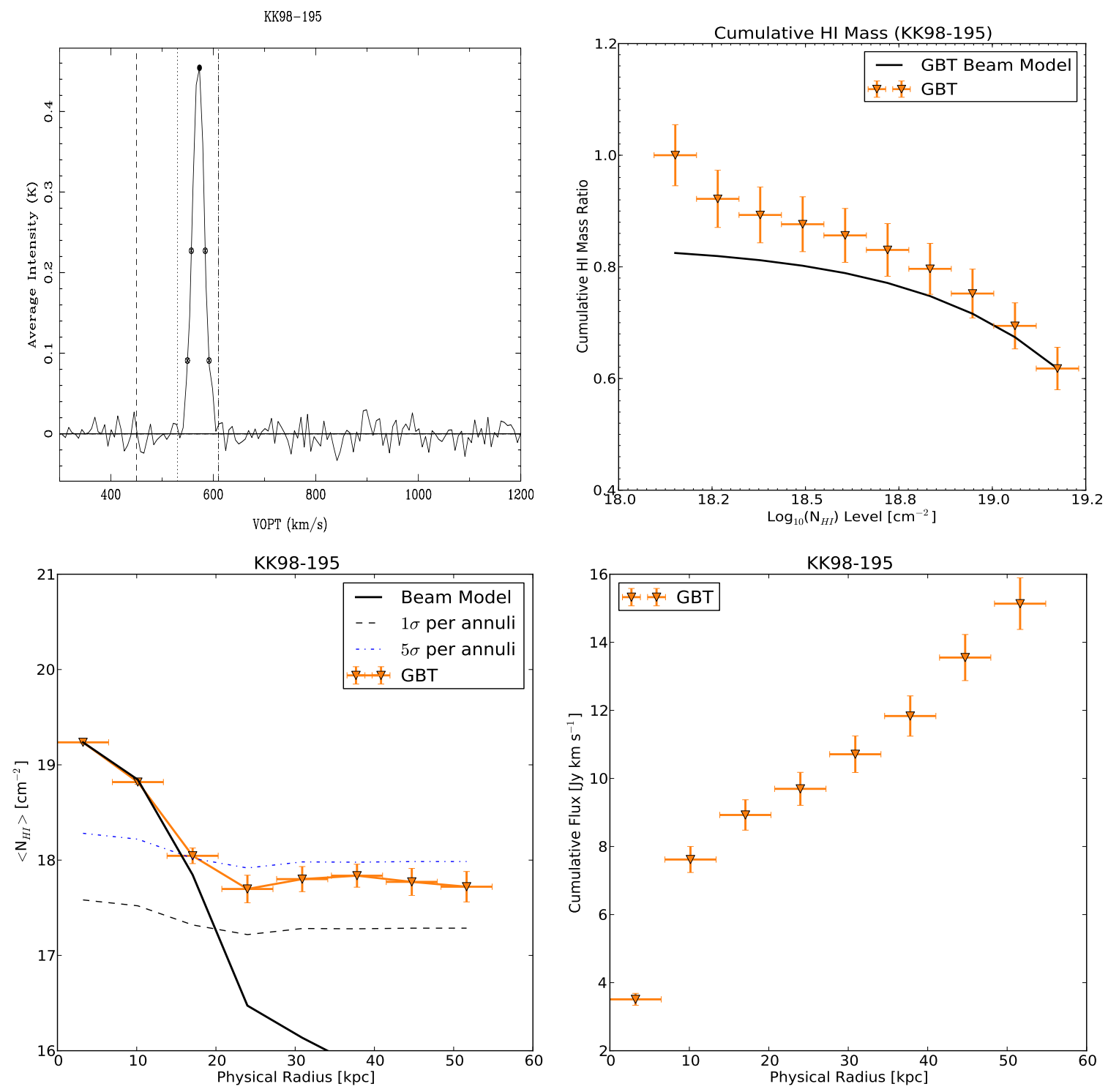

Figure 3.7: Same as Figure 3.6 for KK98-195. 

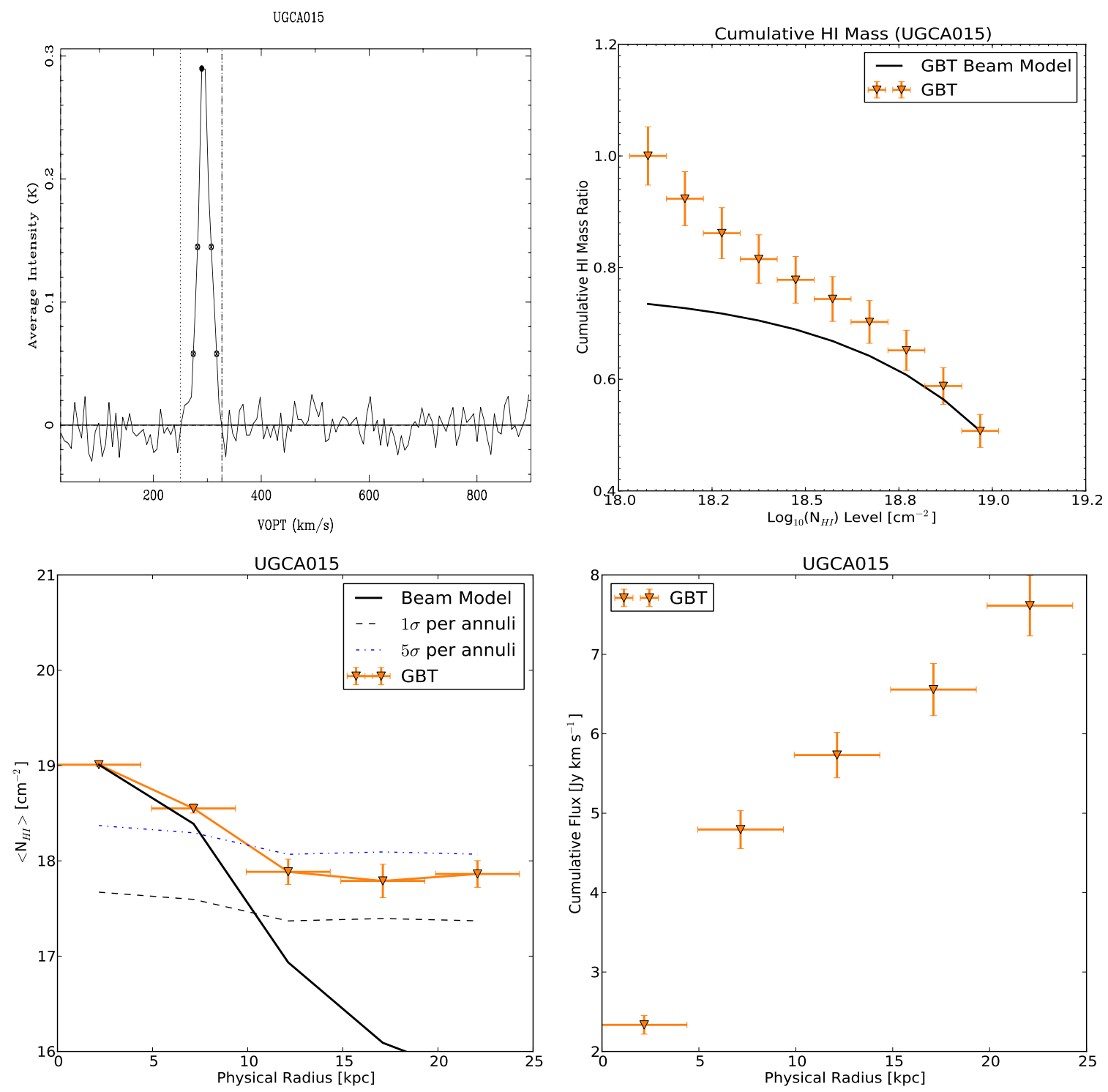

Figure 3.8: Same as Figure 3.6 for UGCA015. 

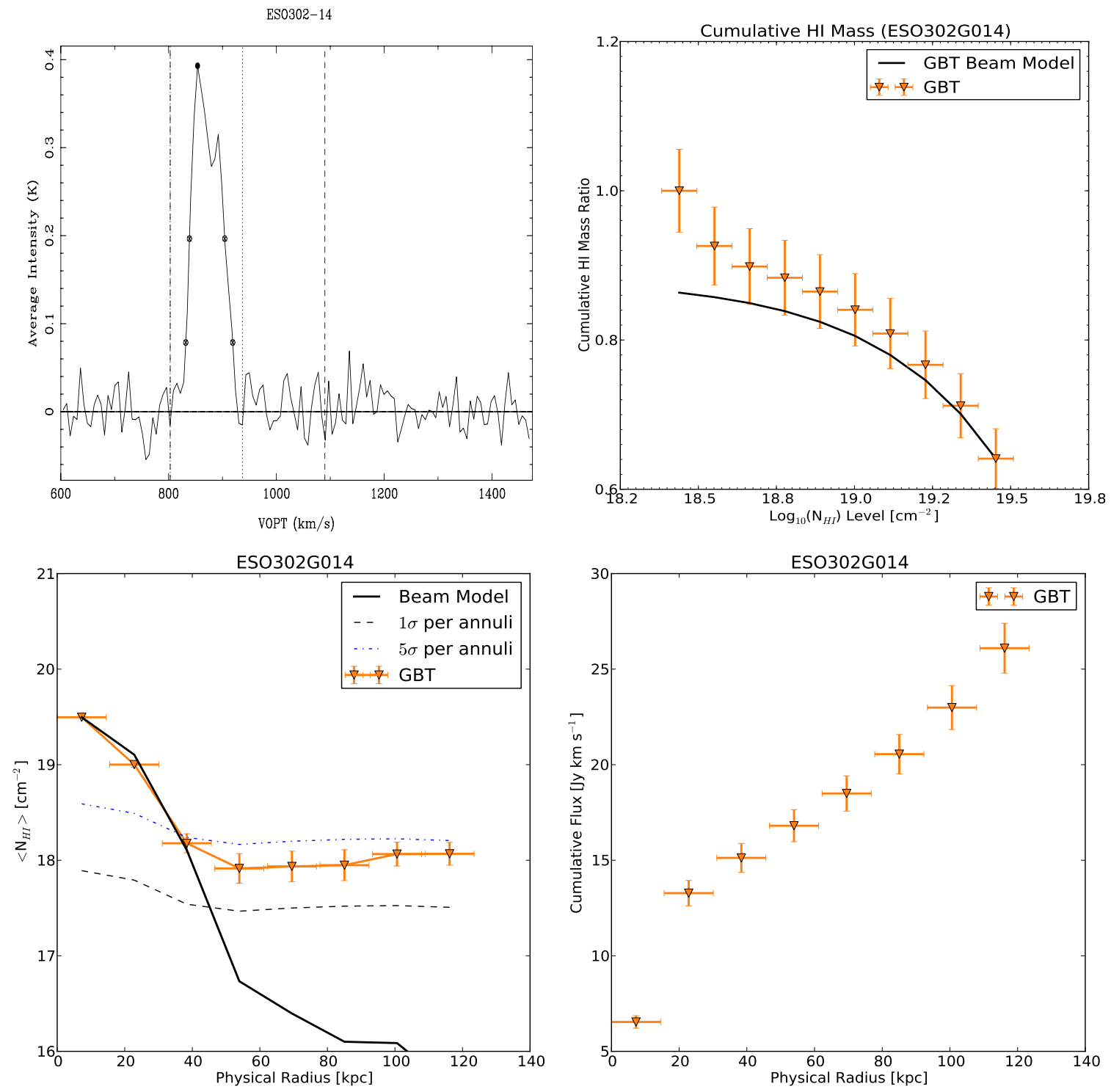

Figure 3.9: Same as Figure 3.6 for ESO 302-14. 

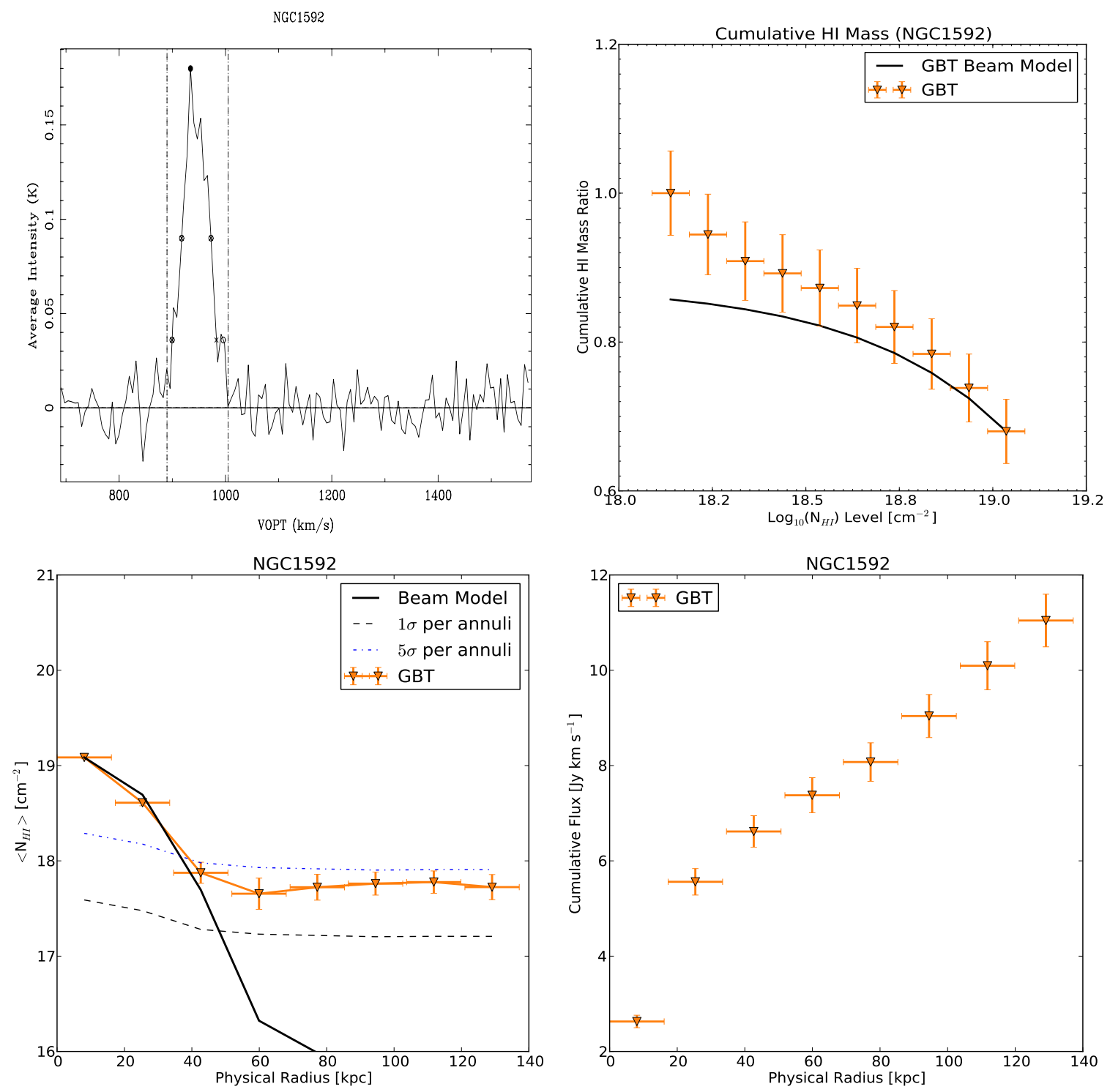

Figure 3.10: Same as Figure 3.6 for NGC 1592. 

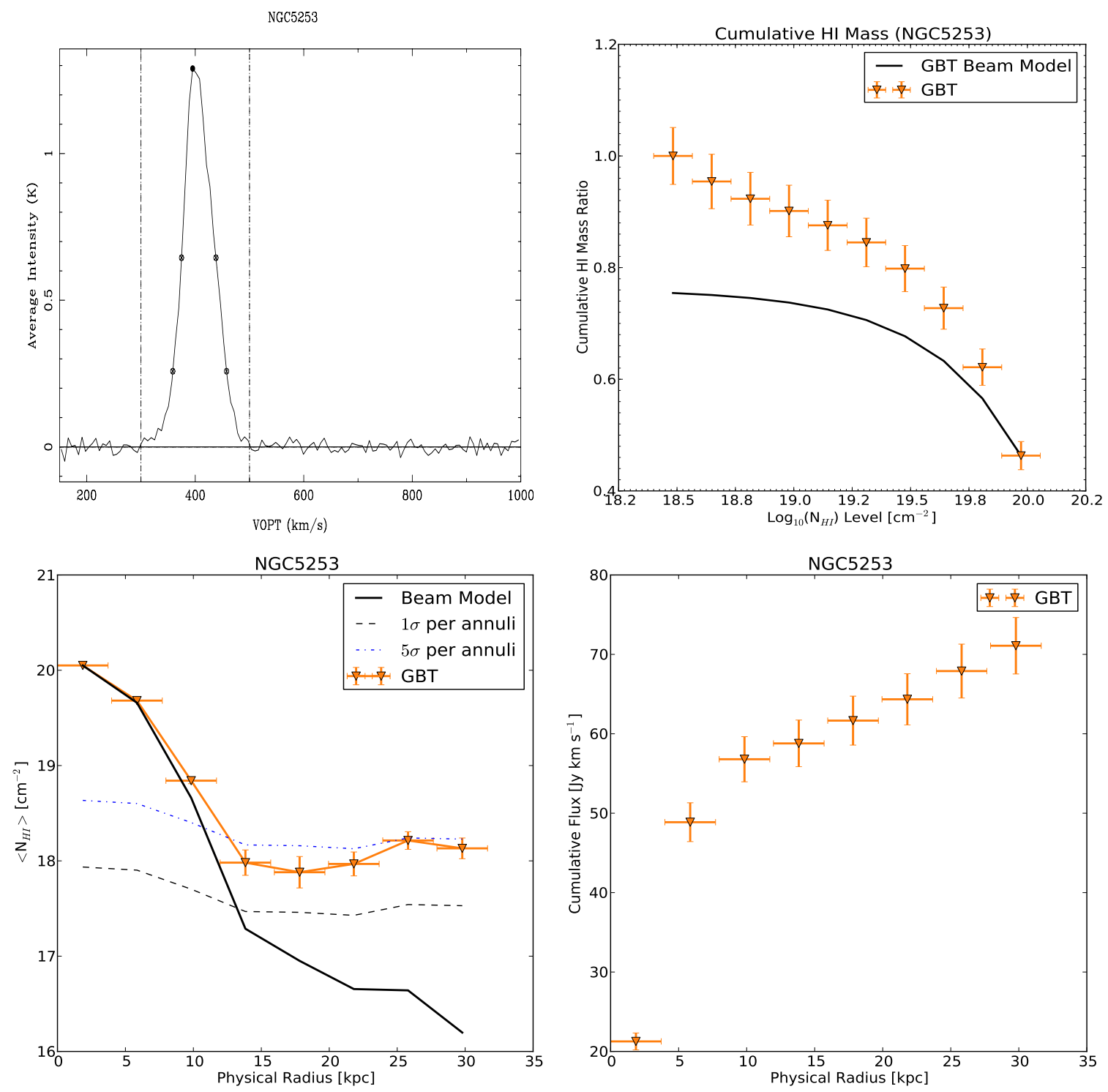

Figure 3.11: Same as Figure 3.6 for NGC 5253. 

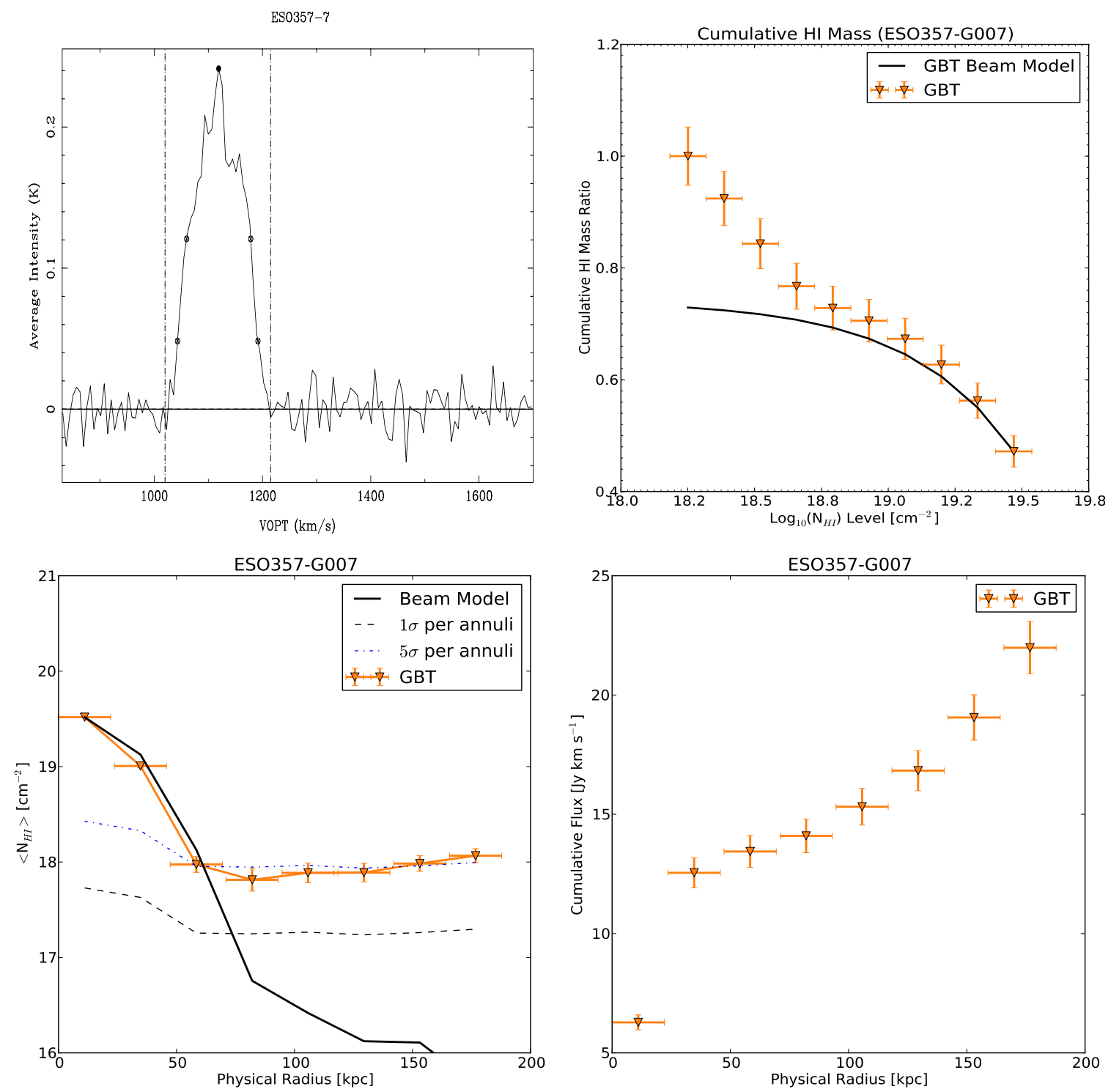

Figure 3.12: Same as Figure 3.6 for ESO 357-007. 

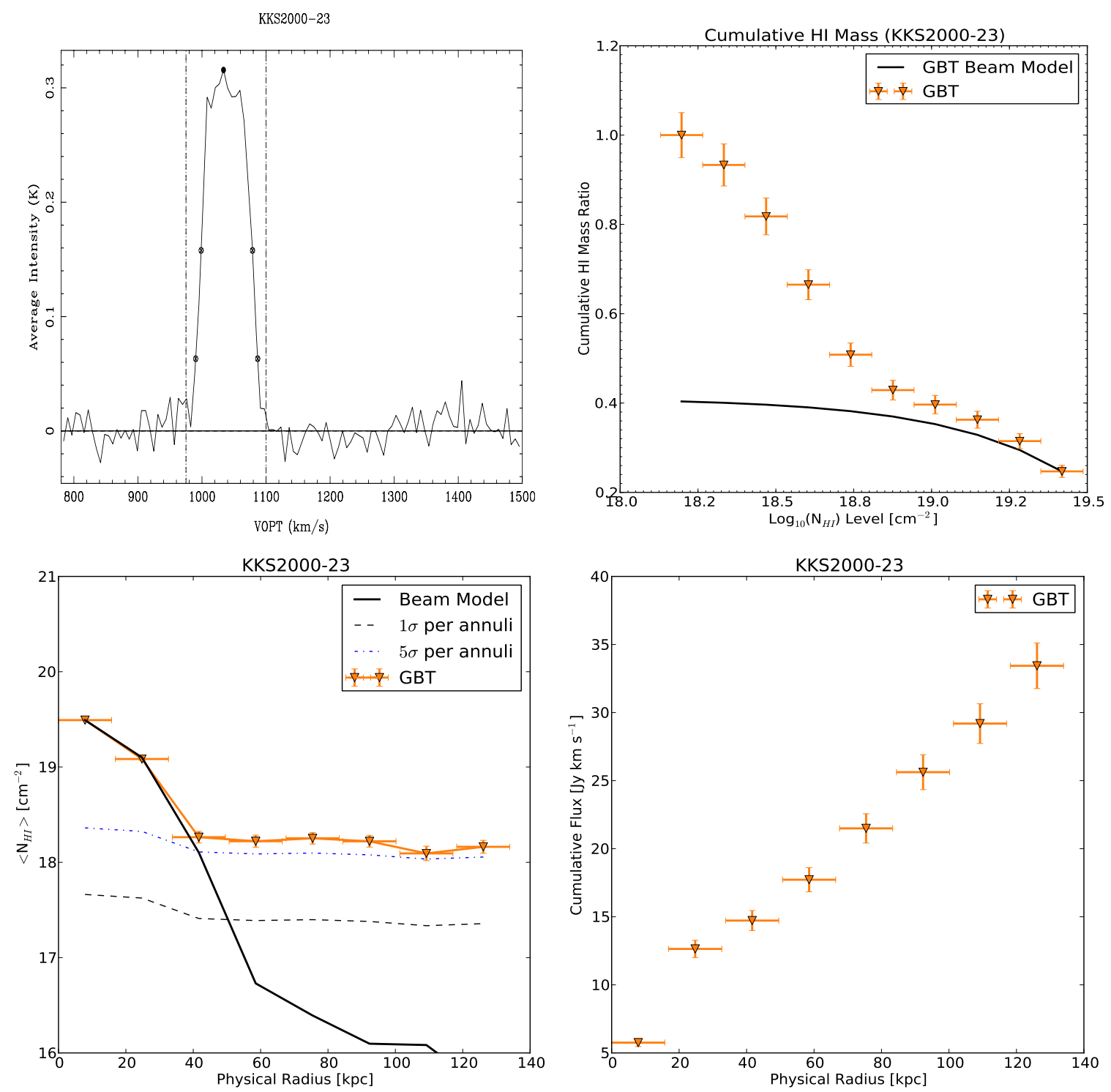

Figure 3.13: Same as Figure 3.6 for KKS2000-23. 

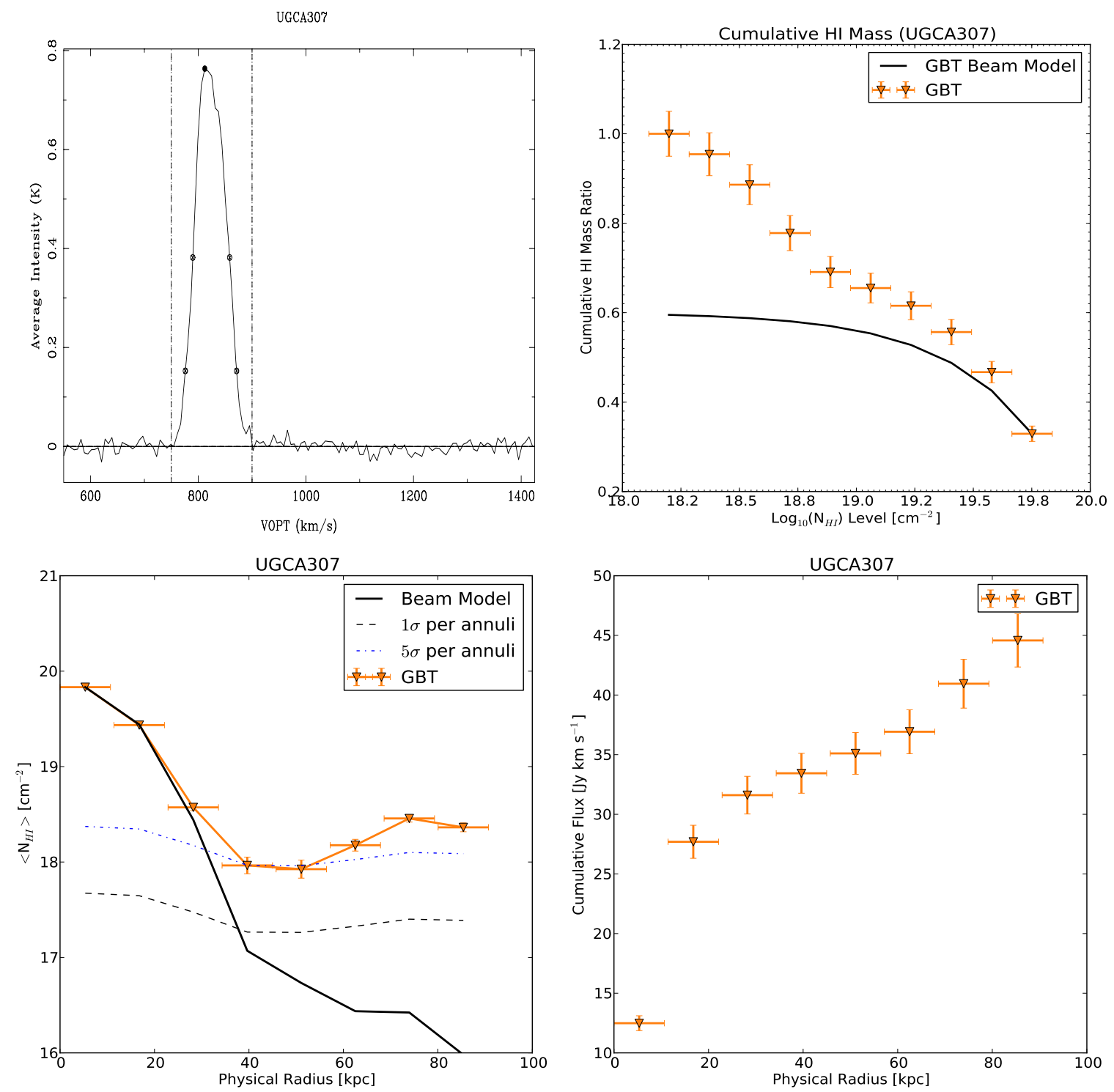

Figure 3.14: Same as Figure 3.6 for UGCA307. 

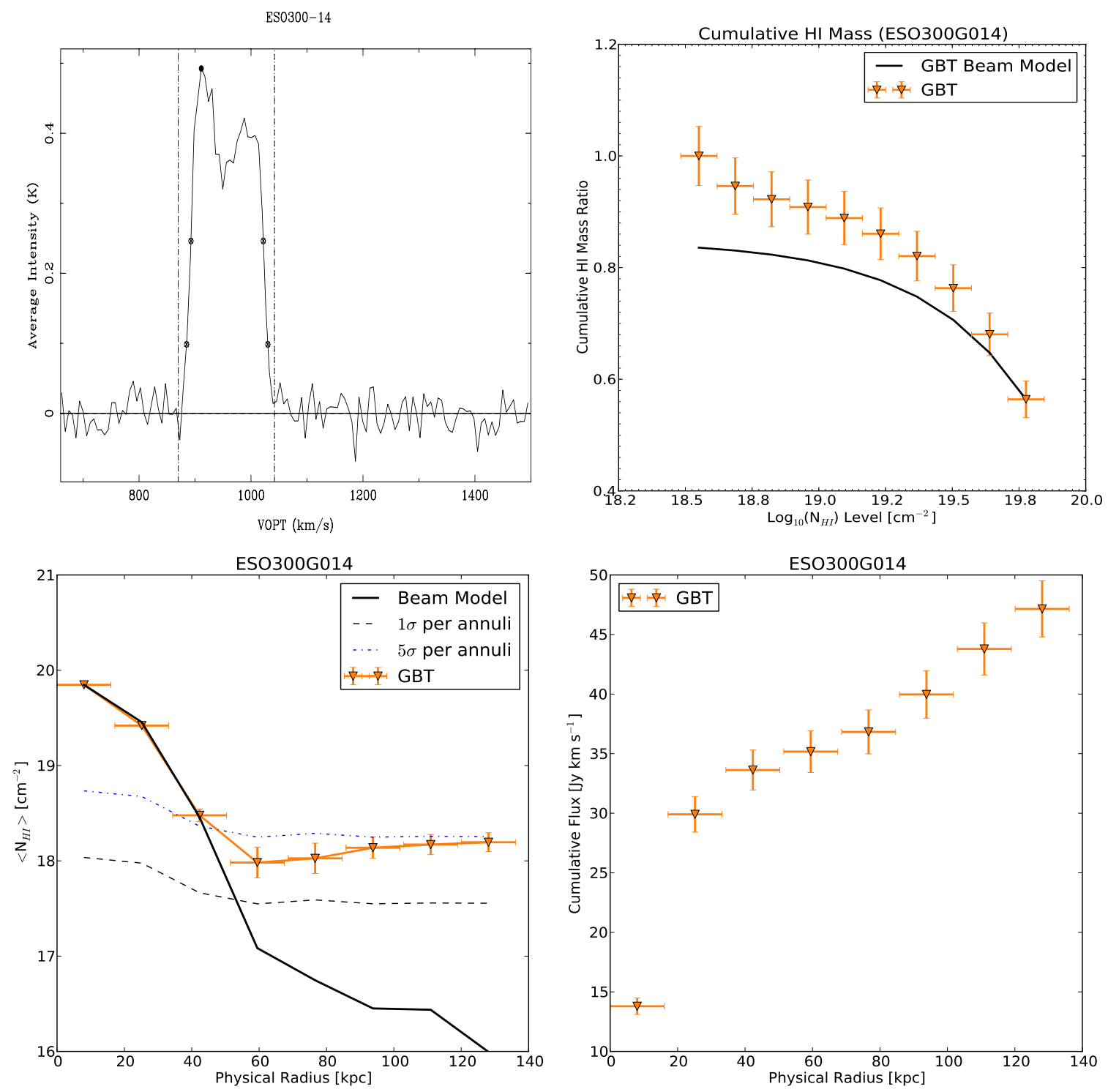

Figure 3.15: Same as Figure 3.6 for ESO300-14. 

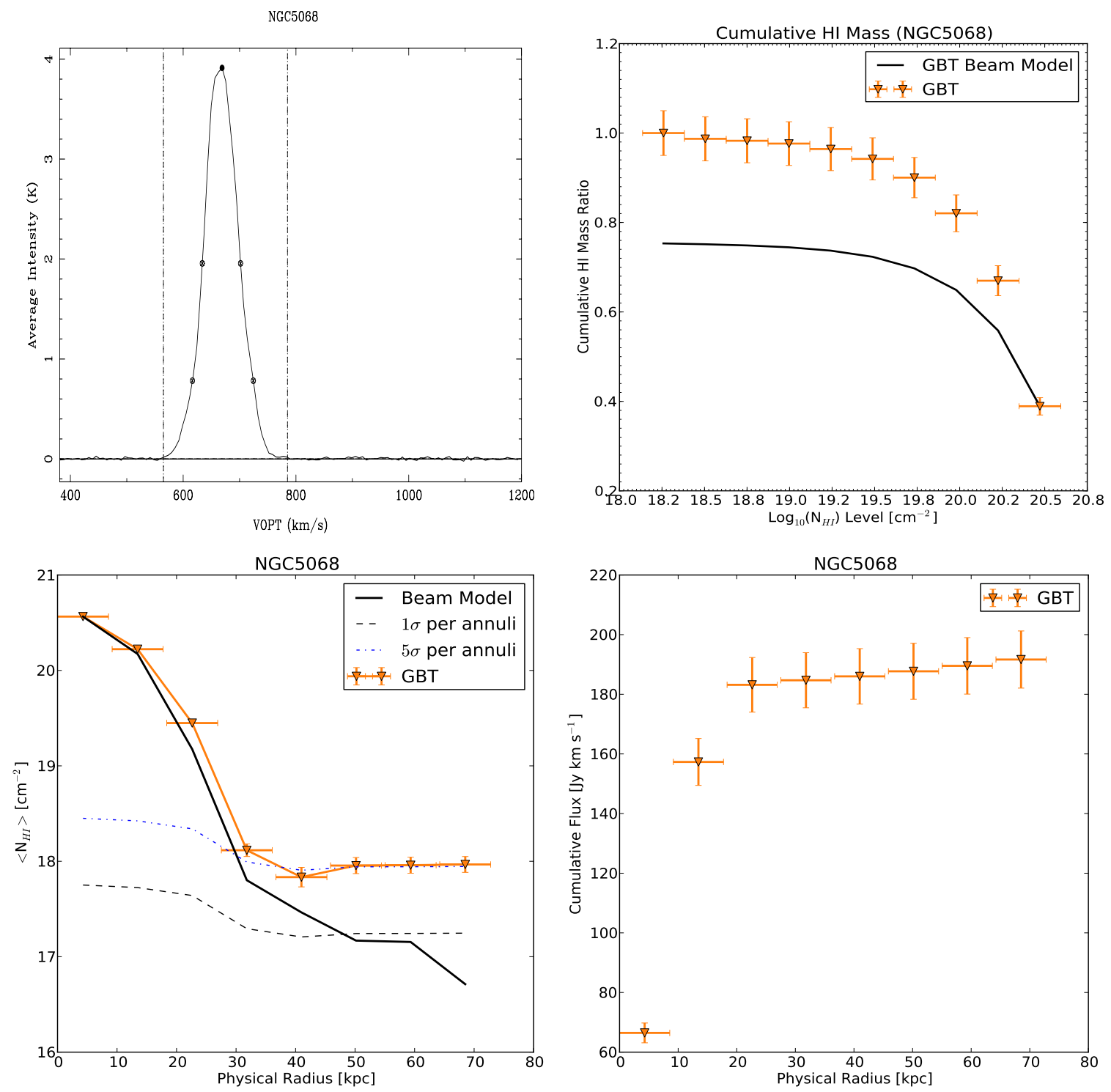

Figure 3.16: Same as Figure 3.6 for NGC 5068. 

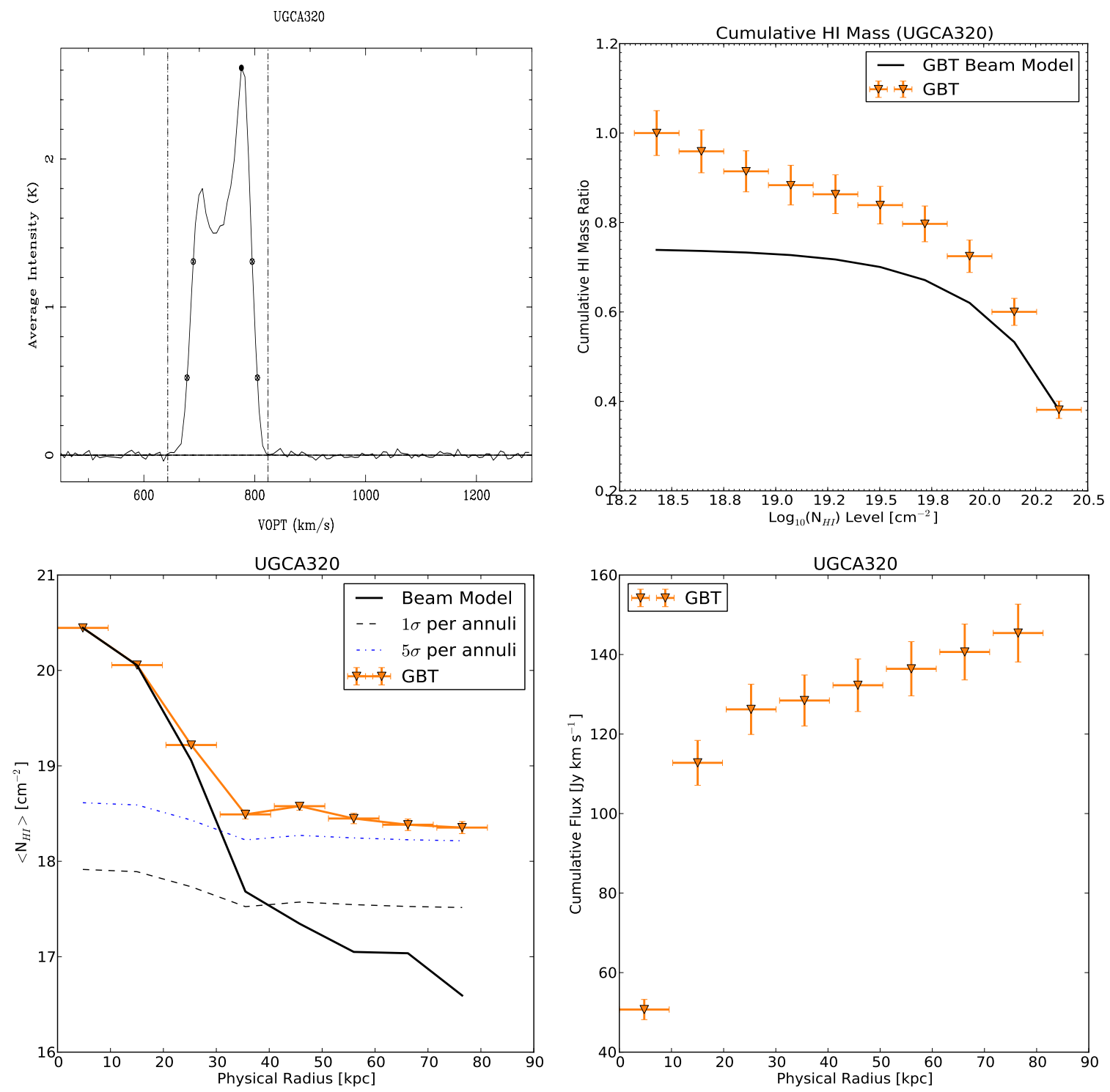

Figure 3.17: Same as Figure 3.6 UGCA320. 

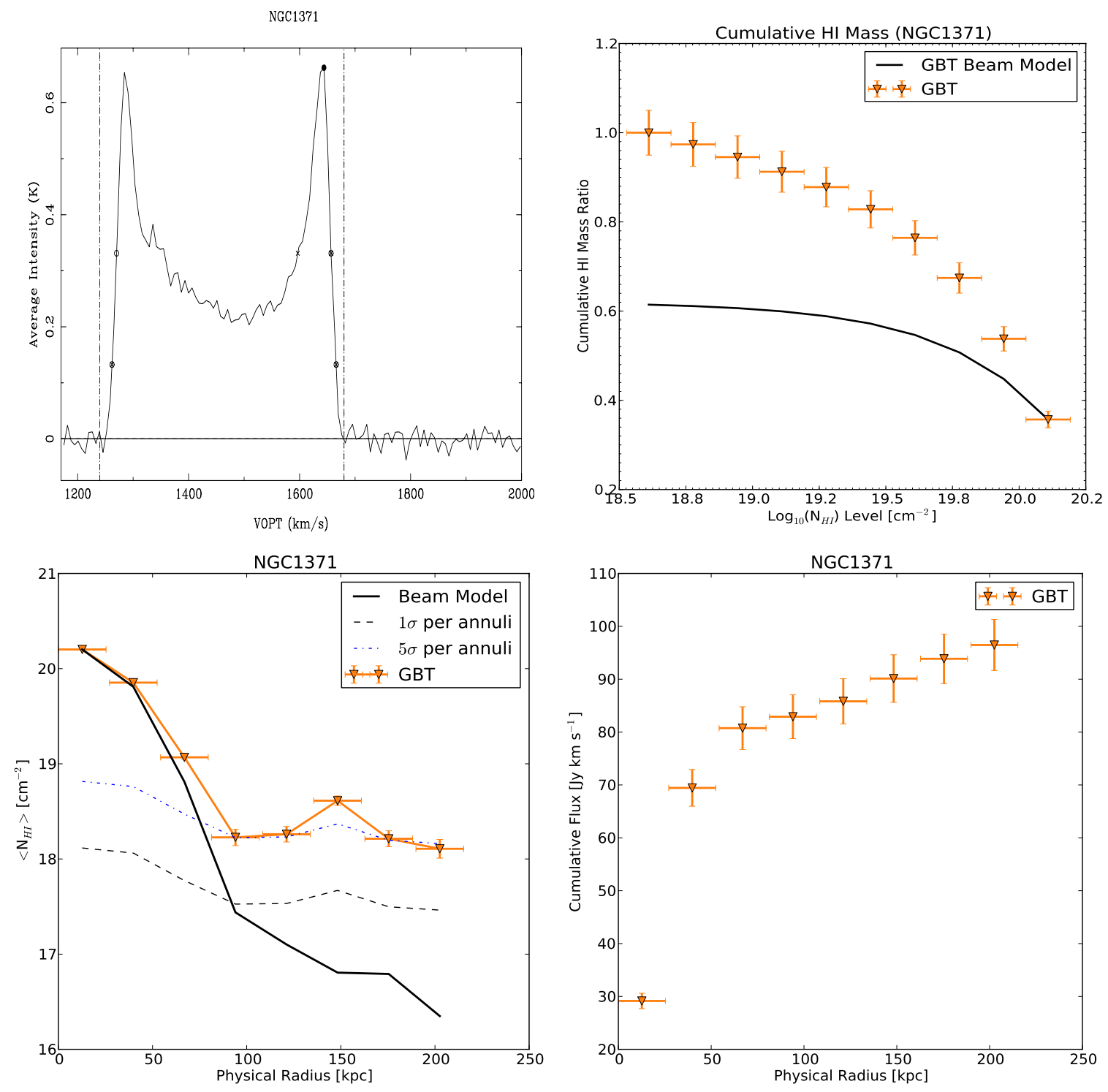

Figure 3.18: Same as Figure 3.6 for NGC 1371. 

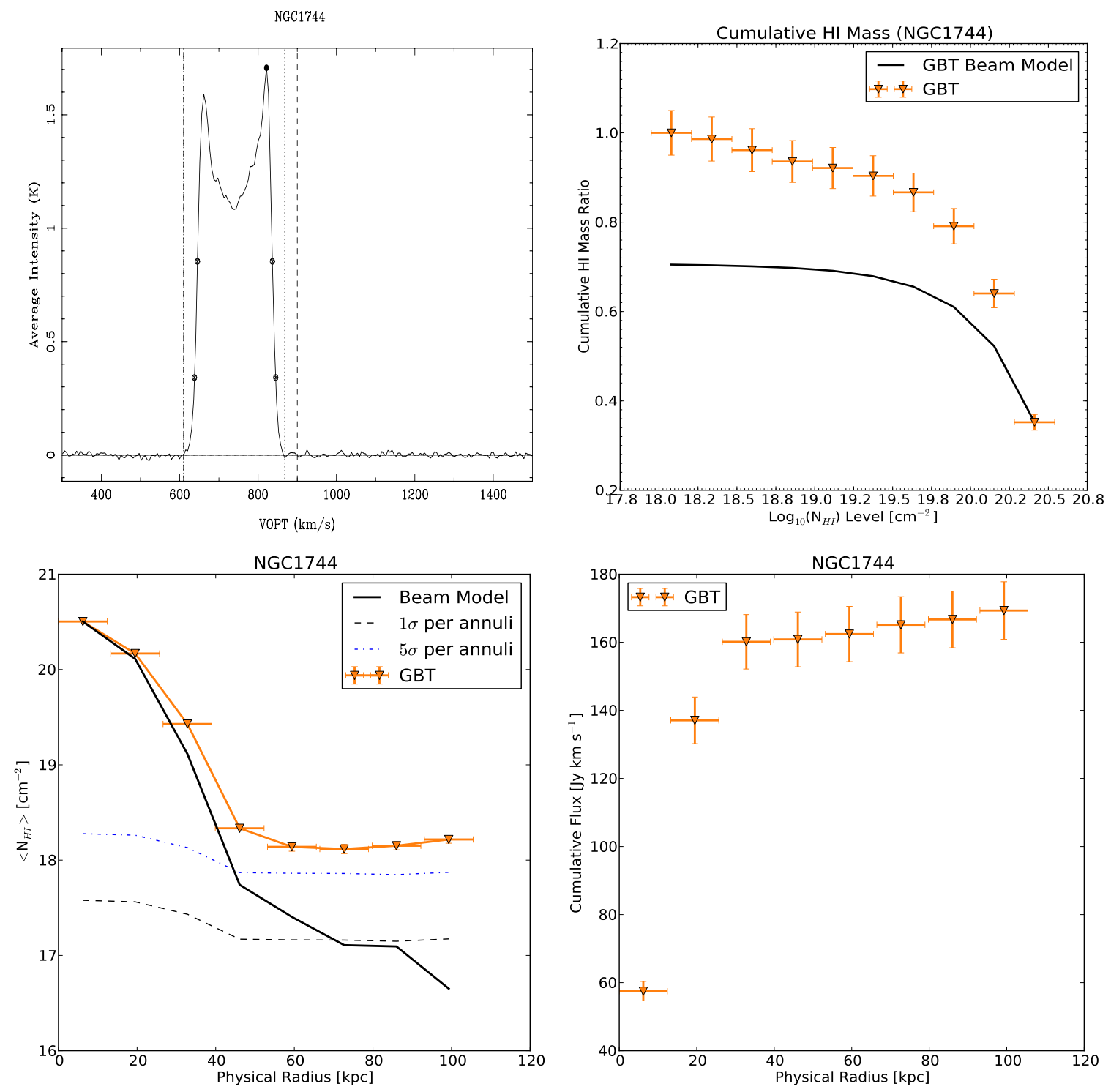

Figure 3.19: Same as Figure 3.6 for NGC 1744. 

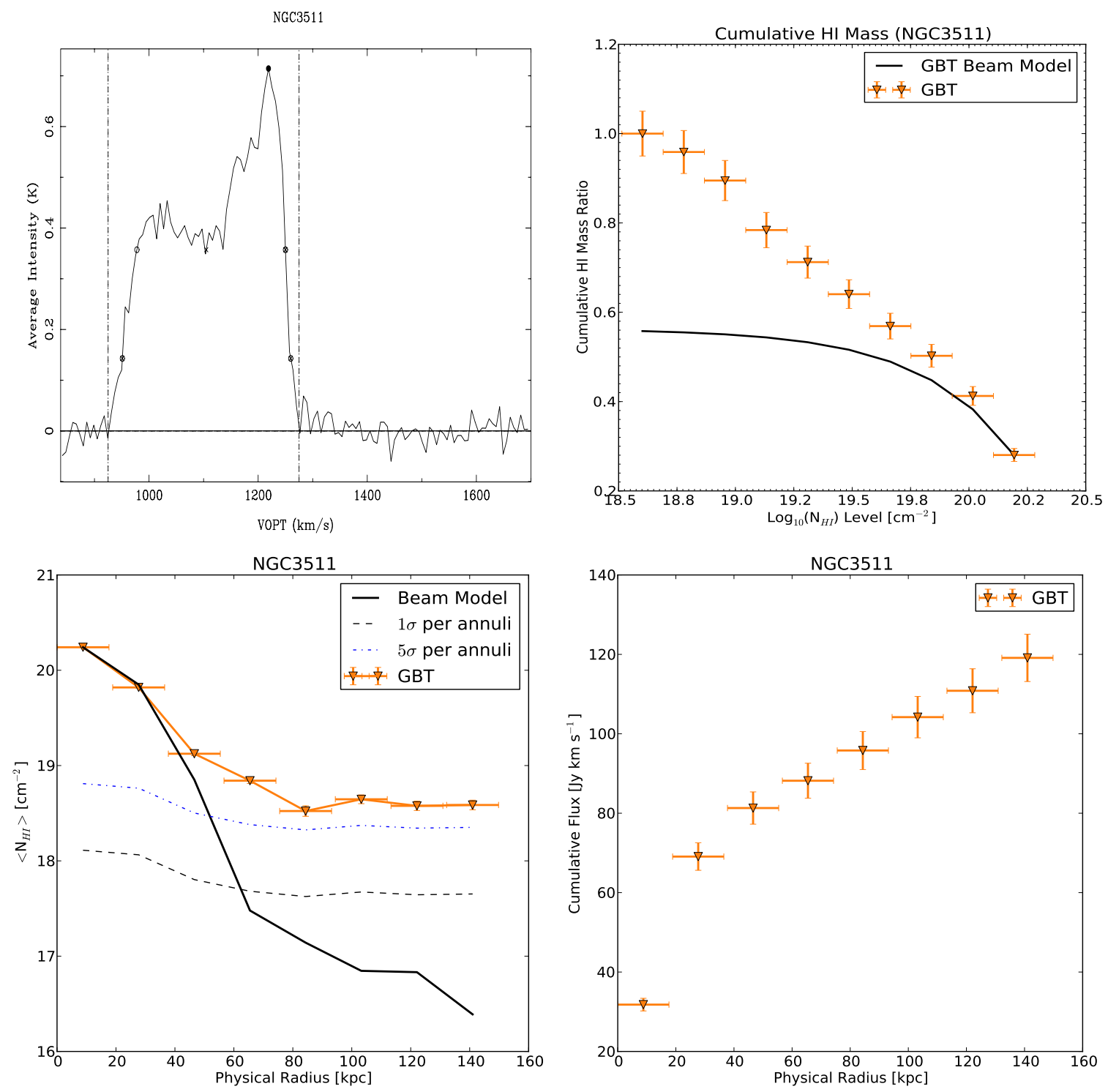

Figure 3.20: Same as Figure 3.6 for NGC 3511. 

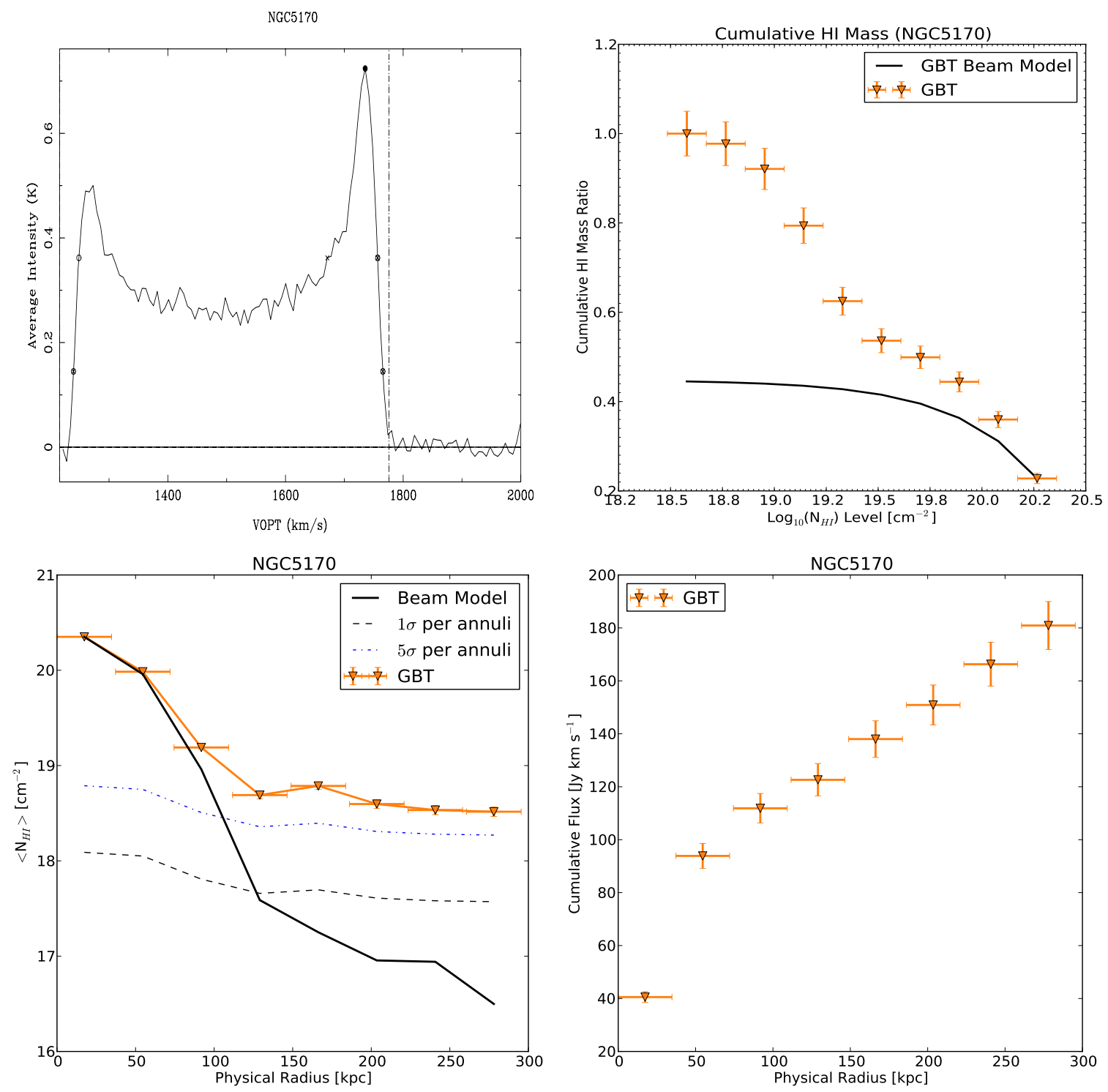

Figure 3.21: Same as Figure 3.6 for NGC 5170. 

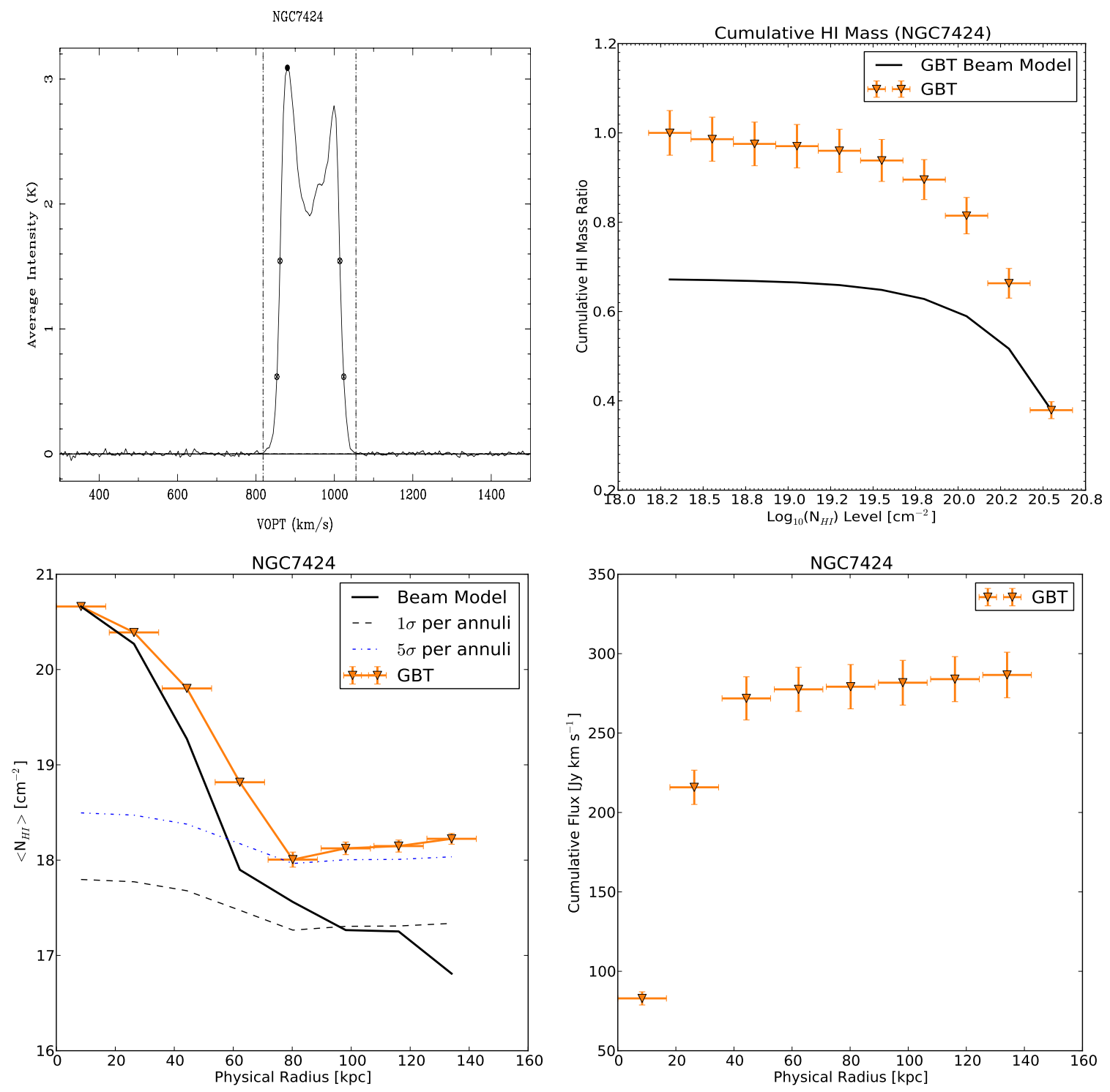

Figure 3.22: Same as Figure 3.15 for NGC 7424. 

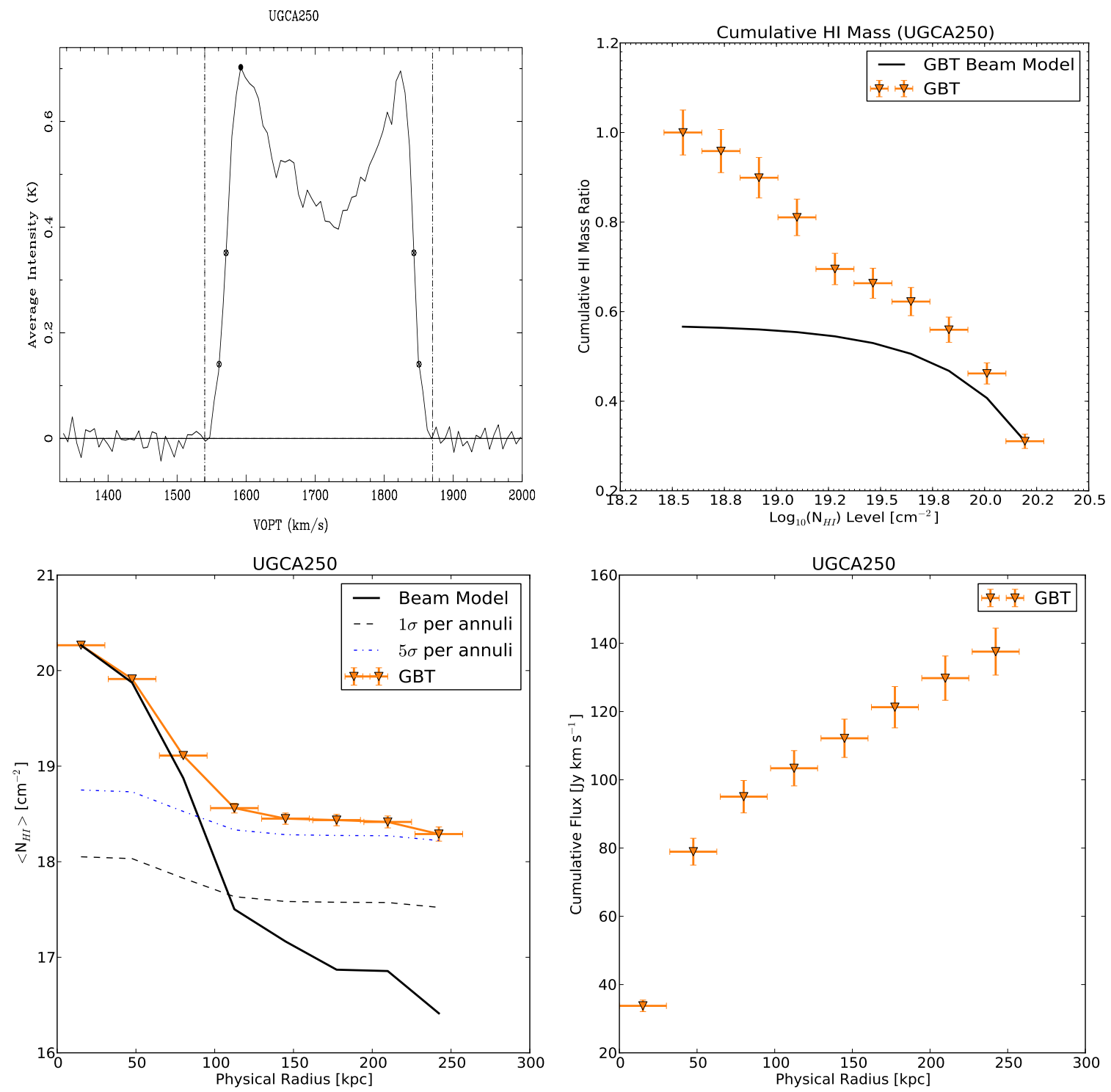

Figure 3.23: Same as Figure 3.6 for UGCA250. 
edge of an H I disk. This was first explained by Bochkarev \& Siuniaev (1977) and later by Maloney (1993) who suggested that the ionization fraction sharply increases at a critical column density of a few times $10^{19} \mathrm{~cm}^{-2}$, irrespective of galaxy mass or halo parameters. An example of this sharp drop-off in H I is the KAT-7 observations of M83, which revealed a steep decrease in $N_{\mathrm{HI}}$ at the edge of the galaxy disk (Heald et al., 2016). Recently, Bland-Hawthorn et al. (2017) demonstrated that it may be possible to detect low column density H I at significantly larger radii than the predicted truncation radius from Maloney (1993). Specifically, they showed that the radius at which $50 \%$ of the total amount of hydrogen is ionized is significantly larger. These results seem to be congruent with those of Ianjamasimanana et al. (2018) who investigated radially averaged column density profiles of 17 galaxies in search of a break at the $\sim 10^{19} \mathrm{~cm}^{-2}$ level and found no evidence for a sharp change anywhere above their detection limits.

We determined the diffuse neutral fraction for each galaxy using (Pingel et al., 2018):

$$
f_{19}=1-\frac{M_{19}}{M_{\mathrm{H}}} .
$$

In this equation, the diffuse neutral fraction, $f_{19}$, is defined by the fraction of $\mathrm{HI}$ below column densities of $10^{19} \mathrm{~cm}^{-2}$, where $M_{19}$ is the H I mass at column density levels $N_{\mathrm{HI}} \geq 10^{19} \mathrm{~cm}^{-2}$ and $M_{\mathrm{HI}}$ is the total H I mass. Values of $f_{19}$ are listed in Table 3.3 .

With our $f_{19}$ values in hand, we would like to compare the diffuse neutral 

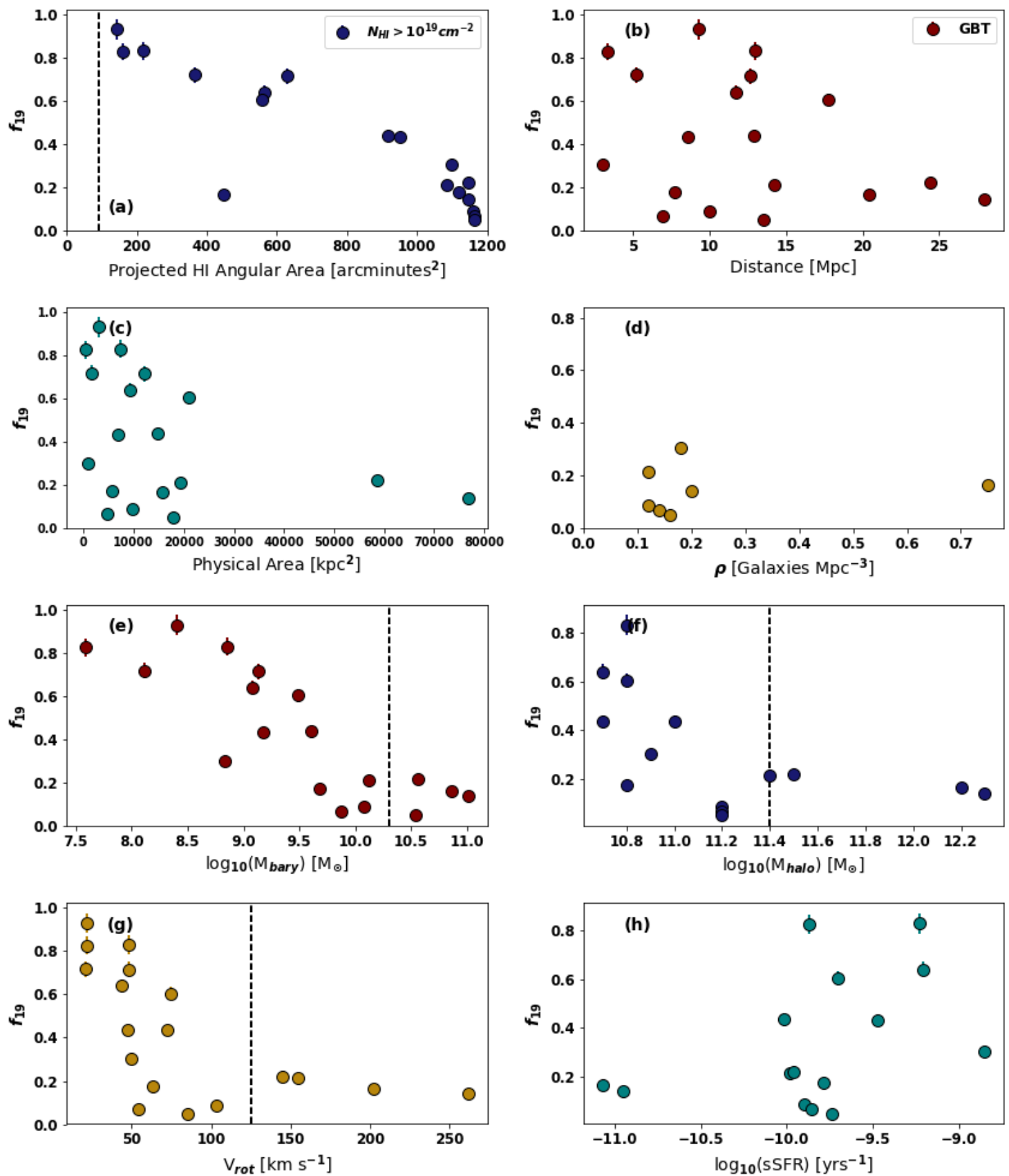

Figure 3.24: Comparisons of the diffuse fraction of $\mathrm{H} \mathrm{I}$ in the region surrounding each of our MHONGOOSE galaxies with: (a) Angular areas calculated inside region where column densities reach $N_{\mathrm{HI}}>5 \times 10^{18} \mathrm{~cm}^{-2}$ and $N_{\mathrm{HI}}>1 \times 10^{19} \mathrm{~cm}^{-2}$, for comparison. Vertical dashed line represents the angular area of the 9.1' FWHM GBT beam. (b) Distance. (c) Physical areas calculated from the $N_{\mathrm{HI}}=5 \times 10^{18} \mathrm{~cm}^{-2}$ angular diameter. (d) Galaxy density. (e) Baryonic mass using $\mathrm{M}_{\text {bary }}=1.36 \cdot \mathrm{M}_{\mathrm{HI}}+\mathrm{M}_{*}$. Vertical dashed line at the threshold given in Kereš et al. (2005). (f) Dark matter halo mass derived from the Moster et al. (2010) relation. Vertical dashed line at threshold also given in Kereš et al. (2005). (g) Rotation velocity using $\mathrm{V}_{\text {rot }}=\mathrm{W}_{20} / 2$ (h) Specific star formation rate calculated using SFR from Leroy et al. (in prep.). 
fraction as a function of galaxy parameter, such as galaxy density, baryonic mass, and rotation velocity, and others plotted in Figure 3.24. We chose these properties in order to make a comparison with theoretical predictions on the relationships between diffuse gas and cold mode accretion from the CGM.

Our diffuse fractions range from 0.05 to 0.93 of the total H I mass measured in each galaxy. Eleven of our galaxies are made up of less than $50 \%$ diffuse gas. These galaxies are generally our highest H I mass galaxies, demonstrating a perhaps intuitive correlation between mass and diffuse fraction: higher H I mass corresponds to a lower fraction of diffuse H I. This correlation can be seen in panel (f) of Figure 3.24.

In panel (a) of Figure 3.24, we demonstrate the size of the galaxies in relation to the GBT 9.1' FWHM beam area, which is illustrated by the dashed vertical line. This helps us determine if a galaxy is large enough to be resolved in the GBT beam, which tells us if the $N_{\mathrm{HI}}$ detected within the beam is representative of the real $N_{\mathrm{HI}}$ or if it is spread out over the beam, diluting the physical $N_{\mathrm{HI}}$ values of the galaxy. Each galaxy's area was determined by the number of pixels within the galaxy where column density values were greater than $1 \times 10^{19} \mathrm{~cm}^{-2}$. We note that the highest $f_{19}$ value, from ESO 300-16, corresponds to the smallest angular area. However, this galaxy is at an intermediate distance of $9.3 \mathrm{Mpc}$. At the largest distances, the GBT's beam covers the largest physical area, and could potentially be affected by beam dilution producing high values of $f_{19}$, which we do not see in panel (b). Another way to look for this bias is to plot against physical area, which we do in panel (c). There is clearly no trend in this plot, confirming a lack of bias in resolution. We draw attention to the two outlier galaxies in physical area, though: NGC 5170 and UGCA 250. These 
galaxies make up the largest distances in our sample, are the first and third largest H I disk masses in our sample, and contain less than $25 \%$ diffuse HI. We note that given these indications of a lack of bias, these two galaxies have an extremely large H I envelope. We were only able to get galaxy densities for six of our galaxies, and therefore cannot make any substantial claims with such a small sample. Although, we will note that the galaxies that we were able to obtain density information for mostly cluster at low $f_{19}$ values in panel (d).

In panel (e) we plot $f_{19}$ with baryonic mass for all but three of our galaxies. Kereš et al. (2005) show that cold mode accretion dominates at baryonic masses $\left(\mathrm{M}_{\text {bary }}=1.36 \cdot \mathrm{M}_{\mathrm{HI}}+\mathrm{M}_{*}\right)$ less than $\mathrm{M}_{\text {bary }}=10^{10.3} M_{\odot}$. We calculated the baryonic masses using our derived $M_{\mathrm{HI}}$ disk value, which was corrected by a factor of 1.36 to account for helium mass, and stellar masses given in Leroy et al. (in prep.), which had stellar masses for all but three of our sources. We do not have a large sample of masses above this threshold, but with the sample we have, this does indeed seem to complement the picture of diffuse gas from the IGM flowing directly into galaxies below this mass threshold. We show dark matter halo masses in panel (f), derived from the Moster et al. (2010) stellar-to-halo-mass relation. We see a similar trend, where larger diffuse fractions are associated with halo masses below the predicted threshold of $\mathrm{M}_{\text {halo }}=10^{11.4} M_{\odot}$ (Kereš et al., 2005), indicated by the dashed vertical line, and could potentially move that threshold closer to $\mathrm{M}_{\text {halo }}=10^{11.1} M_{\odot}$.

Rotation velocities are plotted against $f_{19}$ in panel (g) with a distinction being made at the $125 \mathrm{~km} \mathrm{~s}^{-1}$ mark. Kannappan et al. (2013) suggests that this is the threshold below which galaxies are gas-dominated. In this regime, galaxies are refu- 
eled and have larger gas fractions than those above this threshold. Once again, while we have a spread of diffuse fraction values below $v_{\text {rot }}=125 \mathrm{~km} \mathrm{~s}^{-1}$, galaxies with higher $f_{19}$ values all lie below this threshold once again supporting the picture of diffuse gas fueling galaxies. The last panel in Figure 3.24 is of specific star formation rate (sSFR) versus $f_{19}$ in panel (h). We derive sSFR, the star formation rate per unit stellar mass, using the same stellar masses mentioned above with star formation rates derived from UV+IR provided by Leroy et al. (in prep.). As star formation and galaxy mass is directly related to cold mode accretion, we want to look for trends in sSFR as it relates to both. We note in this panel that the highest diffuse fractions tend to fall at the higher end of our sSFR range, which could be contributed to by outflows from the galaxy. 
Table 3.3: Depletion timescales of the disk and halo

\begin{tabular}{|c|c|c|c|c|}
\hline Source & $\begin{array}{c}\mathrm{SFR} \\
{\left[M_{\odot} \mathrm{yr}^{-1}\right]} \\
(2)\end{array}$ & $\begin{array}{c}\log M_{\text {disk }}^{\mathrm{HI}} \\
{\left[M_{\odot}\right]} \\
(3)\end{array}$ & $\begin{array}{c}\tau_{d i s k}^{g a s} \\
{[\mathrm{Gyr}]} \\
(4)\end{array}$ & $(5)$ \\
\hline ESO300-G014 & 0.063 & 9.39 & 52.91 & 0.438 \\
\hline ESO300-G016 & $\ldots$ & 8.27 & $\ldots$ & 0.93 \\
\hline ESO302-G014 & 0.038 & 8.92 & 29.75 & 0.639 \\
\hline ESO357-G007 & 0.041 & 9.32 & 69.75 & 0.604 \\
\hline KK98-195 & $\ldots$ & 7.98 & $\ldots$ & 0.718 \\
\hline KKS2000-23 & $\ldots$ & 9.0 & $\ldots$ & 0.715 \\
\hline NGC1371 & 0.427 & 10.22 & 52.91 & 0.165 \\
\hline NGC1592 & 0.079 & 8.63 & 7.3 & 0.83 \\
\hline NGC1744 & 0.195 & 9.88 & 52.91 & 0.088 \\
\hline NGC3511 & 0.447 & 9.82 & 20.12 & 0.213 \\
\hline NGC5068 & 0.288 & 9.6 & 18.77 & 0.068 \\
\hline NGC5170 & 0.589 & 10.56 & 83.86 & 0.142 \\
\hline NGC5253 & 0.525 & 8.36 & 0.59 & 0.303 \\
\hline NGC7424 & 0.372 & 10.38 & 87.81 & 0.05 \\
\hline UGCA015 & 0.001 & 7.39 & 45.03 & 0.826 \\
\hline UGCA250 & 0.603 & 10.36 & 51.71 & 0.22 \\
\hline UGCA307 & 0.031 & 9.02 & 46.08 & 0.434 \\
\hline UGCA320 & 0.021 & 9.54 & 225.7 & 0.175 \\
\hline
\end{tabular}

Table 3.3: Star formation rates obtained from Leroy et al. (in prep). Column 3 lists the H I mass of the disk. Column 4 lists the gas depletion timescales for the disk gas, including a correction for Helium mass.

\subsubsection{Depletion timescales}

One method of determining whether nearby galaxies contain enough gas to continue to fuel star formation is to calculate the depletion timescale of the galaxy, given the gas mass and the SFR. This timescale determines how long it will take the 
galaxy to use up all of its gas if all of this gas is converted into stars. Our depletion timescales are calculated with:

$$
\tau_{d i s k}^{g a s}=\frac{M_{d i s k}^{g a s}}{S F R},
$$

where $\tau_{\text {disk }}^{\text {gas }}$ is the depletion timescale given the disk gas alone, and $M_{\text {disk }}^{\text {gas }}$ is our measure H I disk mass corrected for helium with a factor of 1.36. These timescales, listed in Table 3.3, tell us that these galaxies currently have enough gas to sustain star formation for well over a Hubble time, consistent with the H I depletion timescales found by both Bigiel et al. (2010) and Roychowdhury et al. (2014), with the exception of NGC 1592 and NGC 5253. The $f_{19}$ values for these two galaxies are 0.83 and 0.303 , respectively. Their H I masses are low, but similar. We did note a potential source of accretion in the NGC 5253 map, however. If this source is undergoing ongoing accretion, this could explain the lack of an established reservoir of diffuse H I, as well as its shorter depletion timescale.

\subsection{Summary}

We analyzed $21 \mathrm{~cm}$ data from GBT observations of a sub-sample of $18 \mathrm{MHON}-$ GOOSE galaxies to search for diffuse, low column density H I in the CGM of these galaxies. We reached a mean $1 \sigma$ column density sensitivity of $N_{\mathrm{HI}}=1.3 \times 10^{17} \mathrm{~cm}^{-2}$ per channel, and a $3 \sigma$ column density of $N_{\mathrm{HI}}=1.3 \times 10^{18} \mathrm{~cm}^{-2}$ over a linewidth of 20 $\mathrm{km} \mathrm{s}^{-1}$.

1. When comparing our values for the total cumulative H I mass in the sub-maps to the H I measured in the disk of each galaxy, we found six that had an increase 
in the total amount of HI. ESO 300-16, ESO 302-14, KK98-195, KKS2000-23, NGC 1592, and NGC 5170 contained 2-45\% more extraplanar H I.

2. We compared the amount of diffuse H I, as defined by the amount of H I below $N_{\mathrm{HI}}=10^{19} \mathrm{~cm}^{-2}$, in each galaxy to the total H I mass. The percentage of diffuse gas in the galaxies ranged from $\sim 5 \%$ to $\sim 93 \%$. Over half of our galaxies contained less than $50 \%$ diffuse H I.

3. Most of the galaxies in our sample showed a relatively steady average $N_{\mathrm{HI}}$ level at radii beyond the disk. These levels were typically between $3 \sigma$ and $5 \sigma$, with seven galaxies (KKS2000-23, NGC 1744, NGC 3511, NGC 5170, NGC 7424, UGCA250, and UGCA320) displaying average $N_{\mathrm{HI}}$ levels above $5 \sigma$ at these radii.

4. We compared the diffuse fraction of each source with various environmental properties of galaxies. We emphasize that the diffuse fraction that we measured supports current theories of cold mode accretion of diffuse gas onto galaxies when looking at baryonic mass, dark matter halo mass, and rotation velocities. This is potentially further supported by the specific star formation rates calculated in these galaxies, where higher diffuse fractions were found associated with higher sSFR.

The high resolution MHONGOOSE survey with MeerKAT will provide more insight into the origins of low column density gas in the CGM of these galaxies. Galaxies with diffuse fractions supporting cold mode accretion models in our sample will be better understood with higher resolution data. With this single-dish counterpart 
data to the MeerKAT interferometer data, we will have a complete census of the H I in each of these galaxies at all angular scales. 


\section{Chapter 4}

\section{Probing the Full Virial Volume of IMAGINE Galaxies}

\subsection{Abstract}

We present an early data release of the Imaging Galaxies Intergalactic and Nearby Environment (IMAGINE) survey with the Parkes Radio Telescope. We mapped a region around 28 nearby galaxies that extends out to or beyond the virial radius of each galaxy, encompassing their entire dark matter halos. We present 18 of those galaxies here. Within this region, we reach $1 \sigma$ column density sensitivities of $N_{\mathrm{HI}}=10^{16.15-16.62} \mathrm{~cm}^{-2}$ per channel with a mean rms noise of $6.8 \mathrm{mJy}$, and $\mathrm{HI}$ mass sensitivities down to $M_{\mathrm{HI}}=10^{4.87} M_{\odot}$. Mapping the full virial volume enabled us to quantify the amount of $\mathrm{H}$ I mass detected in the circumgalactic medium of these galaxies. We found that seven of our galaxies contain more H I gas in their CGM than in their disk. We compared the fraction of diffuse H I from each map to a variety of galaxy and environmental properties and did not find any strong correlations. We also derived the gas depletion timescales using the H I corrected for helium. We calculated these timescales two ways: using only the amount in the disk, and using the total H I throughout the halo. When accounting for the disk gas only, we found that eight of our galaxies contained enough gas to sustain star formation for over a Hubble time, and when accounting for all of the H I throughout the halo, we found that 10 of our galaxies contained enough gas to sustain star formation for over a Hubble time. 
This work will soon be submitted for publication as Sardone et al. (2019)

Contributing authors: D.J. Pisano, Attila Popping, Jongwhan Rhee, Lister Staveley-Smith, and Dane Kleiner. 


\subsection{Introduction}

The rate at which galaxies form stars has decreased over the last several billion years (Madau \& Dickinson, 2014), while the amount of neutral hydrogen, the fuel for that star formation, has remained nearly constant over the same time scale (e.g., Noterdaeme et al., 2012; Crighton et al., 2015). It can be concluded then that galaxies must be getting their hydrogen from their environment, specifically the inter- and circum-galactic mediums (IGM and CGM). As gas from the IGM falls onto galaxies, it is sometimes accreted onto the galaxy without heating up, through a process called cold mode accretion (Kereš et al., 2005). This gas falls along a network of dense filaments making up the cosmic web (Bond et al., 1996), which connects all galaxies in the universe.

Before gas falls onto a galaxy from the IGM, it must first enter the galaxy's CGM. Detecting infalling gas has proved difficult as column density levels of this gas are very low. Tumlinson et al. (2013) find that gas associated with the galaxy, seen in absorption, is typically found at column density levels of $N_{\mathrm{HI}} \geq 10^{16} \mathrm{~cm}^{-2}$, while most H I surveys reach column density levels of $N_{\mathrm{HI}} \geq 10^{18} \mathrm{~cm}^{-2}$ (HIPASS; Koribalski et al. (2004), ALFALFA; Haynes et al. (2011)). Some H I surveys have specifically targeted gas throughout the CGM of galaxies, such as the HALOGAS survey (Heald et al., 2011) and the GBT survey of HALOGAS galaxies (Pingel et al., 2018), yet will only reach H I column densities of a few times $N_{\mathrm{HI}}=10^{19} \mathrm{~cm}^{-2}$ for the former, and $N_{\mathrm{HI}} \geq 10^{18} \mathrm{~cm}^{-2}$ for the latter. To date there have been no large surveys of nearby galaxies targeting HI emission in the CGM with the sensitivity to detect column 
densities below $N_{\mathrm{HI}}=10^{18} \mathrm{~cm}^{-2}$. Braun \& Thilker (2004) mapped a large swath of sky centered on M31 down to similar sensitivities, but do not attempt to characterize the CGM around detected galaxies.

In this chapter, we introduce the full IMAGINE survey in Section 4.3. Detailed information on our observations and data reduction methods are laid out in Section 4.4. We present the analysis of this data in Section 4.5. Our results can be found in Section 4.6, and our discussion of these results in Section 4.7. This work is summarized in Section 4.8.

\subsection{The IMAGINE Survey and Galaxy Sample}

The Imaging Galaxies Intergalactic and Nearby Environment (IMAGINE ${ }^{1}$ ) survey is an investigation of the neutral hydrogen around 28 nearby spiral galaxies, probing the $\mathrm{H}$ I at extremely low column densities with the Australia Telescope Compact Array (ATCA) and the Parkes Radio Telescope. As spiral galaxies display active star formation, they are the prime targets for detecting the accretion of gas onto galaxies. $\mathrm{HI}$ is the best tracer for this accretion as atomic gas flows directly into galaxies from the IGM and CGM, and eventually compresses into molecular hydrogen, $H_{2}$, the fuel from which galaxies form stars.

Galaxies were selected based on a number of criteria that do not include prior knowledge of the HI content, in order to obtain an unbiased sample. Each spiral galaxy has a declination below $-20^{\circ}$ to optimize the shape of the synthesized beam of the ATCA. Galaxies were also chosen based on their optical isophotal diameter at 
the 25 th magnitude, $D_{25}$, in the B-band. With a lower limit of $5^{\prime}$ and an upper limit of $20^{\prime}$, each galaxy will be at least somewhat resolved at the lowest angular resolution, while any extended $\mathrm{H}$ I gas from those at the higher end will still be detectable within ATCA's Field of View, where the full width at half maximum (FWHM) of the primary beam is $34^{\prime}$ at $1.4 \mathrm{GHz}$. Galaxies within $20 \mathrm{Mpc}$ were also chosen for the potential to resolve gas clumps and filaments. The resulting selection contains galaxies in a range of environments, from isolated to groups, H I masses over several orders of magnitude, optical diameters, and $B$-magnitudes. These basic properties of the galaxies in our sample can be seen in Table 4.1.

We use both single-dish and interferometer telescopes to obtain a large range of angular scales. The ATCA is used in all compact configurations, achieving angular resolutions between $1^{\prime}$ and $2.5^{\prime}$. With ATCA, the 28 galaxies will be observed for 96 hours each, extending to physical radii of $\mathrm{r} \leq 150 \mathrm{kpc}$. These large integration times will enable us to reach $3 \sigma$ detection sensitivities of $N_{\mathrm{HI}} \approx 7 \times 10^{17} \mathrm{~cm}^{-2}$ over a $20 \mathrm{~km} \mathrm{~s}^{-1}$ linewidth, lower than any previous survey.

\footnotetext{
${ }^{1}$ http://www.imagine-survey.org/
} 
Table 4.1: Sample of IMAGINE Galaxies

\begin{tabular}{|c|c|c|c|c|c|}
\hline Source & $\begin{array}{c}\text { R.A. }(2000.0) \\
{[\mathrm{deg}]} \\
(2)\end{array}$ & $\begin{array}{c}\text { Decl. }(2000.0) \\
\text { [deg] } \\
(3)\end{array}$ & $\begin{array}{c}\mathrm{D} \\
{[\mathrm{Mpc}]} \\
(4)\end{array}$ & $\begin{array}{c}\text { incl. } \\
{[\mathrm{deg}]} \\
(5)\end{array}$ & $\begin{array}{r}D_{25} \\
{\left[{ }^{\prime}\right]} \\
(6)\end{array}$ \\
\hline ESO138-10 & 254.7622 & -60.2160 & 14.7 & 49.2 & 5.5 \\
\hline ESO154-23 & 44.2099 & -54.5714 & 5.9 & 90.0 & 8.13 \\
\hline ESO209-9 & 119.5642 & -49.8542 & 14.05 & 90.0 & 6.46 \\
\hline ESO214-17 & 160.0780 & -48.5696 & 13.1 & 36.0 & 5.25 \\
\hline ESO270-17 & 203.6970 & -45.5475 & 7.6 & 90.0 & 11.48 \\
\hline ESO274-1 & 228.5576 & -46.8079 & 4.0 & 90.0 & 9.77 \\
\hline IC5201 & 335.2393 & -46.0358 & 12.0 & 66.6 & 6.76 \\
\hline NGC1433 & 55.5064 & -47.2220 & 9.9 & 67.4 & 6.17 \\
\hline NGC1512 & 60.9761 & -43.3488 & 10.4 & 68.3 & 8.51 \\
\hline NGC1744 & 74.9908 & -26.0222 & 8.6 & 69.9 & 5.25 \\
\hline NGC1792 & 76.3102 & -37.9807 & 11.5 & 63.1 & 5.62 \\
\hline NGC2188 & 92.5397 & -34.1062 & 7.6 & 90.0 & 5.37 \\
\hline NGC24 & 2.4855 & -24.9631 & 8.4 & 70.1 & 6.17 \\
\hline NGC2835 & 139.4704 & -22.3546 & 8.7 & 56.2 & 6.46 \\
\hline NGC2997 & 146.4116 & -31.1910 & 11.0 & 53.7 & 10.23 \\
\hline NGC3109 & 150.7786 & -26.1595 & 1.8 & 90.0 & 15.85 \\
\hline NGC3137 & 152.2811 & -29.0643 & 15.9 & 47.2 & 5.5 \\
\hline NGC3175 & 153.6754 & -28.8720 & 14.3 & 76.2 & 5.25 \\
\hline NGC45 & 3.5166 & -23.1820 & 7.3 & 51.5 & 6.17 \\
\hline NGC625 & 23.7692 & -41.4361 & 4.6 & 90.0 & 6.61 \\
\hline NGC7424 & 344.3265 & -41.0705 & 11.5 & 59.0 & 5.01 \\
\hline NGC7793 & 359.4576 & -32.5910 & 3.4 & 63.5 & 10.47 \\
\hline
\end{tabular}

Table 4.1: Positions listed in Columns 2-3 and distances in 4 gathered from NED. Values from Columns 5-6 taken from HyperLEDA: http://leda.univ-lyon1.fr.

In addition to our ATCA observations, we are also using the 64-meter Parkes Radio Telescope, which has an angular resolution of $14.4^{\prime}$. Large single-dish telescopes are superior in detecting diffuse, large-scale structure, which is the focus of these Parkes observations. We chose integration times for each galaxy to be long enough 
Table 4.2: Stellar and Halo Masses - two derivations

\begin{tabular}{lcccc}
\hline \hline Source & $\log M_{*}$ & $\log M_{*}$ & $\log M_{\text {halo }}$ & $\log M_{\text {halo }}$ \\
Method & {$\left[M_{\odot}\right]$} & {$\left[M_{\odot}\right]$} & {$\left[M_{\odot}\right]$} & {$\left[M_{\odot}\right]$} \\
\hline ESO138-10 & 10.88 & 10.14 & 12.5 & 11.7 \\
ESO154-23 & 8.93 & 8.48 & 11.1 & 10.9 \\
ESO209-9 & 10.84 & 9.82 & 12.5 & 11.5 \\
ESO214-17 & 8.92 & 8.92 & 11.1 & 11.1 \\
IC5201 & 9.98 & 9.81 & 11.6 & 11.5 \\
NGC1433 & 10.55 & 10.64 & 12.1 & 12.2 \\
NGC1512 & 10.5 & 10.49 & 12.0 & 12.0 \\
NGC1744 & 9.75 & 9.19 & 11.5 & 11.2 \\
NGC1792 & 10.45 & 10.26 & 12.0 & 11.8 \\
NGC2188 & 9.42 & 9.16 & 11.3 & 11.2 \\
NGC24 & 9.79 & 8.89 & 11.5 & 11.0 \\
NGC2835 & 10.05 & 9.64 & 11.7 & 11.4 \\
NGC3137 & 10.12 & 9.7 & 11.7 & 11.4 \\
NGC3175 & 10.68 & 10.14 & 12.2 & 11.7 \\
NGC45 & 9.68 & 9.36 & 11.5 & 11.3 \\
NGC625 & 9.18 & 8.53 & 11.2 & 10.9 \\
NGC7424 & 10.08 & 9.31 & 11.7 & 11.2 \\
NGC7793 & 9.56 & 9.21 & 11.4 & 11.2 \\
\hline \hline
\end{tabular}

Table 4.2: Comparison in values of stellar mass and halo mass using two methods. "B\&dJ" represents the method of using galaxy colors to derive stellar masses from Bell \& de Jong (2001), while "L" represents the stellar masses obtained from Leroy et al. (2019, in prep.). We calculated the dark matter halo mass from the Moster et al. (2010) stellar-mass-halo-mass relation, represented by "M", using the two types of stellar masses as described above. We find that the median halo masses derived from the Bell \& de Jong (2001) stellar masses are 1.8 times larger than those by Leroy et al. (2019, in prep.).

to allow us to reach $1 \sigma$ column density sensitivities of $N_{\mathrm{HI}} \sim 1.5 \times 10^{16} \mathrm{~cm}^{-2}$ per $2.6 \mathrm{~km} \mathrm{~s}^{-1}$ channel, or $3 \sigma \mathrm{HI}$ column density levels of $N_{\mathrm{HI}} \sim 3 \times 10^{17} \mathrm{~cm}^{-2}$ over a $20 \mathrm{~km} \mathrm{~s}^{-1}$ linewidth, making this one of the deepest H I emission surveys to date. Additionally, while the ATCA maps produced will provide extremely sensitive, detailed 
images of the region close to each galaxy, each map made with Parkes will extend out to or beyond the virial radius, $R_{v i r}$, encompassing the CGM of each galaxy, where $R_{v i r}$ ranges from $174<R_{v i r}<806 \mathrm{kpc}$.

The virial radius is defined to be the radius at which a volume containing an overdensity of 200, as suggested by simulations, times the critical density of the Universe is enclosed. Interior to this radius the material is virialized, and exterior to this radius mass is still collapsing. As such, it is sometimes referred to as $R_{200}$. We calculate the virial radius using the virial mass, which we define as the total mass of the galaxy's dark matter halo. From the typical mass equation $\left(M=4 / 3 \pi r^{3} \rho\right)$, we can solve for the radius,

$$
R_{v i r}=\left(\frac{3 M_{v i r}}{4 \pi 200 \rho_{c}}\right)^{1 / 3}
$$

where the density, $\rho_{c}=4.05 \times 10^{10} M_{\odot} / \mathrm{Mpc}^{3}$ using $H_{0}=73 \mathrm{~km} \mathrm{~s}^{-1} \mathrm{Mpc}^{-1}$, is the critical density of the Universe. With this equation, we calculated the extent of the region to map around each galaxy. To determine the mapping area for each galaxy, we used the Moster et al. (2010) stellar-mass-halo-mass relation to derive the halo mass, or $M_{v i r}$. Using this relation requires a stellar mass, which we calculated using the Bell \& de Jong (2001) relation between stellar mass to light ratio and galaxy color, where galaxy colors were taken from LEDA. From our derived $M_{v i r}$, or halo mass, we set our map sizes, which range from a minimum of $2^{\circ} \times 2^{\circ}$ up to $10^{\circ} \times 10^{\circ}$ for our nearest source. Since our original calculations, we obtained new stellar masses for each IMAGINE galaxy (Leroy et al., in prep.), which were derived from FUV and 
infrared emission. As both FUV and infrared are excellent tracers of young stars, this is a more reliable estimate of the stellar masses and star formation rates than our previous calculations. While we used our stellar and halo mass calculations to set the boundaries of our maps, we perform our subsequent analysis using the newly obtained stellar masses. We have tabulated stellar and halo masses we derived along with those obtained from Leroy et al., (in prep.) in Table 4.2, where the derived values are consistently higher than those from the FUV and infrared data. Histograms with some of the galaxy properties of all 28 galaxies in our sample can be seen in Figure 4.1. With Parkes-IMAGINE, we are producing the first survey mapping the entire CGM around spiral galaxies down to column density sensitivities of $N_{\mathrm{HI}} \sim 10^{16-17} \mathrm{~cm}^{-2}$. In the following sections, we proceed with a subsample of the IMAGINE galaxies which we have obtained complete maps for.

\subsection{Observations and Data Reduction}

The data presented in this chapter was obtained as a portion of project P951 during Parkes observation semesters 2017APR, 2017OCT, and 2018OCT in which we observed for a total of 218 of our requested 394 hours. With this time, we were able to complete observations and analysis of 18 of the 28 sources, which will be presented here.

We used the 20-cm Parkes multibeam receiver (Staveley-Smith et al., 1996) to observe the 21-cm line of neutral hydrogen. Using the multibeam receiver expedites mapping due to the multiple beams formed on the sky. Each of the 13 beams produce 
Figure 4.1: Full IMAGINE Sample
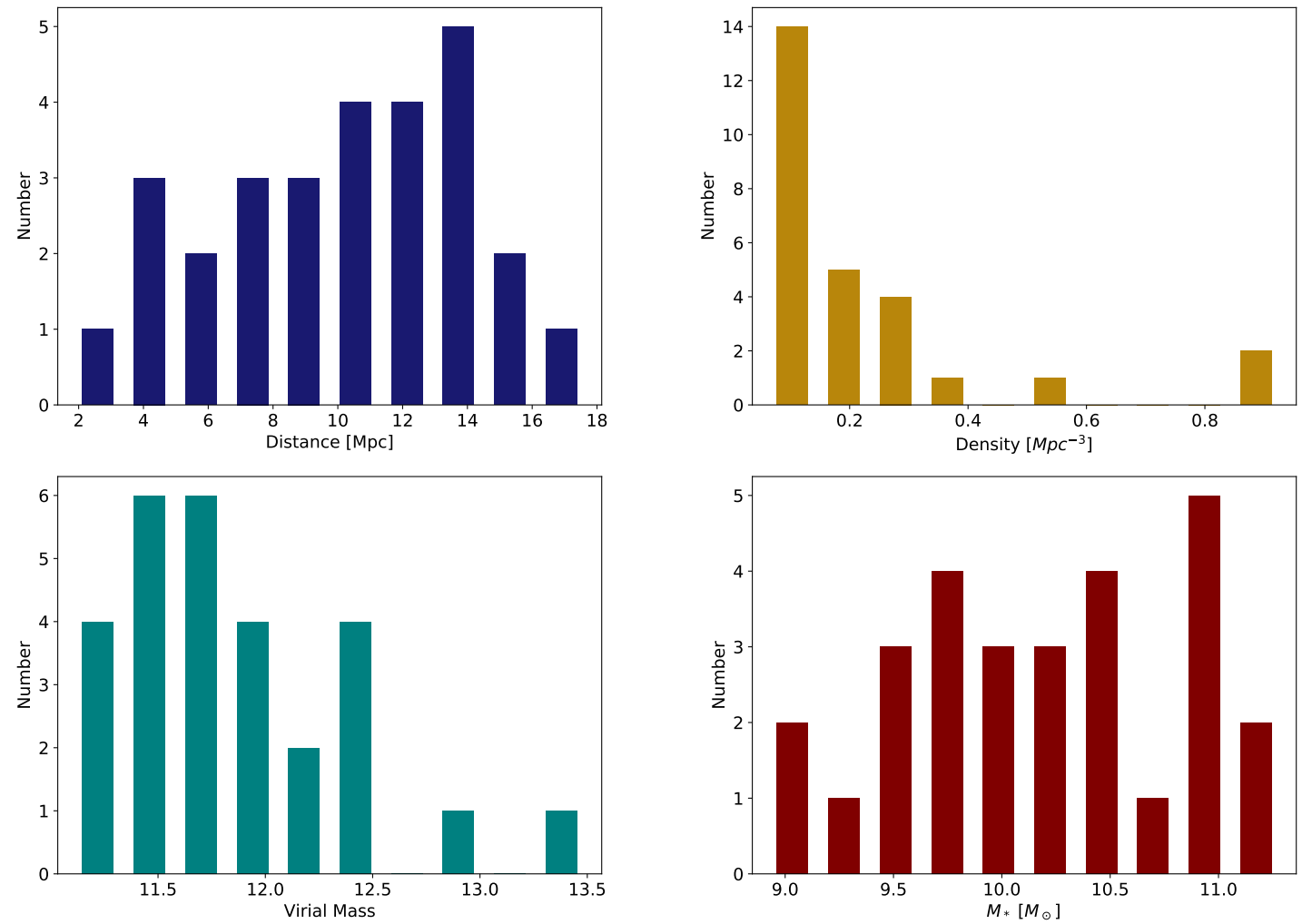

Notes. The full IMAGINE sample is made up of galaxies with a range of properties displayed here. Clockwise from top left: Distances taken from NED, galaxy density per $\mathrm{Mpc}^{3}$ as listed in the Nearby Galaxies Catalog (Tully, 1988), virial mass derived using the Moster et al. (2010) relation, and stellar masses calculated from Bell \& de Jong (2001). 
a 14.4' angular resolution at full width at half power (FWHP), forming a hexagonal pattern on the sky. We also used the HIPSR backend (Price et al., 2016), which produces high spectral resolution modes, which are ideal for observing H I. We used HIPSR in the 16384-channel, $200 \mathrm{MHz}$ bandwidth mode, producing the high spectral resolution $(12.2 \mathrm{kHz}$ or $2.6 \mathrm{~km} / \mathrm{s}$ channels) needed to resolve structure that would otherwise not be seen with coarser channels. We filled in each map by scanning in a basket-weave pattern, half of the scans in right ascension and the other half in declination. Scanning in this way produces maps with uniform coverage of noise properties, which stay relatively even apart from the edges of the maps. For this reason, we chose to map an area slightly larger than the derived virial radius in order to maintain uniform noise throughout our targeted region. Some of our maps extend well beyond the virial radius due to our minimum mapping size of $2^{\circ} \times 2^{\circ}$. We observed with 2 second integration cycles, with a total integration time needed to map to our desired sensitivity between 5-91 hours. We calculated this integration time by first determining our desired column density sensitivity with

$$
N_{\mathrm{HI}}=1.822 \times 10^{18}\left(\frac{T_{\mathrm{B}}}{\mathrm{K}}\right)\left(\frac{d v}{\mathrm{~km} \mathrm{~s}^{-1}}\right) \mathrm{cm}^{-2},
$$

which becomes

$$
\sigma_{N_{\mathrm{HI}}}=1.822 \times 10^{18} \cdot \sigma_{T_{\mathrm{B}}} \cdot \sqrt{W \cdot \Delta v} \mathrm{~cm}^{-2}
$$

where $\sigma_{T_{\mathrm{B}}}$ is in $\mathrm{K}, W$ is the linewidth of the $\mathrm{HI}$ spectrum in $\mathrm{km} \mathrm{s}^{-1}$, and $\Delta v$ is the velocity resolution of the data, also in $\mathrm{kms}^{-1}$. Using our desired $5 \sigma$ detection 
sensitivity of $N_{\mathrm{HI}} \sim 2.5 \times 10^{17} \mathrm{~cm}^{-2}$, we can solve for our brightness temperature noise sensitivity:

$$
\sigma_{T_{\mathrm{B}}}=\frac{2.5 \times 10^{17}}{5 \cdot 1.822 \times 10^{18} \sqrt{W \cdot \Delta v}}=\frac{0.0274}{\sqrt{W \cdot \Delta v}} \mathrm{~K} .
$$

This gives us a brightness temperature in $\mathrm{K}$, which we can convert to units of Jy using the $\mathrm{G}=1.1 \mathrm{Jy} / \mathrm{K}$ gain of the Parkes telescope with $\sigma_{J y}=\sigma_{T_{\mathrm{B}}} \cdot G$. From the radiometer equation, we can calculate the effective integration time in seconds, $t_{\text {eff }}$ for each map:

$$
t_{\mathrm{eff}}=\frac{\mathrm{T}_{\mathrm{sys}}^{2}}{\mathrm{~N}_{\mathrm{pol}} \cdot \Delta v \cdot \sigma_{T_{\mathrm{B}}}^{2}} \mathrm{sec},
$$

where we use a system temperature, $\mathrm{T}_{\mathrm{sys}}$, of $28 \mathrm{~K}$, and the number of polarizations, $\mathrm{N}_{\text {pol }}$, is 2 .

We receive the data in the form of SDFITS files, which can be read into the LIVEDATA $^{4}$ and GRIDZILLA ${ }^{4}$ programs for reduction. We used LIVEDATA to smooth and bandpass-correct the data. Baselines were fitted with a robust first, second, or third-order polynomial, excluding points outside of three times the standard deviation for the fit. After baseline correction, the data was smoothed using a Hanning filter to best remove Gibbs ringing from Galactic emission. Following this process, we gridded the data with GRIDZILLA. This software reads in the output files from LIVEDATA, and combines and grids them into a new data cube. The data cubes consist of pixels with sizes of $4^{\prime} \times 4^{\prime}$. Contributing to the value of each pixel is a set of spectra whose weights and maximum radius has been set to a median weighting function and a cutoff radius of $6^{\prime}$, as is recommended in Barnes et al. (2001). We selected a $12^{\prime}$ top-hat kernel for spatial smoothing. The finished product is a data cube with two spatial axes in right 
ascension and declination, with a third axis in velocity spanning the range from -500 to $2000 \mathrm{~km} / \mathrm{s}$, covering all emission associated with these galaxies.

\subsection{Analysis}

Our processed data cubes were used to analyze the H I properties of each central galaxy. From these cubes, we can make a number of measurements, among which is the total integrated flux from each source, its linewidth and systemic velocity, and the rms noise in the cube. We can use these measurements to derive galaxy properties such as H I masses, H I diameters, and total dynamical masses of each galaxy. With cubes of such a large physical extent, we can also evaluate the environment around each galaxy and quantify the H I content throughout the dark matter halo of these galaxies. We will discuss these analysis processes in the sections below.

\subsubsection{H i Measurements and Galaxy Properties}

We measured H I properties of the central galaxies in each cube using the MIRIAD task MBSPECT. This task performs a fit over a specified spatial and spectral region, applying either a first, second, or third-order polynomial, which we specify, to the baseline, which is then subtracted for the the final spectra. With this measurement, we get the output values for the total integrated flux, rms noise, systemic velocities, and the linewidths at $20 \%$ and $50 \%$ maximum, among others. These measured H I values can be found in Table 4.3 for each of our sources.

\footnotetext{
${ }^{4}$ https://www.atnf.csiro.au/computing/software/
} 
Table 4.3: H I Measurements and Derived Properties of IMAGINE Galaxies

\begin{tabular}{|c|c|c|c|c|c|c|c|c|c|c|c|}
\hline Source & $\begin{array}{c}\sigma_{r m s} \\
{[\mathrm{Jy}]} \\
(2)\end{array}$ & $\begin{array}{c}S_{\mathrm{HI}} \\
{\left[\mathrm{Jy} \mathrm{km} \mathrm{s}^{-1}\right]} \\
(3)\end{array}$ & $\begin{array}{c}\mathrm{V}_{\text {sys }} \\
{\left[\mathrm{km} \mathrm{s}^{-1}\right]} \\
(4)\end{array}$ & $\begin{array}{c}\mathrm{W}_{50} \\
{\left[\mathrm{~km} \mathrm{~s}^{-1}\right]} \\
(5)\end{array}$ & $\begin{array}{c}\mathrm{W}_{20} \\
{\left[\mathrm{~km} \mathrm{~s}^{-1}\right]} \\
(6)\end{array}$ & $\begin{array}{c}\log _{10} N_{\mathrm{HI}} 1 \sigma \\
{\left[\mathrm{cm}^{-2}\right]} \\
(7)\end{array}$ & $\begin{array}{c}\log _{10} N_{\text {HI } 5 \sigma} \\
{\left[\mathrm{cm}^{-2}\right]} \\
(8)\end{array}$ & $\begin{array}{c}\log _{10} \mathrm{M}_{1 \sigma} \\
{\left[M_{\odot}\right]} \\
(9)\end{array}$ & $\begin{array}{c}\log _{10} \mathrm{M}_{\mathrm{HI}} \\
{\left[M_{\odot}\right]} \\
(10)\end{array}$ & $\begin{array}{c}\log _{10} \mathrm{M}_{\mathrm{dyn}} \\
{\left[M_{\odot}\right]} \\
(11)\end{array}$ & (12) \\
\hline ESO138-10 & 0.0055 & 146.7 & 1167.4 & 209.6 & 227.3 & 16.22 & 17.58 & 5.86 & 9.87 & 11.02 & 0.072 \\
\hline ESO154-23 & 0.0047 & 120.4 & 562.4 & 121.9 & 140.6 & 16.15 & 17.51 & 5.0 & 9.0 & 10.13 & 0.073 \\
\hline ESO209-9 & 0.0125 & 96.37 & 1146.8 & 308.5 & 334.5 & 16.57 & 17.94 & 6.18 & 9.65 & 11.16 & 0.031 \\
\hline ESO214-17 & 0.0048 & 92.43 & 1071.6 & 30.8 & 46.2 & 16.16 & 17.52 & 5.7 & 9.57 & 9.78 & 0.614 \\
\hline ESO270-17 & 0.015 & 217.7 & 806.5 & 137.4 & 155.1 & 16.65 & 18.02 & 5.72 & 9.47 & 10.48 & 0.099 \\
\hline ESO274-1 & 0.0164 & 143.0 & 494.4 & 166.6 & 178.9 & 16.69 & 18.06 & 5.21 & 8.73 & 10.25 & 0.03 \\
\hline IC5201 & 0.007 & 141.8 & 937.7 & 188.4 & 205.5 & 16.32 & 17.69 & 5.79 & 9.68 & 10.77 & 0.083 \\
\hline NGC1433 & 0.0049 & 25.21 & 1061.9 & 167.8 & 185.1 & 16.17 & 17.53 & 5.47 & 8.77 & 10.55 & 0.017 \\
\hline NGC1512 & 0.0113 & 193.9 & 893.5 & 233.4 & 267.1 & 16.53 & 17.9 & 5.87 & 9.69 & 11.02 & 0.047 \\
\hline NGC1744 & 0.005 & 124.8 & 734.6 & 191.8 & 208.1 & 16.18 & 17.54 & 5.35 & 9.34 & 10.5 & 0.068 \\
\hline NGC1792 & 0.0049 & 37.02 & 1227.3 & 293.0 & 324.4 & 16.17 & 17.53 & 5.6 & 9.06 & 11.09 & 0.009 \\
\hline NGC2188 & 0.0054 & 30.88 & 732.6 & 110.0 & 146.5 & 16.21 & 17.57 & 5.31 & 8.66 & 10.12 & 0.035 \\
\hline NGC24 & 0.014 & 50.41 & 571.4 & 204.9 & 218.9 & 16.62 & 17.99 & 5.78 & 8.92 & 10.6 & 0.021 \\
\hline NGC2835 & 0.0052 & 122.4 & 867.5 & 191.6 & 211.3 & 16.19 & 17.56 & 5.38 & 9.34 & 10.72 & 0.042 \\
\hline NGC2997 & 0.0191 & 416.6 & 1105.1 & 247.8 & 273.9 & 16.76 & 18.12 & 6.15 & 10.08 & 11.27 & 0.064 \\
\hline NGC3109 & 0.0487 & 2384.0 & 371.6 & 86.0 & 116.8 & 17.16 & 18.53 & 4.98 & 9.26 & 9.74 & 0.33 \\
\hline NGC3137 & 0.0066 & 100.7 & 1101.5 & 250.9 & 266.2 & 16.3 & 17.66 & 6.01 & 9.78 & 11.22 & 0.036 \\
\hline NGC3175 & 0.0058 & 9.633 & 1110.5 & 298.3 & 392.7 & 16.24 & 17.61 & 5.86 & 8.67 & 11.25 & 0.003 \\
\hline NGC45 & 0.0103 & 222.3 & 458.9 & 166.7 & 182.9 & 16.49 & 17.86 & 5.53 & 9.45 & 10.55 & 0.079 \\
\hline NGC625 & 0.0057 & 37.12 & 405.0 & 71.8 & 102.0 & 16.23 & 17.6 & 4.87 & 8.27 & 9.66 & 0.041 \\
\hline NGC7424 & 0.005 & 209.2 & 957.2 & 151.9 & 170.0 & 16.18 & 17.54 & 5.61 & 9.81 & 10.51 & 0.201 \\
\hline
\end{tabular}

Continued on next page 
Table 4.3 - Continued from previous page

\begin{tabular}{lcccccccccccc}
\hline Source & $\sigma_{\text {rms }}$ & $S_{\mathrm{H} \mathrm{I}}$ & $\mathrm{V}_{\text {sys }}$ & $\mathrm{W}_{50}$ & $\mathrm{~W}_{20}$ & $\log _{10} N_{\mathrm{HI} 1 \sigma}$ & $\log _{10} N_{\mathrm{HI}} 5 \sigma$ & $\log _{10} \mathrm{M}_{1 \sigma}$ & $\log _{10} \mathrm{M}_{\mathrm{H} \mathrm{I}}$ & $\log _{10} \mathrm{M}_{\mathrm{dyn}}$ & $f_{\mathrm{HI}}$ \\
& {$[\mathrm{Jy}]$} & {$\left[\mathrm{Jy} \mathrm{km} \mathrm{s}^{-1}\right]$} & {$\left[\mathrm{km} \mathrm{s}^{-1}\right]$} & {$\left[\mathrm{km} \mathrm{s}^{-1}\right]$} & {$\left[\mathrm{km} \mathrm{s}^{-1}\right]$} & {$\left[\mathrm{cm}^{-2}\right]$} & {$\left[\mathrm{cm}^{-2}\right]$} & {$\left[M_{\odot}\right]$} & {$\left[M_{\odot}\right]$} & {$\left[M_{\odot}\right]$} \\
$(1)$ & $(2)$ & $(3)$ & $(4)$ & $(5)$ & $(6)$ & $(7)$ & $(8)$ & $(9)$ & $(10)$ & $(11)$ & $(12)$ \\
\hline NGC7793 & 0.0051 & 255.0 & 245.5 & 173.9 & 190.9 & 16.19 & 17.55 & 4.56 & 8.84 & 10.36 & 0.03 \\
\hline \hline
\end{tabular}

Table 4.3: (1) Source name. (2) measured rms noise. (3) Total integrated flux. (4) Systemic velocity. (5) Linewidth at 50\% maximum. (6) Linewidth at $20 \%$ maximum. (7) $1 \sigma_{r m s}$ column density sensitivity per channel. (8) $3 \sigma_{\text {rms }}$ column density level over a $20 \mathrm{~km} \mathrm{~s}^{-1}$ linewidth. (9) H I mass sensitivity. (10) H I mass. (11) Dynamical mass. (12) Neutral gas fraction, $f_{\mathrm{HI}}=$ $M_{\mathrm{HI}} / M_{d y n}$. 
From the values listed above, we can derive several galaxy properties. We derive H I masses for each source, assuming the H I is optically thin, with:

$$
M_{\mathrm{HI}}=2.36 \times 10^{5} D^{2} \int_{v_{1}}^{v_{2}} S(v) d v \mathrm{M}_{\odot} .
$$

Here, the distance, $D$, is in Mpc, and the total integrated flux value in units of Jy $\mathrm{km} / \mathrm{s}$ comes from integrating over the velocity range of the galaxy's H I profile. Our measured rms noise, $\sigma_{r m s}$, gives us our actual H I column density sensitivity, which we can use to calculate both the $1 \sigma$ sensitivity per spectral channel, and the $5 \sigma$ detection sensitivity over a specified H I linewidth. For the former, we use,

$$
\sigma_{N_{\mathrm{H}}}=1.822 \times 10^{18} \frac{\sigma_{r m s} d v}{G} \mathrm{~cm}^{-2}
$$

where $\sigma_{r m s}$ is in Jy, Where $G$ is the gain $(\mathrm{Jy} / \mathrm{K})$ of the Parkes telescope at $1.4 \mathrm{GHz}$, and $d v$ is the $2.6 \mathrm{~km} / \mathrm{s}$ velocity resolution of the cube. We calculate our $5 \sigma$ detection sensitivity using Eq. 4.3 where we assume a linewidth of $20 \mathrm{~km} / \mathrm{s}$ and we multiply the equation by five.

We also calculate the total dynamical mass of the system using the linewidth at $20 \%$ maximum, $W_{20}$. To do this, we first need an $\mathrm{H}$ I diameter, $D_{\mathrm{H} \text { I }}$, which we derive using the Broeils \& Rhee (1997) scaling relation:

$$
\log _{10}\left(\mathrm{D}_{\mathrm{HI}}\right)=0.99 \log _{10}\left(\mathrm{D}_{25}\right)+0.24
$$

Here, the optical diameter measured at the $25^{\text {th }}$ mag $\operatorname{arcsec}^{-2}$ isophote is taken from 
LEDA. This angular diameter can then be converted to a physical H I radius, $r_{\mathrm{HI}}$, to derive the dynamical mass:

$$
M_{\mathrm{dyn}}=2.3 \times 10^{5}\left(\frac{v_{\text {rot }} / \sin (i)}{\mathrm{km} \mathrm{s}^{-1}}\right)^{2}\left(\frac{r_{\mathrm{HI}}}{\mathrm{kpc}}\right) \mathrm{M}_{\odot}
$$

where $v_{r o t}=W_{20} / 2$. Each of these derived galaxy parameters can be found in Table 4.3. So far, we have defined several types of masses. The H I mass $M_{\mathrm{HI}}$, refers to the mass in the disk of the galaxy, derived from the galaxy's integrated spectral profile. The dynamical mass $M_{\mathrm{dyn}}$, is a mass derived from the kinematics of the gas and tells us the amount of mass we would expect based on the rotation of the galaxy. The virial mass $\left(M_{v i r}\right)$ and the dark matter halo mass $\left(M_{\text {halo }}\right)$ are equivalent, and refer to the total mass of the dark matter halo. In this work, we use both terms dependent on the context or the literature the values are taken from.

\subsubsection{Differences in Analysis}

A similar analysis procedure to that done in Chapter 3 was performed on this data set with a few changes. Each IMAGINE data cube was regridded to a slightly larger pixel size of $8^{\prime \prime} \times 8^{\prime \prime}$ due to the size of the resulting data cubes. Since each cube extends past the virial radius of each galaxy, we extracted a subcube that encloses the virial radius, but excludes the edges of the cube and subsequently the regions where the noise is non-uniform. On these new subcubes, we performed the following analysis.

We created moment 0 maps for the entire RA/Dec region of the subcubes, 


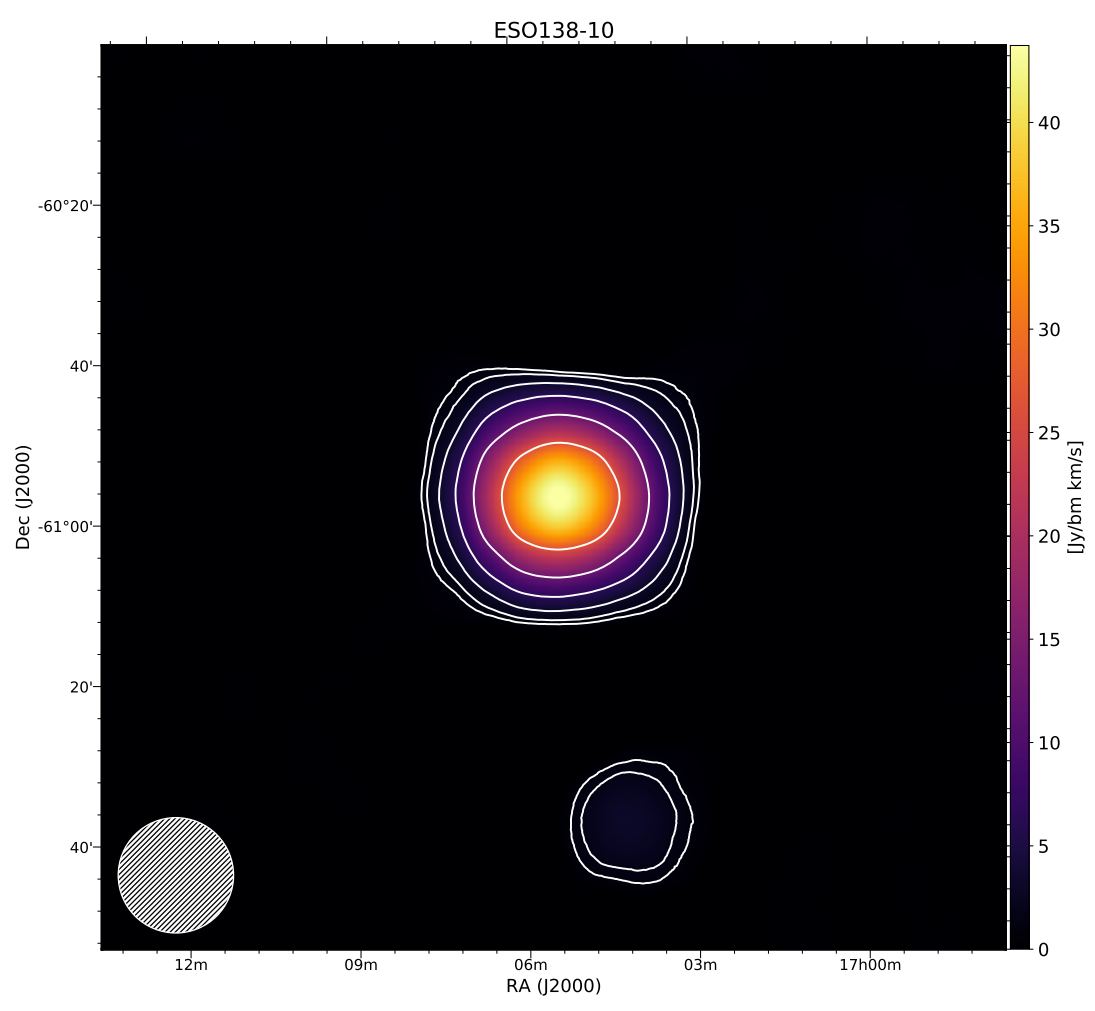

Figure 4.2: Integrated intensity map (Moment 0 ). Contours at column density levels of $N_{\mathrm{HI}}=3,5,10,20,40$, and $80 \times 10^{18} \mathrm{~cm}^{-2}$. The Parkes $14.4^{\prime} \mathrm{FWHM}$ beamsize indicated in lower left corner. 

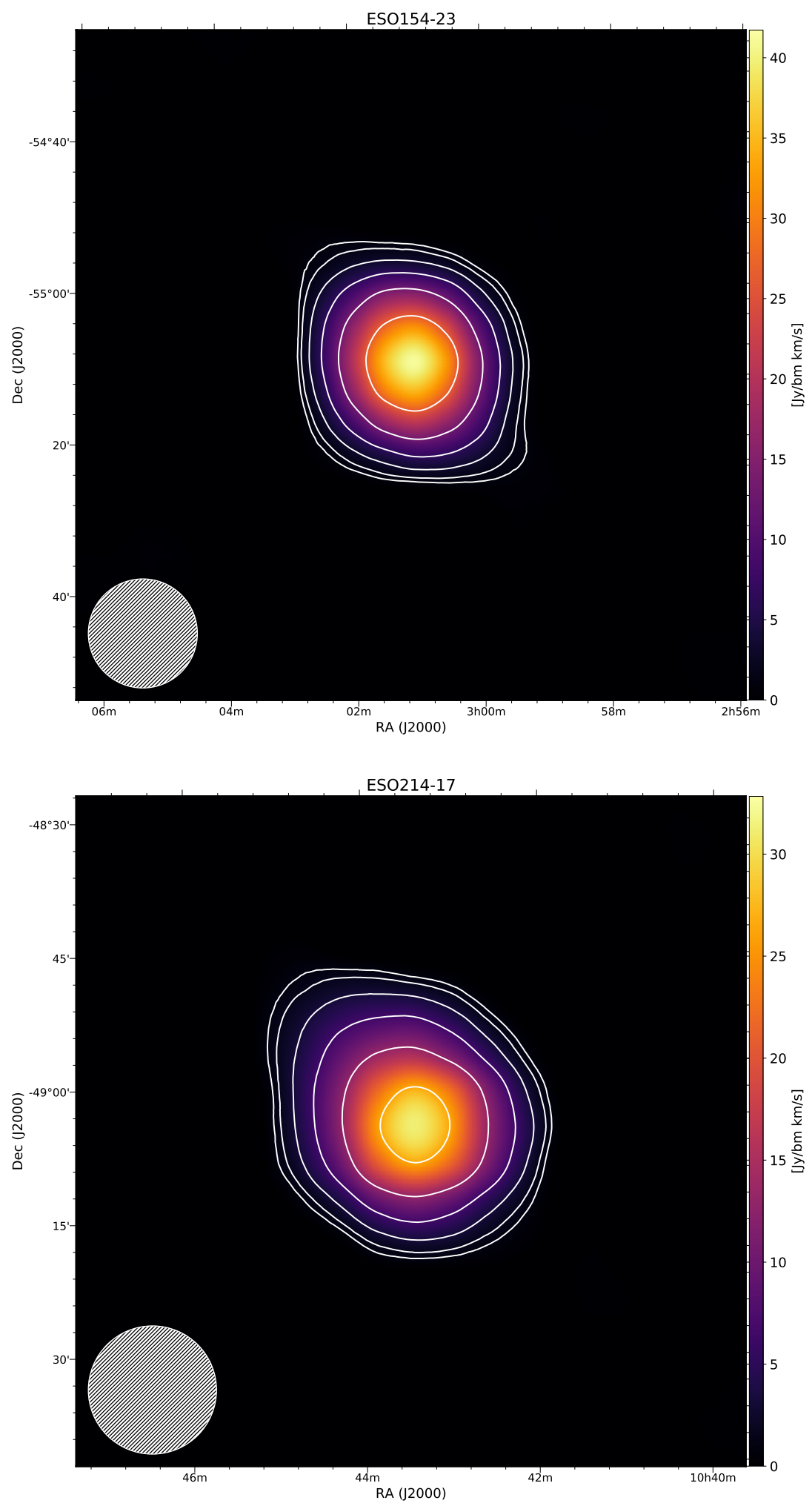

Figure 4.3: Same as Figure 4.2, but for ESO154-23 (top) and ESO214-17 (bottom). 
IC5201
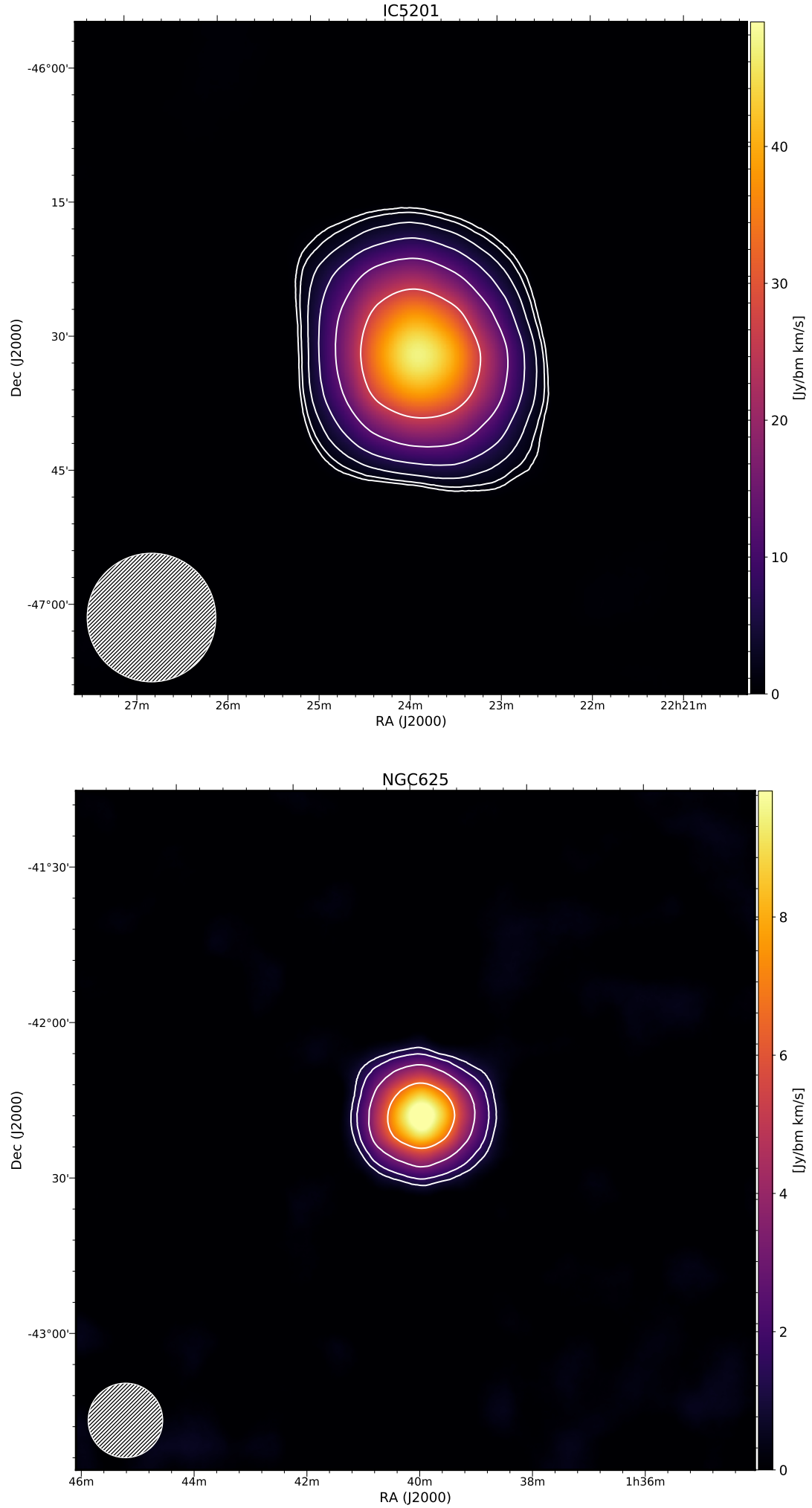

Figure 4.4: Same as Figure 4.2, but for IC5201 (top) and NGC625 (bottom). 

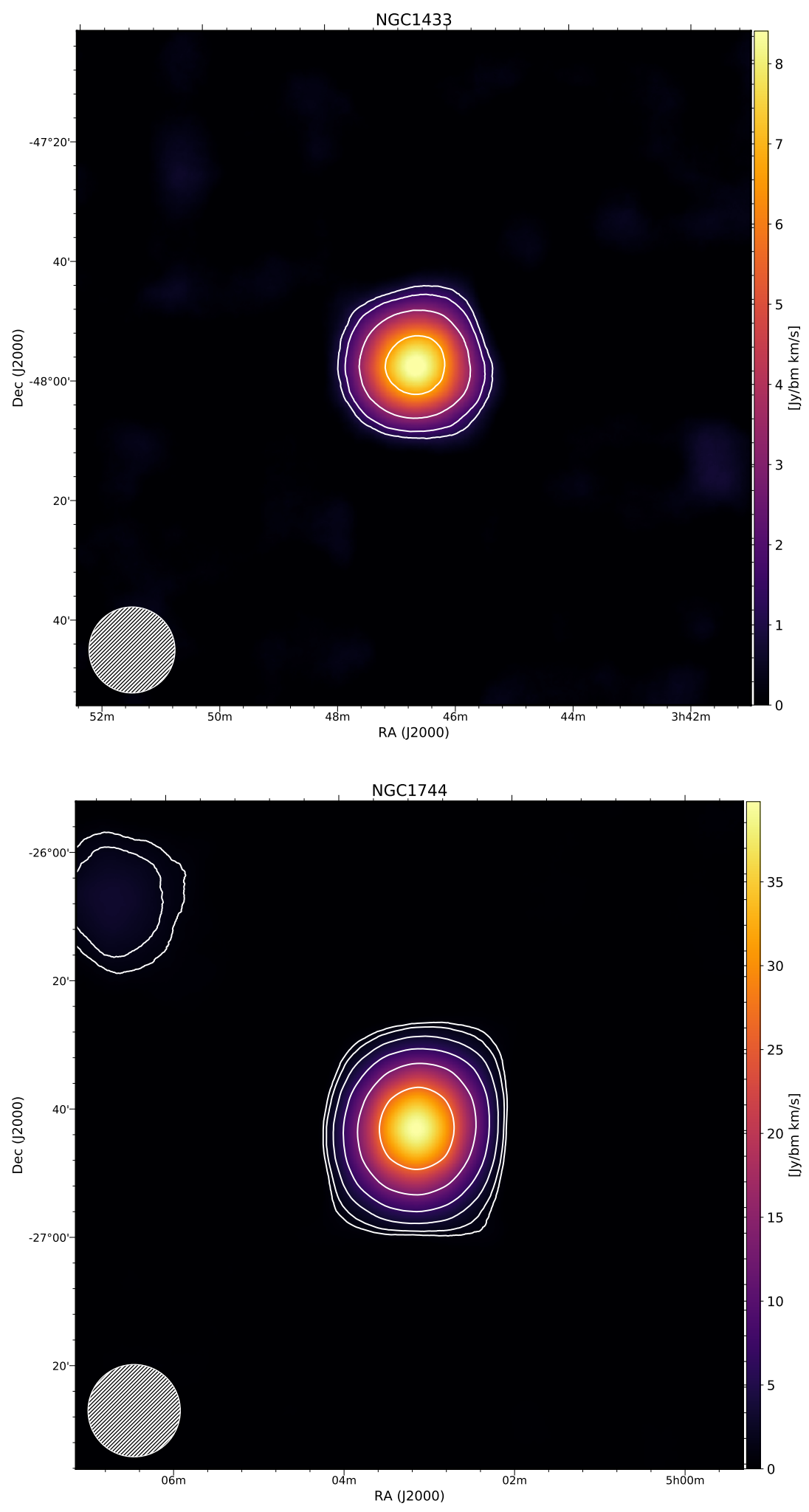

Figure 4.5: Same as Figure 4.2, but for NGC1433 (top) and NGC1744 (bottom). 

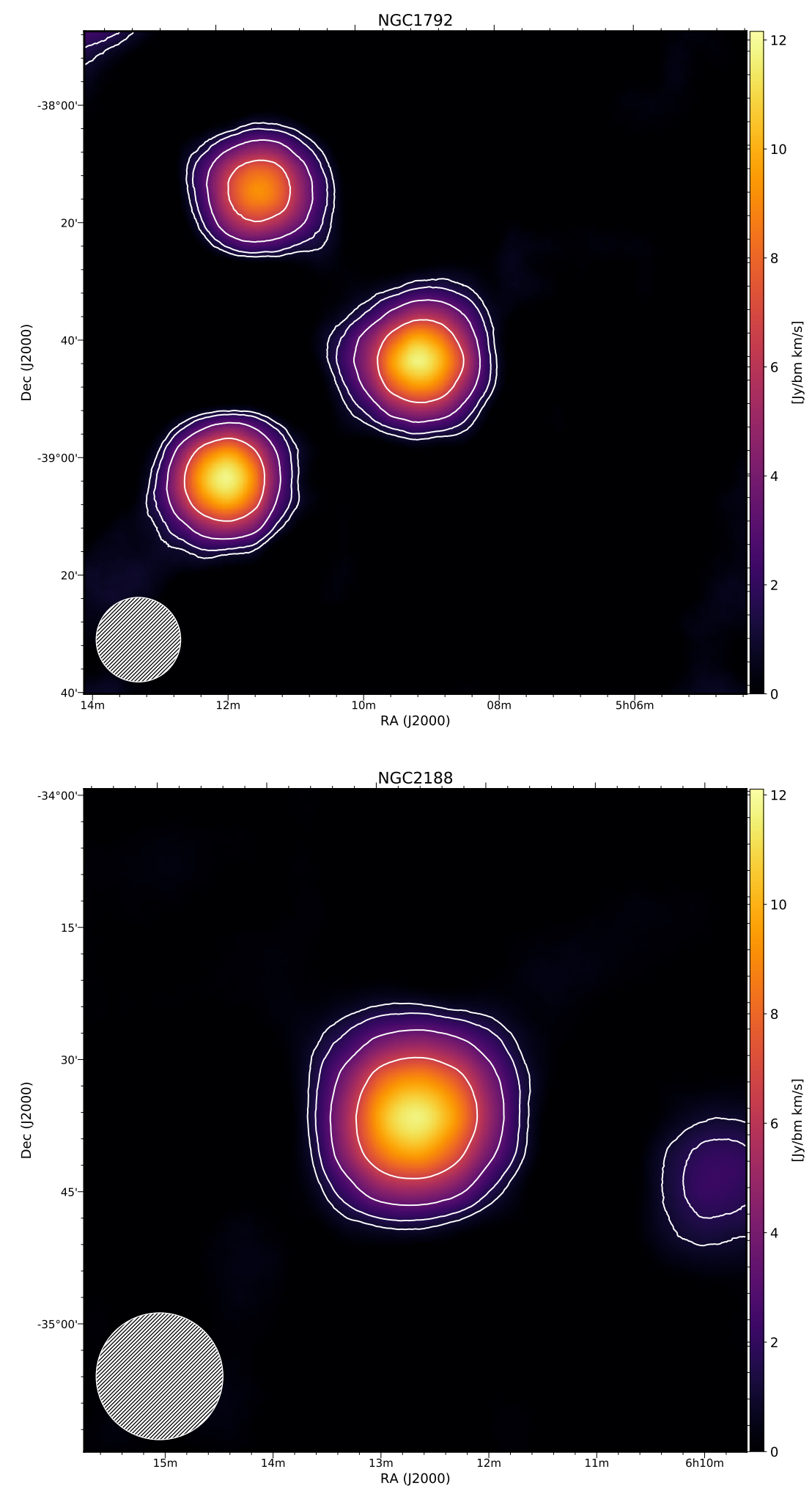

Figure 4.6: Same as Figure 4.2, but for NGC1792 (top) and NGC2188 (bottom). 

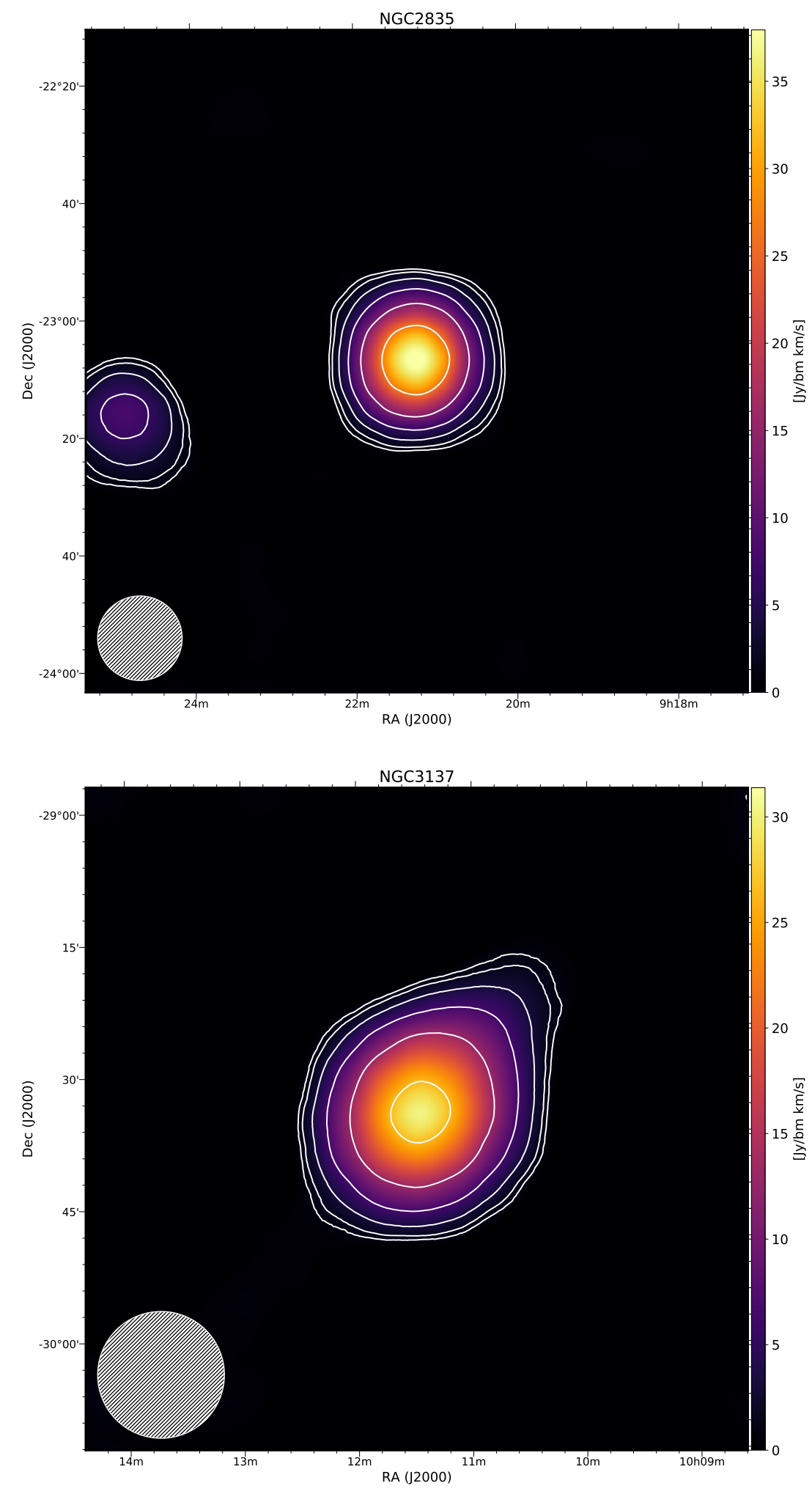

Figure 4.7: Same as Figure 4.2, but for NGC2835 (top) and NGC3137 (bottom). 

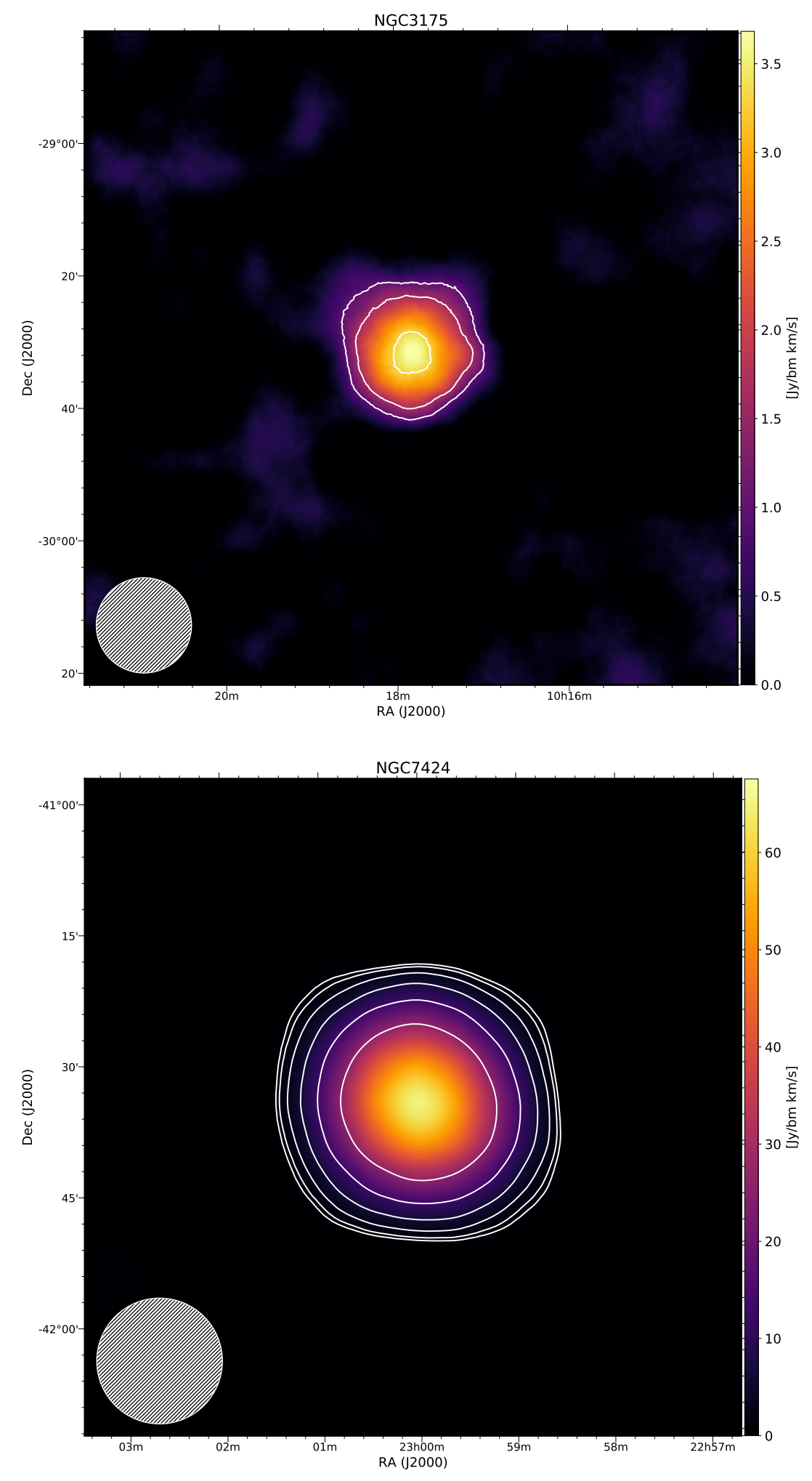

Figure 4.8: Same as Figure 4.2, but for NGC3175 (top) and NGC7424 (bottom). 


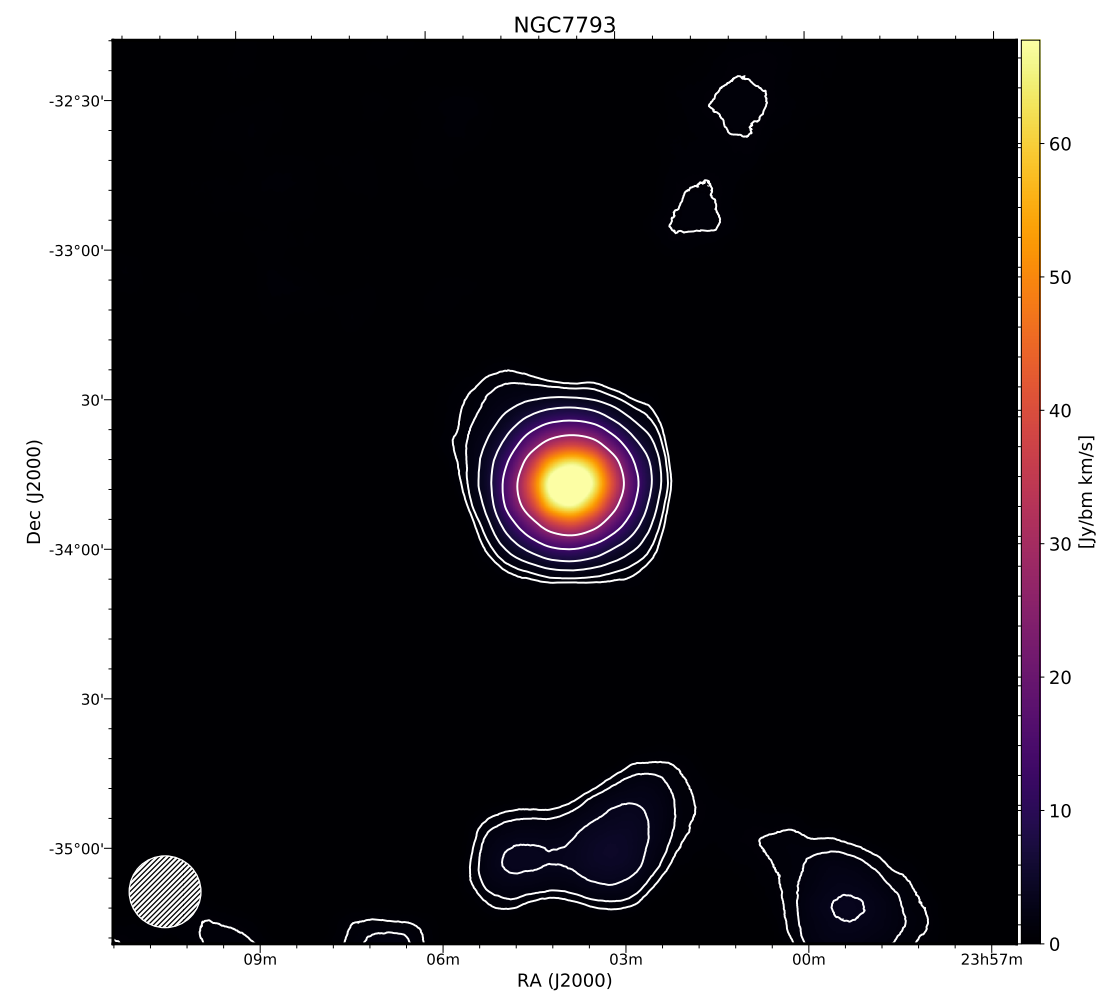

Figure 4.9: Same as Figure 4.2, but for NGC7793.

summing along the velocity axis in the range specific to that galaxy's profile. These can be seen in Figures 4.2 through 4.11, where contours are set at constant column density levels of $N_{\mathrm{HI}}=3,5,10,20,40$, and $80 \times 10^{18} \mathrm{~cm}^{-2}$ for Figures 4.2 through 4.9 and at $N_{\mathrm{HI}}=8,10,20,40$, and $80 \times 10^{18} \mathrm{~cm}^{-2}$ for maps with higher noise for Figures 4.10 through 4.11. As we described in Chapter 3, we wanted to calculate a signal-to-noise map for each cube, however for this data set, we chose to Hanning smooth the data in order to reduce Gibbs ringing from Galactic emission. As a result, we calculated our noise maps in another way. We used the following calculation from Verheijen \& Sancisi (2001) who showed that when observations are made using a 

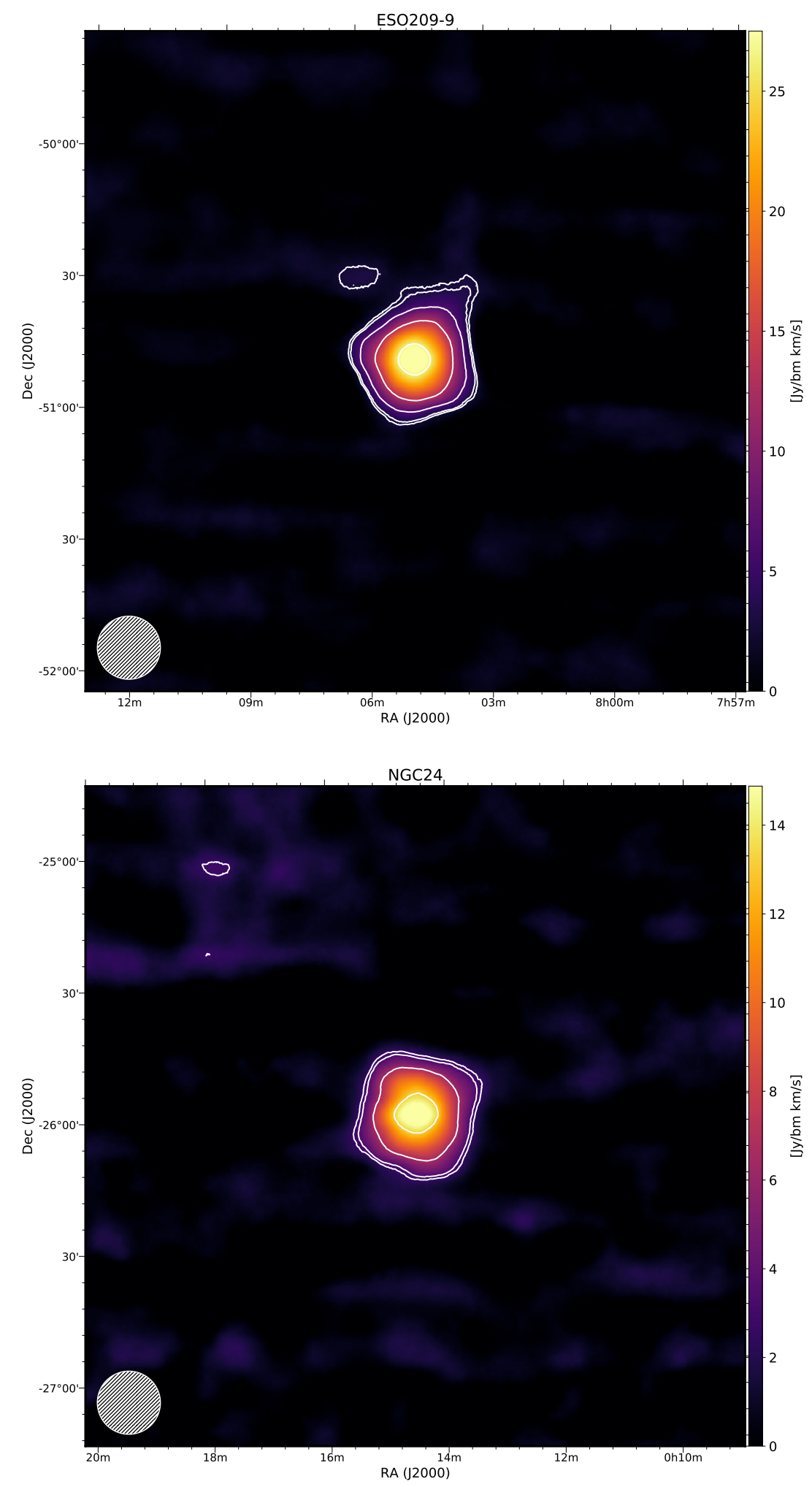

Figure 4.10: Integrated intensity map (Moment 0) for ESO209-9 (top) and NGC24 (bottom). Contours at column density levels of $N_{\mathrm{HI}}=8,10,20,40$, and $80 \times 10^{18} \mathrm{~cm}^{-2}$. The Parkes 14.4' FWHM beamsize indicated in lower left corner. 

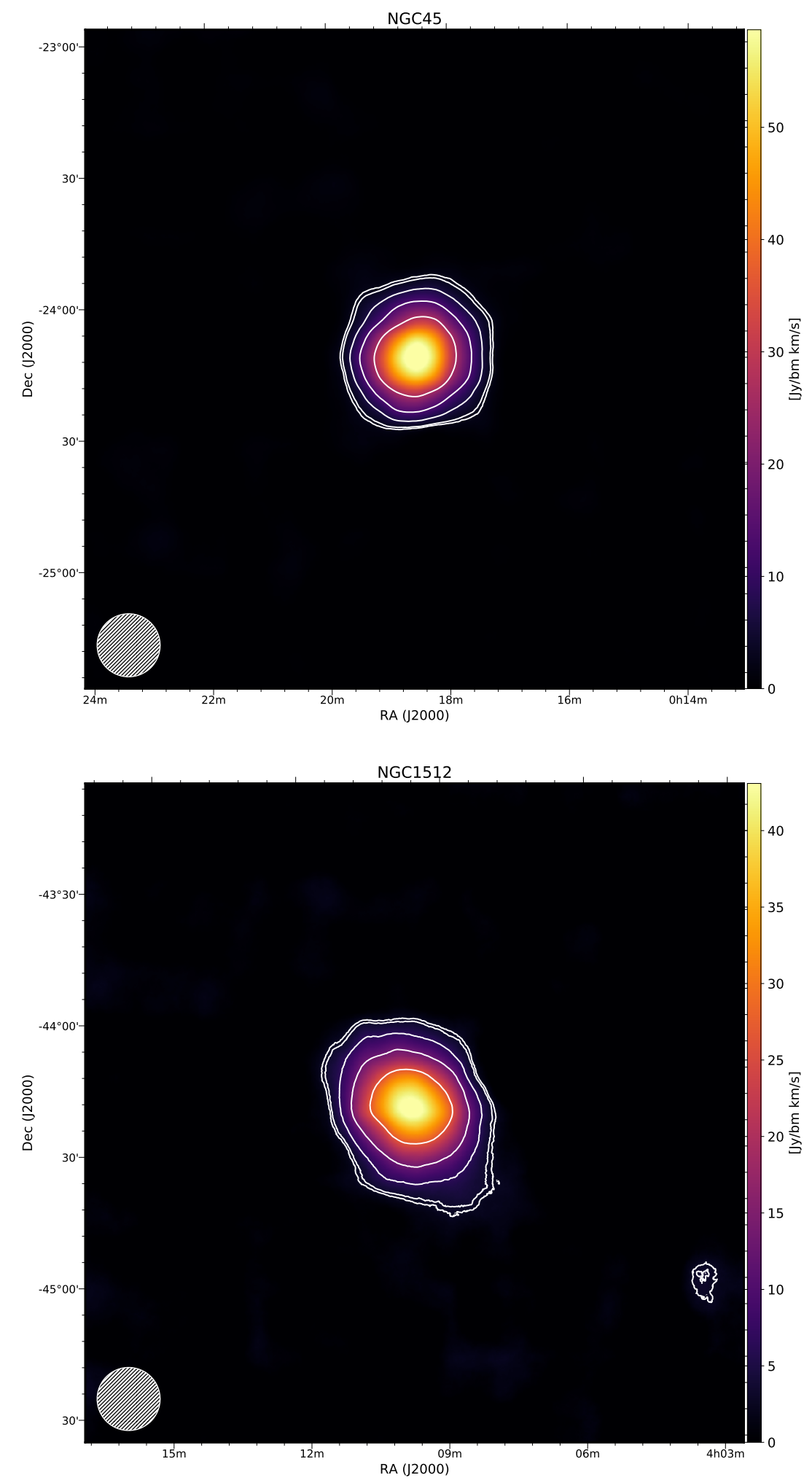

Figure 4.11: Same as Figure 4.10, but for NGC45 (top) and NGC1512 (bottom). 
Hanning taper, the noise at each pixel in the integrated map increases by:

$$
\sigma_{N}=\sqrt{N-\frac{3}{4}} \frac{4}{\sqrt{6}} \sigma_{r m s}
$$

rather than by a factor of $\sqrt{N}$.

We calculate the cumulative H I mass in each cube and plot this as a function of column density. Doing this allows us to quantify the amount of H I gas in the cube that falls below column density levels of $N_{\mathrm{HI}}=10^{19} \mathrm{~cm}^{-2}$, which we define as diffuse H I. Additionally, each galaxy's cumulative H I mass is compared to a Gaussian model of the Parkes beam. This is one way in which we test whether our sources are resolved within the Parkes beam, where H I that lies above this model is tentatively considered resolved in the Parkes 14.4' FWHM beam width. We are satisfied with the use of a Gaussian beam model, in aggreement with Staveley-Smith et al. (1996), after a comparison of the results from Chapter 3 with a Gaussian beam model, resulting in $\mathrm{a} \sim 1 \%$ difference in the amount of diffuse $\mathrm{H}$ I gas below column density levels of $N_{\mathrm{HI}}=10^{19} \mathrm{~cm}^{-2}$.

The extent of the radial $N_{\mathrm{HI}}$ maps is much greater for our IMAGINE galaxies than for our MHONGOOSE data set. Because we mapped out to or beyond the virial radius of each galaxy, the azimuthally averaged $\mathrm{H}$ I column density levels give us a complete picture of the H I throughout the virial volume of each galaxy. With $8^{\prime \prime}$ pixels in the cubes, and a 14.4' FWHM Parkes beam size, we set the radius of the innermost annulus to be half the size of the Parkes beam, or 54 pixels. Each subsequent annulus is at a multiple of 54 pixels out to the largest multiple within 
the subcube. Since the angular extent of the virial radius changes per galaxy, the number of annuli varies per galaxy. Within each annulus, the $N_{\mathrm{HI}}$ in our masked image is averaged and plotted as a function of physical radius in Figures 4.12 through 4.29. Similar to Chapter 3, we chose to use the masked image in order to reduce the chances of quantifying noise as low column density signal. The Gaussian beam model is also plotted here, allowing us to compare the radius of the Parkes data to the radius of a Gaussian beam model at a constant column density. In many cases, at a given $N_{\mathrm{HI}}$ level, the smallest radii of the Parkes data fall on or below that of the model, indicating that these galaxies are either barely resolved or unresolved in the Parkes beam. However, for our purposes, this will not effect our analysis as we are mostly interested in the regions outside the disk of the galaxy, which are clearly well-resolved.

Using our unmasked images, we track the cumulative flux as we extend out to the virial radius of each galaxy. This is shown in Figures 4.12 through 4.29. These plots quantify the total amount of $\mathrm{H}$ I flux within the dark matter halo by summing the flux in each annulus out to or beyond the virial radius of each galaxy.

\subsection{Results}

\subsubsection{Results from radial and cumulative analysis}

The results from the analysis described in the previous section is presented here for each of our IMAGINE galaxies. Each galaxy is represented in Figures 4.12 through 4.29, where we show the integrated H I spectrum, the cumulative H I mass ratio as a 

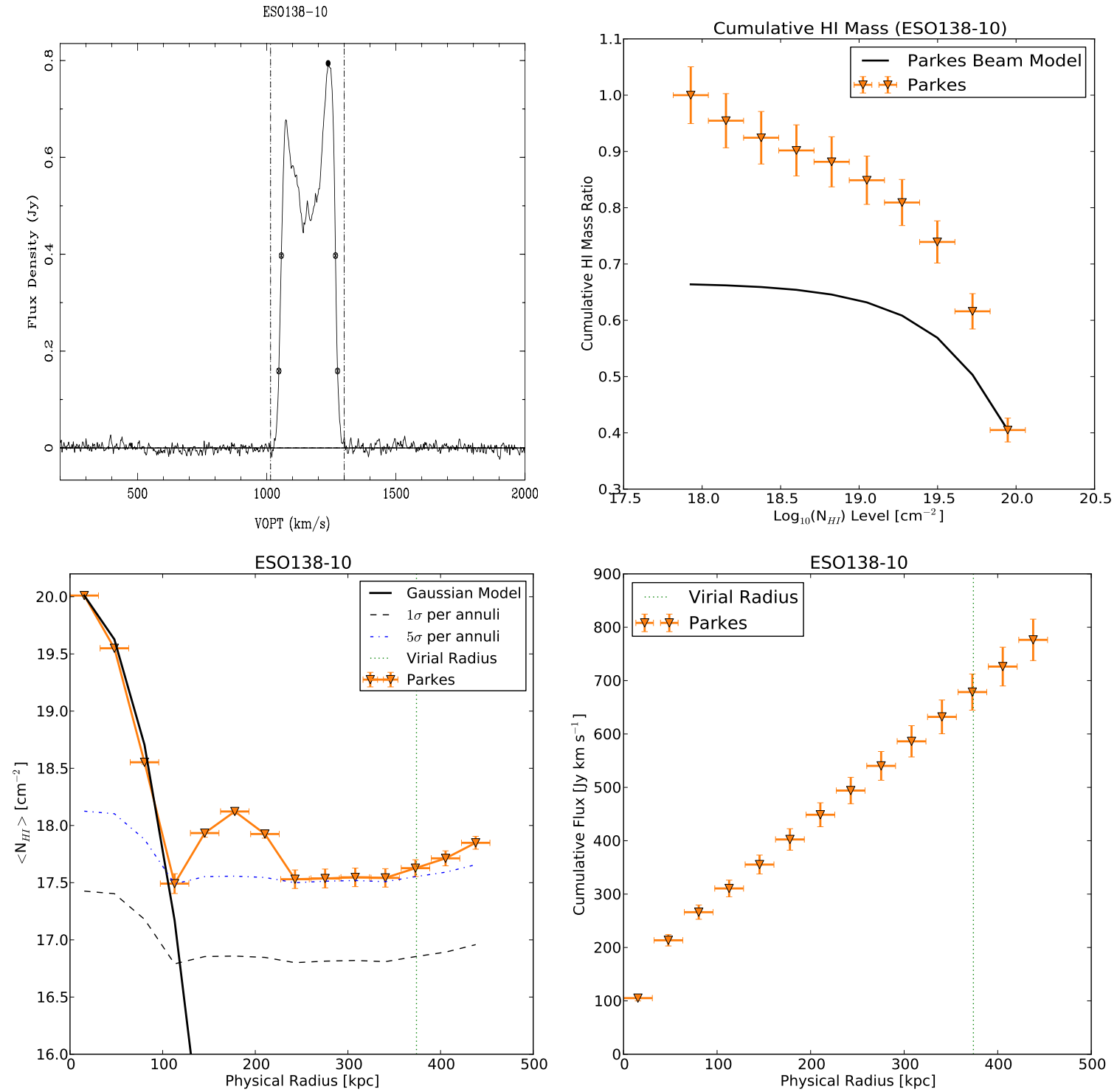

Figure 4.12: Top left: Total integrated H I profile - measured between the dashed and dotted vertical lines. Open circles and X's indicate 20\% and 50\% maximum velocity widths. Filled circles represent the mean velocity. Top right: Cumulative H I mass - total H I mass within the virial volume is plotted by the fraction of gas in column density bins, and compared to the Gaussian representation of the Parkes beam, scaled to the peak column density. Lower left: Azimuthally averaged $N_{\mathrm{HI}}$ - column density averaged over annuli extending from the center of the galaxy to beyond the virial radius, and compared to the Gaussian beam model. Black dashed line characterizes the $1 \sigma$ noise in each annulus, and the blue dot-dash line represents the $5 \sigma$ noise in each annulus. Lower right: Cumulative flux - Flux in each of those annuli are summed to obtain a measure of the total flux out beyond the virial radius. 

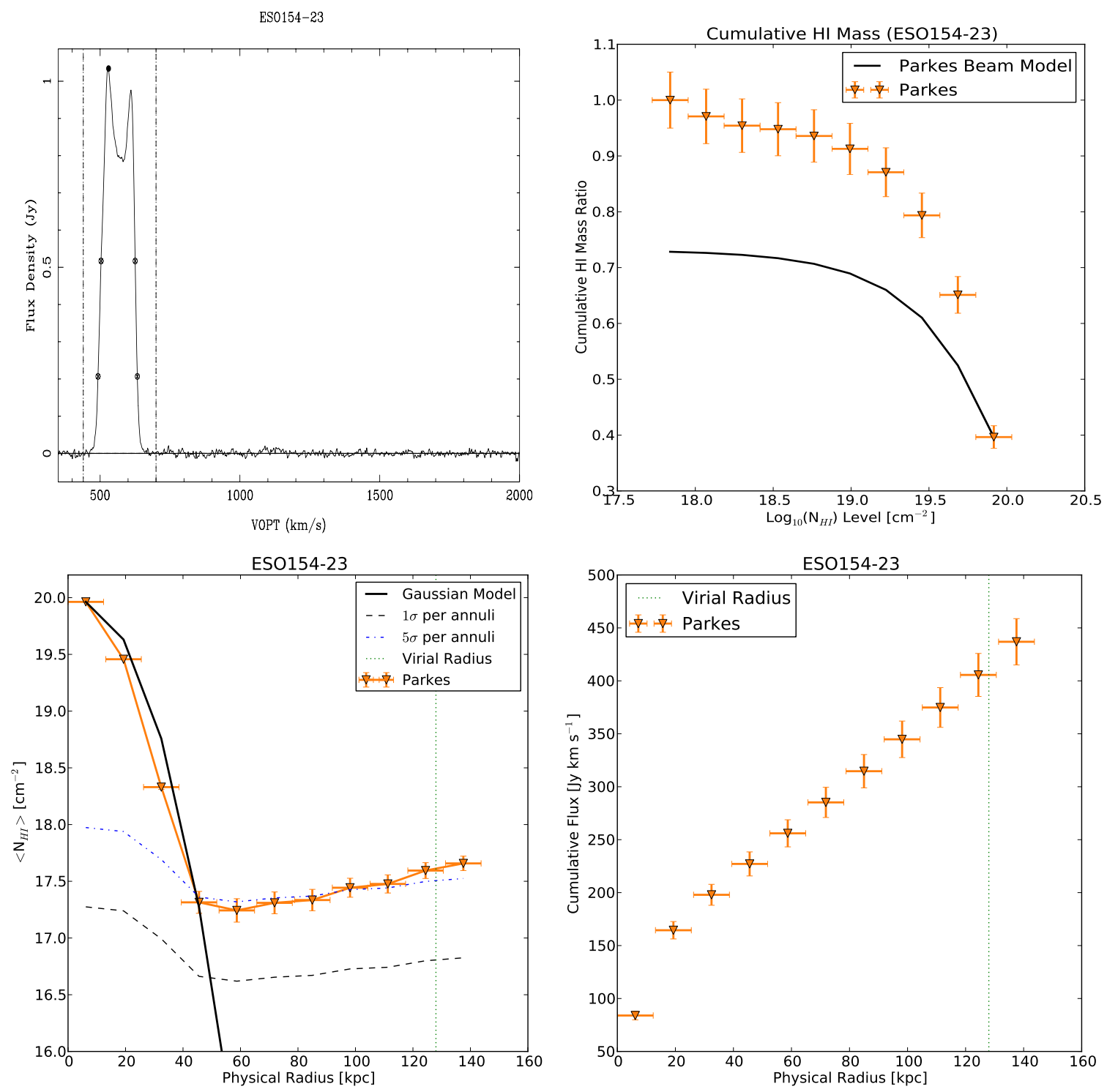

Figure 4.13: Same as Figure 4.12, but for ESO154-23. 

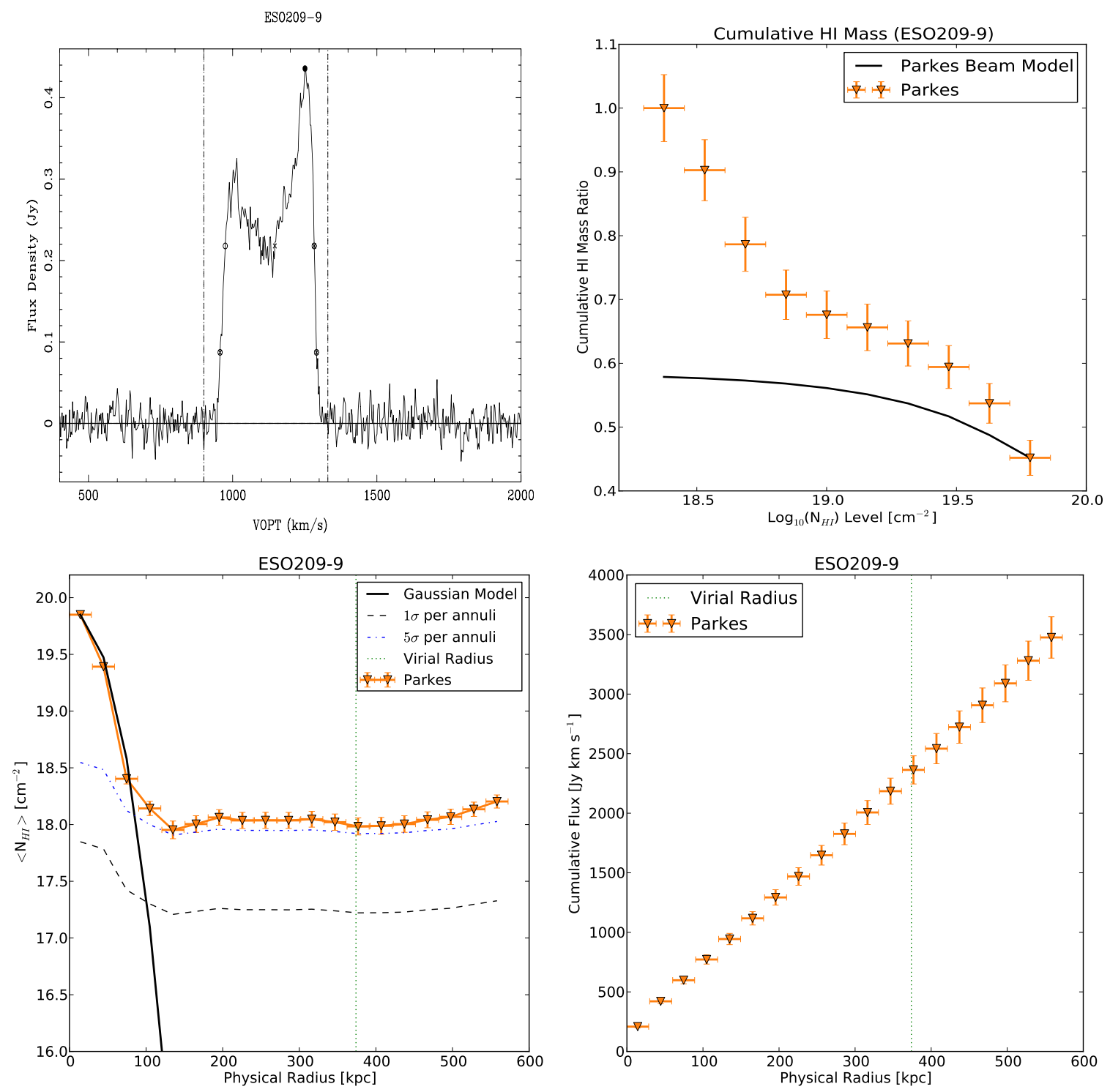

Figure 4.14: Same as Figure 4.12, but for ESO209-9. 

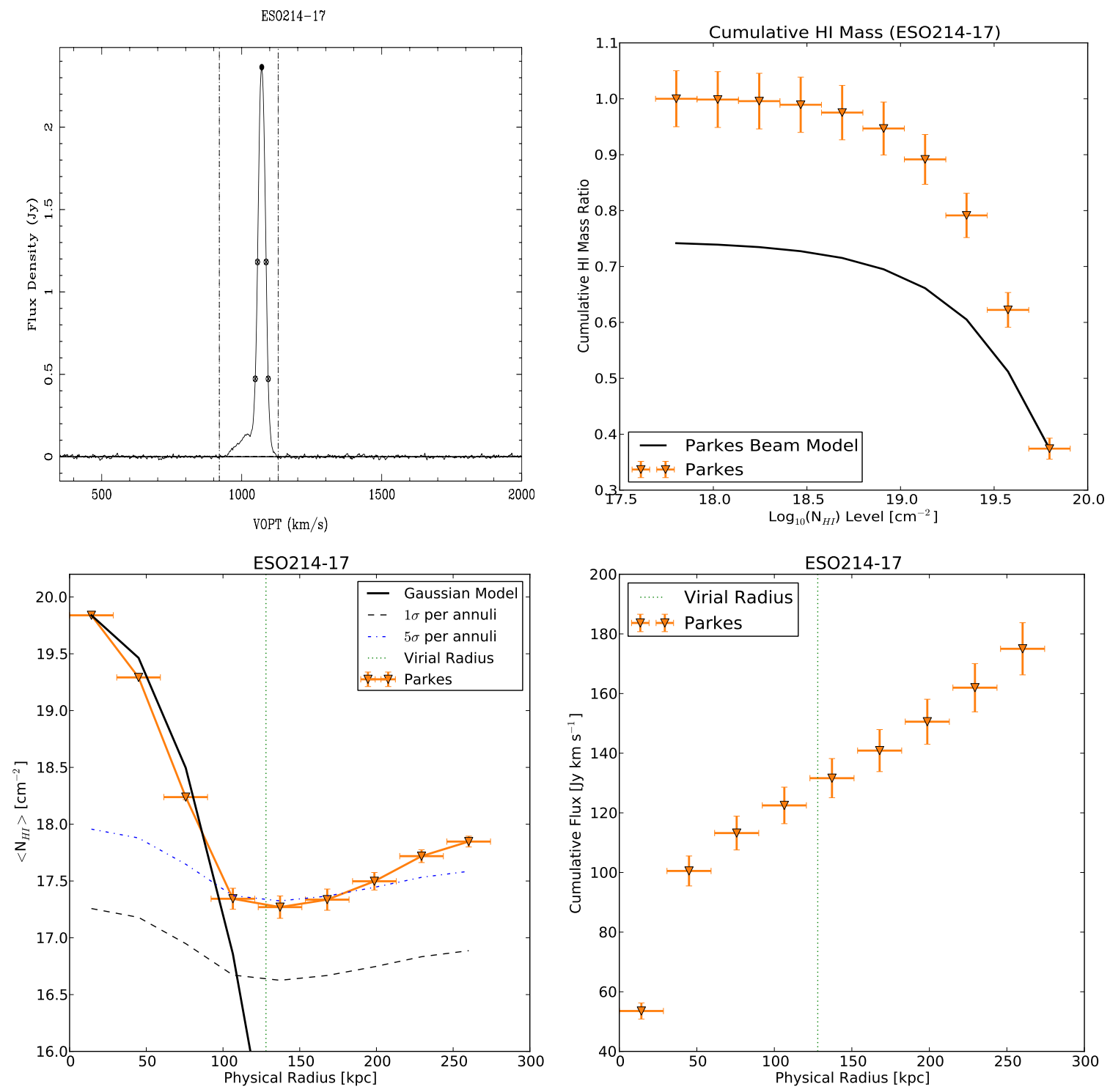

Figure 4.15: Same as Figure 4.12, but for ESO138-10. 

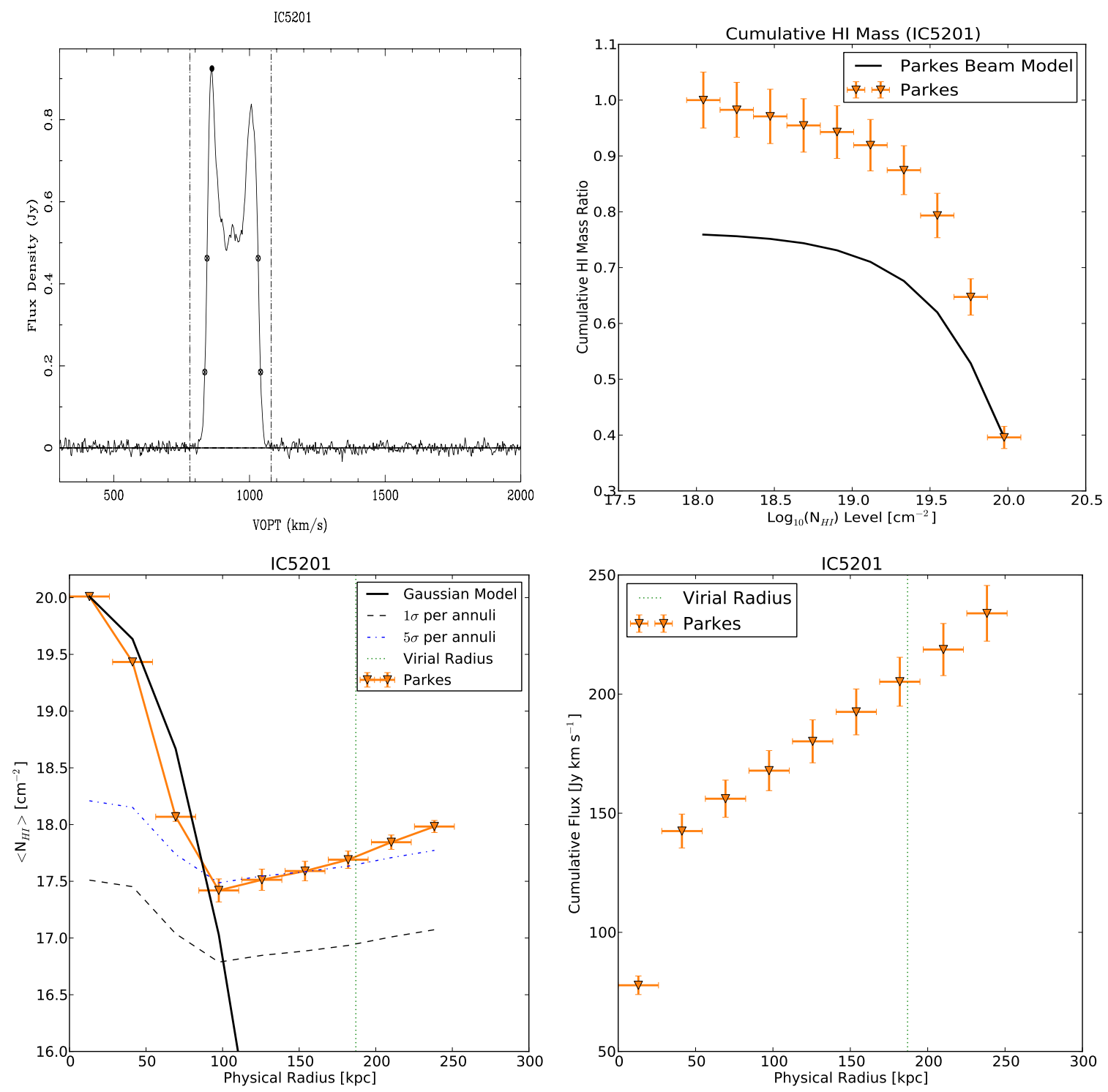

Figure 4.16: Same as Figure 4.12, but for IC5201. 

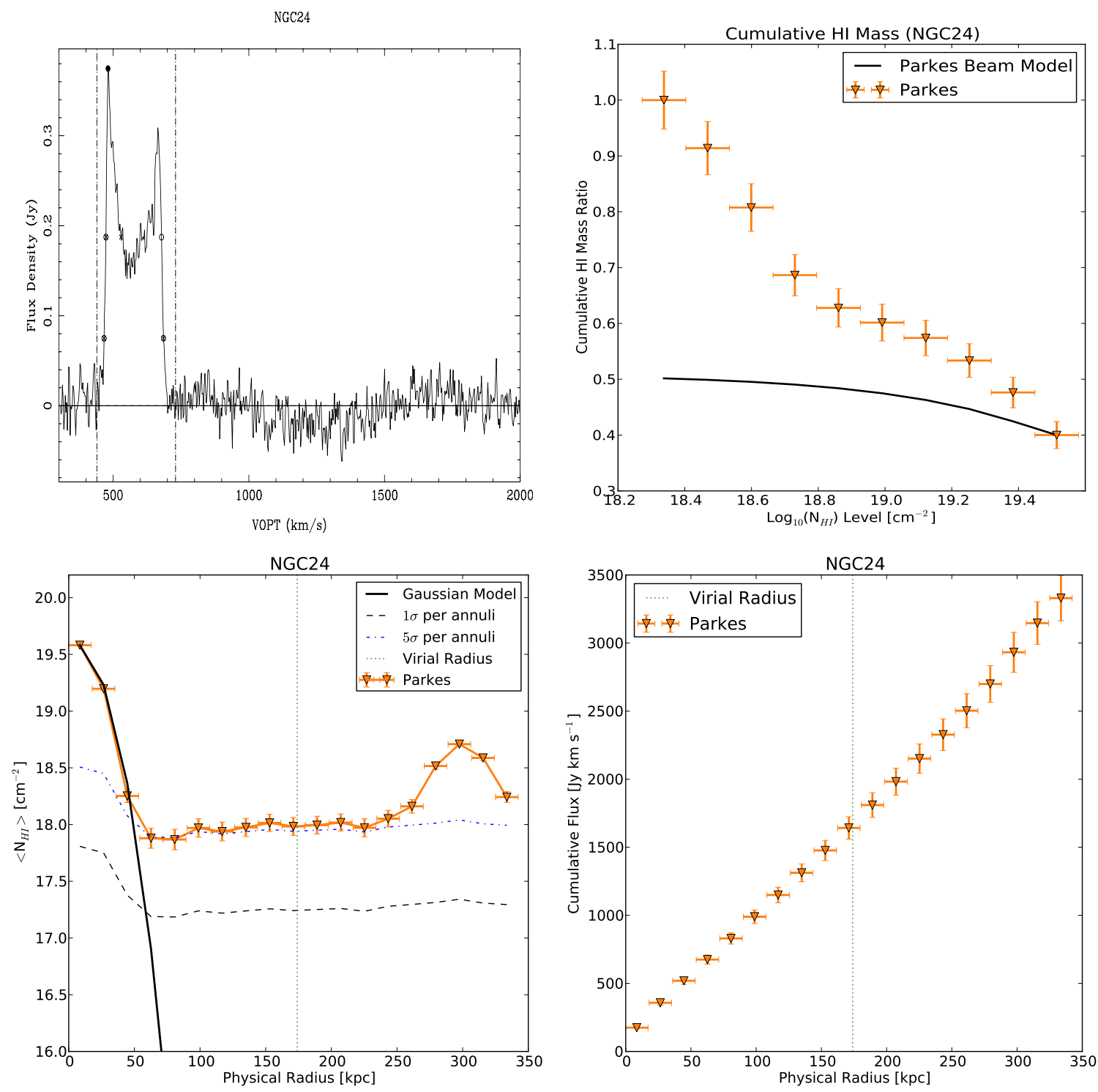

Figure 4.17: Same as Figure 4.12, but for NGC24. 

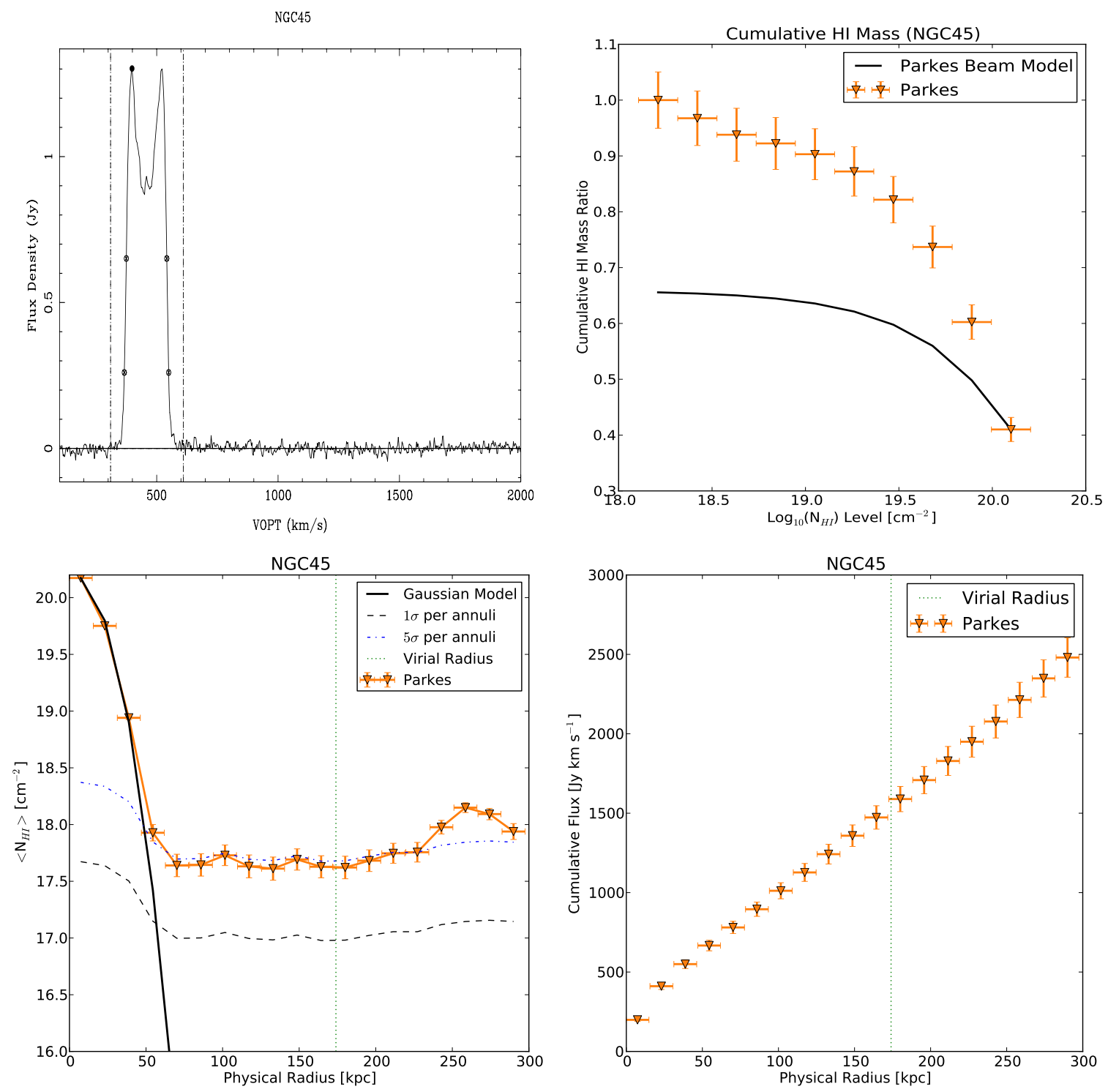

Figure 4.18: Same as Figure 4.12, but for NGC45. 

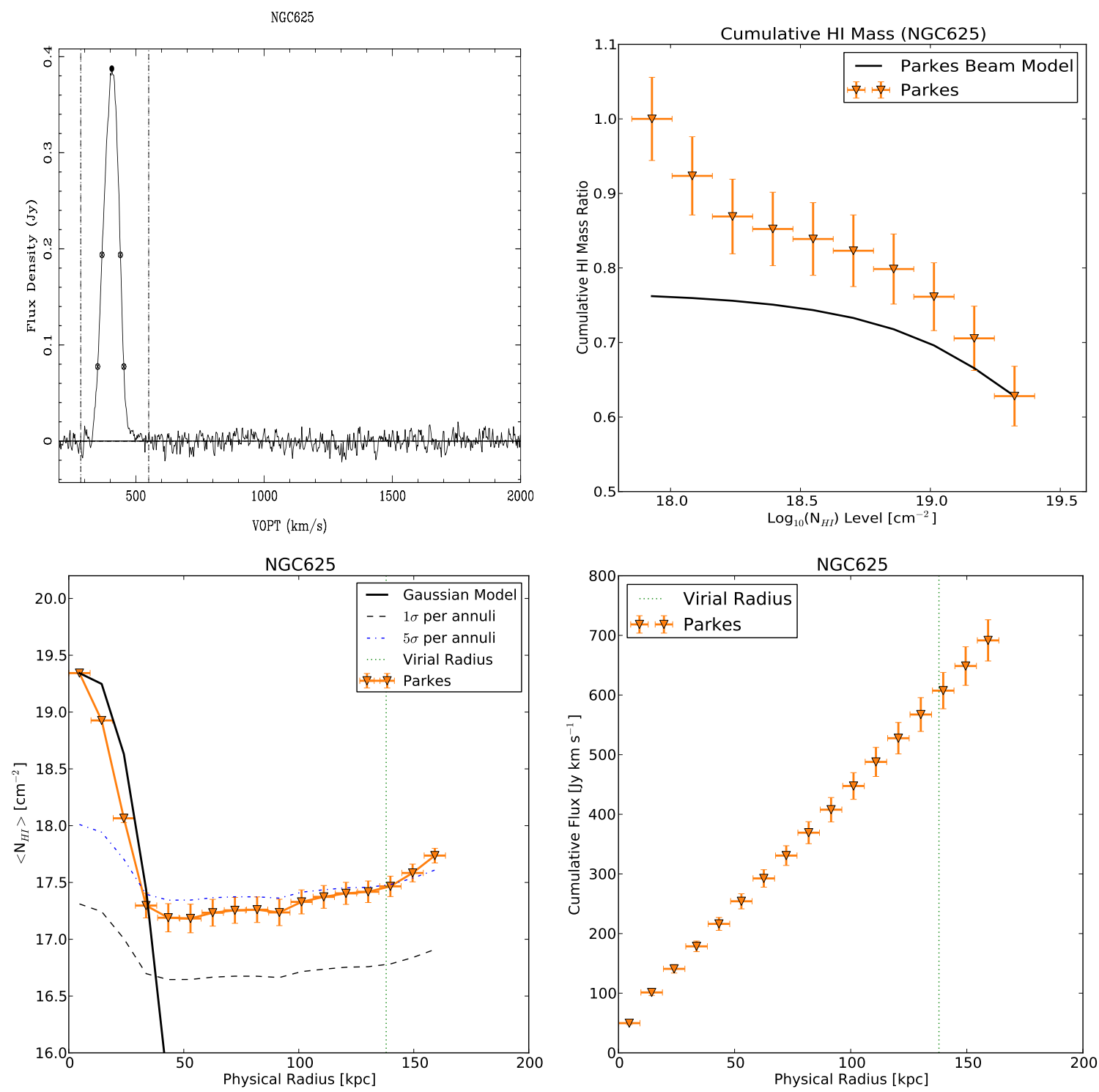

Figure 4.19: Same as Figure 4.12, but for NGC625. 

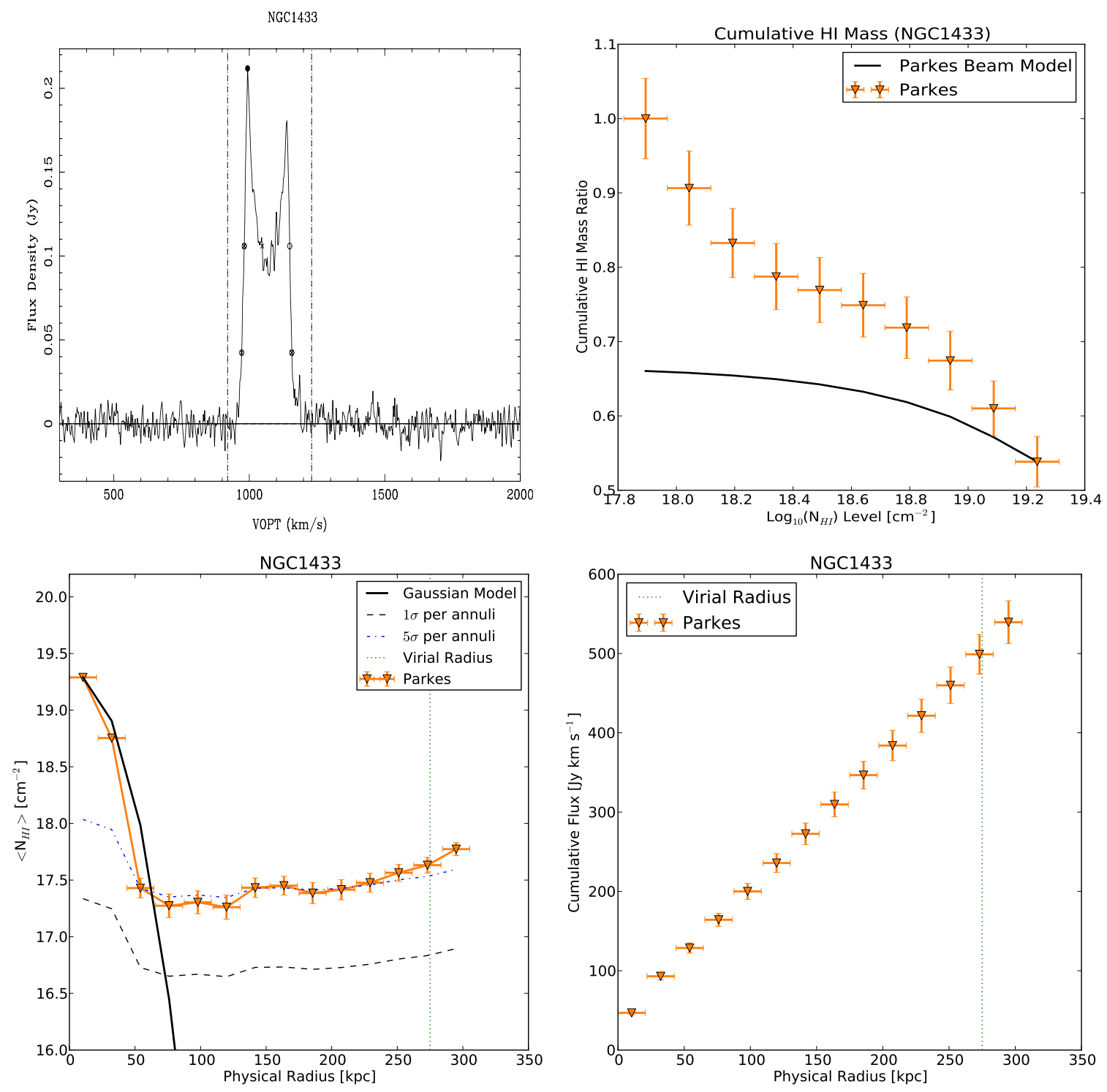

Figure 4.20: Same as Figure 4.12, but for NGC1433. 

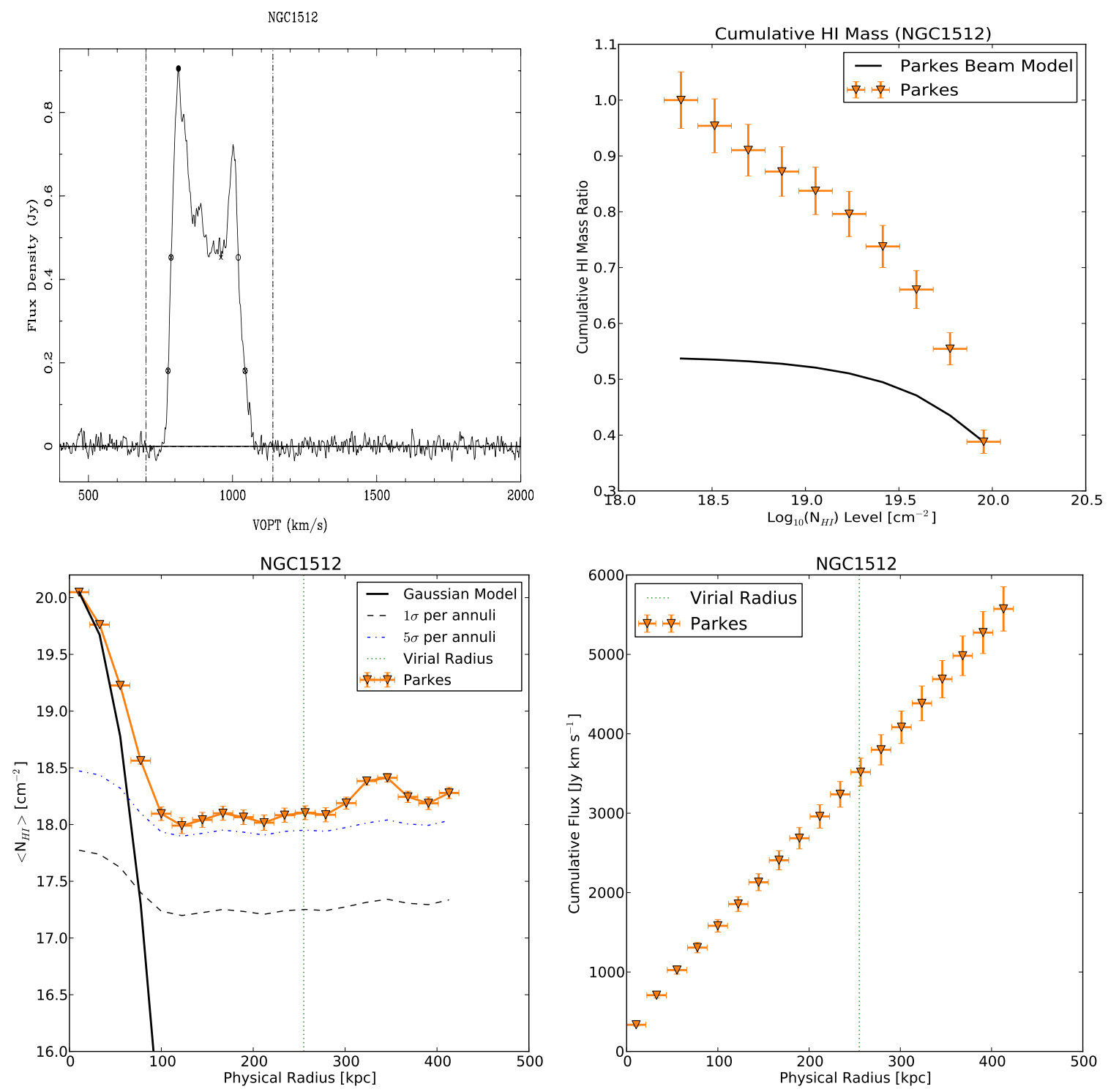

Figure 4.21: Same as Figure 4.12, but for NGC1512. 

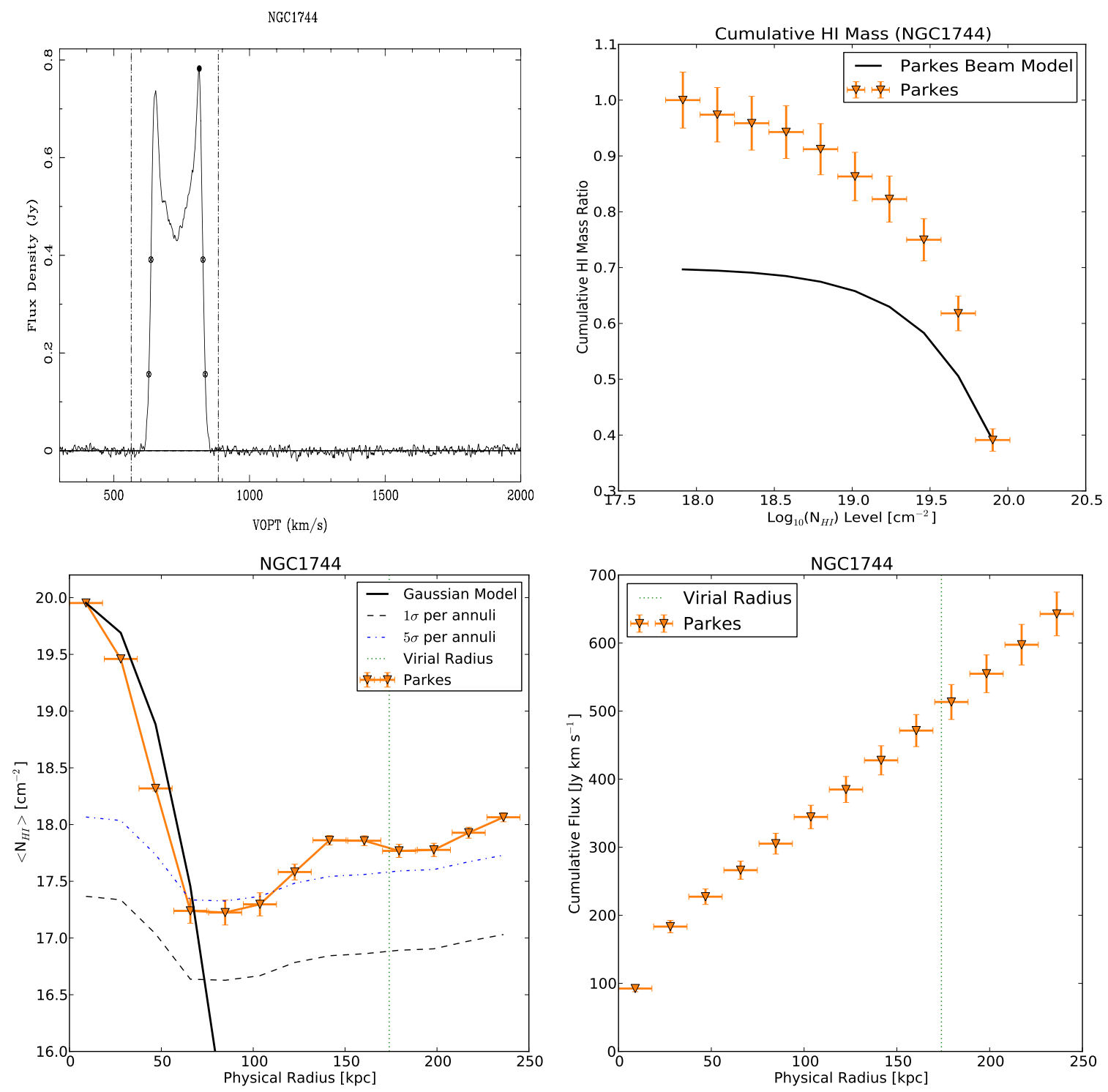

Figure 4.22: Same as Figure 4.12, but for NGC1744. 

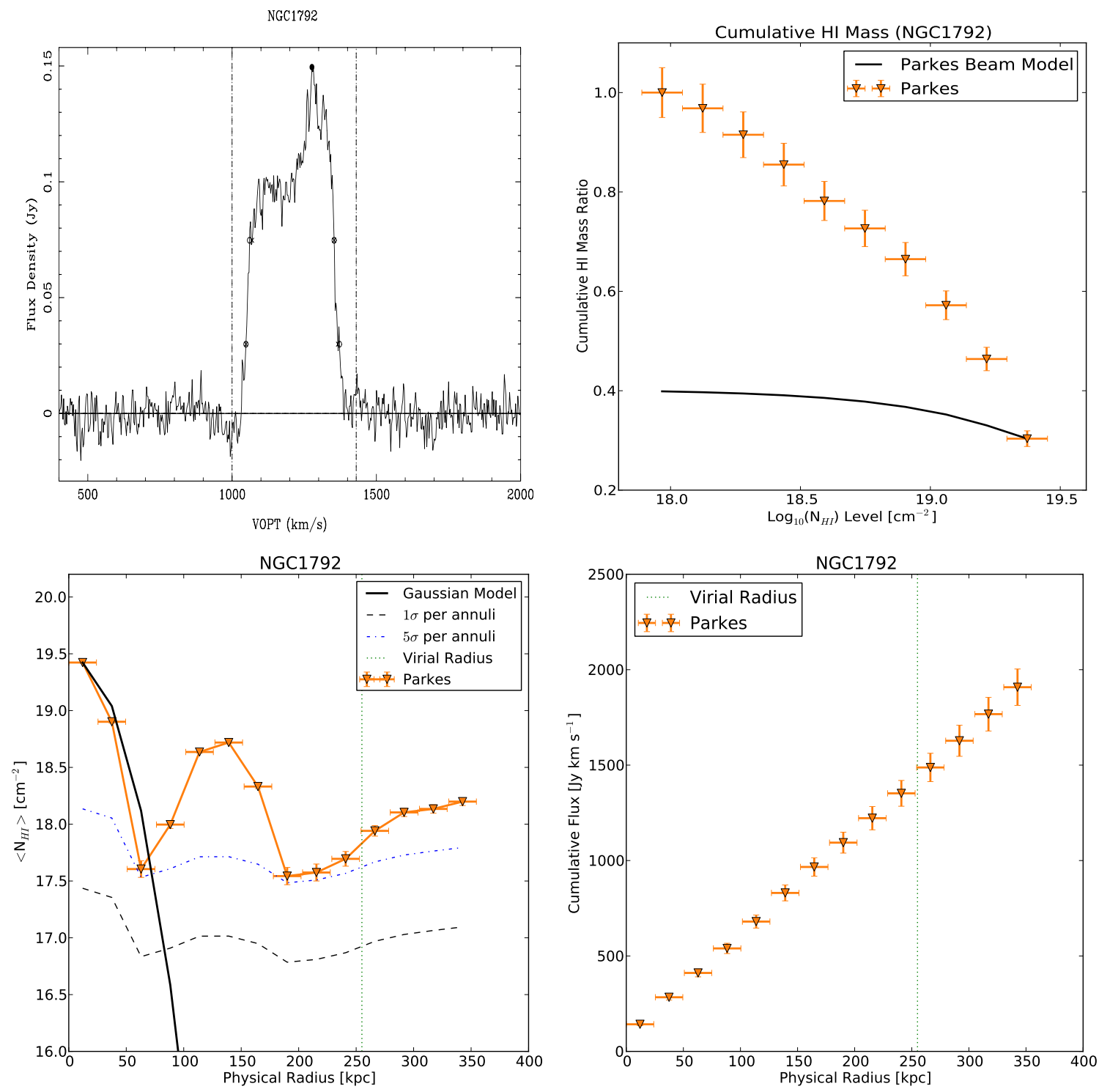

Figure 4.23: Same as Figure 4.12, but for NGC1792. 

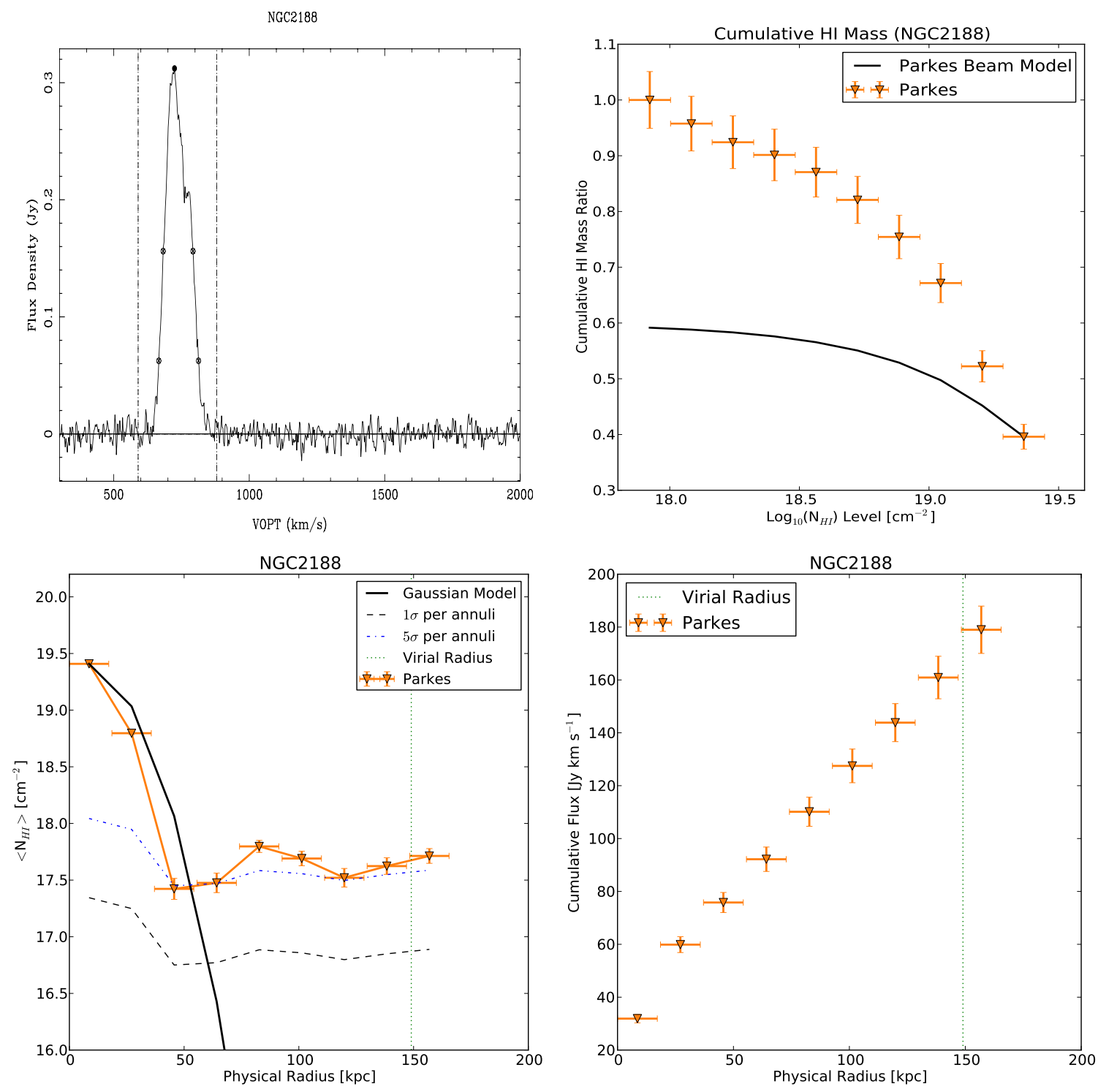

Figure 4.24: Same as Figure 4.12, but for NGC2188. 

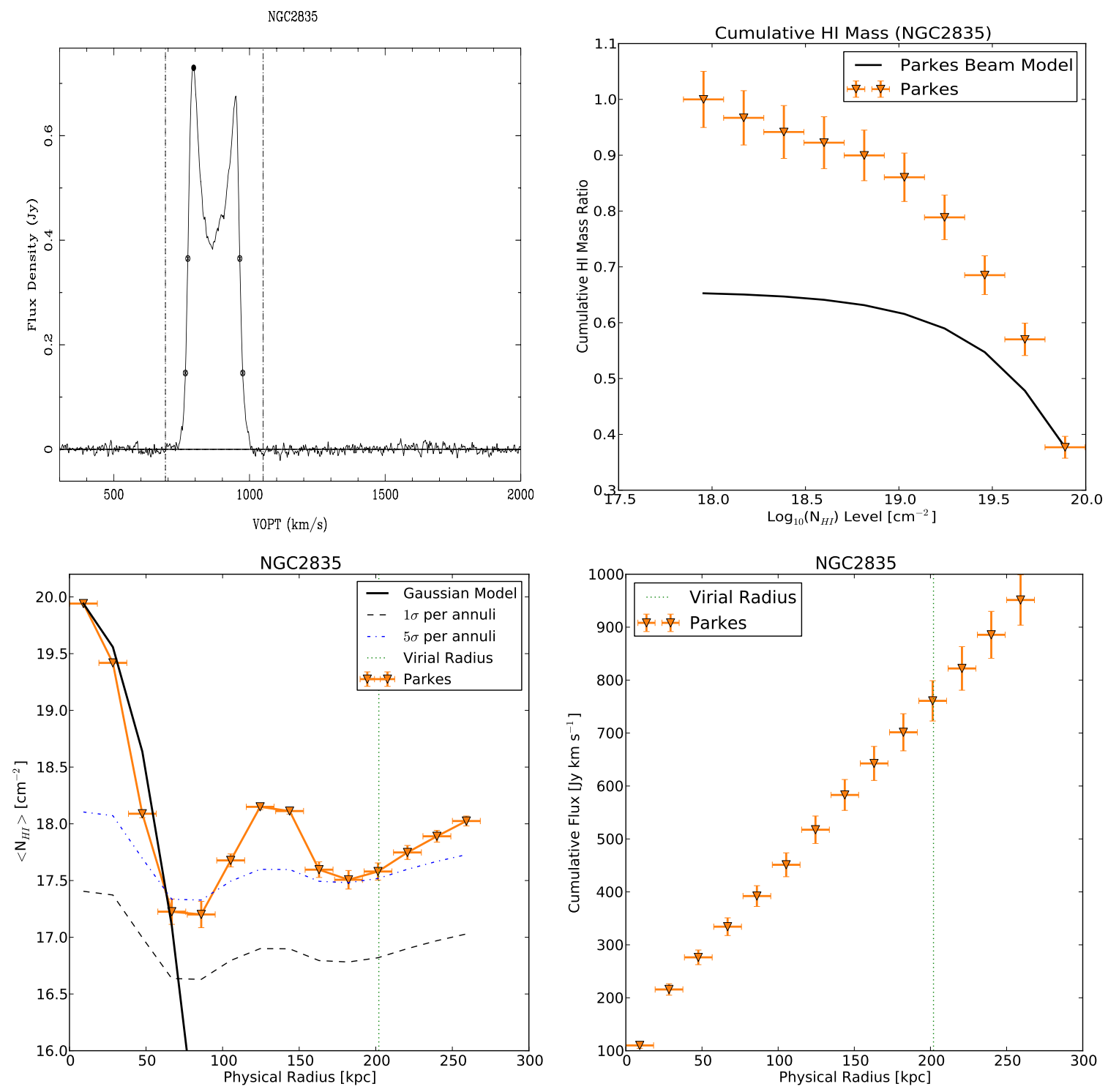

Figure 4.25: Same as Figure 4.12, but for NGC2835. 

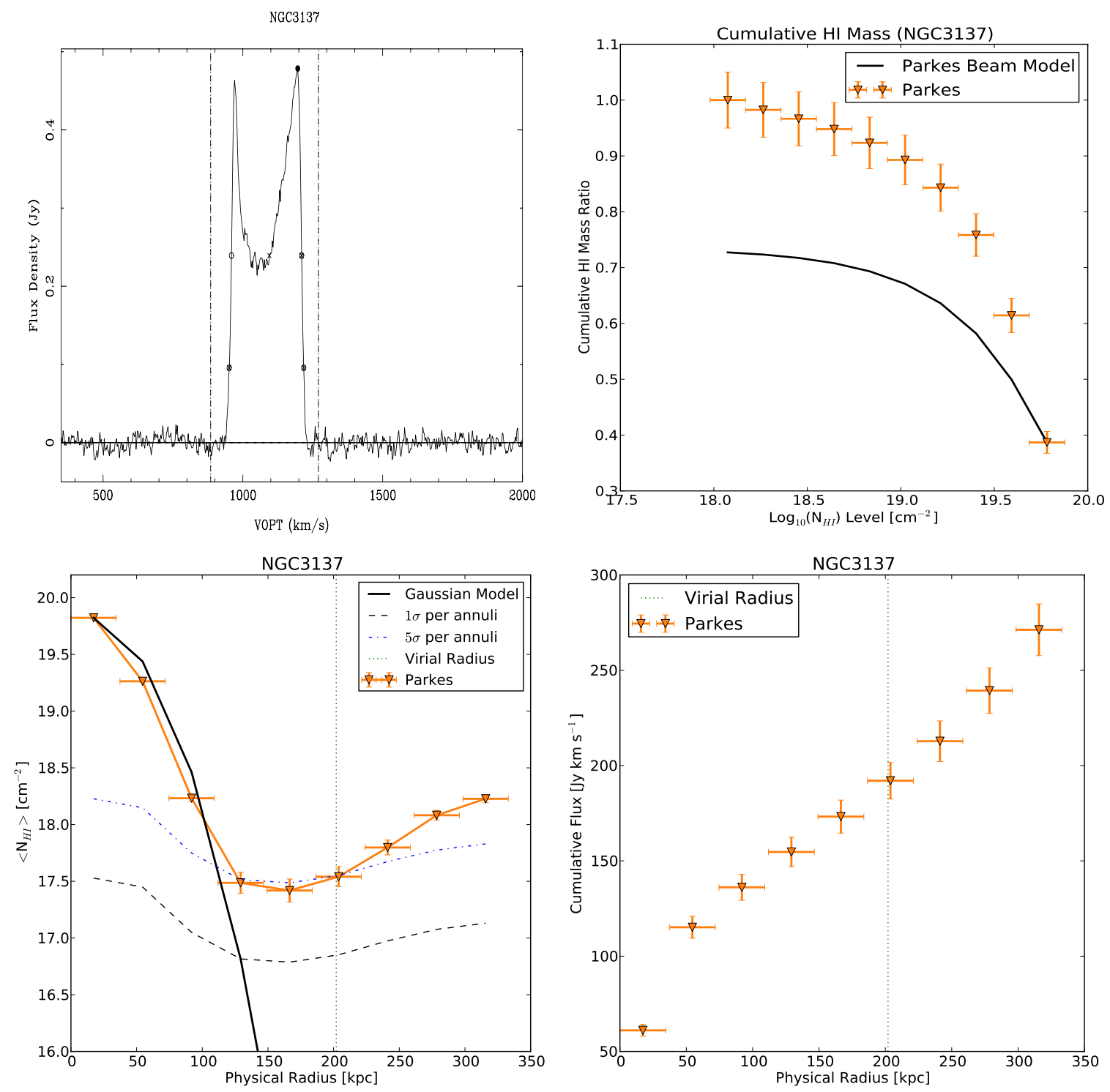

Figure 4.26: Same as Figure 4.12, but for NGC3137. 

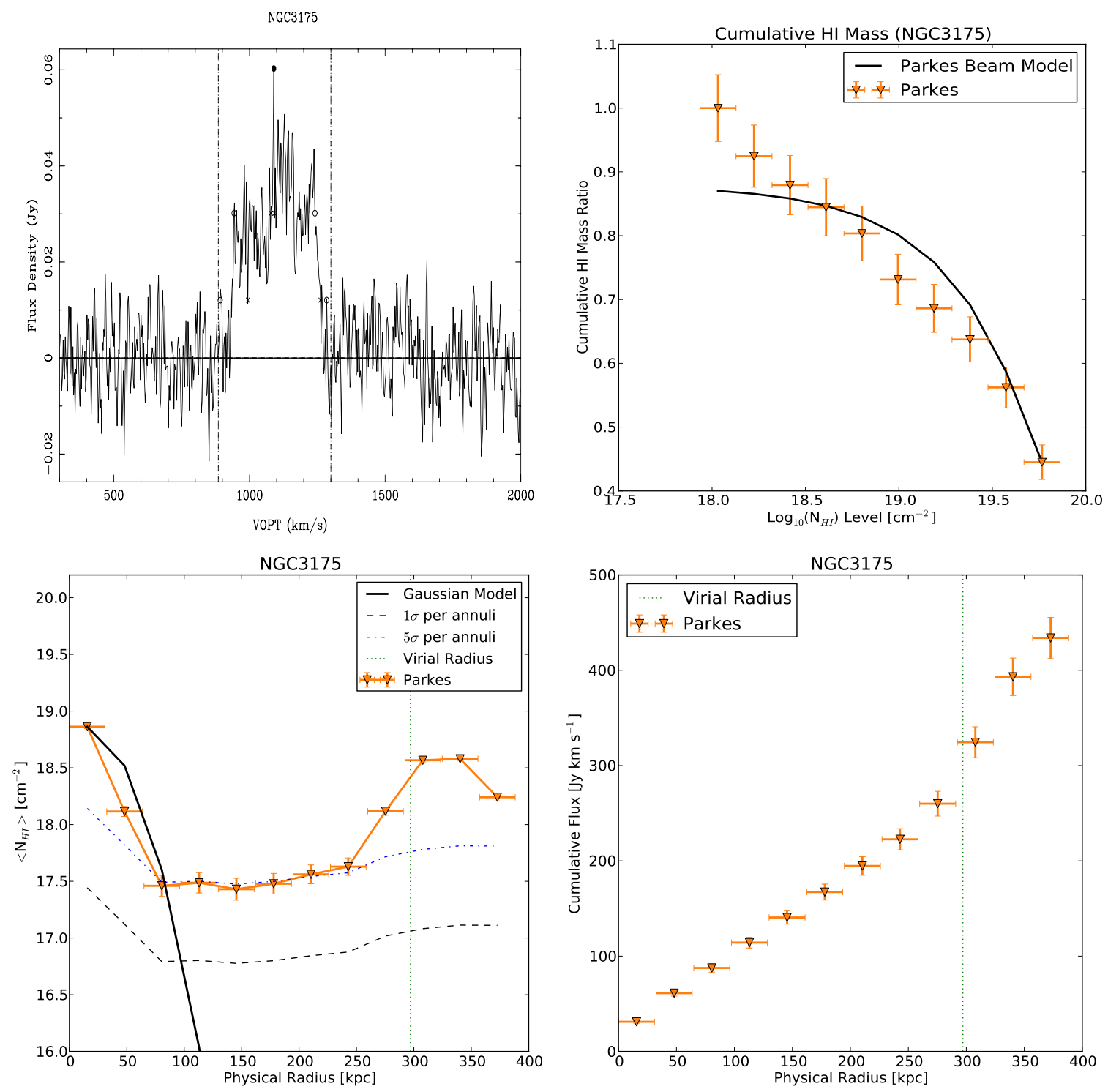

Figure 4.27: Same as Figure 4.12, but for NGC3175. 

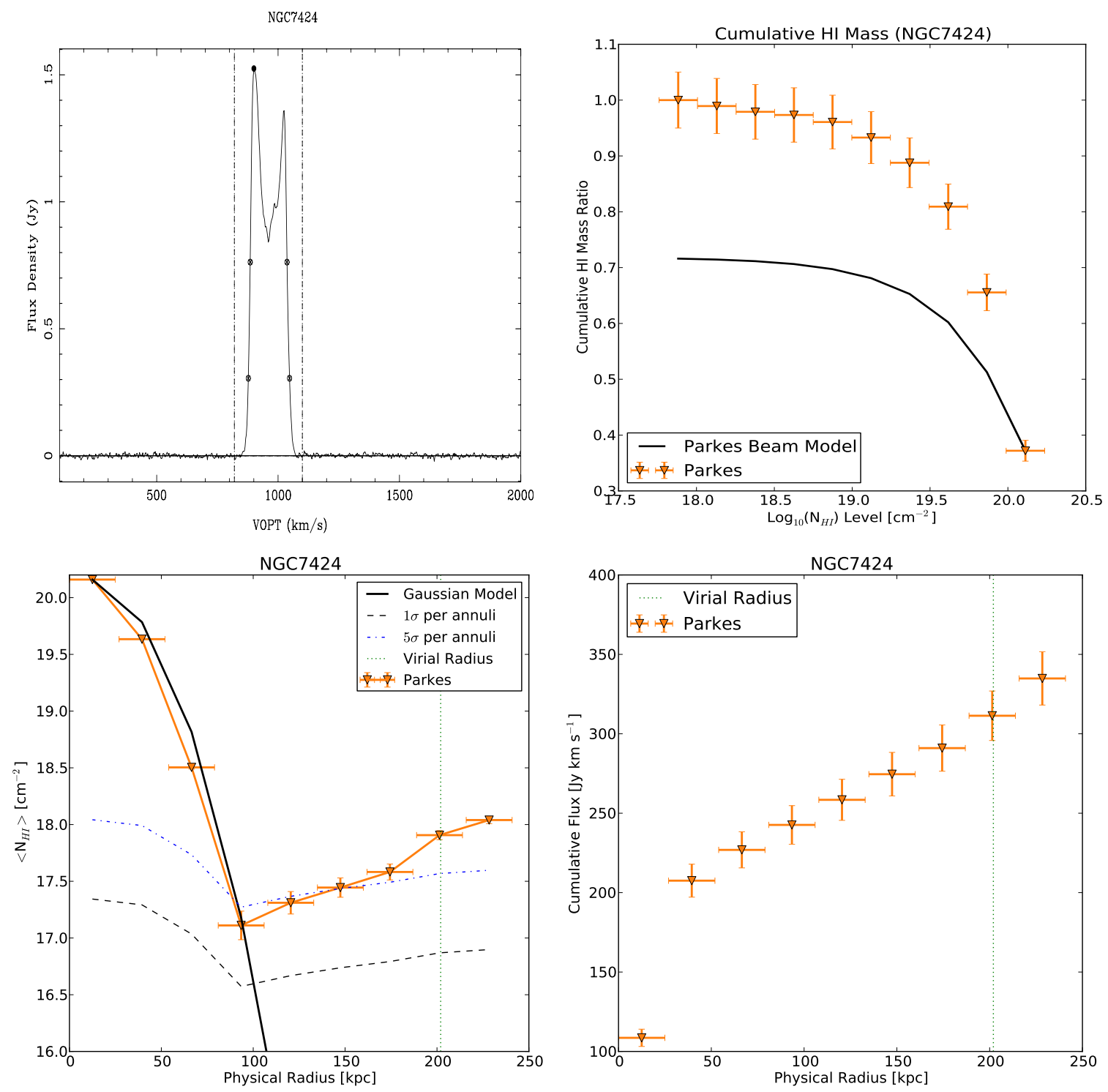

Figure 4.28: Same as Figure 4.12, but for NGC7424. 

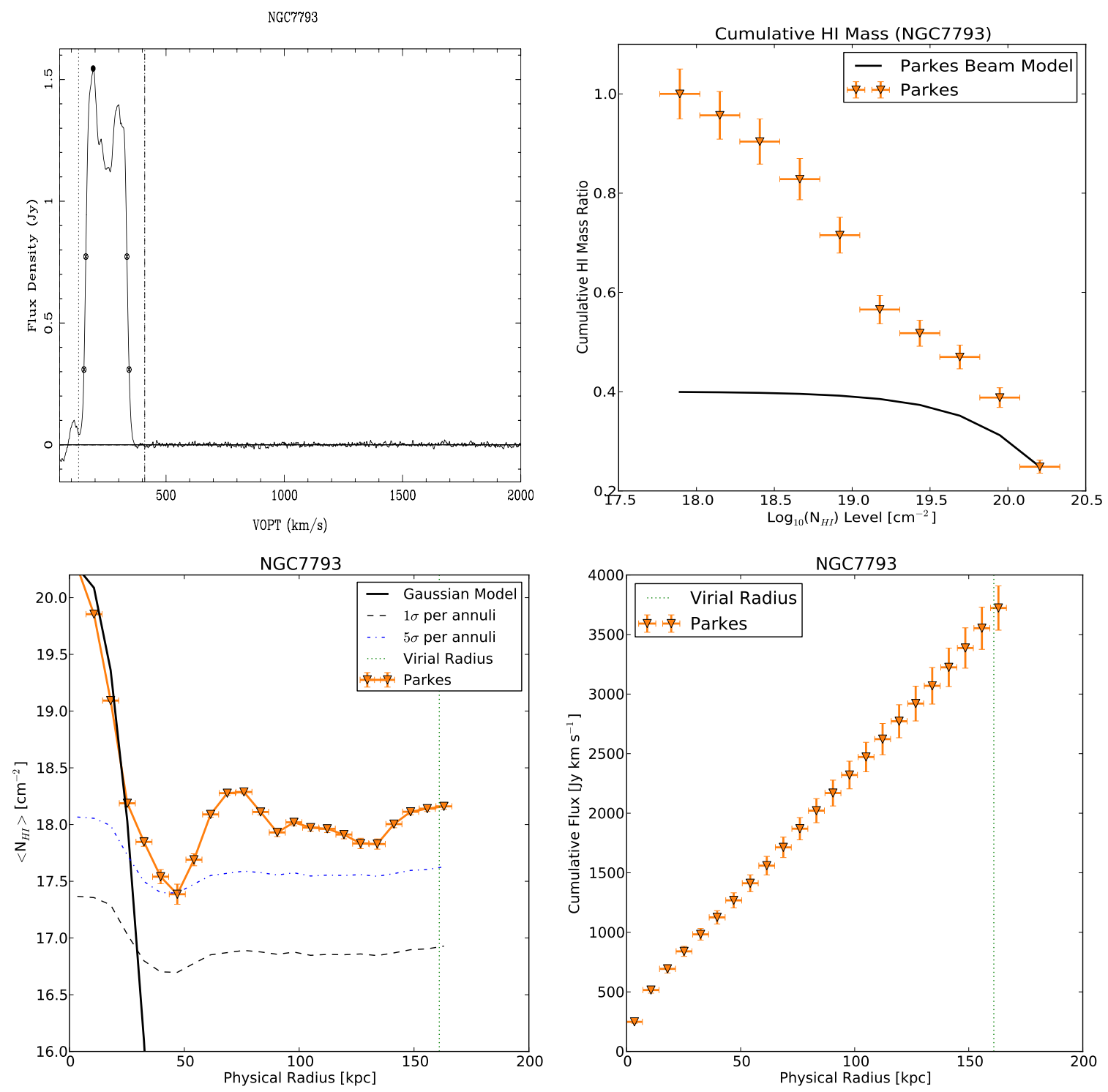

Figure 4.29: Same as Figure 4.12, but for NGC7793. 
function of column density, the azimuthally averaged column density levels in annuli out to the virial radius, and the cumulative flux summed by annuli out to the virial radius of each galaxy.

For all but one galaxy, NGC3175, the Parkes data seems to be resolved as compared to the Gaussian beam model shown in the cumulative H I mass plots. Some of our galaxies reveal an increase in the amount of diffuse gas (defined as $N_{\mathrm{HI}}<$ $10^{19} \mathrm{~cm}^{-2}$ ). This behavior is likely due to the extent of the region around each galaxy that we are measuring. The farther into the dark matter halo we map, the more diffuse gas we expect to see as this diffuse gas flows in from the IGM to fuel star formation in the galaxy.

If we look at how the galaxy responds in comparison to the Gaussian beam model at the smallest physical radii in the azimuthally averaged $N_{\mathrm{HI}}$ plots, again we will note that the galaxy usually appears to have a smaller radius than the model at a particular averaged column density. This tells us that the disk of these galaxies are not particularly well-resolved in the Parkes beam. At larger physical radii, in several of the galaxies we note the appearance of a companion, which emerges as a large positive deviation from the noise floor. The most pronounced example of this is seen in Figure 4.23 where NGC 1792 is in close proximity to two companions at nearly the same radius from NGC 1792. These companions, NGC 1808 and ESO 305-9, are approximately $125 \mathrm{kpc}$ from the center of NGC 1792. Other examples of this can be seen in Figure 4.12 (ESO 138-10) where we detect the companion ESO 138-9 around 180 kpc, Figure 4.15 (ESO214-17) where we detect ESO 264-35 around 240 kpc, well outside the virial radius, Figure 4.17 (NGC 24) where we detect another IMAGINE 
galaxy, NGC 45, at around $300 \mathrm{kpc}$, also well outside the virial radius. ESO 486-21 appears in Figure 4.22 at a distance of about $150 \mathrm{kpc}$ from NGC 1744. NGC 2835 has a companion, ESO565-1, which shows up in Figure 4.25 around $125 \mathrm{kpc}$. At the edge of NGC 7424's virial radius, part of NGC 7462 can be seen in Figure 4.28.

We summed up the total flux in our images and plotted those as a function of physical radius, where we are most interested in the region up to the virial radius. Each map demonstrates linear growth from the galaxy out to or beyond the virial radius.

\subsubsection{Diffuse H i Fraction}

Using the method laid out in Chapter 3, we define a column density level below which the H I would be considered diffuse. Again, we use $N_{\mathrm{HI}}=10^{19} \mathrm{~cm}^{-2}$ as an upper limit for diffuse H I column density. This limit is based on work by Bochkarev \& Siuniaev (1977) and Maloney (1993), who suggest that the H I in a galaxy dramatically drops at column density levels below $N_{\mathrm{HI}} \sim 10^{19} \mathrm{~cm}^{-2}$, beyond which the extragalactic radiation field ionizes the neutral hydrogen. This "edge" to the neutral hydrogen has been difficult to determine on a statistical scale due to the limitations in depth and sensitivity of previous H I surveys. We determine the fraction of diffuse $\mathrm{H}$ I in a galaxy halo with,

$$
f_{19}=1-\frac{M_{19}}{M_{\mathrm{HI}}}
$$

Here, the fraction of H I mass in the cube which falls below column density $N_{\mathrm{HI}}=$ $10^{19} \mathrm{~cm}^{-2}, f_{19}$ (Pingel et al., 2018), is defined by the ratio of H I mass above $N_{\mathrm{HI}}=$ 
$10^{19} \mathrm{~cm}^{-2}, M_{19}$ to the total H I mass, $M_{\mathrm{HI}}$. For each galaxy, a $3 \sigma$ value is determined and converted to a column density. Each pixel in the image with a value above $3 \sigma_{N_{\mathrm{HI}}}$ and below $N_{\mathrm{HI}}=10^{19} \mathrm{~cm}^{-2}\left(3 \sigma_{N_{\mathrm{HI}}}<N_{\mathrm{HI}}<10^{19} \mathrm{~cm}^{-2}\right)$ contributes to the diffuse H I mass fraction. The $f_{19}$ values in our IMAGINE observations range from $0.08<f_{19}<0.76$. These diffuse H I fractions include diffuse H I throughout the CGM of each galaxy, and therefore could include anomalous gas, H I clouds, low $N_{\mathrm{HI}}$ companion galaxies, or accretion from the IGM.

Because we are concerned more with column densities rather than the total masses of the galaxies for this analysis, we need the size of the galaxy to fill the beam in order to give us a meaningful column density value. For this reason, we want the measured H I angular area of each galaxy to be larger than the area of the Parkes beam. We determine the H I area by counting the number of pixels in a small region around each galaxy that have a column density value above $N_{\mathrm{HI}}=1 \times 10^{19} \mathrm{~cm}^{-2}$ and converting this to an area. These areas are plotted in panel (a) in Figure 4.30, where the area of the Parkes beam is indicated by the dashed vertical line defined at 1.13 FWHM $^{2}$. This plot demonstrates in one more test whether or not our sources are resolved in the Parkes beam. Here, all but two of our sources extend beyond the size of the Parkes beam, and can be considered resolved, giving us reliable values of $f_{19}$. The two unresolved sources are shown as open circles in each plot.

These values represent the diffuse H I mass in the entire virial volume of each galaxy, therefore we should see no trend when plotting $f_{19}$ against distance, as the extent of the maps varies by galaxy. Indeed, panel (b) in Figure 4.30 shows that there is no trend between distance and the diffuse H I fraction. Panel (c) shows $f_{19}$ 
versus physical area in $\mathrm{kpc}^{2}$. Sources with the lowest physical areas show a range of $f_{19}$ values, however, as the galaxies get larger, there is a slight trend downward in diffuse $\mathrm{H}$ I fractions.

We plotted the $f_{19}$ values against galaxy density for two reasons. We can check for bias in the $f_{19}$ value, since these values represent any diffuse H I within the entire virial volume of these galaxies. As the galaxy density increases, we may see an increase in the diffuse fraction if this fraction was biased toward companion-rich galaxies. Panel (d) demonstrates the lack of bias toward higher-density galaxies, so we can safely say that the presence of companions around galaxies is not a prerequisite of high diffuse HI fraction. This is consistent with results from simulations by Stevens et al. (2019), which demonstrate that "a greater fraction of satellites are devoid of HI" in higher-mass halos. Another benefit of viewing $f_{19}$ as a function of galaxy density is to compare with current models of cold mode accretion. Kereš et al. (2005) demonstrated in simulations that the cold mode accretion of gas onto galaxies dominates in low density environments, and becomes insignificant at densities above $1\left(n_{\text {gal }} \gtrsim 1 \mathrm{Mpc}^{-3}\right)$. Each of the galaxies in our sample fall into the low-density category, making them prime candidates for cold accretion, but simultaneously making it difficult to find any trends. Nevertheless, there is some indication of a trend toward higher $f_{19}$ values at the lower end of our density range. Panel (d) displays this range of galaxy densities - obtained from Tully (1988) - in our sample. Without sampling both high and low-density environments, we cannot make a comparison of $f_{19}$ in both environments. However, many of our galaxies have a significant diffuse H I fraction at the lowest densities, consistent with this picture of cold accretion dominating low 
density environments.

An additional result from Kereš et al. (2005) on cold mode dominated regimes was a strong dependency on galaxy mass, finding that accretion occurs primarily in the cold mode for galaxies below a baryonic mass of $M_{\text {bary }} \sim 10^{10.3} M_{\odot}$ or a dark matter halo mass of $M_{\text {halo }} \sim 10^{11.4} M_{\odot}$. We calculated $M_{\text {bary }}$ for each galaxy using $\mathrm{M}_{\text {bary }}=\mathrm{M}_{*}+1.36 \cdot \mathrm{M}_{\mathrm{HI}}$, where we scale the HI mass to account for neutral He. To calculate $M_{b a r y}$, we use the $M_{*}$ from Leroy et al. (in prep.). We do not see any strong trends when plotting $M_{b a r y}$ against $f_{19}$, however all of our high diffuse fractions are below the $M_{\text {bary }} \sim 10^{10.3} M_{\odot}$ threshold, making this result consistent with cold accretion. We calculated the dark matter halo masses of each galaxy using the stellar-to-halo mass relation from Moster et al. (2010) and have plotted those against $f_{19}$. Here we do not see any trends in halo mass below $M_{\text {halo }} \sim 10^{11.4} M_{\odot}$ in this comparison.

Kannappan et al. (2013) showed that galaxies are bimodal at the transition to being gas-dominated, with a gas-richness threshold scale of $V_{\text {rot }} \sim 125 \mathrm{~km} \mathrm{~s}^{-1}$, where gas fractions increase. This threshold indicates a velocity below which galaxies are refueled, and become gas-rich. Again, we do not see any clear trends when we compare our rotation velocities, $V_{\text {rot }}$, to $f_{19}$ in panel $(\mathrm{g})$.

As cold mode accretion fuels star formation, and dominates in lower mass galaxies, we want to know how the current star formation rate scales relative to its stellar mass, or the specific star formation rate (sSFR). These sSFR values are calculated using the SFR and $M_{*}$ values from Leroy et al. (in prep.), and as star formation and galaxy mass is directly related to cold accretion, we would expect to see higher $f_{19}$ 

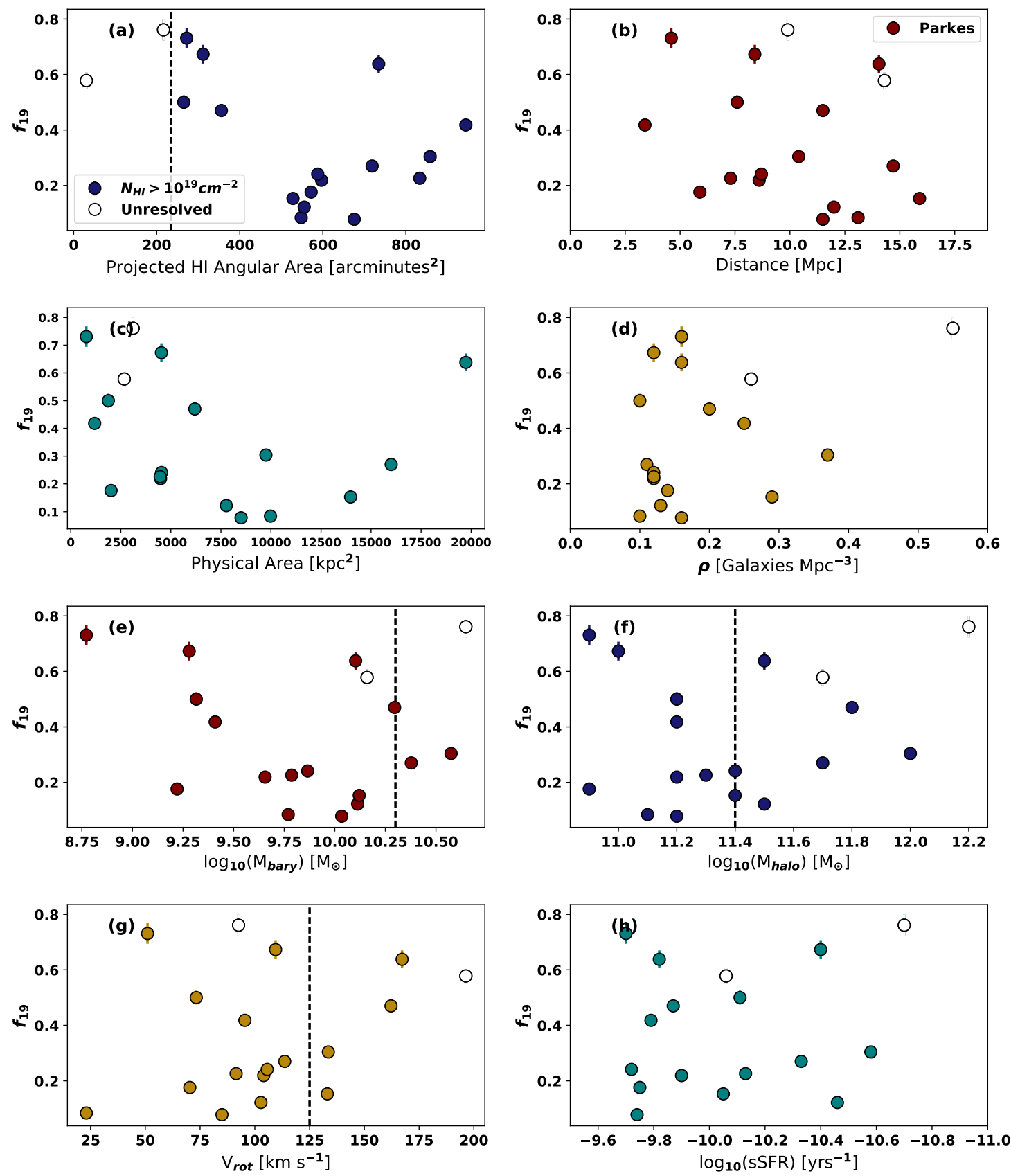

Figure 4.30: Comparisons of the diffuse fraction of $\mathrm{H} \mathrm{I}, f_{19}$, in the virial volume of each IMAGINE galaxy in our sample with: (a) Angular areas calculated inside region where column densities reach $N_{\mathrm{HI}}>5 \times 10^{18} \mathrm{~cm}^{-2}$ and $N_{\mathrm{HI}}>1 \times 10^{19} \mathrm{~cm}^{-2}$, for comparison. Vertical dashed line represents the angular area of the 14.4' FWHM Parkes beam. (b) Distance. (c) Physical areas calculated from the $N_{\mathrm{HI}}=5 \times 10^{18} \mathrm{~cm}^{-2}$ angular diameter. (d) Galaxy density. (e) Baryonic mass using $\mathrm{M}_{\text {bary }}=1.36 \cdot \mathrm{M}_{\mathrm{HI}}+\mathrm{M}_{*}$. Vertical dashed line at the threshold given in Kereš et al. (2005). (f) H I mass in the disk from this work. (g) Rotation velocity using $\mathrm{V}_{\text {rot }}=\mathrm{W}_{20} / 2$ (h) Specific star formation rate derived using SFR from Leroy et al. (in prep.). 
values associated with higher sSFR values. The last comparison we make in Figure 4.30 is of the sSFR vs. $f_{19}$ in panel (h). Once again, we see no obvious trend in our values of $f_{19}$.

\subsection{Discussion}

\subsubsection{How much H I mass is in the CGM?}

If we compare the total amount of H I mass that we detected throughout the virial volume of each galaxy with the total integrated H I in the disk obtained from the spectra, we can quantify the amount of H I in the CGM alone. H I masses in the disk of each galaxy were derived by integrating the detected flux values in the profile of the galaxy over the velocity range of that profile. We calculated the total H I mass in the dark matter halo, by summing up the pixels within the virial radius. The H I mass in the CGM is obtained by subtracting the H I disk mass from the H I halo mass. In many cases, the CGM mass is very near zero. In some of these cases the value is negative. This is caused by variations in the baselining of the data. We calculated the fraction of H I mass in the CGM in terms of the H I mass in the halo using:

$$
f_{C G M}=\frac{M_{\mathrm{HI}, \text { halo }}-M_{\mathrm{HI}, \text { disk }}}{M_{\mathrm{HI}, \text { halo }}},
$$

where $f_{C G M}$ describes the fraction of the H I halo that lies exclusively in the CGM. If the CGM of a galaxy contained no extraplanar H I (outside of the H I disk of the galaxy), this fraction would plainly be zero. For most of our galaxies, the H I disk 


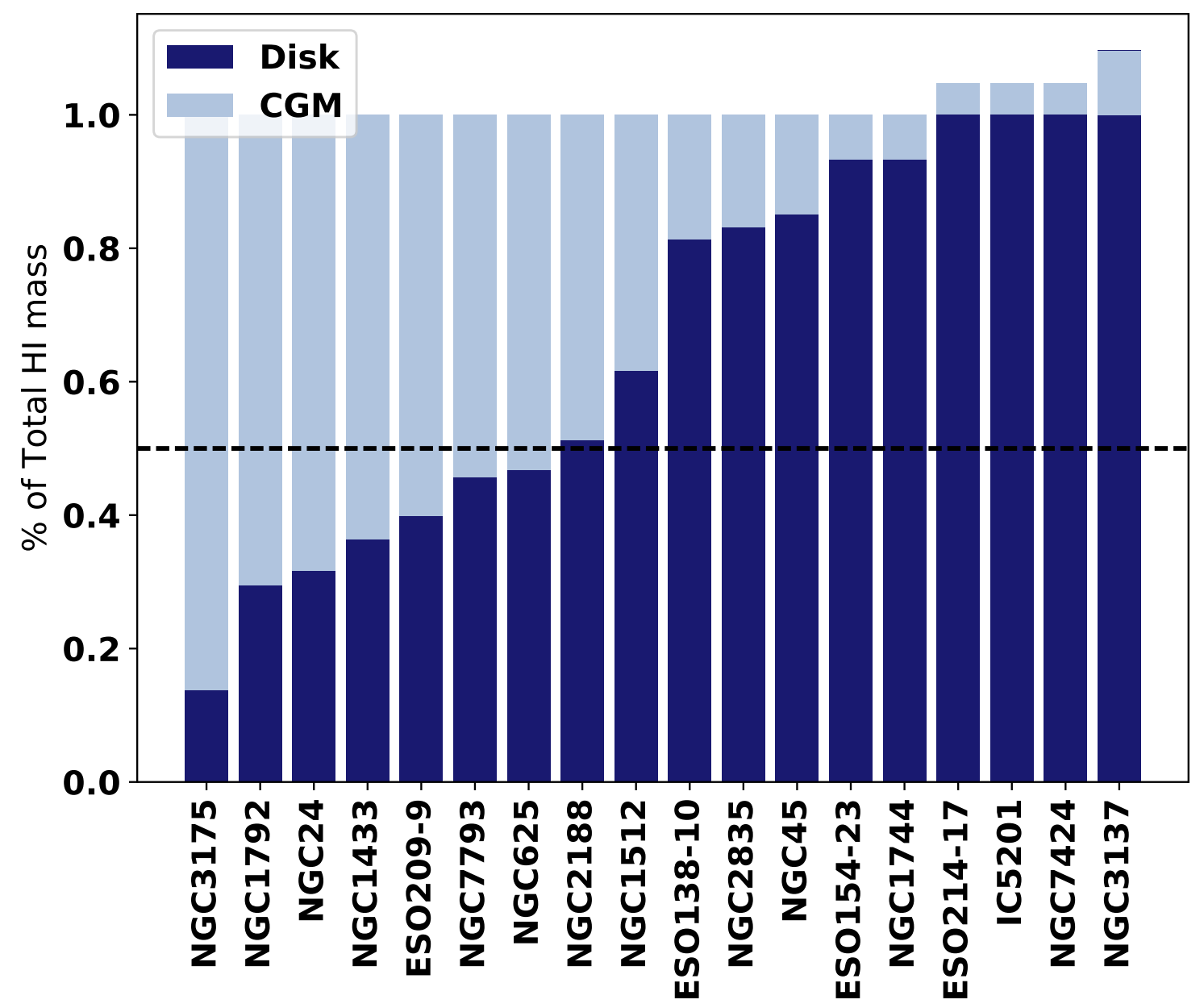

Figure 4.31: Histogram of H I halo masses in our IMAGINE sample. The H I in the entire dark matter halo, or the virial volume, is represented by the H I mass in the disk of the galaxy, and the H I mass in the CGM. These are separated by percentage of the H I halo that each occupies. The dashed line represents half of the H I mass in the dark matter halo.

makes up more than half of the HI halo mass. These disk-dominated halos make up eleven of our sample. The halos of seven of our galaxies hold more H I mass in their CGM than in their disks. The percentages showing disk-dominated and CGMdominated galaxies can be seen in Figure 4.1, where the sources with a fraction of the total mass above one in the four completely disk-dominated sources again come from differences in methods for baseline subtraction. 
We also wanted to do the same comparison with $f_{C G M}$ that we did with $f_{19}$ in multiple environments that favor cold mode accretion. These plots show our range of values for $f_{C G M}$, ranging from zero, meaning there is no additional $\mathrm{H}$ I mass in the CGM, up to $\sim 86 \%$ of the halo made up by CGM H I. The comparisons with various environmental properties are shown in Figure 4.32. We do not see any new trends here when comparing the CGM fraction of the halo with these environments.

\subsubsection{Is there enough CGM mass to sustain star formation?}

We can answer the question of whether or not these galaxies have enough gas mass to sustain star formation by calculating the timescale in which each galaxy would run out of gas, given a constant rate of star formation. This gas depletion timescale is a simple calculation, using $\tau_{d e p}^{g a s}=\frac{M_{g a s}}{S F R}$. Here, $M_{g a s}$ refers to the total amount of gas (neutral and molecular) available to form stars, and $\tau_{d e p}^{g a s}$ tells us how long it will take for that galaxy to consume the gas. Since the typical H I observation of a galaxy is of the disk rather than the entire virial volume, many H I depletion timescales refer to this amount of gas only. We will compare the depletion timescale of the $\mathrm{H}$ I disk with the time it would take for the galaxy to consume the entire amount of gas in the halo, assuming that all the neutral gas in the CGM is accreted onto the galaxy, and that the galaxy is $100 \%$ efficient in converting its neutral gas to stars. 

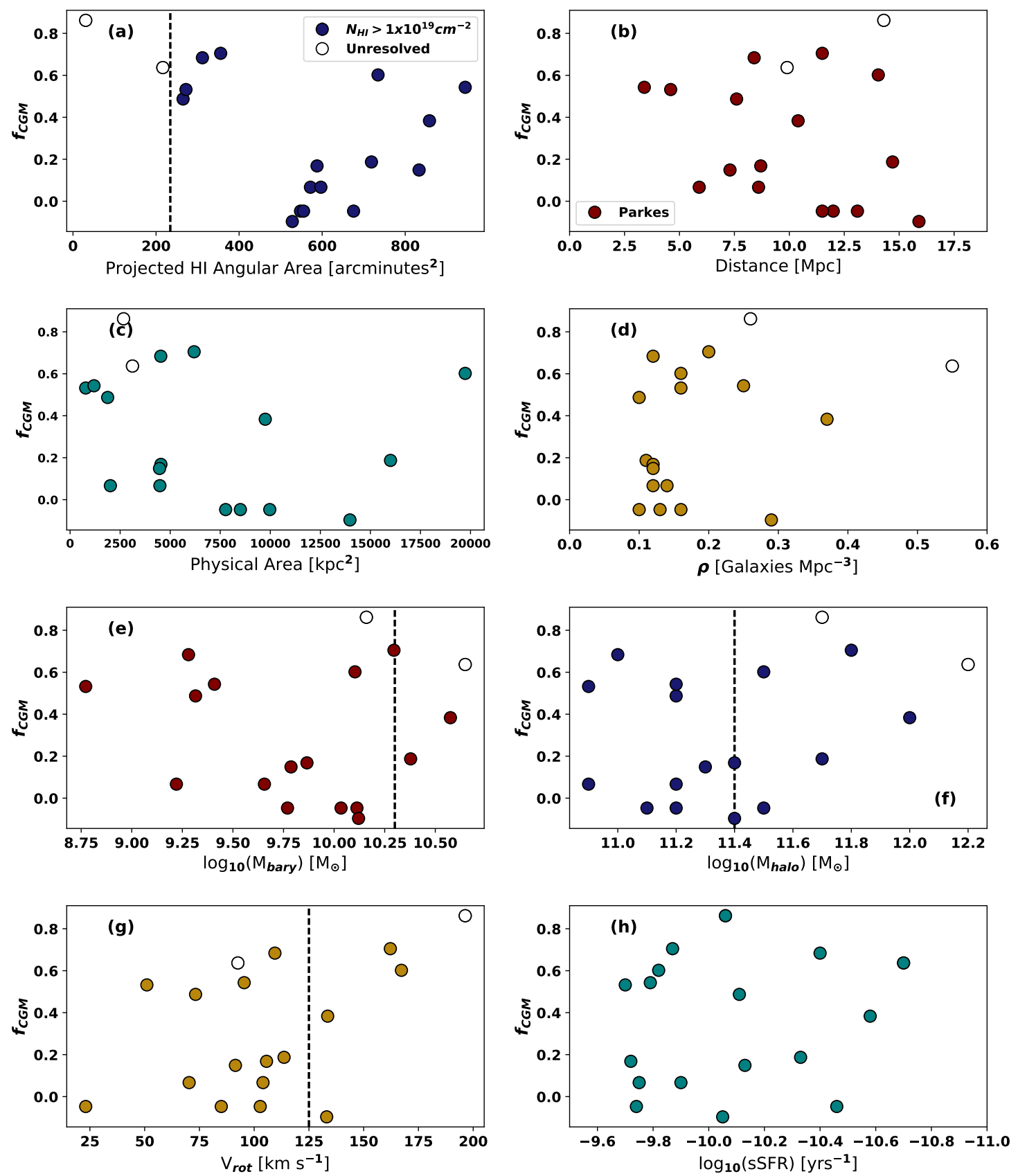

Figure 4.32: Same as Figure 4.30, but for comparisons with the fraction of total H I gas in the dark matter halo residing exclusively in the CGM, $f_{C G M}$. 
Table 4.4: Depletion timescales of the disk and halo

\begin{tabular}{|c|c|c|c|c|c|c|c|}
\hline Source & $\begin{array}{c}\mathrm{SFR} \\
{\left[M_{\odot} \mathrm{yr}^{-1}\right]} \\
(2)\end{array}$ & $\begin{array}{c}\log M_{\text {disk }}^{\mathrm{HI}} \\
{\left[M_{\odot}\right]} \\
(3)\end{array}$ & $\begin{array}{c}\log M_{\text {halo }}^{\mathrm{HI}} \\
{\left[M_{\odot}\right]} \\
(4)\end{array}$ & $\begin{array}{c}\tau_{d i s k}^{g a s} \\
{[\mathrm{Gyr}]} \\
(5)\end{array}$ & $\begin{array}{c}\tau_{\text {halo }}^{\text {gas }} \\
{[\mathrm{Gyr}]} \\
(6)\end{array}$ & $f_{19}$ & $f_{C G M}$ \\
\hline ESO138-10 & 0.646 & 9.87 & 9.96 & 15.61 & 19.21 & 0.27 & 0.19 \\
\hline ESO154-23 & 0.054 & 9.0 & 9.03 & 25.32 & 27.14 & 0.18 & 0.07 \\
\hline ESO209-9 & 1.0 & 9.65 & 10.05 & 6.07 & 15.26 & 0.64 & 0.6 \\
\hline ESO214-17 & 1.0 & 9.57 & 9.55 & 5.05 & 4.83 & 0.08 & -0.05 \\
\hline IC5201 & 0.224 & 9.68 & 9.66 & 29.08 & 27.77 & 0.12 & -0.05 \\
\hline NGC1433 & 0.871 & 8.77 & 9.21 & 0.92 & 2.53 & 0.76 & 0.64 \\
\hline NGC1512 & 0.813 & 9.69 & 9.9 & 8.19 & 13.29 & 0.3 & 0.38 \\
\hline NGC1744 & 0.195 & 9.34 & 9.37 & 15.26 & 16.35 & 0.22 & 0.07 \\
\hline NGC1792 & 2.455 & 9.06 & 9.59 & 0.64 & 2.16 & 0.47 & 0.7 \\
\hline NGC2188 & 0.112 & 8.66 & 8.95 & 5.54 & 10.8 & 0.5 & 0.49 \\
\hline NGC24 & 0.031 & 8.92 & 9.42 & 36.6 & 115.75 & 0.67 & 0.68 \\
\hline NGC2835 & 0.832 & 9.34 & 9.42 & 3.58 & 4.3 & 0.24 & 0.17 \\
\hline NGC3137 & 0.447 & 9.78 & 9.74 & 18.35 & 16.73 & 0.15 & -0.1 \\
\hline NGC3175 & 1.202 & 8.67 & 9.53 & 0.53 & 3.83 & 0.58 & 0.86 \\
\hline NGC45 & 0.17 & 9.45 & 9.52 & 22.57 & 26.52 & 0.23 & 0.15 \\
\hline NGC625 & 0.068 & 8.27 & 8.6 & 3.75 & 8.01 & 0.73 & 0.53 \\
\hline NGC7424 & 0.372 & 9.81 & 9.79 & 23.63 & 22.57 & 0.08 & -0.05 \\
\hline NGC7793 & 0.263 & 8.84 & 9.18 & 3.58 & 7.83 & 0.42 & 0.54 \\
\hline
\end{tabular}

Table 4.4: Star formation rates obtained from Leroy et al. (in prep). Column 3 lists the H I mass of the disk. Column 4 lists the total H I mass within the virial volume. Columns 5 and 6 list the gas depletion timescales for the disk gas only, and for the $\mathrm{HI}$ in the entire virial volume of the galaxy, respectively. Column 7 is the diffuse neutral fraction and column 8 is the fraction of the $\mathrm{HI}$ halo that resides in the CGM. Negative $f_{C G M}$ values are cases in which the spectrally measured H I mass in the disk was slightly larger than the total cumulative H I mass measured in the moment 0 map, likely due to variation in baselining. 
We calculate the depletion timescale of the disk with:

$$
\tau_{d i s k}^{g a s}=\frac{M_{d i s k}^{g a s}}{S F R}
$$

where $M_{\text {disk }}^{\text {gas }}$ is the H I mass corrected for helium by a factor of 1.36 . We note that this includes only neutral gas, not molecular gas, such as $H_{2}$. We also calculate the depletion timescale using all of the detected H I within the virial volume with:

$$
\tau_{\text {halo }}^{\text {gas }}=\frac{M_{\text {halo }}^{\text {gas }}}{S F R}
$$

With this depletion timescale, we can see how long it will take the galaxy to consume all of the gas in the dark matter halo. A comparison of both the disk and the halo depletion timescales can be seen in Table 4.4 .

For 10 of our 18 galaxies, the amount of gas in the disk is insufficient to sustain star formation, where $\tau_{\text {disk }}^{\text {gas }}$ is less than a Hubble time. Of those galaxies, two contain enough gas in the halo to sustain star formation for well over a Hubble time. The disk gas depletion timescales for eight of our galaxies, however, are long enough to sustain star formation over a Hubble time, and this value mostly increases when including the gas in the halo. These larger depletion timescales are consistent with the H I those found by both Bigiel et al. (2010) and Roychowdhury et al. (2014). 


\subsection{Summary}

In this chapter, we explored the neutral hydrogen throughout the dark matter halo of 18 IMAGINE galaxies. We used the Parkes Radio Telescope to map the 21-cm line of neutral hydrogen out to the virial radius of these 18 galaxies, reaching column

density sensitivities of $N_{\mathrm{HI}}=10^{16.15-16.62} \mathrm{~cm}^{-2}$ per channel, and H I mass sensitivities of $M_{\mathrm{HI}}=10^{4.56-6.15} M_{\odot}$.

1. We quantified the total amount of $\mathrm{HI}$ detected in the virial volume of each galaxy, obtaining measurements of the disk, CGM, and the entire halo. We found that 11 of our galaxies are disk-dominated, containing more $\mathrm{H} \mathrm{I}$ in the disk than in their CGM. The remaining 7 galaxies are CGM-dominated, where over half of the HI mass in the halo is made up of H I in the CGM.

2. We calculated the fraction of diffuse neutral hydrogen throughout the halo by summing the H I mass that fell below a column density limit of $N_{\mathrm{HI}}=10^{19} \mathrm{~cm}^{-2}$. The fraction of diffuse $\mathrm{H}$ in the galaxies' halos ranged from 0.08 to 0.76 . We compared this diffuse fraction to a number of galaxy properties theorized to be associated with cold mode accretion. We found a slight trend when comparing the baryonic mass with the diffuse fraction, where high $f_{19}$ values were all associated with $M_{\text {bary }} \sim 10^{10.3} M_{\odot}$. We did not find any other strong correlations between the diffuse fraction and the other properties of cold mode accretion. Addition of the full sample of IMAGINE galaxies may reveal stronger correlations to cold mode accretion properties, or potentially tell us that this diffuse neutral fraction is not an ideal indicator of galaxies undergoing cold mode ac- 
cretion.

3. We did the same analysis on the fraction of $\mathrm{H}$ I found in the CGM, which was in the range of 0.0 to 0.86 . Again, we did not see any new correlation between this CGM fraction and the same environmental properties mentioned above. The addition of the 10 other galaxies in our sample could potentially enhance any underlying trends that were not revealed in this subsample.

4. We derived the gas depletion timescale for both the disk gas and the halo gas by using the helium-corrected H I mass in each, along with their star formation rates. We found that the amount of neutral gas in the disk was not sufficient to sustain star formation in 10 of the galaxies, where their disk depletion timescales were less than a Hubble time. When calculated for the entire amount of neutral gas in the halo, we found that 10 of the galaxies contained enough neutral gas to sustain star formation.

We will continue to complete the picture of the H I throughout the dark matter halos of spiral galaxies once we have completed Parkes maps of all 28 of the IMAGINE galaxies. With this complete data set in hand, we will be able to combine the data from the ATCA observations for a more complete sampling of baselines, revealing the connections of the H I in the CGM to the detailed distribution of H I near the disk with the shorter baselines ATCA will provide. 


\section{Chapter 5}

\section{Conclusions}

\subsection{Main Conclusions}

In the previous chapters, we have discussed results from some of the deepest H I surveys of nearby galaxies. These deep H I surveys inform us about the environments which enable galaxies to continue to form stars. We know that in order to probe the diffuse gas that flows into galaxy disks via cold mode accretion, we need to reach column density sensitivities below $N_{\mathrm{HI}} \sim 10^{19} \mathrm{~cm}^{-2}$. In Figure 5.1, we can see how the MHONGOOSE-GBT and IMAGINE-Parkes surveys compare with the sensitivities of other H I surveys. Our IMAGINE observations have reached the deepest detection sensitivities of any other H I surveys, with the exception of the GBT observations of M31 (Wolfe et al., 2016).

One of these deep Parkes maps revealed a new H I bridge between IMAGINE galaxy ESO 214-17 and a satellite, ESO 214-18. This bridge appears to be a smooth, continuous distribution of $\mathrm{H}$ I which peaks at a $20 \sigma$ column density detection of $N_{\mathrm{HI}}=$ $2.7 \times 10^{17} \mathrm{~cm}^{-2}$. We mapped well beyond the virial radius of ESO 214-17, yielding detections of both ESO 264-35 and a potentially new H I detection of edge-on, spiral galaxy, ESO 214-15.

We also used the GBT to map 18 MHONGOOSE galaxies down to $3 \sigma$ column density levels of $N_{\mathrm{HI}} \sim 10^{18} \mathrm{~cm}^{-2}$ over a $16 \mathrm{~km} \mathrm{~s}^{-1}$ linewidth. We measured the amount 


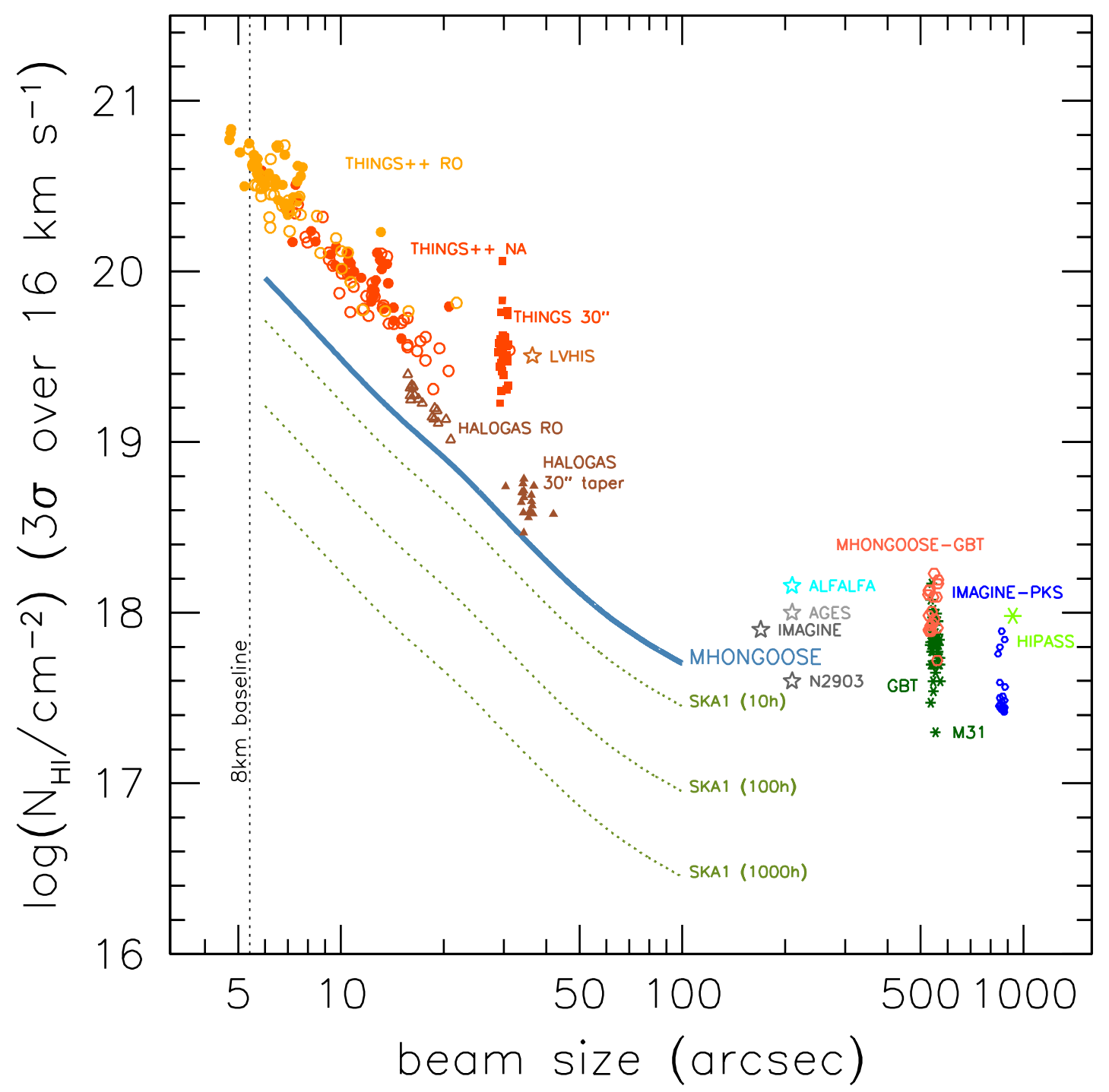

Figure 5.1: H I column density sensitivities at the $3 \sigma$ detection level, over a linewidth of $16 \mathrm{~km} \mathrm{~s}^{-1}$. The two surveys presented here are marked with open coral circles for the MHONGOOSE-GBT survey, and with open blue circles for the completed galaxies in the IMAGINE-PKS survey. Image credit: de Blok et al. (2016). 
of diffuse H I present in each galaxy, where we defined diffuse as $\mathrm{H}$ I at $N_{\mathrm{HI}}$ levels below $10^{19} \mathrm{~cm}^{-2}$. Of our 18 observed galaxies, 11 were made up of less than $50 \%$ diffuse H I, while the rest contained between 50 and 93\% diffuse H I. Considering that diffuse gas is believed to be fed directly from the IGM, or the cosmic web, into the disks of galaxies, we examined these diffuse H I fractions in relation to properties related to cold mode accretion from the IGM. The results of these comparisons support current correlations to cold mode accretion, such as higher amounts of diffuse gas in galaxies with baryonic masses below $10^{10.3} M_{\odot}$, dark matter halo masses below $10^{11.4} M_{\odot}$, and rotation velocities below $125 \mathrm{~km} \mathrm{~s}^{-1}$. Our highest diffuse fractions are also associated with higher specific star formation rates. We also calculated gas depletion timescales for each galaxy that we had star formation rates for, revealing that all but two galaxies contained enough gas to sustain star formation for well over a Hubble time.

We made maps of 18 IMAGINE galaxies with the Parkes radio telescope, encompassing the entire virial volume of each galaxy. This is the first survey to probe the entire dark matter halo of nearby galaxies down to the extremely low column densities. With these maps, we were able to quantify the total amount of $\mathrm{H}$ I in both the disk and the CGM of each galaxy. This allowed us to determine which galaxies are disk-dominated, and which are CGM gas-dominated, with 11 galaxies being diskdominated and seven CGM gas-dominated. We also measured the diffuse H I fraction in these galaxies and compared this to the properties and environments we expect to find cold accretion. We find only weak correlation between high diffuse fraction and those properties expected in cold mode accretion. The same analysis done with the fraction of H I residing in the CGM resulted in no correlation. With both the amount 

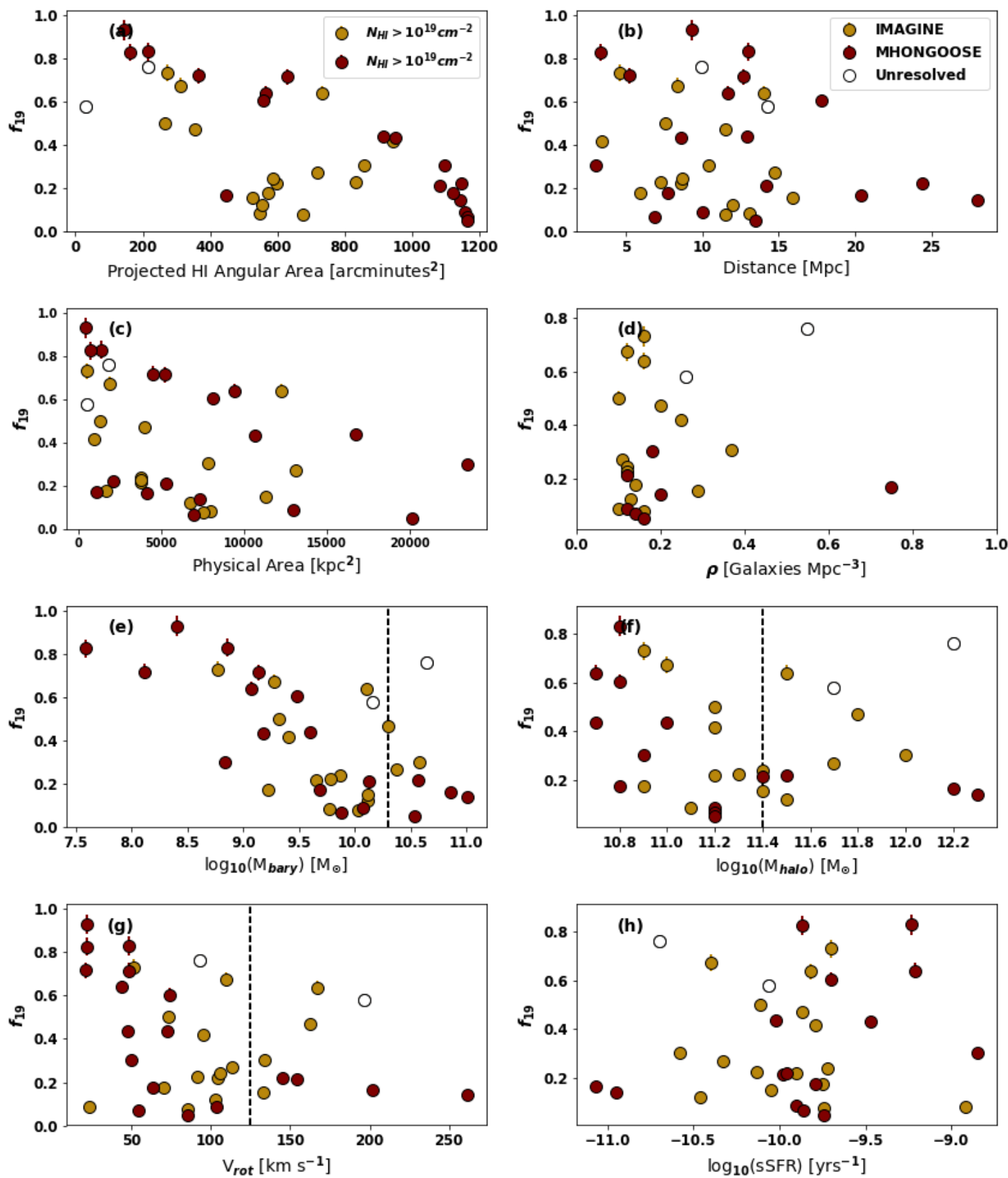

Figure 5.2: Comparisons of the diffuse fraction of $\mathrm{HI}$ in the virial volume of each IMAGINE and MHONGOOSE galaxy in presented in this work: (a) Angular areas calculated inside region where column densities reach $N_{\mathrm{HI}}>5 \times 10^{18} \mathrm{~cm}^{-2}$ and $N_{\mathrm{HI}}>$ $1 \times 10^{19} \mathrm{~cm}^{-2}$, for comparison. Vertical dashed line represents the angular area of the 14.4' FWHM Parkes beam. (b) Distance. (c) Physical areas calculated from the $N_{\mathrm{HI}}=5 \times 10^{18} \mathrm{~cm}^{-2}$ angular diameter. (d) Galaxy density. (e) Baryonic mass using $\mathrm{M}_{\text {bary }}=1.36 \cdot \mathrm{M}_{\mathrm{HI}}+\mathrm{M}_{*}$. Vertical dashed line at the threshold given in Kereš et al. (2005). (f) H I mass in the disk from this work. (g) Rotation velocity using $\mathrm{V}_{\text {rot }}=\mathrm{W}_{20} / 2(\mathrm{~h})$ Specific star formation rate derived using SFR from Leroy et al. (in prep.). 
of H I mass in the disk and throughout the entire halo, we calculated separate gas depletion timescales for the disk and the halo gas. Over half of the galaxies did not contain enough gas to sustain star formation, while that number was reversed when accounting for all the neutral gas in the halo, where over half the galaxies did contain enough gas to sustain star formation.

Here, we compare the environmental analysis mentioned previously, with the entire sample of galaxies in this thesis. Figure 5.2 shows both the MHONGOOSE and IMAGINE sample presented here. Viewing the entire sample of 36 galaxies reveals a stronger correlation between the diffuse $\mathrm{H}$ I fraction and properties of cold mode accretion, specifically in galaxy density, baryonic mass, dark matter halo mass, and rotation velocity. The strongest trends we see here include the physical area in panel (c), the baryonic mass in panel (e), the dark matter halo mass in panel (f), and the rotation velocity in panel $(\mathrm{g})$.

\subsection{Future Plans}

In the future, I plan to complete the sample of IMAGINE galaxies with Parkes, creating a full, unbiased sample of galaxies, which will inform us on the statistical properties of the CGM. Each of these maps will also be combined with the ATCA maps of all IMAGINE galaxies, enabling us to get a complete picture of the distribution of H I at all spacial scales. We will continue to search for evidence of cold mode accretion in these galaxies with the complete, combined maps of IMAGINE galaxies.

Additionally, I plan to investigate the transition HI makes as it moves from 
large-scale H I flows from the IGM, to the accretion onto the disk through the dark matter halo, and the process it takes as it condenses into molecular hydrogen to form stars. The physics of this process is still unknown and the connection of these mechanisms is an exciting and puzzling area of astronomy yet to be uncovered. 


\section{Bibliography}

Barnes, D. G., Staveley-Smith, L., de Blok, W. J. G., et al. 2001, MNRAS, 322, 486

Bell, E. F., \& de Jong, R. S. 2001, ApJ, 550, 212

Bell, E. F., Naab, T., McIntosh, D. H., et al. 2006, ApJ, 640, 241

Bernard, E. J., Ferguson, A. M. N., Barker, M. K., et al. 2012, MNRAS, 420, 2625

Bigiel, F., Leroy, A., Walter, F., et al. 2010, The Astronomical Journal, 140, 1194

-. 2008, AJ, 136, 2846

Bigiel, F., Leroy, A. K., Walter, F., et al. 2011, ApJL, 730, L13

Binney, J., \& Tremaine, S. 2008, Galactic Dynamics: Second Edition

Birnboim, Y., \& Dekel, A. 2003, MNRAS, 345, 349

Bland-Hawthorn, J., Maloney, P. R., Stephens, A., Zovaro, A., \& Popping, A. 2017, ApJ, 849, 51

Bochkarev, N. G., \& Siuniaev, R. A. 1977, , 21, 542

Bond, J. R., Kofman, L., \& Pogosyan, D. 1996, Nature, 380, 603

Boothroyd, A. I., Blagrave, K., Lockman, F. J., et al. 2011, A\&A, 536, A81

Braun, R., \& Thilker, D. A. 2004, A\&A, 417, 421

Broeils, A. H., \& Rhee, M.-H. 1997, A\&A, 324, 877 
Cantalupo, S., Arrigoni-Battaia, F., Prochaska, J. X., Hennawi, J. F., \& Madau, P. 2014, Nature, 506, 63

Cen, R., \& Ostriker, J. P. 1999, ApJ, 514, 1

Cole, S., Lacey, C. G., Baugh, C. M., \& Frenk, C. S. 2000, MNRAS, 319, 168

Crighton, N. H. M., Murphy, M. T., Prochaska, J. X., et al. 2015, MNRAS, 452, 217

Daddi, E., Bournaud, F., Walter, F., et al. 2010, ApJ, 713, 686

Dahari, O. 1984, AJ, 89, 966

Davé, R., Hernquist, L., Katz, N., \& Weinberg, D. H. 1999, ApJ, 511, 521

Davé, R., Cen, R., Ostriker, J. P., et al. 2001, ApJ, 552, 473

Davis, M., Efstathiou, G., Frenk, C. S., \& White, S. D. M. 1985, ApJ, 292, 371

de Blok, W. J. G., Adams, E. A. K., Amram, P., et al. 2016, in Proceedings of MeerKAT Science: On the Pathway to the SKA. 25-27 May, 7

de Vaucouleurs, G., de Vaucouleurs, A., Corwin, Herold G., J., et al. 1991, Third Reference Catalogue of Bright Galaxies

Di Teodoro, E. M., \& Fraternali, F. 2014, A\&A, 567, A68

Dobbs, C. L., Theis, C., Pringle, J. E., \& Bate, M. R. 2010, MNRAS, 403, 625

D’Onghia, E., Madau, P., Vera-Ciro, C., Quillen, A., \& Hernquist, L. 2016, ApJ, 823, 4 
Fox, A. J., Lehner, N., Tumlinson, J., et al. 2013, ApJ, 778, 187

Fraternali, F., \& Tomassetti, M. 2012, MNRAS, 426, 2166

Genzel, R., Tacconi, L. J., Gracia-Carpio, J., et al. 2010, MNRAS, 407, 2091

Giovanelli, R., \& Haynes, M. P. 1988, Extragalactic neutral hydrogen, ed. K. I. Kellermann \& G. L. Verschuur, 522-562

Griffiths, D. J. 1982, American Journal of Physics, 50, 698. https://doi.org/10. $1119 / 1.12733$

Guo, Q., White, S., Boylan-Kolchin, M., et al. 2011, MNRAS, 413, 101

Haynes, M. P., Giovanelli, R., Martin, A. M., et al. 2011, AJ, 142, 170

Heald, G., Józsa, G., Serra, P., et al. 2011, A\&A, 526, A118

Heald, G., de Blok, W. J. G., Lucero, D., et al. 2016, MNRAS, 462, 1238

Hirschmann, M., Naab, T., Somerville, R. S., Burkert, A., \& Oser, L. 2012, MNRAS, 419,3200

Ianjamasimanana, R., Walter, F., de Blok, W. J. G., Heald, G. H., \& Brinks, E. 2018, AJ, 155, 233

Joung, M. R., Putman, M. E., Bryan, G. L., Fernández, X., \& Peek, J. E. G. 2012, ApJ, 759, 137

Kannappan, S. J., Stark, D. V., Eckert, K. D., et al. 2013, ApJ, 777, 42 
Katz, N., Keres, D., Dave, R., \& Weinberg, D. H. 2003, in Astrophysics and Space Science Library, Vol. 281, The IGM/Galaxy Connection. The Distribution of Baryons at $\mathrm{z}=0$, ed. J. L. Rosenberg \& M. E. Putman, 185

Kaviraj, S. 2014, MNRAS, 440, 2944

Kennicutt, R. C., J. 1983, ApJ, 272, 54

Kennicutt, R. C., \& Evans, N. J. 2012, ARA\&A, 50, 531

Kereš, D., Katz, N., Weinberg, D. H., \& Davé, R. 2005, MNRAS, 363, 2

Kim, S. Y., Peter, A. H. G., \& Hargis, J. R. 2017, arXiv e-prints, arXiv:1711.06267

Kleiner, D., Pimbblet, K. A., Jones, D. H., Koribalski, B. S., \& Serra, P. 2017, MNRAS, 466, 4692

Klypin, A., Kravtsov, A. V., Valenzuela, O., \& Prada, F. 1999, ApJ, 522, 82

Koribalski, B. S., Staveley-Smith, L., Kilborn, V. A., et al. 2004, AJ, 128, 16

Kourkchi, E., \& Tully, R. B. 2017, ApJ, 843, 16

Krug, H. B., Rupke, D. S. N., \& Veilleux, S. 2010, ApJ, 708, 1145

Larson, R. B. 1972a, Nature, 236, 21

—. 1972b, Nature Physical Science, 236, 7

Larson, R. B., \& Tinsley, B. M. 1978, ApJ, 219, 46

Lasker, B. M., Sturch, C. R., McLean, B. J., et al. 1990, AJ, 99, 2019 
Lauberts, A. 1982, ESO/Uppsala survey of the $\mathrm{ESO}(\mathrm{B})$ atlas

Lehner, N. 2017, in Astrophysics and Space Science Library, Vol. 430, Gas Accretion onto Galaxies, ed. A. Fox \& R. Davé, 117

Lehner, N., Howk, J. C., Tripp, T. M., et al. 2013, ApJ, 770, 138

Leitner, S. N., \& Kravtsov, A. V. 2011, ApJ, 734, 48

Leroy, A. K., Walter, F., Brinks, E., et al. 2008, AJ, 136, 2782

Leroy, A. K., Walter, F., Sandstrom, K., et al. 2013, AJ, 146, 19

Lotz, J. M., Jonsson, P., Cox, T. J., \& Primack, J. R. 2010, MNRAS, 404, 590

Madau, P., \& Dickinson, M. 2014, ARA\&A, 52, 415

Maloney, P. 1993, ApJ, 414, 41

Masters, K. L., Springob, C. M., \& Huchra, J. P. 2014, AJ, 147, 124

Meurer, G. R., Hanish, D. J., Ferguson, H. C., et al. 2006, ApJS, 165, 307

Meyer, M. J., Zwaan, M. A., Webster, R. L., et al. 2004, MNRAS, 350, 1195

Mihos, J. C., Keating, K. M., Holley-Bockelmann, K., Pisano, D. J., \& Kassim, N. E. 2012, ApJ, 761, 186

Moore, B., Ghigna, S., Governato, F., et al. 1999, ApJ, 524, L19

Moster, B. P., Somerville, R. S., Maulbetsch, C., et al. 2010, ApJ, 710, 903

Navarro, J. F., Frenk, C. S., \& White, S. D. M. 1996, ApJ, 462, 563 
Noterdaeme, P., Petitjean, P., Carithers, W. C., et al. 2012, A\&A, 547, L1

Oh, S. H., Kim, W.-T., \& Lee, H. M. 2015, ApJ, 807, 73

Oosterloo, T., Fraternali, F., \& Sancisi, R. 2007, AJ, 134, 1019

Pauli, W. 1940, Physical Review, 58, 716. https://doi:10.1103/PhysRev.58.716.

Peebles, P. J. E. 1982, ApJL, 263, L1

Pingel, N. M., Pisano, D. J., Heald, G., et al. 2018, ApJ, 865, 36

Pisano, D. J., \& Wilcots, E. M. 2000, MNRAS, 319, 821

Popping, A., Davé, R., Braun, R., \& Oppenheimer, B. D. 2009, A\&A, 504, 15

Popping, A., Meyer, M., Staveley-Smith, L., et al. 2015, in Advancing Astrophysics with the Square Kilometre Array (AASKA14), 132

Price, D. C., Staveley-Smith, L., Bailes, M., et al. 2016, Journal of Astronomical Instrumentation, 5, 1641007

Prochaska, J. X. 1999, ApJL, 511, L71

Putman, M. E., de Heij, V., Staveley-Smith, L., et al. 2002, AJ, 123, 873

Qu, Z., Bregman, J. N., \& Hodges-Kluck, E. J. 2019, ApJ, 876, 101

Rhee, J., Lah, P., Briggs, F. H., et al. 2018, MNRAS, 473, 1879

Ribaudo, J., Lehner, N., Howk, J. C., et al. 2011, ApJ, 743, 207 
Roychowdhury, S., Chengalur, J. N., Kaisin, S. S., \& Karachentsev, I. D. 2014, Monthly Notices of the Royal Astronomical Society, 445, 1392

Rubin, K. H. R., Prochaska, J. X., Koo, D. C., et al. 2014, ApJ, 794, 156

Sales, L. V., Wang, W., White, S. D. M., \& Navarro, J. F. 2013, MNRAS, 428, 573

Salo, H., \& Laurikainen, E. 1993, ApJ, 410, 586

Sato, T., Martin, C. L., Noeske, K. G., Koo, D. C., \& Lotz, J. M. 2009, ApJ, 696, 214

Schaye, J., Crain, R. A., Bower, R. G., et al. 2015, MNRAS, 446, 521

Schechter, P. 1976, ApJ, 203, 297

Schiminovich, D., Catinella, B., Kauffmann, G., et al. 2010, MNRAS, 408, 919

Scudder, J. M., Ellison, S. L., Torrey, P., Patton, D. R., \& Mendel, J. T. 2012, MNRAS, 426, 549

Semczuk, M., Łokas, E. L., Salomon, J.-B., Athanassoula, E., \& D'Onghia, E. 2018, ApJ, 864, 34

Skrutskie, M. F., Cutri, R. M., Stiening, R., et al. 2006, AJ, 131, 1163

Sorgho, A., Foster, T., Carignan, C., \& Chemin, L. 2019a, MNRAS, 486, 504

Sorgho, A., Carignan, C., Pisano, D. J., et al. 2019b, MNRAS, 482, 1248

-. 2019c, MNRAS, 482, 1248 
Springel, V., White, S. D. M., Jenkins, A., et al. 2005, Nature, 435, 629

Staveley-Smith, L., Wilson, W. E., Bird, T. S., et al. 1996, PASA, 13, 243

Stevens, A. R. H., Diemer, B., Lagos, C. d. P., et al. 2019, MNRAS, 483, 5334

Stocke, J. T., Keeney, B. A., \& Danforth, C. W. 2010, PASA, 27, 256

Tacconi, L. J., Neri, R., Genzel, R., et al. 2013, ApJ, 768, 74

Tinsley, B. M. 1980, Fund. Cosmic Phys., 5, 287

Tully, R. B. 1988, Nearby galaxies catalog

Tumlinson, J., Thom, C., Werk, J. K., et al. 2011, Science, 334, 948

—. 2013, ApJ, 777, 59

Verheijen, M. A. W., \& Sancisi, R. 2001, A\&A, 370, 765

Walter, F., Brinks, E., de Blok, W. J. G., et al. 2008, AJ, 136, 2563

Werk, J. K., Prochaska, J. X., Tumlinson, J., et al. 2014, ApJ, 792, 8

Westmeier, T., Obreschkow, D., Calabretta, M., et al. 2017, MNRAS, 472, 4832

White, M. 2001, A\&A, 367, 27

White, S. D. M., \& Frenk, C. S. 1991, ApJ, 379, 52

Winkel, B., Kerp, J., Flöer, L., et al. 2016, A\&A, 585, A41

Wolfe, S. A., Lockman, F. J., \& Pisano, D. J. 2016, ApJ, 816, 81 
Wolfe, S. A., Pisano, D. J., Lockman, F. J., McGaugh, S. S., \& Shaya, E. J. 2013, Nature, 497, 224 